\title{
Thermal Characterization of Honeycomb Core Sandwich Structures
}

\author{
By
}

\author{
David C. Copenhaver
}

\begin{abstract}
Thesis submitted to the Faculty of the
Virginia Polytechnic Institute and State University

in partial fulfillment of the requirements for the degree of
\end{abstract}

MASTER OF SCIENCE

in

Mechanical Engineering

APPROVED:
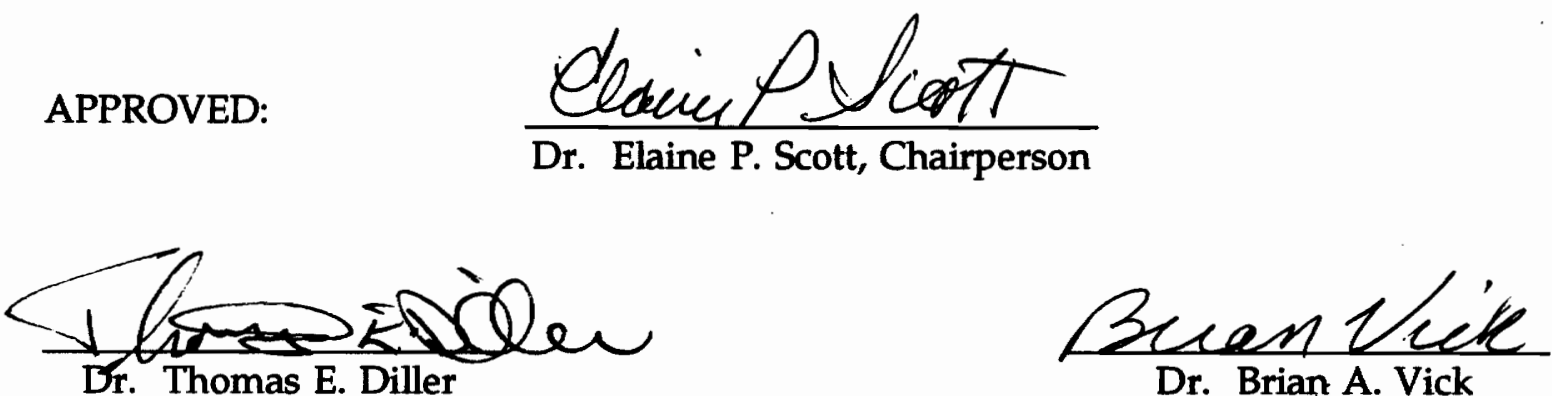

March 21, 1996

Blacksburg, Virginia

Key Words: Parameter Estimation, Heat Transfer, Sandwich Structures 


$$
\begin{aligned}
& L D \\
& 5655 \\
& V 855 \\
& 1996 \\
& C 674 \\
& C .2
\end{aligned}
$$




\section{Acknowledgements}

I would like to thank the following people for the help they have given me in performing this research:

Dr. Elaine P. Scott, my and advisor and mentor, for all the help and guidance she has given me. Thank you for giving me this project to work on, for giving me freedom in doing it, and for advice when I needed it. It has been a privilege to work with you and to know you. 1507).

NASA Langley Research Center, for funding this research (Grant No. NAG-1-

Dr. Stephen J. Scotti, for all his assistance this summer, and for solving my EAL problems.

Max Blosser, Kamran Daryabeigi, Tom Sutter, and Joe O'Donnell at NASA for the help in getting my experiments to work.

Dr. Thomas Diller and Dr. Brian Vick, for serving on my advisory committee.

Greg, Joe, Pierre, Jim, Sandrine, and the rest of the people in the lab for answering my questions.

Jamie Hale and Tommy Bunch, my roommates, for being good friends

My parents, for their love and guidance, for supporting my decisions, and for putting me through college. 


\title{
Thermal Characterization of Honeycomb Core Sandwich Structures
}

\author{
by \\ David C. Copenhaver \\ Committee Chairperson: Dr. Elaine P. Scott \\ Mechanical Engineering
}

(ABSTRACT)

Honeycomb core sandwich structures are an integral part of many of today's aerospace structures. When subjected to high speed flight, thermal loading can induce significant stresses. The need for thermal properties to perform thermal stress analyses in these structures is the motivation behind this research. Thermal property estimation approach used here involves the minimization of a least squares function containing both measured and calculated values. In addition, an applied heat flux is necessary at one boundary for the simultaneous estimation of thermal properties. The specific objectives are to develop a thermal model to describe honeycomb core sandwich structures, optimize experimental designs for use in parameter estimation, develop a finite element based parameter estimation algorithm, and estimate the pertinent thermal properties of the structure.

A combined conductive/radiative heat transfer model was used for the analysis of the structure. Due to the composition of the structure, it was determined that a one dimensional model would be sufficient. This model was used in both parameter estimation and experimental design.

Experimental design involves finding input variables for an experiment such that the response of the system contains the highest possible amount of information on the parameters of interest which characterize the response. In this study, the design was performed by using a combination of two methods. The first involved maximizing the 
temperature derivatives with respect to unknown thermal properties. The second involved a scaled confidence interval approach. The experimental parameters optimized were heating time and total experiment time.

A finite element program was used to perform transient temperature calculations because of the flexibility it has to analyze complex structures. Parameters estimated in this study exhibited a great deal of correlation, or interaction. This showed the need for a constrained parameter estimation algorithm. A penalty function method was developed for this purpose.

The last part of this study involved the actual estimation of thermal properties. An experimental apparatus was designed and built to record the transient temperature response of the test sample. A four sheet SPF/DB sandwich was used as the test sample. Thermal properties were estimated using four combinations of sensors and boundary conditions.

It was found that in one case parameters could be simultaneously estimated despite the presence of correlation. These estimated parameters were shown to produce reasonably small errors when used in transient temperature calculations. It was also shown that large temperature gradients produce estimates with smaller confidence intervals. The importance of maintaining accurately known boundary conditions was also demonstrated. 


\section{Table of Contents}

List of Tables. ........................... vii

List of Figures. $\ldots \ldots \ldots \ldots \ldots \ldots \ldots \ldots \ldots \ldots \ldots \ldots \ldots \ldots \ldots \ldots$

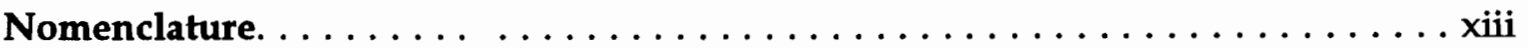

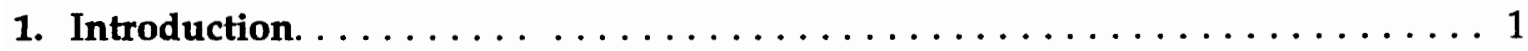

1.1 Goals and Objectives. . . . . . . . . . . . . . . . . . 2

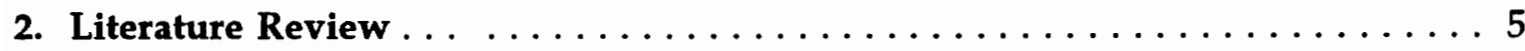

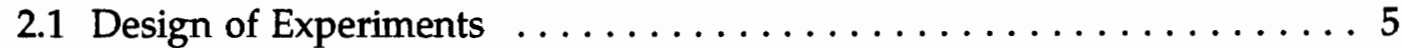

2.2 Estimation Procedures. . . . . . . . . . . . . . . . . 7

2.3 Property Estimation in Sandwich Structures. . . . . . . . . . . . 11

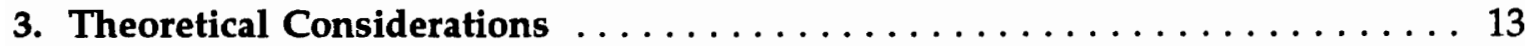

3.1 Heat Transfer Model. . . . . . . . . . . . . . . . . . . . . . . . . 14

3.2 Minimization Procedure ....................... 21

3.2.1 Modified Box-Kanemasu. . . . . . . . . . . . . . . 22

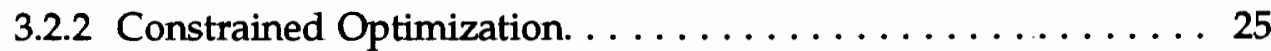

3.3 Confidence Regions and Intervals. . . . . . . . . . . . 31

3.4 Design of Experiments. . . . . . . . . . . . . . . 32

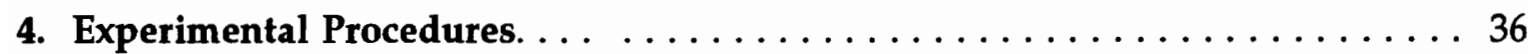

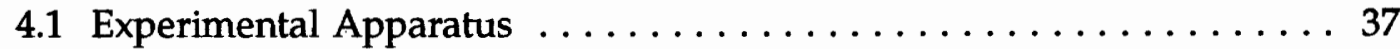

4.1 .1 Test Samples . . . . . . . . . . . . . . . . 37

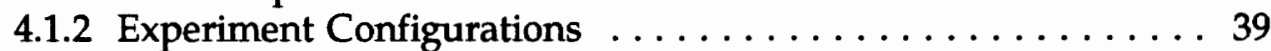

4.1 .3 Experimental Setup . . . . . . . . . . . . . . 41

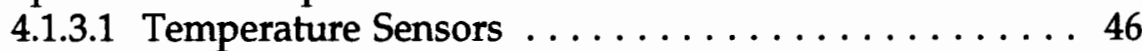

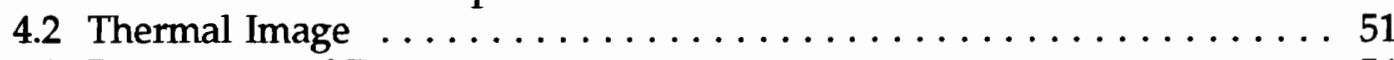

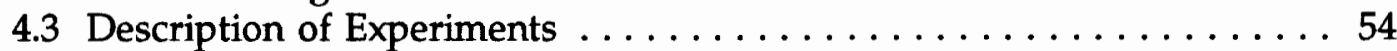

4.4 Experimental Data . . . . . . . . . . . . . . . . . 56

5. Implementation of the Finite Element Program $\ldots \ldots \ldots \ldots \ldots$

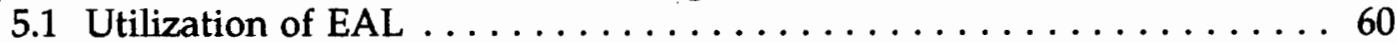

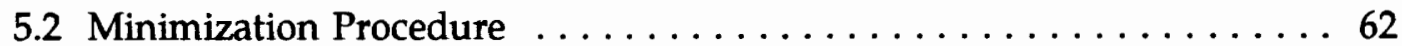

5.2 .1 Experimental Data . . . . . . . . . . . . . . 65

5.2 .2 Minimization Algorithm .............. 65

5.3 Experimental Design . . . . . . . . . . . . . . . . 68

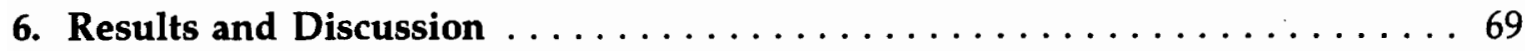

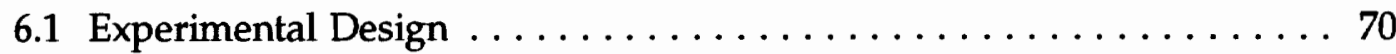

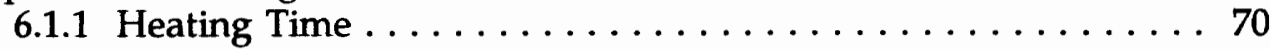

6.1.2 Total Experiment Time . . . . . . . . . . . . 79 
6.1 .3 Other Factors $\ldots \ldots \ldots \ldots \ldots \ldots \ldots \ldots \ldots \ldots \ldots \ldots$

6.2 Sensitivity Coefficients $\ldots \ldots \ldots \ldots \ldots \ldots \ldots \ldots \ldots \ldots \ldots \ldots$

6.3 Estimation Results .......................... 91

6.3.1 Specified Temperature Boundary Results ............ 91

6.3.2 Estimation Results for the Insulated Boundary With Two Sensors ........................ 95

6.3.3 Estimation Results for the Insulated Boundary With One Sensor ......................... 98

6.3.4 Estimation Results for Both Boundary Conditions Combined 101

6.3.5 Comparison of Estimated Property Values ............ 104

6.4 Statistical Interpretation of Results . .................. 105

6.5 High Temperature Numerical Experiments . . . . . . . . . . . 108

6.6 Confidence Regions ........................ 110

6.7 Heat Transfer Modes in the Structure $\ldots \ldots \ldots \ldots \ldots \ldots \ldots \ldots . \ldots \ldots$

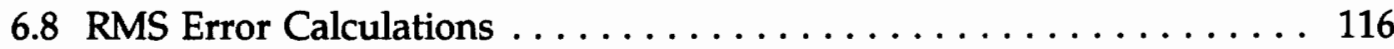

7. Conclusions and Summary $\ldots \ldots \ldots \ldots \ldots \ldots \ldots \ldots \ldots \ldots \ldots \ldots \ldots$

7.1 Heat Transfer Model . . . . . . . . . . . . . . . . . . . . 119

7.2 Experimental Design ........................ 120

7.3 Parameter Estimation Algorithm ..................... 120

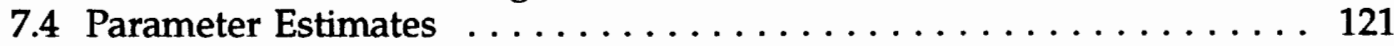

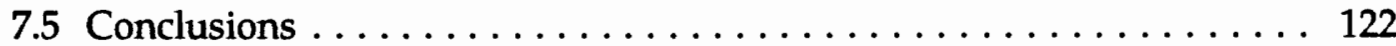

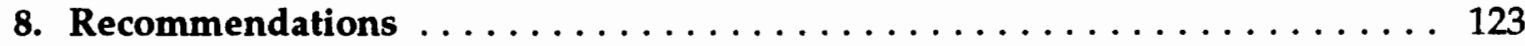

Bibliography. ............................... 126

Appendix A. Scaled Confidence Intervals and Eigenvalues $\ldots \ldots \ldots \ldots \ldots 130$

Appendix B. Parameter Estimation Program: HCEST ............. 140

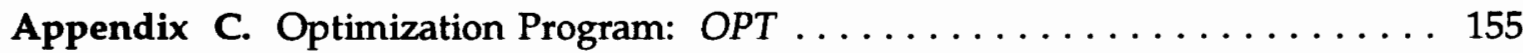

Appendix D. Radiation View Factor Code $\ldots \ldots \ldots \ldots \ldots \ldots \ldots \ldots \ldots . \ldots . \ldots$

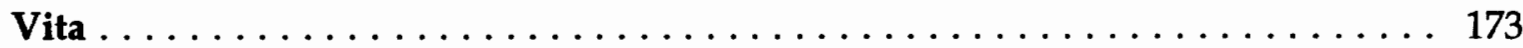




\section{List of Tables}

Table 3.1 View Factors, $\mathrm{F}_{\mathrm{ij}}$, Between Elements in the Unit Cell, where $i$ is the emitting element and $j$ is the incident element $\ldots \ldots \ldots 19$

Table $5.1 \quad$ Sample EAL Runstream $\ldots \ldots \ldots \ldots \ldots \ldots \ldots \ldots \ldots \ldots \ldots \ldots$

Table 6.1 Optimum Heating Times for the Specified Temperature Boundary Case Using the $\left|\boldsymbol{X}^{\mathrm{T}} \boldsymbol{X}\right|$ Criteria . . . . . . . . . . . . 71

Table 6.2 Recommended Heating Times for the Specified Temperature Boundary Case ....................... 74

Table 6.3 Recommended Heating Times for the Insulated Boundary Case . . . 79

Table 6.4 Recommended Total Experiment Times for the Specified Temperature Boundary Case ...................... 81

Table 6.5 Recommended Total Experiment Times for the Insulated Boundary Case ........................ 86

Table 6.6 Mean Estimate Values for all Initial Temperatures for the Specified Temperature Boundary Case . . . . . . . . . . . . . . . 95

Table 6.7 Mean Estimate Values for all Initial Temperatures for the Insulated Boundary Case Using Information From Two Sensors . . . . . . . . 98

Table 6.8 Mean Estimate Values at all Initial Temperatures for the Insulated Boundary Case Using Information From One Sensor . . . . . . . . 101

Table 6.9 Mean Estimate Values at all Initial Temperatures for Estimation with Both Boundary Conditions . . . . . . . . . . . . . . . . . . 104

Table $6.10 \quad$ Analysis of Variance for Estimated Parameters . . . . . . . . . . 107

Table 6.11 Numerical Experiment at $695 \mathrm{~K}$ for the Specified Temperature Boundary ......................... 109

Table 6.12 Average RMS Error Calculations for Specified Temperature and Insulated (Two Sensor) Boundaries . . . . . . . . . . . 117

Table 6.13 RMS Temperature Errors Corresponding to Parameter Confidence Intervals for the Specified Temperature Boundary Condition . . . . 118

Table B.1 Program HCEST $\ldots \ldots \ldots \ldots \ldots \ldots \ldots \ldots \ldots \ldots \ldots \ldots \ldots \ldots \ldots$ 


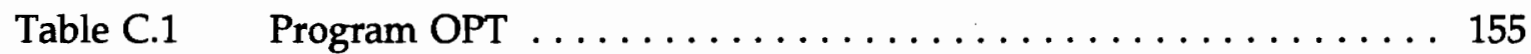

Table D.1 Radiation View Factor Code $\ldots \ldots \ldots \ldots \ldots \ldots \ldots$ 


\section{List of Figures}

Figure 2.1 Examples of Nonlinear Optimization/Estimation Methods . . . . . . 8

Figure $3.1 \quad$ Heat Transfer Model Boundary Conditions $\ldots \ldots \ldots \ldots \ldots$

Figure 3.2a Detail of a Single Core Element $\ldots \ldots \ldots \ldots \ldots \ldots \ldots \ldots$

Figure 3.2b Diagram of Computational Model Enclosure Elements . . . . . . . 17

Figure $3.3 \quad$ Exterior Penalty Function Algorithm $\ldots \ldots \ldots \ldots \ldots \ldots$

Figure $3.4 \quad$ Unconstrained Minimization Algorithm . . . . . . . . . . . 29

Figure 4.1 Honeycomb Core SPF/DB Phases of Development . . . . . . . 38

Figure 4.2 Core and Face Sheet Dimensions of a Typical Sample . . . . . . 40

Figure 4.3a Constant Temperature Boundary Experiment $\ldots \ldots \ldots \ldots \ldots$

Figure $4.3 b \quad$ Insulated Boundary Experiment $\ldots \ldots \ldots \ldots \ldots \ldots$

Figure $4.4 \quad$ Overall Setup $\ldots \ldots \ldots \ldots \ldots \ldots \ldots \ldots \ldots \ldots \ldots \ldots \ldots$

Figure $4.5 \quad$ RTD Calibration Curve $\ldots \ldots \ldots \ldots \ldots \ldots \ldots \ldots \ldots \ldots$

Figure $4.6 \quad$ RTD Bridge Circuit $\ldots \ldots \ldots \ldots \ldots \ldots \ldots \ldots \ldots \ldots \ldots$

Figure $4.7 \quad$ Thermal Image of Honeycomb Structure $\ldots \ldots \ldots \ldots \ldots \ldots$

Figure 4.8 Diagram of Camera View of Structure in Thermal Image . . . . . 53

Figure 4.9 Constant Temperature Boundary Data $\ldots \ldots \ldots \ldots \ldots$

Figure $4.10 \quad$ Insulated Boundary Data $\ldots \ldots \ldots \ldots \ldots \ldots \ldots \ldots$

Figure $5.1 \quad$ Finite Element Formulation Using EAL $\ldots \ldots \ldots \ldots \ldots$

Figure $5.2 \quad$ Estimation Program Flow Chart $\ldots \ldots \ldots \ldots \ldots \ldots \ldots \ldots \ldots$

Figure 6.1 Determinant of $\left(\boldsymbol{X}^{\mathbf{T}} \boldsymbol{X}\right)$ for the Specified Temperature Boundary Case, Used to Determine Heating Time . . . . . . . . . . . 72

Figure 6.2 Scaled Confidence Intervals at $295 \mathrm{~K}$ for the Specified Temperature Boundary Case, Used to Determine Heating Time . . . . . . . . 73 
Figure 6.3 Scaled Confidence Intervals at $495 \mathrm{~K}$ for the Specified Temperature Boundary Case, Used to Determine Heating Time .......... 73

Figure 6.4 Determinant Eigenvalues at $295 \mathrm{~K}$ for the Specified Temperature Boundary Case, Used to Determine Heating Time ......... 75

Figure 6.5 Determinant Eigenvalues at $495 \mathrm{~K}$ for the Specified Temperature Boundary Case, Used to Determine Heating Time . . . . . . . 75

Figure 6.6 Determinant of $\left(\boldsymbol{X}^{T} \boldsymbol{X}\right)$ for the Insulated Boundary Case, Used to Determine Heating Time $. . \ldots \ldots \ldots \ldots \ldots \ldots . . \ldots \ldots$

Figure 6.7 Scaled Confidence Intervals at $295 \mathrm{~K}$ for the Insulated Boundary Case, Used to Determine Heating Time $\ldots \ldots \ldots \ldots \ldots \ldots .78$

Figure 6.8 Scaled Confidence Intervals at $495 \mathrm{~K}$ for the Insulated Boundary Case, Used to Determine Heating Time $\ldots \ldots \ldots \ldots \ldots \ldots .78$

Figure 6.9 Determinant Eigenvalues at $295 \mathrm{~K}$ for the Insulated Boundary Case, Used to Determine Heating Time $\ldots \ldots \ldots \ldots \ldots \ldots \ldots .80$

Figure 6.10 Determinant Eigenvalues at $495 \mathrm{~K}$ for the Insulated Boundary Case, Used to Determine Heating Time $\ldots \ldots \ldots \ldots \ldots \ldots \ldots .80$

Figure 6.11 Determinant of $\left(\mathrm{X}^{\mathrm{T}} \mathrm{X}\right)$ for the Specified Temperature Boundary Case, Used to Determine Total Experiment Time ............. 82

Figure 6.12 Scaled Confidence Intervals at $295 \mathrm{~K}$ for the Specified Temperature Boundary Case, Used to Determine Total Experiment Time .... . 83

Figure 6.13 Scaled Confidence Intervals at $495 \mathrm{~K}$ for the Specified Temperature Boundary Case, Used to Determine Total Experiment Time ..... 83

Figure 6.14 Determinant of $\left(\mathrm{X}^{T} \mathrm{X}\right)$ for the Insulated Boundary Case, Used to Determine Total Experiment Time $\ldots \ldots \ldots \ldots \ldots \ldots \ldots \ldots 84$

Figure 6.15 Scaled Confidence Intervals at $295 \mathrm{~K}$ Used to Determine Total Experiment Time for the Insulated Boundary Case ........ 85

Figure 6.16 Scaled Confidence Intervals at $495 \mathrm{~K}$ Used to Determine Total Experiment Time for the Insulated Boundary Case ......... 85

Figure 6.17 Dimensionless Sensitivity Coefficients for the Specified Temperature Boundary Case at $295 \mathrm{~K}$ at the Heated Surface . . . . . . . . 88

Figure 6.18 Dimensionless Sensitivity Coefficients for the Specified Temperature Boundary Case at $495 \mathrm{~K}$ at the Heated Surface ........... 88 
Figure 6.19 Dimensionless Sensitivity Coefficients for the Insulated Boundary Case at $295 \mathrm{~K}$ at Both Surfaces . . . . . . . . . . . . . . . . . . 90

Figure 6.20 Dimensionless Sensitivity Coefficients for the Insulated Boundary Case at $495 \mathrm{~K}$ at Both Surfaces . . . . . . . . . . . . . . 90

Figure 6.21 Diagram of Information Used in the Four Estimation Cases . . . . 92

Figure 6.22 Emissivity Estimates for the Specified Temperature Boundary Condition

Figure 6.23 Conduction Area Estimates for the Specified Temperature Boundary 93

Figure 6.24 Volumetric Heat Capacity Estimates for the Specified Temperature Boundary ......................... 94

Figure 6.25 Emissivity Estimates for the Insulated Boundary Condition With Two Sensors . . . . . . . . . . . . . . . . . . 96

Figure 6.26 Conduction Area Estimates for the Insulated Boundary Condition With Two Sensors . . . . . . . . . . . . . . . . . 96

Figure 6.27 Volumetric Heat Capacity Estimates for the Insulated Boundary Condition With Two Sensors ... . . . . . . . . . . . . . . . . 97

Figure 6.28 Emissivity Estimates for the Insulated Boundary Condition With One Sensor . . . . . . . . . . . . . . . . . . . . . . . 99

Figure 6.29 Conduction Area Estimates for the Insulated Boundary Condition With One Sensor . . . . . . . . . . . . . . . . . . . . . . . . 99

Figure 6.30 Volumetric Heat Capacity Estimates for the Insulated Boundary Condition With Two Sensors . . . . . . . . . . . . . . . . . . 100

Figure 6.31 Emissivity Estimates for Both Boundary Conditions . . . . . . . 102

Figure 6.32 Conduction Area Estimates for Both Boundary Conditions . . . . . 102

Figure 6.33 Volumetric Heat Capacity Estimates for Both Boundary Conditions 103

Figure 6.34 Confidence Region for the Insulated Boundary Case With Two Sensors ............................. 111

Figure 6.35 Confidence Region for the Specified Temperature Boundary Case . 112

Figure 6.36 Heat Flux Components for the Specified Temperature Boundary Case .............................. 114 
Figure 6.37 Heat Flux Components for the Insulated Boundary Case $\ldots \ldots \ldots 115$ 


\section{Nomenclature}
A Area
b Estimated parameter vector
C Capacitance matrix
$\mathrm{C}_{\mathrm{p}} \quad$ Specific Heat $(\mathrm{J} / \mathrm{kg} \mathrm{K})$
D Variable metric method update matrix
$\mathrm{e}_{\mathrm{jk}} \quad$ Eigenvector component
$E_{i} \quad$ Eigenvalue
$F_{1-\alpha} \quad F-$ distribution
$F_{\mathrm{ij}} \quad$ View factor
F Boundary condition matrix in EAL, objective function
G Scalar used in Box-Kanemasu method
h Scalar interpolation function used in Box-Kanemasu method
H Inverse of Hessian matrix
I Electric current (A)
I Identity matrix
k Thermal conductivity
K Stiffness matrix
$\mathbf{K}_{\mathbf{k}} \quad$ Conduction load vector
$\mathbf{K}_{\mathbf{r}} \quad$ Effective radiation load vector
L Lagrangian
M Number of temperature sensors
N Number of temperature measurements 
p Number of parameters estimated

p Vector used in variable metric method

P Penalty function

$\mathbf{P} \quad$ Vector equal to $\left(\mathbf{X}^{\mathrm{T}} \mathbf{X}\right)^{-1}$

q" Heat flux $\left(W / m^{2}\right)$

$\mathrm{q}_{\text {cond }}$ Heat flux due to conduction $\left(\mathrm{W} / \mathrm{m}^{2}\right)$

$\mathrm{q}_{\text {rad }}$ Heat flux due to radiation $\left(\mathrm{W} / \mathrm{m}^{2}\right)$

r radius or scalar

$\mathbf{r}_{\mathrm{ij}} \quad$ Correlation matrix

$\mathbf{r}_{\mathrm{p}} \quad$ Penalty parameter

R Resistance ( $\Omega$ )

s Estimate of standard deviation

S Sum of squares

$\mathrm{S}_{0} \quad$ Sum of squares value at zero

$S_{\alpha} \quad$ Sum of squares value at $\alpha$

S Sum of squares function

$\mathbf{S}^{(\mathbf{k})} \quad$ Search Direction

t Time (sec)

$t_{1-\alpha} \quad$ Student's $t$ - distribution

T Temperature (K)

T Calculated temperature vector

V Voltage (volts)

$X \quad$ Used in view factor calculation

X Sensitivity coefficient matrix 
y Vector used in variable metric method

Y Used in view factor calculation

Y $\quad$ Measured temperature vector

\section{Greek}

$\alpha \quad$ Scalar used in Box-Kanemasu method

B Estimated parameter vector, weight factor

$\delta \quad$ Perturbation

$\varepsilon \quad$ emissivity

$\lambda \quad$ Lagrange multiplier

$\lambda_{\mathrm{i}} \quad$ Eigenvalue

$\eta \quad$ Calculated temperature vector

$\theta \quad$ Variable metric method scaling factor

$\rho \quad$ Density $\left(\mathrm{kg} / \mathrm{m}^{3}\right)$

$\Phi$ Effective objective function

$\sigma \quad$ Scalar used in variable metric method

$\tau \quad$ Vector used in variable metric method

$\chi \quad$ General vector of independent variables

$\nabla \quad$ Gradient operator

$\Delta_{\mathbf{b}} \mathbf{b} \quad$ Vector used in Box-Kanemasu method

\section{Superscripts}

k Iteration number

T Transpose

$\Delta t \quad$ Time step 


\section{CHAPTER 1}

\section{Introduction}

The high strength-to-weight ratio of honeycomb core structures makes them ideal for use in aircraft and aerospace vehicles. The properties of these structures must be known for design purposes. Convective heating in high speed flight can induce large temperature gradients which induce thermal stresses, as part of the mechanical load on the structures. Calculation of thermal stresses requires knowledge of temperature distributions within a material, which requires knowledge of the material's thermal properties.

The motivation for this research is the need to predict thermal properties in honeycomb core sandwich structures so that the information can later be used in a thermal stress analysis. Since these structures are complex and unique, it was also necessary to find a way to model the heat transfer through them. A method of experimental design was also developed to assist in the thermal property estimation.

A honeycomb core sandwich structure consists of two thin face sheets which are held apart by a core. The core has a matrix or grid design. Its purpose is to resist compressive forces and prevent the two face sheets from moving toward each other under load. This gives the structure a high moment of inertia to resist bending stress, 
but with a relatively low weight. The face sheets and core can made of many materials, including graphite composites and metals. The type investigated in this study were constructed mainly of a titanium alloy.

This work is a continuation of previous analyses performed by Moncman (1994) and Hanak (1995). Moncman developed experimental designs for the estimation of thermal properties of carbon composites subjected to one and two dimensional heat transfer. Hanak focused on experimental verification of these designs. This investigation is part of a much larger project, the goal of which is to develop methodologies for the estimation of thermal properties and interface conditions in large, complex aerospace structures.

\subsection{Goals and Objectives}

The purpose of this study was to develop a method for the estimation of thermal properties in honeycomb core sandwich structures. The material being examined was a four sheet, super-plastically formed/double bonded sandwich panel, composed of a Ti$6 \mathrm{Al}-4 \mathrm{~V}$ alloy interior with aluminum-boron composite face sheets. In order to achieve the goals of this research, the specific objectives were to:

1) develop a conductive/radiative finite element model which describes heat transfer through the thickness of the structure,

2) find optimal or near-optimal experimental design parameters for the collection of temperature response data necessary for parameter estimation, 
3) develop a parameter estimation algorithm which is capable of being used within the framework of a finite element code, and

4) estimate the unknown thermal properties in the heat transfer model.

The first objective deals with finding an appropriate heat transfer model for the structure. Since conduction and radiation heat transfer both exist within the structure, a combined model was developed. A finite element routine was used for temperature calculations due to complexities caused by nonlinear radiation terms. A one dimensional model was found to be sufficient to model the heat transfer within the structure.

The goal of most experiments is to produce results which have the highest possible accuracy and lowest variability. Some experimental design is usually necessary to accomplish this. In the case of parameter estimation, a least squares comparison is made between calculated data and measured data from an actual experiment. Physical experiments should be run which maximize the sensitivity of measured temperature data to changes in unknown thermal properties, while yielding the smallest confidence intervals on these unknown properties.

The experimental design was based on a standard approach dealing with sensitivity coefficients, which related changes in the unknown thermal properties to changes in temperature. A modification of this sensitivity coefficient approach was investigated which used the lengths of scaled confidence intervals. The experimental parameters which were varied include the heating time and experiment duration. An applied heat flux was necessary for one boundary. Two other conditions were examined at the other boundary. 
A parameter estimation procedure was developed to estimate thermal properties in the sandwich structure. The Gauss linearization method was the first method that was tried. It uses a first order algorithm to minimize a least squares objective function containing both experimental and calculated temperatures. After it became apparent that the Gauss method would not converge, a penalty function method was developed. It minimized the same objective function as before.

A series of experiments were run to collect data necessary for the parameter estimation. These experiments were performed for a range of initial temperatures and for two boundary conditions. Three thermal properties were estimated in this study; however, the methods used could be theoretically expanded to estimate any number of properties. 


\section{CHAPTER 2}

\section{Literature Review}

Much work has been done in the area of parameter estimation and optimization. Many methods have been developed which are often specific to a certain field, with none being a standard. Thermal property estimation has been performed extensively on isotropic and composite materials. However, there are few cited cases of thermal property estimation dealing with sandwich structures. What little work that has been done in this area is mostly analytical in nature. This chapter gives a synopsis of parameter estimation and optimization techniques. It also describes sandwich structures and how parameter estimation and analysis has been performed on them.

\subsection{Design of Experiments}

The goal of any parameter estimation procedure is to produce estimates which have the greatest amount of accuracy. This can best be accomplished by careful experimental design. A carefully designed experiment is one in which there is minimum correlation between the estimated properties, as well as maximum sensitivity of the measured experimental variables to changes in the properties being estimated (Beck and 
Arnold, 1977). The design process involves the determination of optimal experimental variables through the use of a design criteria.

Many design criteria have been proposed. These criteria are often denoted by letters. Beck and Arnold (1977) list several design criteria based on the sensitivity of the estimated parameters to changes in the response variable. These are usually given in terms of the product of the sensitivity coefficients and their transpose, $\boldsymbol{X}^{T} \boldsymbol{X}$. They include the following: maximization of the determinant of $\boldsymbol{X}^{T} \boldsymbol{X}$ (D-optimal), maximization of the minimum eigenvalue of $\boldsymbol{X}^{T} \boldsymbol{X}$ (C-optimal), and maximization of the trace of $\boldsymbol{X}^{T} \boldsymbol{X}$ (Aoptimal).

The D-optimal criterion has been widely used and studied. It was used by Beck (1969) to optimize experimental parameters for the simultaneous estimation of thermal conductivity and specific heat. Taktak, et al. (1991) used the procedure to optimize heating time, sensor location, and number of sensors for the estimation of volumetric heat capacity and thermal conductivity in carbon-fiber/epoxy-matrix composite materials. Bayard et al. (1988) used the D-optimal criterion to optimize sensor location for identification experiments in large space structures. Glucose tests in humans were the focus of work by Cobelli and Ruggeri (1991) who sought to obtain accurate results from a minimum number of experiments. Moncman (1994) used the procedure to derive experimental designs for thermal property estimation in anisotropic composites. Her work was continued and partially verified by Hanak (1995).

There are variations of the D-optimal criteria which have been proposed. Prozonto and Walter (1989) defined the V-criterion, which is similar to the D-optimal but assumes that upper and lower bounds are known for noise associated with the measurements. Lohmann et al (1992) developed a related criterion of minimization of 
the maximum scaled length of the confidence intervals. A similar approach is used in this study.

\subsection{Estimation Procedures}

Numerous techniques have been developed for parameter estimation and optimization. Parameter estimation may be treated as a special case of optimization. Generally, optimization involves the use of an objective function which seeks to minimize the cost, weight, or size of a structure or process. The objective function in parameter estimation is a least squares function which compares measured and calculated parameters in a known model. While parameter estimation has generally been performed with only a few methods, it could conceivably use any optimization technique.

Optimization techniques can be divided into two classes as shown in Fig. 2.1. Unconstrained methods solve the optimization problem by finding the minimum of the objective function. This is accomplished by either a direct search or by using gradient information (descent methods). Unconstrained direct methods are zero order and may involve a random search. Descent methods are usually first or second order. Algorithms which permit the use of constraints may be direct or indirect. Direct constrained methods handle constraints explicitly, usually by a regularization technique which tests for feasibility at each iteration. Indirect methods effectively incorporate the constraints into the objective function and reduce the problem into one which is then solved by an unconstrained method.

There are three main types of parameter estimators. These include ordinary least squares (OLS), maximum likelihood (ML), and maximum a posteriori (MAP). Ordinary 


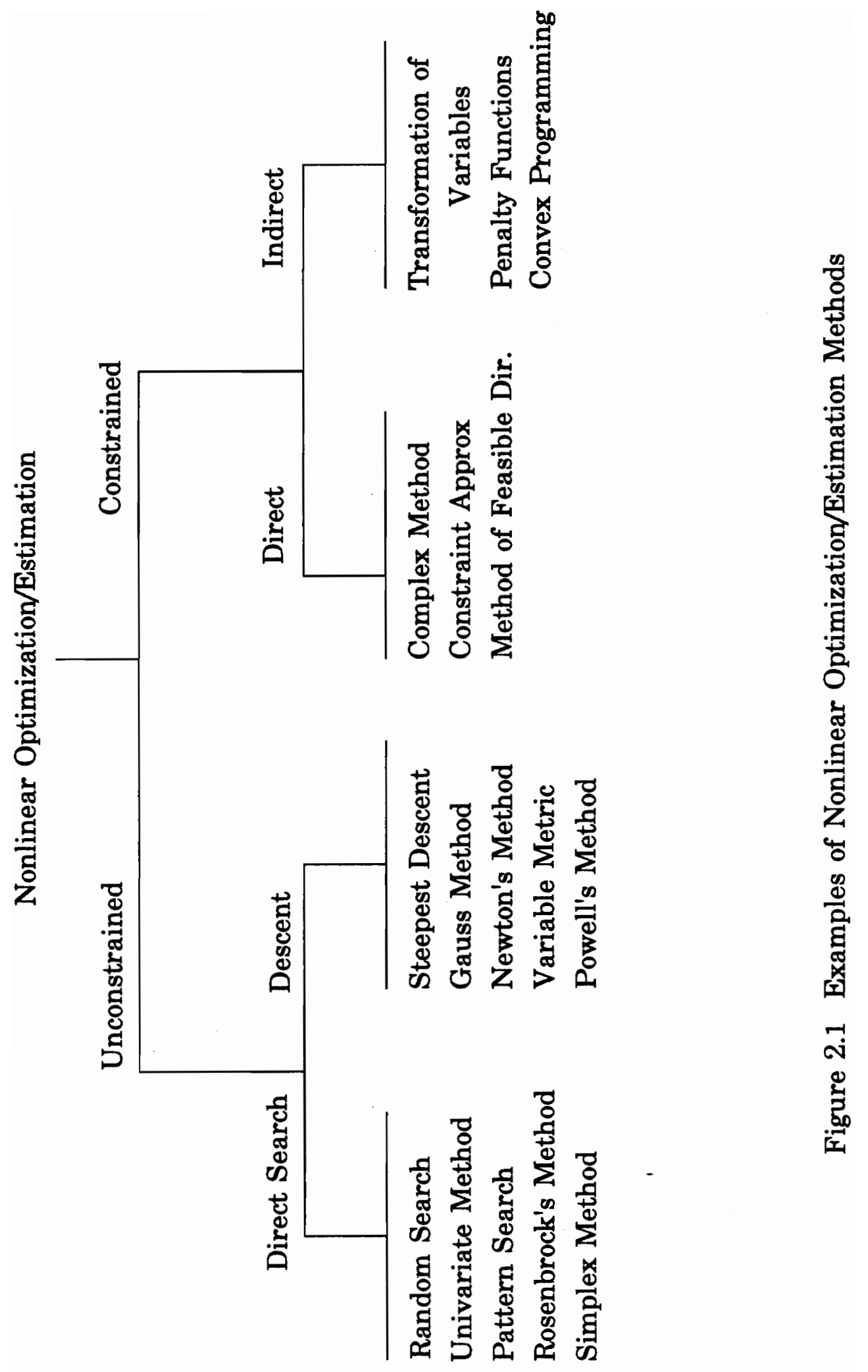


least squares is the easiest method to implement. It assumes that no prior information is know about the parameters being estimated and that the variances of measurement errors are generally unknown. Maximum likelihood estimation makes use of known information concerning the measurement errors. Maximum a posteriori estimation is Bayesian in nature and is used when the estimated parameters may be considered random variables or when prior information is known. Note that any parameter estimation routine could utilize any of the these estimators, it depends only on the form of the objective function. Due to a lack of prior information, ordinary least squares estimators were used in this study.

Two estimation procedures were used in this research. The first method which was used was the Gauss method. This is a first order unconstrained descent method which minimizes a least squares function containing differences between measured and calculated temperatures. It has the drawback of being inefficient for use in models which have correlated parameters or a nearly flat sum of squares function, as it may not converge. Box and Kanemasu (1972) proposed modifications to this method to change the step size of gradient information used in finding the estimates at each iteration in order to improve convergence. Bard (1974) later modified this method by making the requirement that the sum of squares function $S$, be strictly decreasing. The Gauss method and its derivatives are described in detail by Beck and Arnold (1977).

During the course of this study it was determined that constrained parameter estimation would be necessary. A penalty function method was used for this purpose. Penalty Function Methods provide constraints by assigning a numerical penalty to the objective function when a constraint is violated. The penalty is initially small and increases as the optimization progresses to prevent ill conditioning. More details on 
Penalty Function Methods and the Gauss method are given in Chapter 3.

The Gauss and Box-Kanemasu methods have been extensively used. Beck (1966) used the Gauss method to simultaneously estimate the thermal conductivity of nickel from transient temperature measurements. It was used successfully by Loh and Beck (1991) to estimate thermal conductivities in two directions, along with volumetric heat capacity. This study also included information from multiple sensors. Six thermal properties were estimated by Pfahl and Mitchell (1970) in a charring carbon-phenolic material. The Box-Kanemasu method was used by Moncman (1994), Hanak (1995), and by Moncman, Hanak, Copenhaver, and Scott (1995) for the estimation of thermal properties in carbon/epoxy composites. This technique has also been used in biomedical applications by Scott and Scott (1994), and in the freezing of basic food solutions by Saad and Scott (1994).

No cases could be found where penalty function methods or other constrained techniques were used to solve parameter estimation problems. However, these methods have been used to solve finite element models and other forward problems. Ezawa and Okomoto (1995) used penalty functions in conjunction with the finite element and boundary element methods to study elastic contact stresses. A similar approach was used by Fuehne and Engblom (1992) to predict stress fields in thick composite laminates. Zeng and Gullberg (1993) performed maximum a posteriori estimation to reconstruct transmission images from computed tomography. They used known attenuation coefficients of human tissue (skin, bones, etc.) as prior information. Their objective function was formed using a likelihood function of this attenuation coefficient data and a penalty function. The penalty function made sure that as the image was being constructed, the attenuation coefficients converged to their known values. Penalty 
functions were also used by Maubourguet-Pellerin and Pellerin (1987) to solve the Navier-Stokes equations for heat transfer and fluid flow through an interface.

\subsection{Property Estimation in Sandwich Structures}

References in the literature to thermal property estimation in honeycomb core sandwich structures are limited. Mechanical and thermal characteristics of these structures have been investigated on an analytical basis, with the majority of the analyses being mechanical. The incorporation of experimental data into estimation procedures has rarely been reported.

Farghaly and Shebl (1992) performed an analytical study on a sandwich beam with an elastically restrained end against both translation and rotation. They estimated effective end rigidity and end mass parameters by solving the sixth order DiTaranto/Mead-Marcus equation for transverse flexural displacement. A vibration analysis on a partially covered double sandwich cantilever beam was done by Chen and Levy (1994). They used Euler beam theory to derive the equations of motion for the system. They estimated the resonant frequency of the beam as well as system loss factors for different geometrical and physical properties.

One of the earliest works on the estimation of thermal properties in honeycomb core sandwich panels was done by Swann and Pittman (1961). They used a one dimensional finite difference heat transfer model which considered conductive and radiative effects. The model was steady-state in nature, and did not to treat the thermal capacitance of the structure. The core was analyzed on a unit cell basis, with each cell divided into ten elements. Constant temperature conditions were used at each boundary. 
The heat flux conducted through the structure was then calculated using known conductive and radiative properties of the structure material. An effective thermal conductivity was determined by Fourier's Law. A similar analysis was performed for corrugated core panels.

Other analyses of honeycomb-like structures exist. Radiation heat transfer in axisymmetric cylinders was modelled by Jamaluddin and Smith (1988). They examined radiation and the effects of scattering media in a coal-fired furnace. Asako (1990) has done extensive work on natural convection in air slots with a hexagonal honeycomb core. The honeycomb structure he analyzes has one face sheet fixed at a high temperature and the other at a low temperature. He solves for Nusselt numbers in the enclosure by using a power law control volume scheme developed by Patankar (1981).

Some references to honeycomb structures have been made in relation to home construction. A comparison of honeycomb core and foam core carbon-fibre/epoxy sandwich panels was made by Akay and Hanna (1990). A thermal analysis of a honeycomb roof cover system for energy conversion in an air-conditioned building was done by Kaushika et al. (1992). They analyzed radiation in the structure for the passive collection of solar energy and space heating. 


\section{CHAPTER 3}

\section{Theoretical Considerations}

The purpose of this chapter is to discuss the heat transfer model, estimation procedure, and experimental design used to determine the thermal properties of honeycomb-core sandwich structures. Recall that both measured and calculated data are needed for parameter estimation. The analytical aspects will be treated here, while experimental methods are discussed in Chapter 4.

The first section deals with the heat transfer model, which includes both radiative and conductive heat transfer. The model consists of a honeycomb structure with specified boundary conditions. A finite element code, Engineering Analysis Language (EAL version 330.07, by Whetstone, 1983), was used to solve this model due to the fact that nonlinear radiation terms make an exact solution unavailable. The next section describes the two methods of minimization that were used to estimate unknown properties. A modified Box-Kanemasu method is presented as well as an exterior penalty function method. The third section discusses confidence regions and intervals for the estimated parameters. The final section describes the procedure that was used to design the experiments. 


\subsection{Heat Transfer Model}

A mathematical model of the heat transfer through the honeycomb core structure was needed to determine temperature histories necessary for parameter estimation. In the estimation procedure, parameters in the model are changed until calculated temperatures most closely match experimental data in a least squares sense. The most simple model was chosen and then modified until it was sufficient to describe the heat transfer in the structure as explained in Chapter 4. It was necessary to provide a heat flux boundary condition in order to independently estimate all thermal properties. This has the effect of introducing Fourier's Law into the model:

$$
q^{\prime \prime}=-k \frac{\partial T}{\partial x}
$$

which provides information on $k$ independent of specific heat.

Figure 3.1 shows the heat transfer model and its boundary conditions. There are two distinct parts of the structure, the face sheet and core. The face sheet was modelled as a lumped capacitance only, with the volumetric specific heat to be determined. This was possible for two reasons. First, the face sheet thickness was negligible compared with the height of the core. Second, the aluminum/boron composite that composed part of the face sheet had a thermal conductivity which was an order of magnitude higher than the rest of the structure. This permitted quick diffusion of heat throughout the face sheet.

The core was modelled as a flat plate with resistance and capacitance and an area equal to that of one cell. Since the specific heat and thermal conductivity of the core 


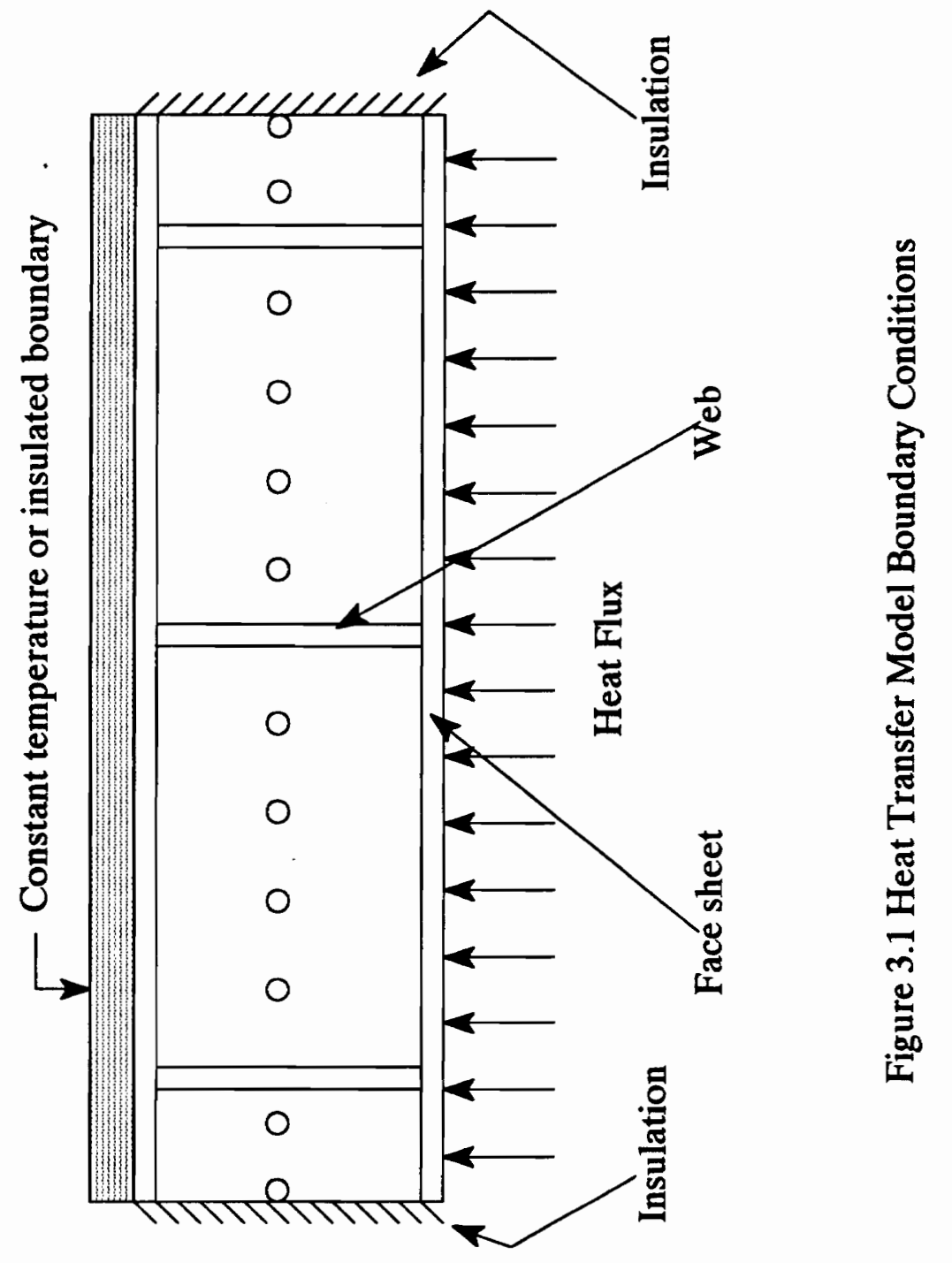


material, Ti-6Al-4V, is well known, attempting to estimate these properties would only serve to unnecessarily complicate the estimation procedure. The most important feature of the core is the wall thickness which varies due to the fabrication procedure, therefore, this was estimated instead of the specific heat and thermal conductivity of the core material. This variance includes a series of holes and welds. These features were not found to be a significant part of the heat transfer and were not modelled separately. Only an average wall thickness was estimated.

It was also desired to estimate the radiative characteristics of the structure. It was assumed that the interior of the core represented a diffuse, gray body. Therefore, only the total, hemispherical emissivity was estimated. A problem arose in how to model radiation heat transfer in a three dimensional enclosure while the conduction is modelled as one dimensional. The finite element code allowed for radiation view factors to be specified by the user. Radiation elements in the core were chosen to be equal area strips which ran horizontally from the bottom face sheet to the top, as shown in Figures 3.2a and 3.2b. Note that these elements include equal area portions of each side. They may be thought of as cylindrical bands which have been deformed into a square shape on the surface of the core. The height of each element corresponded to a conduction element. The part of the face sheets which enclosed the core were also treated as radiation elements.

In order to use radiation in the finite element program it was necessary to use the view factor method. The view factor $\mathrm{F}_{\mathrm{ij}}$ is defined as the fraction of radiant energy leaving surface $i$ which arrives at surface $j$. Several texts list formulas for simple three dimensional view factors. These, along with view factor algebra, could have been used to determine the view factors. However, this a time consuming process and also makes changing the 


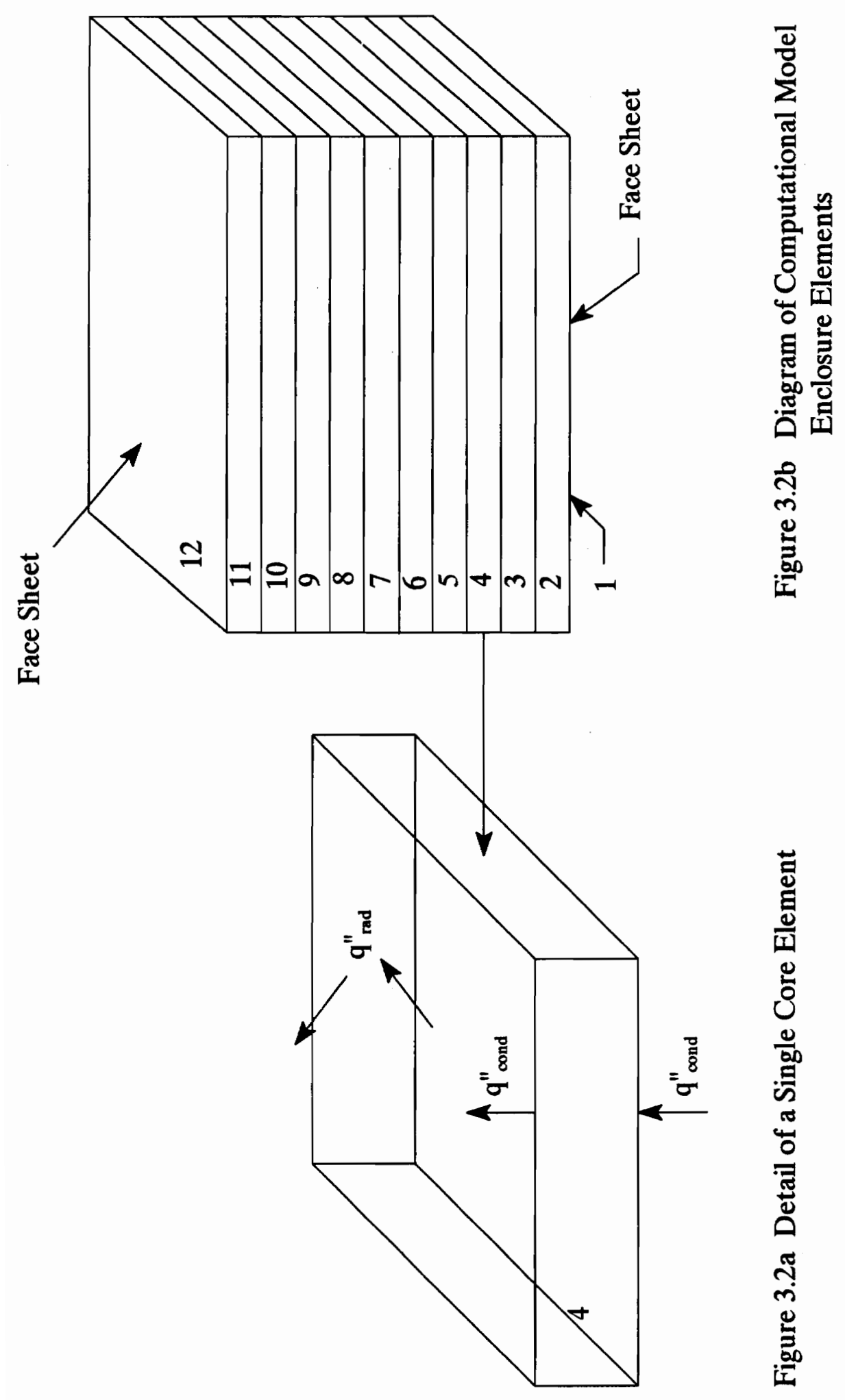


number of elements impractical.

A code was written which uses a Monte-Carlo method to calculate the view factors. The unit cell is divided into elements as shown in Figures 3.2a,b. Ten million rays are emitted from each of the core elements with a proportionally larger number emitted from the larger area of the face sheet. Coordinate axes were aligned in the plane of the bottom face sheet (Element 1) with one axis normal to the face sheet. The other two axes were placed co-planer with the face sheet and parallel to the sides of the enclosure. A random location of emission on the face sheet was chosen by generating three random numbers, one for each coordinate. For the vertical elements, two random numbers determined the location of emission. One number determined the height within the element, and the other determined the length from one corner. Two more random numbers were generated to determine a circumferential and azimuthal angle of emission.

Because of the geometry of the core, it was necessary to determine the intersection of each emitted ray with each plane of the core. The actual point of intersection was the one which was located within the dimensions of the enclosure. The specific element could then be determined by its distance from the bottom face sheet. A counter was incremented each time an element was hit by an emitted ray. The fraction of rays intercepted by surface $j$ and emitted by surface $i$ is the view factor $F_{i j}$ as previously defined.

View factors were calculated by emitting rays from the bottom face sheet element and half of the core elements. It was only necessary to emit rays from half of the elements because the enclosure is symmetric from top to bottom. The remaining view factors were assigned the same values as their symmetric counterpoints. A listing of the view factors is shown in Table 3.1. In this table the element from which energy is 


\begin{tabular}{|c|c|c|c|c|c|c|c|c|c|c|c|c|c|}
\hline & $\approx$ & 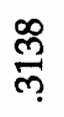 & 芯 & $\vec{F}$ & $\underline{\overrightarrow{0}}$ & $\mid$\begin{tabular}{l}
$\infty$ \\
\hdashline \\
\hdashline \\
\hdashline
\end{tabular} & 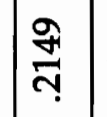 & 勇 & 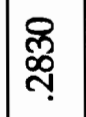 & 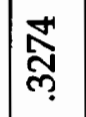 & 怘 & 容 & 8 \\
\hline & $\Rightarrow$ & 离 & 气̊ & 宛 & శ్శ & 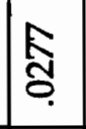 & $\mid \frac{\infty}{\bar{\rho}}$ & 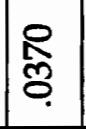 & 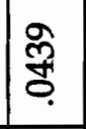 & 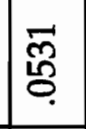 & 尺ั. & ळ & ఫ̊ \\
\hline & $\because$ & 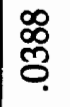 & ర్t & శ్శ్ర & 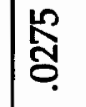 & 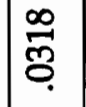 & 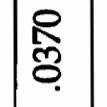 & 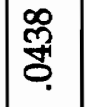 & 䓀 & 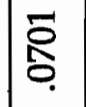 & 吾 & \&్ & \% \\
\hline & $a$ & ț. & 岗 & 㝕 & 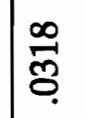 & 倁 & 产 & 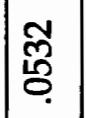 & \begin{tabular}{|l|}
$\infty$ \\
0 \\
8 \\
0
\end{tabular} & 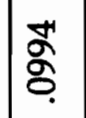 & $\begin{array}{l}\text { ôे } \\
\text { o. }\end{array}$ & 宽 & F. \\
\hline & $\infty$ & $\begin{array}{l}\text { 总 } \\
\text { o. }\end{array}$ & 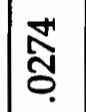 & 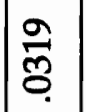 & 魚 & 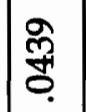 & 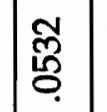 & 鸽 & \begin{tabular}{|l|} 
\\
$\sigma$ \\
$\sigma$
\end{tabular} & 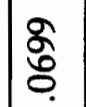 & $\overrightarrow{0}$ & 㞼 & 管 \\
\hline 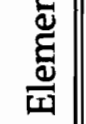 & $\wedge$ & $\begin{array}{l}\text { 莣 } \\
\text { o. }\end{array}$ & $\frac{\partial}{\bar{\partial}}$ & 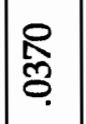 & 文 & 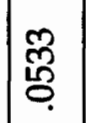 & $\begin{array}{l}8 \\
8 \\
5\end{array}$ & \begin{tabular}{|l} 
20 \\
o
\end{tabular} & $\begin{array}{l}\overline{8} \\
\text { o. }\end{array}$ & 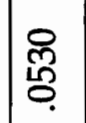 & 粍 & 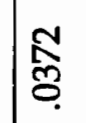 & R \\
\hline 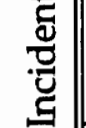 & 0 & 尽 & $\mid \begin{array}{c}\mathbb{N} \\
\text { Oo } \\
\end{array}$ & 窎 & 居 & 容 & 吕 & 竞 & 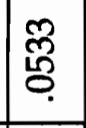 & 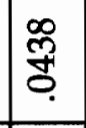 & $\begin{array}{l}0 \\
\text { Oִ } \\
\text { o }\end{array}$ & है. & $\begin{array}{l}\text { 贺 } \\
\text { 号. }\end{array}$ \\
\hline & in & E् & 孪 & \begin{tabular}{|l|}
$\vec{p}$ \\
岁.
\end{tabular} & 8. & $\mid$ & $\mid \begin{array}{l}\hat{2} \\
\overline{0}\end{array}$ & 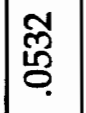 & 害 & 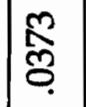 & $\frac{\partial}{\bar{\varnothing}}$ & 㚃 & $\begin{array}{l}0 \\
0 \% \\
0 \\
0 .\end{array}$ \\
\hline & + & 庹 & \begin{tabular}{|l|}
$\bar{m}$ \\
苂.
\end{tabular} & 鸽 & 荾 & $\begin{array}{l}\infty \\
0 \\
0 \\
0\end{array}$ & 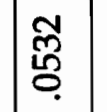 & 商 & 倁 & $\begin{array}{l}\infty \\
\bar{\varnothing} \\
.\end{array}$ & 太્ત & 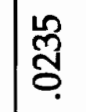 & 亲 \\
\hline & $m$ & 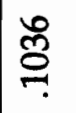 & $\begin{array}{l}8 \\
8 \\
0\end{array}$ & 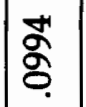 & 8 & 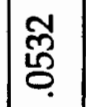 & 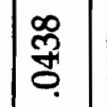 & 尺. & $\frac{\infty}{\bar{o}}$ & 蛋 & ڤે & 苛 & 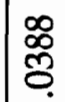 \\
\hline & $\mathbf{N}$ & శ్ & \% & 尺̊: & 苟 & 嵩 & 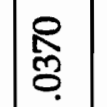 & 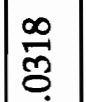 & E్త్ర & 苞 & ఫ્ठ & 总 & F \\
\hline & -7 & 8 & $\mid \overrightarrow{5}$ & 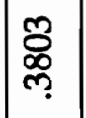 & 촗 & 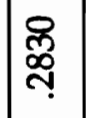 & $\mid \begin{array}{l}\overrightarrow{0} \\
\text { di }\end{array}$ & $\underset{\mathrm{T}}{\stackrel{g}{\mathrm{~T}}}$ & \begin{tabular}{l}
$\infty$ \\
$\infty$ \\
$\infty$ \\
\hdashline \\
\hdashline
\end{tabular} & $\overrightarrow{\widetilde{W}}$ & $\vec{F}$ & 晜 & 免 \\
\hline 㽦 & & - & N & $m$ & $\pi$ & 10 & 0 & In & $\infty$ & 10 & $\stackrel{9}{9}$ & $=$ & $\approx$ \\
\hline
\end{tabular}


emitted is listed in the vertical column on the right, while the incident elements are listed horizontally.

The view factor from one face sheet to another was most easily calculated. It was checked against the exact solution (Incropera, 1985):

$$
\begin{aligned}
& F_{i j}=\frac{2}{\pi \bar{X} \bar{Y}}\left\{\ln \left[\frac{\left(1+\bar{X}^{2}\right)\left(1+\bar{Y}^{2}\right)}{1+\bar{X}^{2}+\bar{Y}^{2}}\right]^{1 / 2}+\bar{X}\left(1+\bar{Y}^{2}\right)^{1 / 2} \tan ^{-1} \frac{\bar{X}}{\left(1+\bar{Y}^{2}\right)^{1 / 2}}\right. \\
& \left.+\bar{Y}\left(1+\bar{X}^{2}\right)^{1 / 2} \tan ^{-1} \frac{\bar{Y}}{\left(1+\bar{X}^{2}\right)^{1 / 2}}-\bar{X} \tan ^{-1} \bar{X}-\bar{Y} \tan ^{-1} \bar{Y}\right\}
\end{aligned}
$$

Here $\bar{X}$ and $\bar{Y}$ are the ratios of the transverse dimensions to the vertical dimension. The exact solution gave a value of 0.3134 . The Monte Carlo solution yields 0.3138 , a difference of only $0.13 \%$ from the exact. Also note that each row in Table 3.1 sums to 1.000 at three or four significant digits. This shows that energy is conserved in the enclosure and that all rays are accounted for. These facts lend credibility to the calculated view factors.

Although air is present in the core, it does not significantly contribute to the heat transfer in the structure. Scott (1994) performed an analytical study on the structure and found that the thermal resistance of heat transfer modes involving air was on the order of ten times that of the core resistance. Therefore, both heat conduction through the air and free convection in the cell enclosures were neglected.

As previously stated, EAL was used to numerically solve for the temperature 
distribution in the structure. EAL solves the following matrix equation

$$
[C+\beta \Delta t K] T_{i+1}=[C-(1-\beta) \Delta t K] T_{i}+F \Delta t+F \beta \Delta t^{2}
$$

In the above equation $C$ is the capacitance matrix, $K$ is the resistance load vector, $T_{i}$ is the temperature vector at time $t_{i}$, and $T_{i+1}$ is the temperature vector at time $t_{i+1}$. The weighting function, $\beta$, was set to 0.5 in order to implement the Crank-Nicolson algorithm. The load vector $K$, contains both conductive and radiative terms:

$$
K=K_{k}+4 \tilde{K} T_{o}^{3}
$$

The vector $F$ and its time derivative both contain information specifying the boundary conditions. All load vectors and temperatures were computed at time steps corresponding to times at which experimental data were taken.

Only ten elements through the thickness were used. Adding additional elements did not significantly change the resulting temperature histories. There were two additional elements that were used to model the face sheets. All twelve elements had radiation exchange.

\subsection{Minimization Procedure}

The parameter estimation problem was solved by minimizing an objective function. This function is a sum of squares function described by 


$$
S=[\boldsymbol{Y}-\eta(\boldsymbol{\beta})]^{T}[\boldsymbol{Y}-\eta(\boldsymbol{\beta})]
$$

where $\boldsymbol{Y}$ is a vector of measured temperatures, $\boldsymbol{\eta}$ is the vector of calculated temperatures (from EAL). It is a function of $\boldsymbol{\beta}$, the parameters to be estimated.

Another method that could have been used involves constraining $\boldsymbol{\eta}$ to be within a certain $\delta$ of $\mathbf{Y}$ for all $t$. This would eliminate some problems which could be associated with a very non-linear $S$, however it would be much more difficult to implement.

Two methods of minimizing $S$ were used in this study. This first was a modified Box-Kanemasu method. It has been a common method of parameter estimation in heat transfer. This method however, did not converge and was abandoned. A more general optimization method was used which allowed the parameters to be estimated while being constrained.

\subsubsection{Modified Box-Kanemasu Method}

The Box-Kanemasu method is a modification of the Gauss linearization method. The Gauss method specifies a direction and step size that the parameter vector should be changed in order for $\mathrm{S}$ to be a minimum. One condition necessary at the minimum of $S$ is that the derivative of $S$ with respect to the parameter vector is zero:

$$
\nabla_{\beta} S=2\left[-\nabla_{\beta} \eta^{T}(\beta)\right][Y-\eta(\beta)]=0
$$

The derivative of $\boldsymbol{\eta}$ with respect to $\boldsymbol{\beta}$ is called the sensitivity matrix and is donated by 
$\boldsymbol{X}(\boldsymbol{\beta})$. Each component of this matrix is a sensitivity coefficient. The sensitivity coefficients relate how the dependent variable (temperature) changes with respect to the parameters being estimated. Sensitivity coefficients will later be used to give a graphical description of the effect of each parameter on the model.

The solution to the heat transfer problem studied here is inherently nonlinear in terms of temperature. This leads to difficulty when solving for $\boldsymbol{\beta}$. Therefore, two approximations are used which permit an easier solution. The first involves replacing $\boldsymbol{X}(\boldsymbol{\beta})$ by $\boldsymbol{X}(\boldsymbol{b})$, where $\boldsymbol{b}$ is an estimate of $\boldsymbol{\beta}$. The second uses the first two terms of the Taylor series of $T(\boldsymbol{\beta})$ about $\mathbf{b}$ to approximate $T(\boldsymbol{\beta})$. The simplifications lead to the following solution for $\mathbf{b}$ :

$$
b^{(k+1)}=b^{(k)}+P^{(k)}\left[X^{T(k)}\left(Y-T^{(k)}\right)\right]
$$

where

$$
P^{-1(k)} \equiv X^{T(k)} X^{(k)}
$$

and $k$ is the iteration index. This is known as the Gauss method. Note that iteration on $k$ is required for nonlinear-in-parameter models. This method may not converge for some nonlinear models. A modification of this method developed by Box and Kanemasu modifies the step size at each iteration in order to improve convergence. A further modification requires that the sum of squares function, $S$, continuously decreases. The following equations are the result of these modifications (Beck and Arnold, 1977):

$$
b^{(k+1)}=b^{(k)}+h^{(k+1)} \Delta_{g} b^{(k)}
$$

where 


$$
\Delta_{g} b^{(k)}=P^{(k)}\left[X^{T(k)}\left(Y-\eta^{(k)}\right)\right]
$$

and

$$
h^{(k+1)}=G^{(k)} \alpha^{2}\left[S_{\alpha}^{(k)}-S_{o}^{(k)}+2 G^{(k)} \alpha\right]^{-1}
$$

where

$$
G^{(k)}=\left[{ }_{g} b^{(k)}\right]^{T}\left(X^{T(k)} X^{(k)}\right)\left[{ }_{g} b^{(k)}\right]
$$

and $h$ is a quadratic approximation of $S$. The parameter $\alpha$ is used to ensure that $S$ continually decreases.

The Box-Kanemasu method does not work well when the estimated parameters are correlated. This means that the sensitivity coefficients are almost linearly dependent and the sum of squares function has no unique or well defined minimum. The approximate correlation matrix, $r$, is defined by Beck and Arnold (1977) to be

$$
r_{i j}=P_{i j}\left(P_{i i} P_{i j}\right)^{-1 / 2}
$$

This matrix is symmetric with the diagonal terms being unity and the off-diagonal terms being between -1 and 1 . Values of these terms near 1.0 or -1.0 indicate linear dependence. Beck states that difficulty in estimating properties may occur when the terms exceed $0.9(-0.9)$.

In this study it was found that correlation between the wall thickness and 
emissivity were on the order of 0.999 . The Box-Kanemasu method, or any method from which it was derived, failed to converge for all test cases. Usually one of the estimated properties would become negative, causing the finite element code to terminate execution.

The results of using the Box-Kanemasu algorithm indicated that a method is needed which allows the parameters to be constrained in some sense. While using a different model for $\eta$ might alleviate part of the problem, the parameters estimated in this study represent modes of heat transfer which are known to occur. The need to constrain parameters requires the use of more general optimization techniques which will be discussed in the next section.

\subsubsection{Constrained Parameter Estimation}

The purpose of the Box-Kanemasu algorithm was to reduce a sum of squares function to a minimum. The sum of squares function, $S$, could be treated as an objective function. This function could be minimized by a general optimization routine. Most optimization techniques allow for constraints and nonlinear parameters. Examples of such methods are sequential linear programming, penalty function methods, and Lagrange multiplier methods.

The Exterior Penalty Function strategy was used for this study because it is relatively simple to incorporate into the optimization process. It treats the objective function as an unconstrained function but provides a penalty to limit constraint violations. The imposed penalty is initially small but increases with each iteration in order to prevent ill-conditioning. This process requires the solution of several 
unconstrained problems in order to obtain the optimum constrained solution.

In order to include the penalty in the optimization procedure a pseudo-objective function is created. It is of the form

$$
\Phi\left(\chi, r_{p}\right)=F(\chi)+r_{p} P(\chi)
$$

where $\chi$ represents the vector of independent variables, $F(\chi)$ is the original objective function, $P(\chi)$ is the imposed penalty, and $r_{p}$ is a variable which scales the magnitude of the penalty. The penalty function is of the form

$$
P(\chi)=\sum_{j=1}^{m}\left[\max \left[0, g_{j}(\chi)\right]\right]^{2}
$$

where $g_{j}(\chi)$ represents inequality constraints. Equality constraints could also be included but none were needed. Note that if no constraints are violated there is no penalty. When a penalty does occur, the violation is squared to provide a zero slope for the penalty at the constraint boundary. This gives the pseudo-objective function a continuous slope. The algorithm is shown in Figure 3.3. The constant $\gamma$ is used to vary $r_{p}$ after each iteration. Convergence is achieved when $F(\chi)$ does not change significantly from one iteration to the next and the constraints are also satisfied within a given tolerance.

A strategy has been outlined which allows the incorporation of constraints into the objective function. The next step is to find the minimum of the new objective function $\Phi\left(\chi, r_{p}\right)$. This means that

$$
\nabla \Phi\left(\chi, r_{p}\right)=0
$$




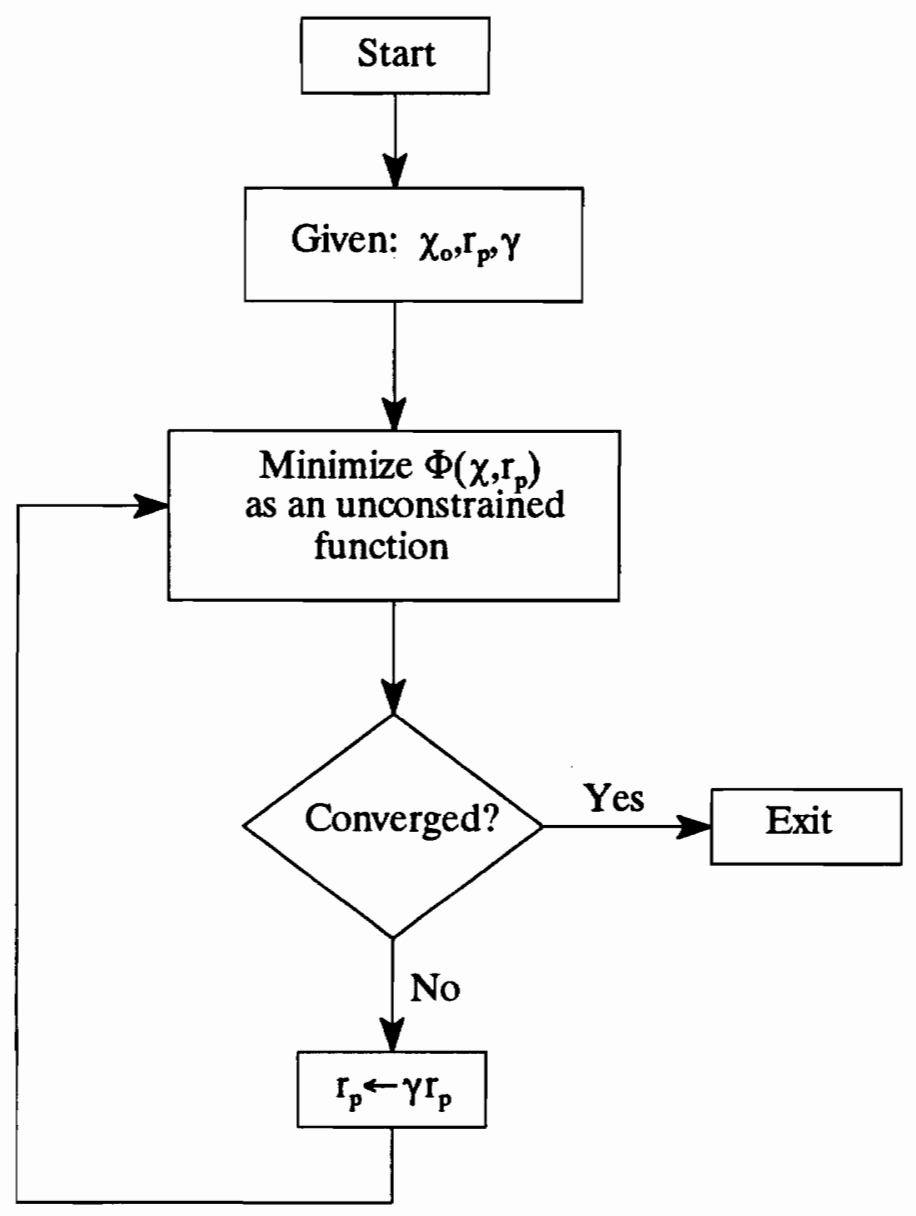

Figure 3.3 Exterior Penalty Function Algorithm 
or the gradient of $\Phi$ is zero. The are several ways of doing this, but first order methods are probably the most efficient. Figure 3.4 shows the algorithm for unconstrained minimization.

One class of first order methods are called variable metric methods. These methods carry prior information in an n-dimensional ( $\mathrm{n}$ parameters) array to update the search direction at each iteration. A one-dimensional search is then performed in each direction to find the minimum of the objective function. The search direction, $\mathbf{S}$, at each iteration $k$ is

$$
S^{(k)}=-H \nabla \Phi\left(\chi^{(k)}, r_{p}\right)
$$

This equation results from taking the second-order Taylor series expansion of the objective function about $\chi^{\circ}$, differentiating with respect to $\chi$, and then setting the result equal to zero. The matrix, $H$, approximates the inverse of the Hessian matrix. The Hessian matrix contains the second partial derivatives of the objective function with respect to the design variables. This gives these methods some second order or quasiNewton characteristics. Initially $H$ is chosen to be the identity matrix. Therefore the initial search direction is that of steepest descent. Note that $\boldsymbol{H}$ is derived from first-order information and that is why this isn't a true second-order method. Direct calculation and inversion of the actual Hessian matrix would be computationally expensive. At each iteration $\boldsymbol{H}$ is updated as follows:

$$
H^{(k+1)}=H^{(k)}+D^{(k)}
$$




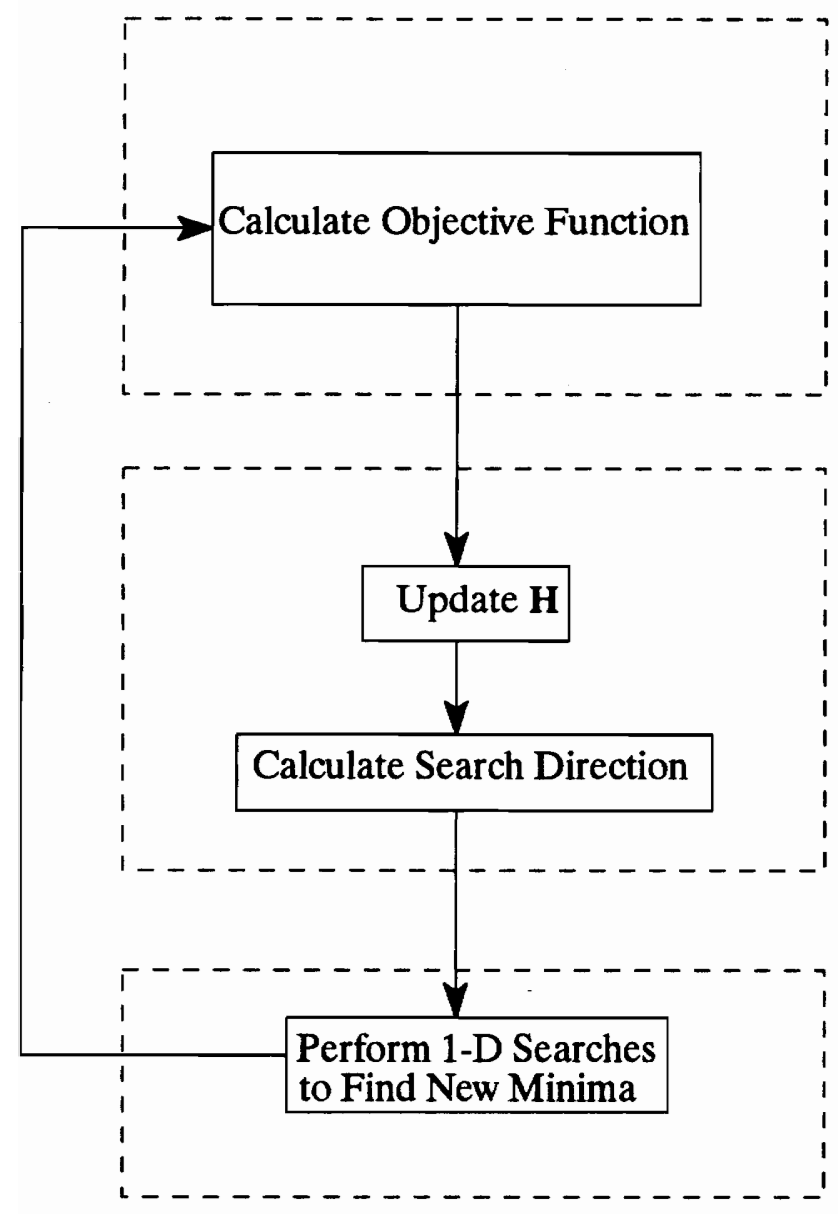

Exterior Penalty Function Strategy

Determination of Search Direction BFGS Method

\section{Polynomial} Interpolation

Figure 3.4 Unconstrained Minimization Algorithm 
and $D$ is an update matrix of the form (Vanderplaats, 1984):

$$
D^{(k)}=\frac{\sigma+\theta \tau}{\sigma^{2}} p p^{T}+\frac{\theta-1}{\tau} H^{(k)} y\left(H^{(k)} y\right)^{T}-\frac{\theta}{\sigma}\left[H^{(k)} y P^{T}+p\left(H^{(k)} y\right)^{T}\right]
$$

The vectors $\mathbf{p}$ and $\mathbf{y}$ are defined as

$$
\begin{gathered}
p=\chi^{(k)}-\chi^{(\mathbf{R}-1)} \\
y=\nabla \Phi\left(\chi^{(k)}, r_{p}\right)-\nabla \Phi\left(\chi^{(k-1)}, r_{p}\right)
\end{gathered}
$$

and $\sigma$ and $\tau$ are defined as

$$
\begin{gathered}
\sigma=p \cdot y \\
\tau=y^{T} H^{(k)} y
\end{gathered}
$$

The variable, $\theta$, can be any number between 0 and 1 . For this study it was set equal to 1. This is commonly referred to as the Broyden-Fletcher-Goldfarb-Shanno (BFGS) method. The vectors $p$ and $y$ are used to build up second order derivative information which changes from iteration to iteration as $X$ moves in the design space. The variable, $\theta$, has the effect of describing how the information is updated. More information is given in Vanderplaats, 1984.

The final step in this procedure is to perform a one-dimensional search to determine the minimum of the objective function. This has the effect of cutting a plane through the design space along the search direction. The objective function is now only 
a function of one variable which relates to the position in design space along the search direction. The one-dimensional search is used here to find the minimum of the objective function in this plane. Polynomial interpolation was used as the means of minimization. The above optimization equations were part of the Engineering Analysis Language finite element code. The use of EAL will be described in more detail in Chapter 5.

\subsection{Confidence Regions and Intervals}

Confidence regions and intervals are useful in describing how accurately the estimated parameters are known. Confidence intervals refer to how well a specific parameter may be known without regard to the other parameters. Confidence regions show how the uncertainties in the parameters interact. The result is graphically, a hyperellipsoid in $n$-space.

Confidence intervals for each parameter can be calculated by the following

$$
\beta_{k}=b_{k} \pm\left[P_{k k} \frac{S}{N-p}\right]^{1 / 2} t_{1-\alpha / 2}(N-p)
$$

where $S$ is the sum of squares, $N$ is the number of measurements, and $p$ is the number of parameters. This confidence interval uses the Student's - $t$ distribution, although the size of $N$ for these experiments would allow for the use of the normal distribution. There several assumptions which pertain to using this confidence interval: additive errors with zero mean, normally distributed errors, nonstochastic independent variables, and no prior 
information regarding the parameters.

A formulation for the confidence region is as follows (Beck and Arnold, 1977)

$$
(b-\beta)^{T}(P)^{-1}(b-\beta)=p s^{2} F_{1-\alpha}(p, n-p)
$$

This is the equation of a hyperellipsoid centered at the origin with coordinate axes $\boldsymbol{b}_{\boldsymbol{i}}$ $\boldsymbol{\beta}_{i}$ representing uncertainty in each parameter, $i$. The statistic $F_{1-\alpha}$ represents the $\mathrm{F}$ distribution.

\subsection{Design of Experiments}

Many criteria have been proposed for the design of experiments. They generally include some form of the $\boldsymbol{X}^{T} \boldsymbol{X}$ matrix, where $\boldsymbol{X}$ is the sensitivity matrix. These include maximization of the determinant of $\boldsymbol{X}^{T} \boldsymbol{X}$ (D-optimal), maximization of the minimum eigenvalue of $\boldsymbol{X}^{T} \boldsymbol{X}$ (C-optimal), and maximization of the trace of $\boldsymbol{X}^{T} \boldsymbol{X}$ (A-optimal). The first criteria is the most common one. It will minimize the hypervolume of the confidence region, but may do so at the expense of creating a large error in one of the parameters. Maximizing the minimum eigenvalue appears to be a better idea but does still not address the inherent relationship that exists between all parameters.

In the end, it is desirable to make all the confidence intervals and regions as small as possible. A criteria is defined which seeks to minimize the largest confidence interval. The confidence interval as previously defined represents the maximum length of the coordinate axes of the confidence region hyperellipsoid. This can be shown by using Lagrange Multipliers.

The confidence region was defined by Eq. 3.25 . First replace $(\mathbf{b}-\boldsymbol{\beta})$ in Eq. 3.25 by 
$\mathbf{x}$ for simplicity. Also replace the right-hand side by $r^{2}$. The equation can be rewritten as

$$
x^{T} P^{-1} x=r^{2}
$$

It is desired to find the maximum of some component, $x_{1}$, on Eq. 3.26. The Lagrangian is

$$
L=x_{1}+\lambda\left(x^{T} P^{-1} x-r^{2}\right)
$$

To maximize, set the first derivative with respect to $x$ equal to zero

$$
\left(\frac{\partial L}{\partial x}\right)^{T}=0
$$

This expression becomes

$$
0=\left[\begin{array}{c}
1 \\
0 \\
0 \\
\vdots \\
0
\end{array}\right]+2 \lambda P^{-1} x
$$

Multiplying by $\boldsymbol{x}^{T}$,

$$
0=x_{1}+2 \lambda x^{T} P^{-1} x
$$

Solving this equation for $\lambda$ gives 


$$
\lambda=\frac{-x_{1}}{2 r^{2}}
$$

Substituting back into Eq. 3.28

$$
0=\left[\begin{array}{c}
1 \\
0 \\
0 \\
\vdots \\
0
\end{array}\right]+\frac{-x_{1}}{r^{2}} P^{-1} x
$$

Solving for $x_{1} x$

$$
x_{1} \boldsymbol{x}=r^{2} \boldsymbol{P}\left[\begin{array}{c}
1 \\
0 \\
0 \\
\vdots \\
0
\end{array}\right]
$$

In matrix form this is equivalent to

$$
\left[\begin{array}{c}
x_{1}^{2} \\
x_{1} x_{2} \\
\vdots \\
x_{1} x_{n}
\end{array}\right]=r^{2}\left[\begin{array}{c}
P_{11} \\
P_{22} \\
\vdots \\
P_{n n}
\end{array}\right]
$$

Equating the terms in the first row

$$
x_{1}=\sqrt{r^{2} P_{11}}
$$

$\mathbf{r}^{2}$ contains the sum of squares, $t$ statistic, and number of degrees of freedom as shown in (3.26). 
Recall that the confidence interval contains the diagonal terms of the $\boldsymbol{X}^{T} \boldsymbol{X}$ matrix. The diagonal terms are of the following form

$$
\boldsymbol{P}_{k k}=\sum_{j=1}^{p} \frac{e_{j k}^{2}}{\lambda_{j}}
$$

where $e_{j k}$ and $\lambda_{j}$ are the eigenvectors and eigenvalues of $\boldsymbol{X}^{T} \boldsymbol{X}$, respectively. The eigenvectors and eigenvalues geometrically represent the principle lengths and directions of the confidence region. If $S$ in Eq. (3.24) is replaced by a constant (possibly measurement error) then the confidence interval could be used as a design criteria. This criteria will be called the scaled confidence interval approach. 


\section{CHAPTER 4}

\section{Experimental Procedures}

Parameter estimation closely resembles inverse problems in the sense that both measured and calculated data are needed. Measured temperatures are compared to numerically simulated data in a least squares sense. There are two requirements for the simultaneous estimation of both thermal conductivity and specific heat. The temperature response of the sample must be transient, and the heat flux at one or more boundaries must be specified. These conditions, along with optimal experimental design, were incorporated into the experiments described in this chapter. All experiments were performed at the NASA-Langley Research Center.

The first section describes the physical aspects of the experiment. This includes the actual samples that were tested, as well as the data acquisition equipment and instrumentation needed in the experiment. The second section deals with thermal visualization of the sample. This section provides a verification of the analytical model that was chosen. The next section presents a list of experiments. The last section focuses on the experimental data and how it was incorporated into the estimation program. 


\subsection{Experimental Apparatus}

Experiments were required which provided a constant heat flux at one boundary while maintaining known conditions at all others. Temperature histories were recorded at both surfaces. The sensors and equipment necessary to perform these operations are detailed in the following sub-sections. A description of the test samples, their dimensions and construction, are also given. The second sub-section describes the test apparatus. This includes the test fixture, generation of heat flux, and the other boundary conditions. Information is given on the temperature sensors that were used and the instrumentation needed to run them. The final sub-section gives an overview of the remaining parts of the experiment, such as the oven used to provide the initial temperature and the data acquisition system.

\subsubsection{Test Samples}

The samples being analyzed are SPF/DB (Super Plastic Formed, Double Bonded) 4 sheet honeycomb core sandwich made by McDonnell Douglas. The core material is Ti$6 \mathrm{AL}-4 \mathrm{~V}$. The face sheets are made of an aluminum - boron fiber composite. Figure 4.1 shows the manufacturing process.

The process begins by spot-welding a grid pattern on two sheets which will comprise the core. A gas pressure tube is attached to one corner. The core undergoes plastic expansion under high temperature until it reaches the face sheet and limiting fixture. One result of this process is that the core is left with a non-uniform wall thickness, as well as a series of holes and spot-welds at the centerline. 


\section{Honeycomb Core Phases of Manufacturing}

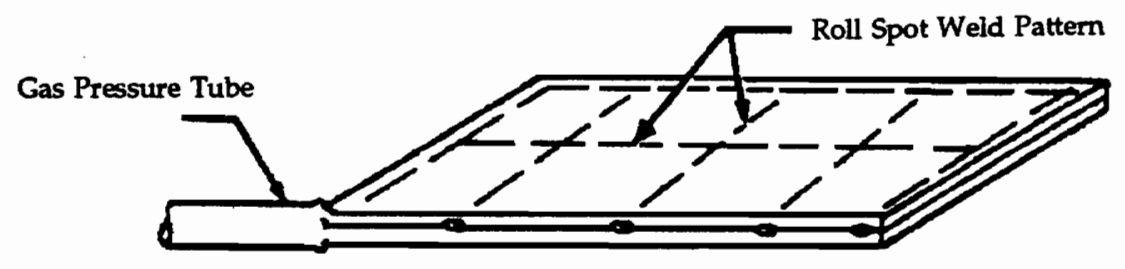

Envelope (Sectioned) Before Superplastic Forming

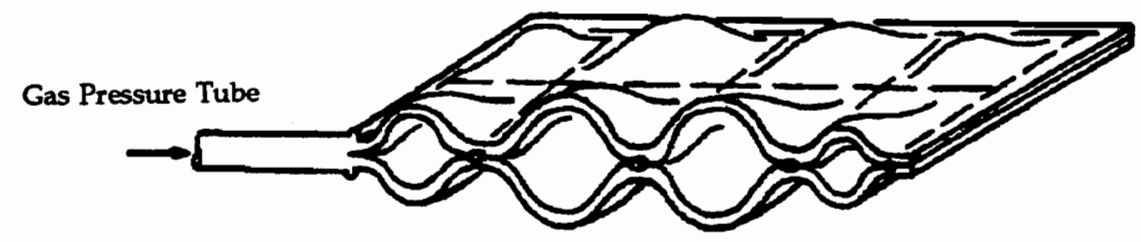

Envelope (Sectioned) Partially Formed

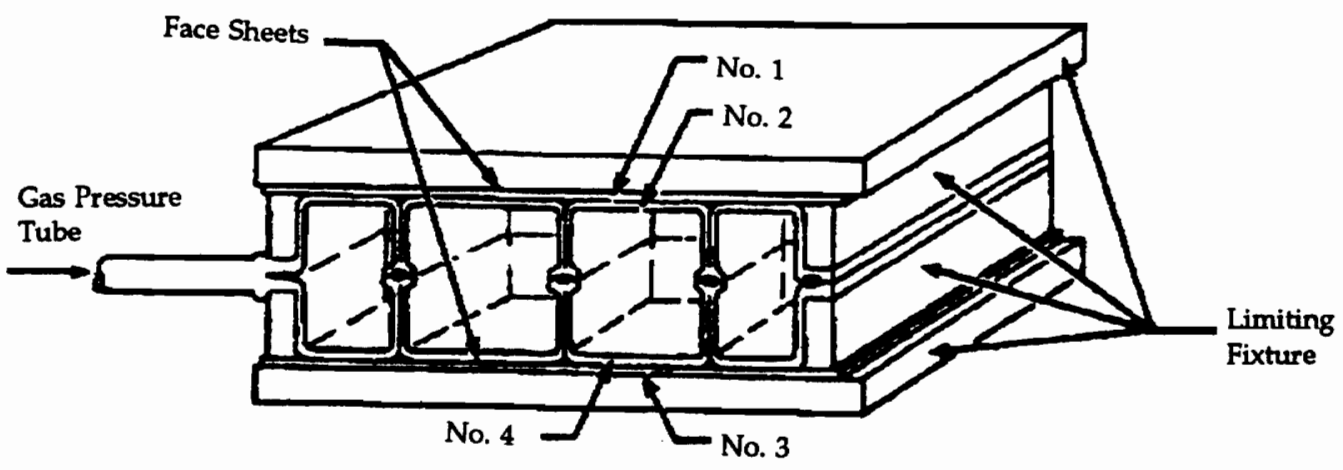

Envelope (Sectioned) With Face Sheets Forming Complete Sandwich

Figure 4.1 Honeycomb Core SPF/DB Phases of Development 
Nominal values for the core web thickness, face sheet thickness, and unit cell dimensions were measured. A diagram of the core, face sheets, and their measurements is shown in Figure 4.2.

\subsubsection{Experiment Configurations}

This section describes the experimental apparatus and how it was built. The experimental apparatus was symmetric with respect to the heater. A sample was placed on either side of the heater. An RTD was placed between the two to measure the temperature at the heated surface. Either insulation or a large aluminum block was placed on the other side of the sample. An RTD was inserted here as well, to measure the boundary temperature.

One sensor was required at each surface. The heated surface temperature history was to be the main source of data in the estimation procedure. It was also necessary to record the temperature at the other surface. For the constant temperature configuration, the aluminum blocks did not act as perfect heat sinks. There was a small temperature rise which was recorded with an RTD and incorporated into the estimation program as a variable temperature boundary. The boundary temperature was recorded for the insulated case because this information could be directly used in the estimation program.

The apparatus was built starting with the heater which had the same length and width as the samples. A thin layer of Thermal Joint Compound (EG\&G Wakefield Engineering Co.) was placed between the heater and RTD, as well as between the RTD

and sample. The purpose of this was to reduce contact resistance. Another layer of grease was placed between the sample and the next RTD. For the constant temperature 

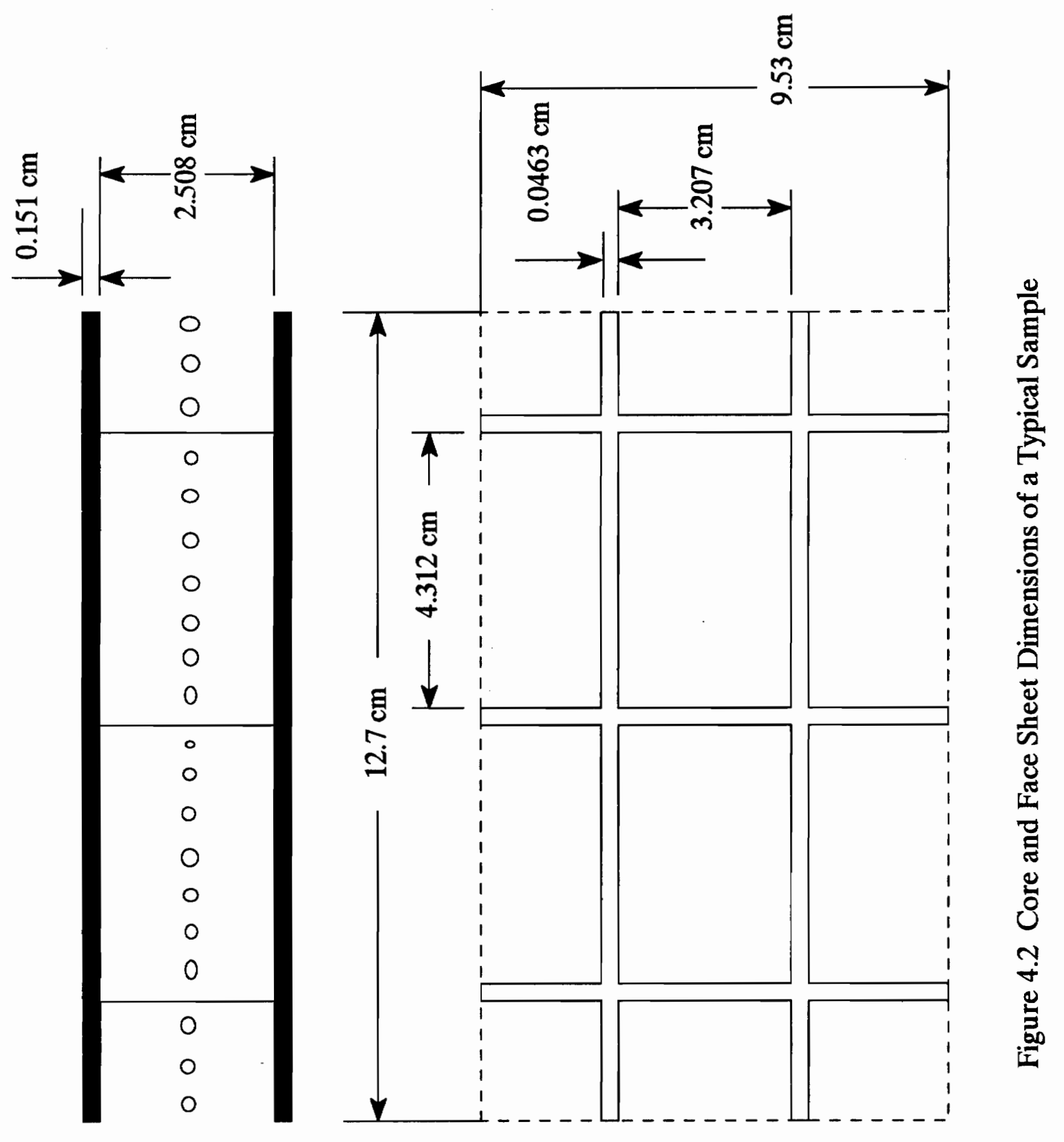
boundary another layer of thermal grease was placed between the second RTD and the aluminum block. No thermal grease was used for the insulated boundary since contact resistance would be desirable here. Schematics of the two configurations are shown in Figures $4.3 \mathrm{a}$ and $4.3 \mathrm{~b}$.

Aluminum blocks for the constant temperature boundary measured $9.53 \mathrm{~cm} . \mathrm{x}$ $12.7 \mathrm{~cm} . \times 7.62 \mathrm{~cm}$. Aluminum was chosen because of its high thermal conductivity. Copper would have been a better choice, but it was not possible to find two pieces large enough. The insulation for the insulated boundary was $9.53 \mathrm{~cm} . \times 12.7 \mathrm{~cm} \times 5.08 \mathrm{~cm}$. thick ( 360 Standard Ceramic Board by Cotronics Corporation) with thermal conductivity of $0.45 \mathrm{BTU}$ in $/ \mathrm{hr}^{\circ} \mathrm{F} \mathrm{ft}$ and specific heat of $0.25 \mathrm{BTU} / \mathrm{lb} \mathrm{F}$. The edges of the insulation were wrapped with aluminum tape to keep the board from coming apart. Care was taken to assure that no tape touched the samples. This same insulation was placed around the edges of the sample to prevent heat loss at the edges which would have caused in-plane thermal gradients. The insulation was held against the apparatus with wire to insure good contact.

\subsubsection{Experimental Setup}

The electrical resistance heater used in this experiment was made by Minco Products, Inc. It consisted of an electrical resistance element encased in Kapton. The heater was rated at $10 \mathrm{ohms}$. This small value was chosen so the heat flux produced by the heater could be more easily regulated if it became necessary to do so. The electrical element was not uniform over the entire area. It was a long wire-like element which had been sculpted to fit within a certain area. However, it approximated a uniform heat flux, 


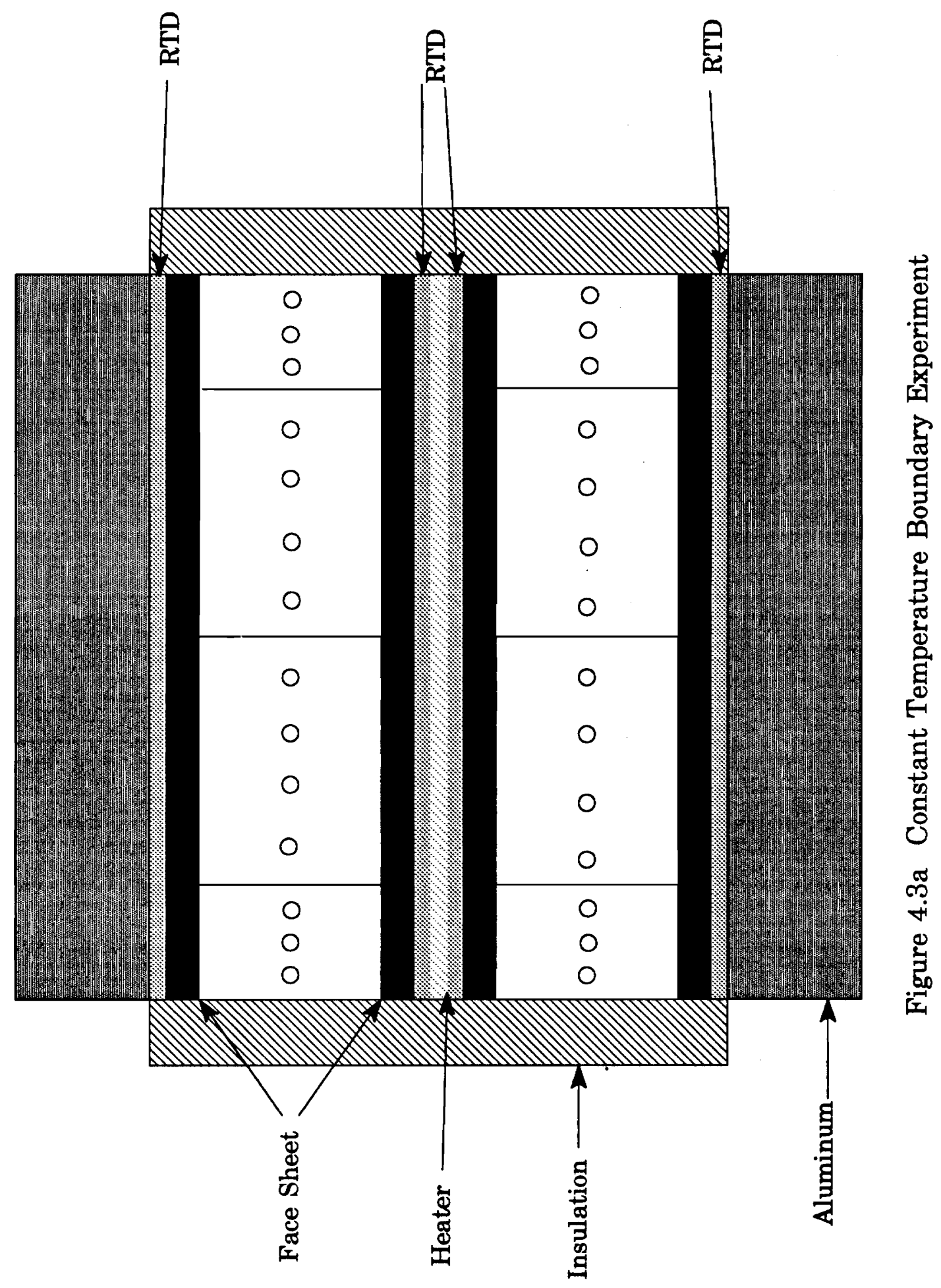




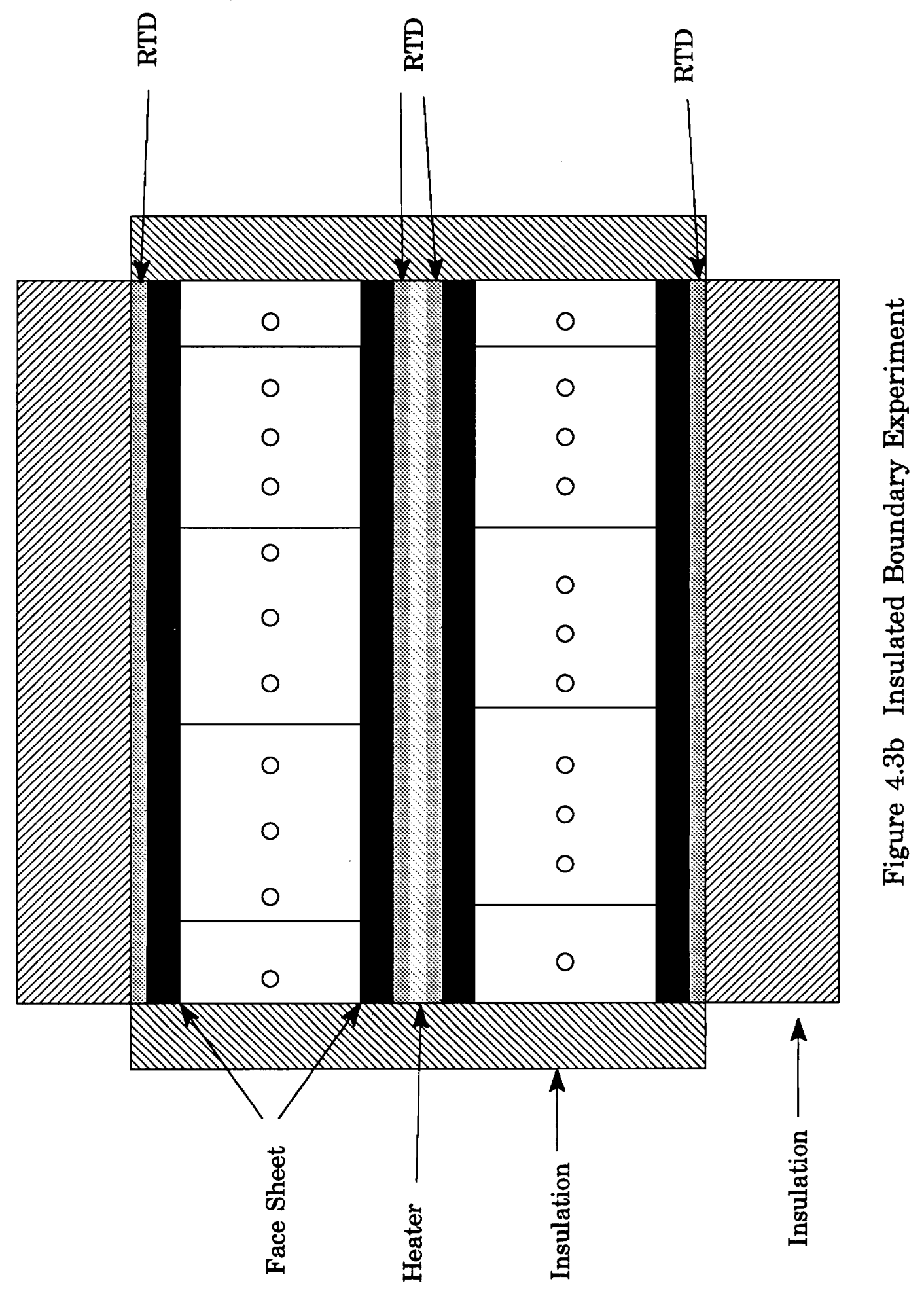


due to the low thermal resistance of the face sheets of the samples. The lead wires were externally attached to the heater to prevent added contact resistance at the heater surface. The voltage and current drawn by the heater was continuously measured. This was done to obtain an accurate reading of power consumption which might change over time due to variations in input voltage and resistance change as a result of heating. The heater was powered by a Hewlett - Packard DC power supply capable of producing over 6 watts. This was sufficient to make the heater put out about $350 \mathrm{~W} / \mathrm{m}^{2}$. This apparatus and the rest of the equipment are shown in Figure 4.4.

The oven used in this experiment was a 7500 watt Series 3710A made by Applied Test Systems, Inc. The purpose of the oven was to provide an environment where high temperature experiments could be conducted. The oven was capable of reaching temperatures well over $811 \mathrm{~K}(1000 \mathrm{~F})$. The maximum temperature that was attained in any experiment was $495 \mathrm{~K}(430 \mathrm{~F})$. No higher temperature was used for fear of destroying the Kapton coating of the heater and RTD's.

The data acquisition system used in this experiment consisted of two parts. The software used was Autonet 4.2 by Imagination Systems, Inc. The hardware consisted of a NEFF 470 Data Acquisition Unit with a NEFF 470050/90023130 16 - Channel Differential MUX card made by NEFF Instrument Corp and an ALR personal computer.

The purpose of the hardware was to bring voltage signals into the data acquisition program. There were six signals being recorded: four RTD measurements, voltage to the heater, and current drawn by the heater. The RTD voltages were read directly from the bridge circuit by the NEFF unit. The dynamic range of the system was always set to provide the highest possible resolution. Heater voltage was read from a Keithley digital multimeter. Current was read from a similar multimeter with the output voltage 


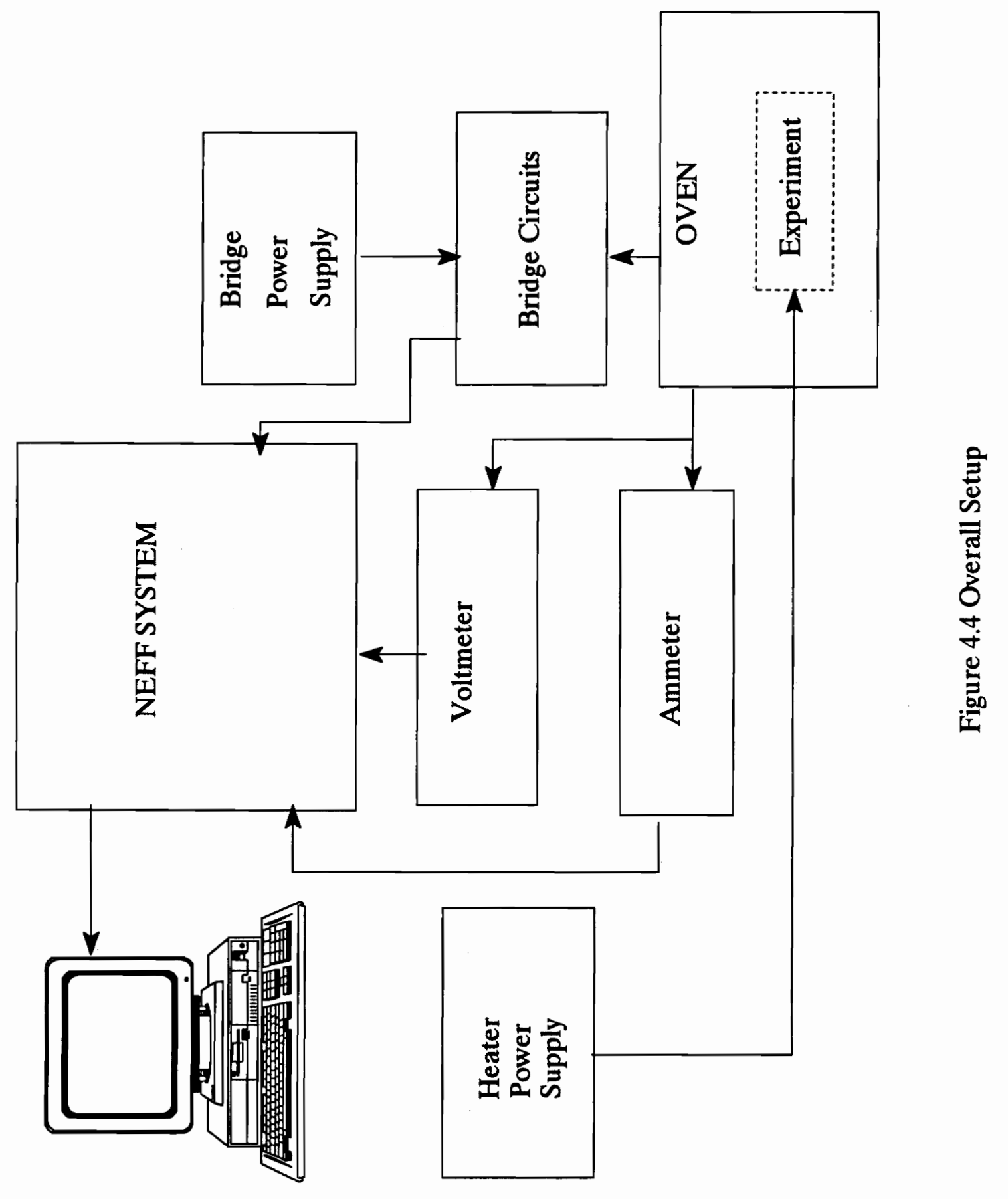


proportional to the current $(1$ volt $=1 \mathrm{amp})$.

The Autonet software allowed for some initial data processing. When scanning was started, the six voltages plus the time of the experiment were recorded. Heater current and voltage were converted into heat flux by:

$$
q^{\prime \prime}=E I / 2 A
$$

where $\mathrm{q}^{\prime \prime}$ is the heat flux, $\mathrm{E}$ is the voltage, $\mathrm{I}$ is the current, and $\mathrm{A}$ is the surface area of one side of the heater. The RTD voltages were also converted into temperature by Eq. (4.4).

\subsubsection{Temperature Sensors}

As previously stated, it was necessary to measure the temperature at both surfaces of the honeycomb sample as it was being heated. There are several devices which can be used to measure temperature. Thermocouples are probably the most popular. They were not chosen for this study for several reasons. First, there would be grounding problems due to the metallic nature of the honeycomb structure. The output from thermocouples is also noisy. Thermocouples measure temperatures at a point. Since a one dimensional model is being used, point measurements were not necessary. If a multi-dimensional model were used then point measurements would be necessary. However it would be nearly impossible to accurately locate thermocouples or anything else on the face sheet with respect to the core components of the structure. In addition, the high conductivity of the face sheet would destroy any thermal gradients which might 
result from the core. These are perhaps the most compelling reasons to use a one dimensional heat transfer model.

The temperature sensor that was chosen was the RTD. The RTD, or Resistance Temperature Detector, is a resistance thermometer which responds positively to temperature. Four RTDs were used as previously stated. They contain platinum elements which have nominal resistance of $2000 \Omega$ at $70 \mathrm{~F}$. They were made by Minco Products, Inc. They are the same size as the face sheet of the samples. Two-wire leads were used due to the fact that the lead wire length was relatively short.

The resistance of each RTD was calibrated with respect to absolute temperature. This was done by placing each RTD in oil in an oven and the resistance recorded at $50^{\circ} \mathrm{F}$ increments over the range of operation. The calibration was performed by Wyle Laboratories, Inc. It was necessary to know resistance as a function of absolute temperature due to the fact that radiation was being modelled.

A third order polynomial was used to regress the temperature data as a function of resistance:

$$
T=f(R)=a+b^{*} R+c^{*} R^{2}+d^{*} R^{3}
$$

This type of curve produced a correlation coefficient of 0.999. A typical curve is shown in Figure 4.5. It is nearly linear.

Instrumentation was necessary to determine the resistance of the RTD at any temperature. The setup is shown in Figure 4.6. The constant-current bridge was chosen as the instrumentation circuit. The constant-current bridge was chosen over the constant-voltage bridge because its output is more linear. The three inactive arms of each bridge were completed with $2000 \Omega \pm 0.005 \%$ resistors. It could then be assumed that 


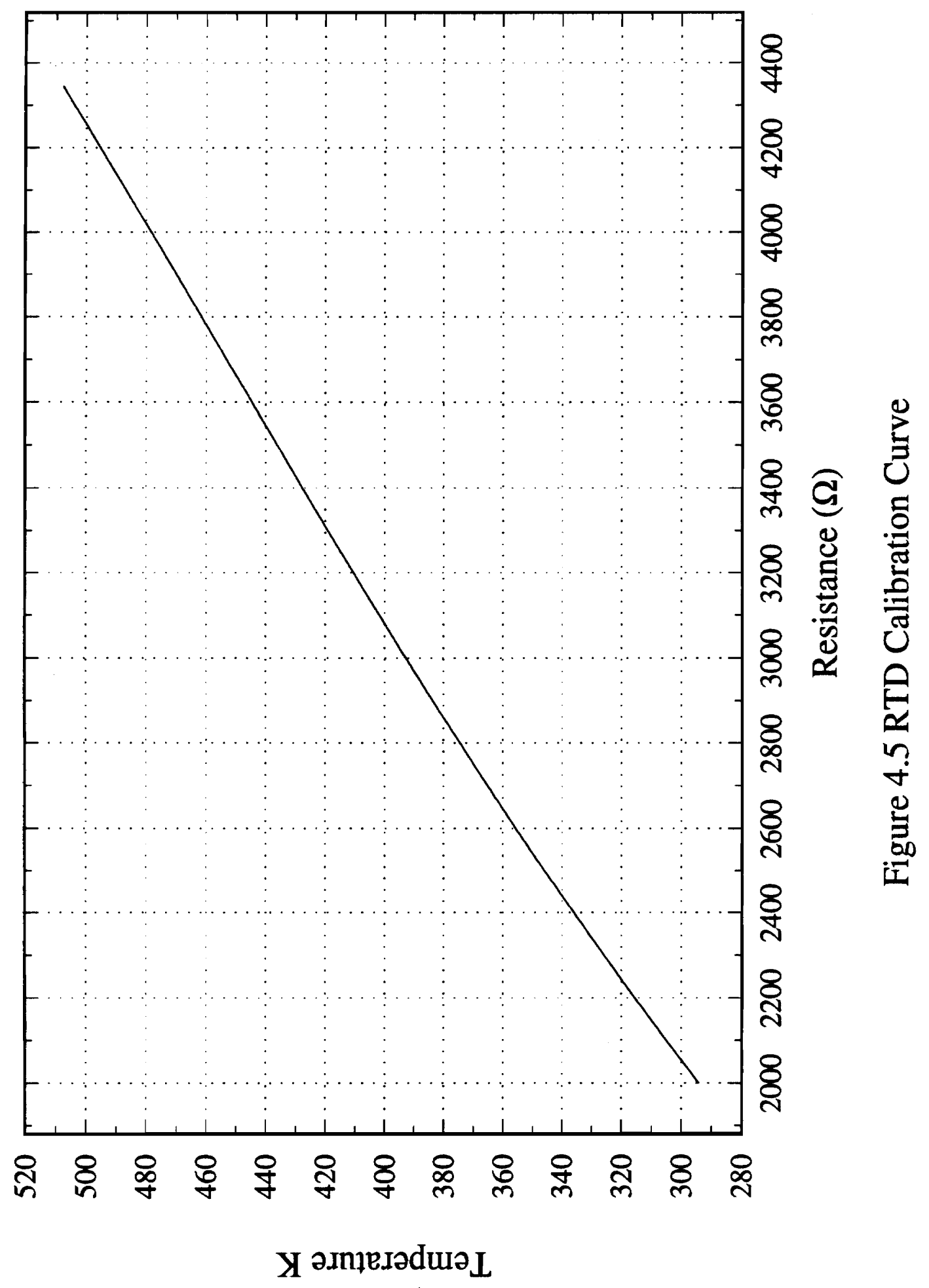




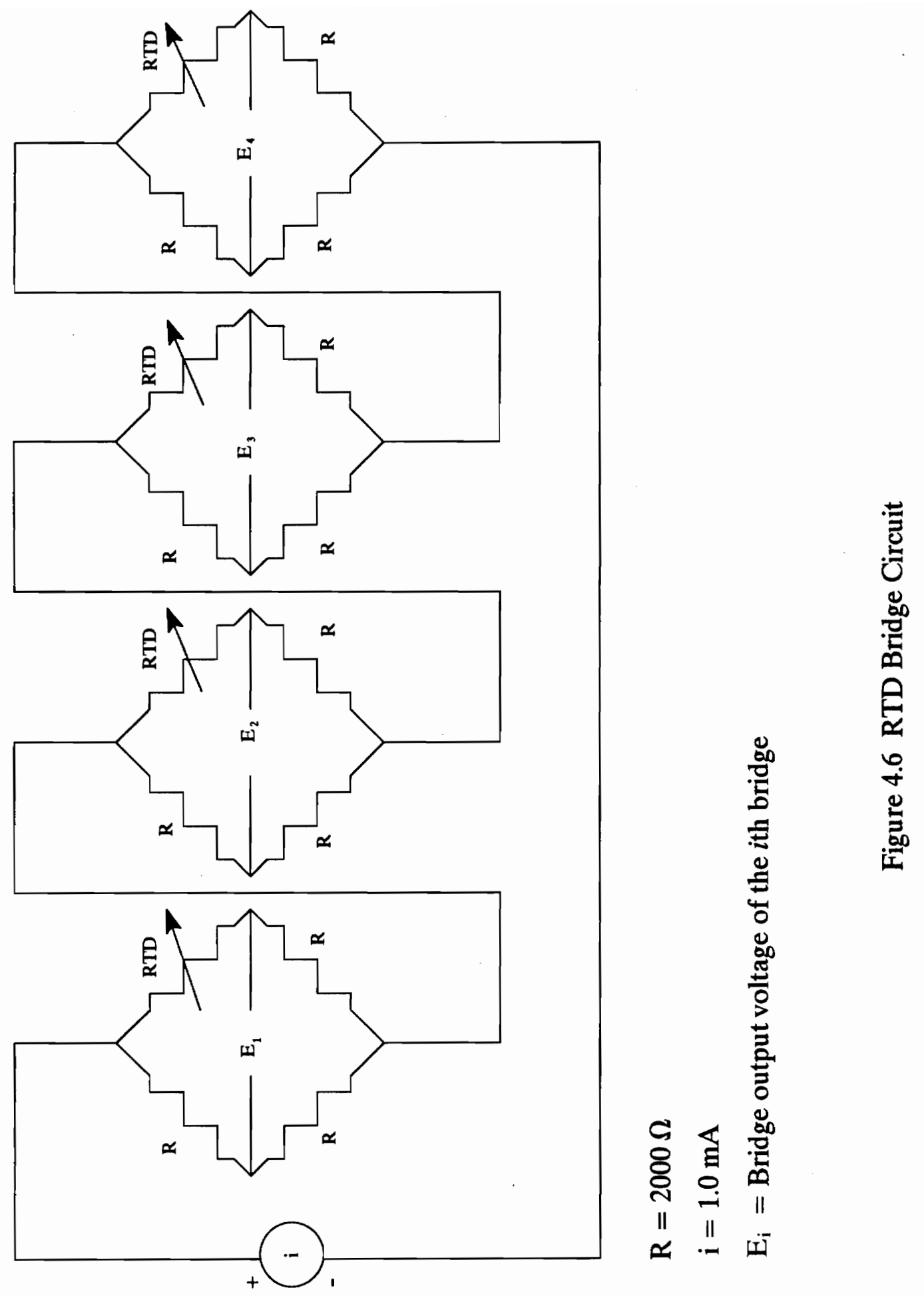


the bridges were all balanced at $295 \mathrm{~K}(70 \mathrm{~F})$, eliminating the need for a balancing potentiometer and allowing for the direct calculation of absolute temperature from a resistance change. The four bridges were placed in series and powered by a $1.0 \mathrm{~mA}$ current source. The resistance of the bridge is then:

$$
R_{R T D}=\frac{3 R E_{B}+I R^{2}}{I R-E_{B}}
$$

Since $R=2000 \Omega$ and $I=1.0 \mathrm{~mA}$

$$
R_{R T D}=\frac{6000 E_{B}+4000}{2-E_{B}}
$$

where $E_{B}$ is voltage output across the two arms for each individual bridge.

Note that the current used by the bridge circuit will cause some heating in the RTDs, which could be a source of error. The power dissipated by each RTD is equal to the product of its resistance and the square of the current in the circuit:

$$
P=I^{2} R
$$

Since the current is $0.001 \mathrm{~A}$ and the resistance is $2000 \Omega$, the power dissipated by each RTD is $0.002 \mathrm{~W}$. This heating is assumed to act uniformly over the surface of each RTD. With an area of $0.009677 \mathrm{~m}^{2}$, each RTD will see the effect of only $0.207 \mathrm{~W} / \mathrm{m}^{2}$ due to internal heating. Since $350 \mathrm{~W} / \mathrm{m}^{2}$ is applied for each test, the effect of internal heating is negligible. 


\subsection{Thermal Image Analysis}

Honeycomb-core sandwich structures are inherently three dimensional. From a thermal standpoint they may be if the boundary condition on each surface is kept uniform. The purpose of thermal imagery was to determine where the major thermal gradients exist in the structure. If the in-plane gradients are small compared to the gradient through the thickness, then the heat transfer can be modelled as one dimensional.

The samples were prepared in a manner similar to the way in which later experiments would be run (see Section 4.3). The only exception was that one side of each samples was left exposed. The exposed side was painted black in order to have the highest possible emissivity. The sample was then heated to steady-state and viewed with an Agema Thermovision 880 thermal camera. The camera gave an infrared image of the sample.

Figure 4.7 shows the resulting image. The camera was aimed at an angle which permitted a view of both a face sheet and the core. The bottom third of the figure shows the face sheet. A camera's view of the structure is shown in Figure 4.8. There is a small temperature gradient from the edges to the center. However, it is relatively small in magnitude. This confirms the fact the thermal conductivity of the aluminum/boron matrix face sheet is sufficiently high enough to eliminate any thermal gradients in the face sheet.

Near the left of the image the outline of one section of the core can be seen. It is slightly cooler than surrounding regions. The core begins where the major thermal gradient starts and covers the top two-thirds of the figure. The core is slightly warmer 


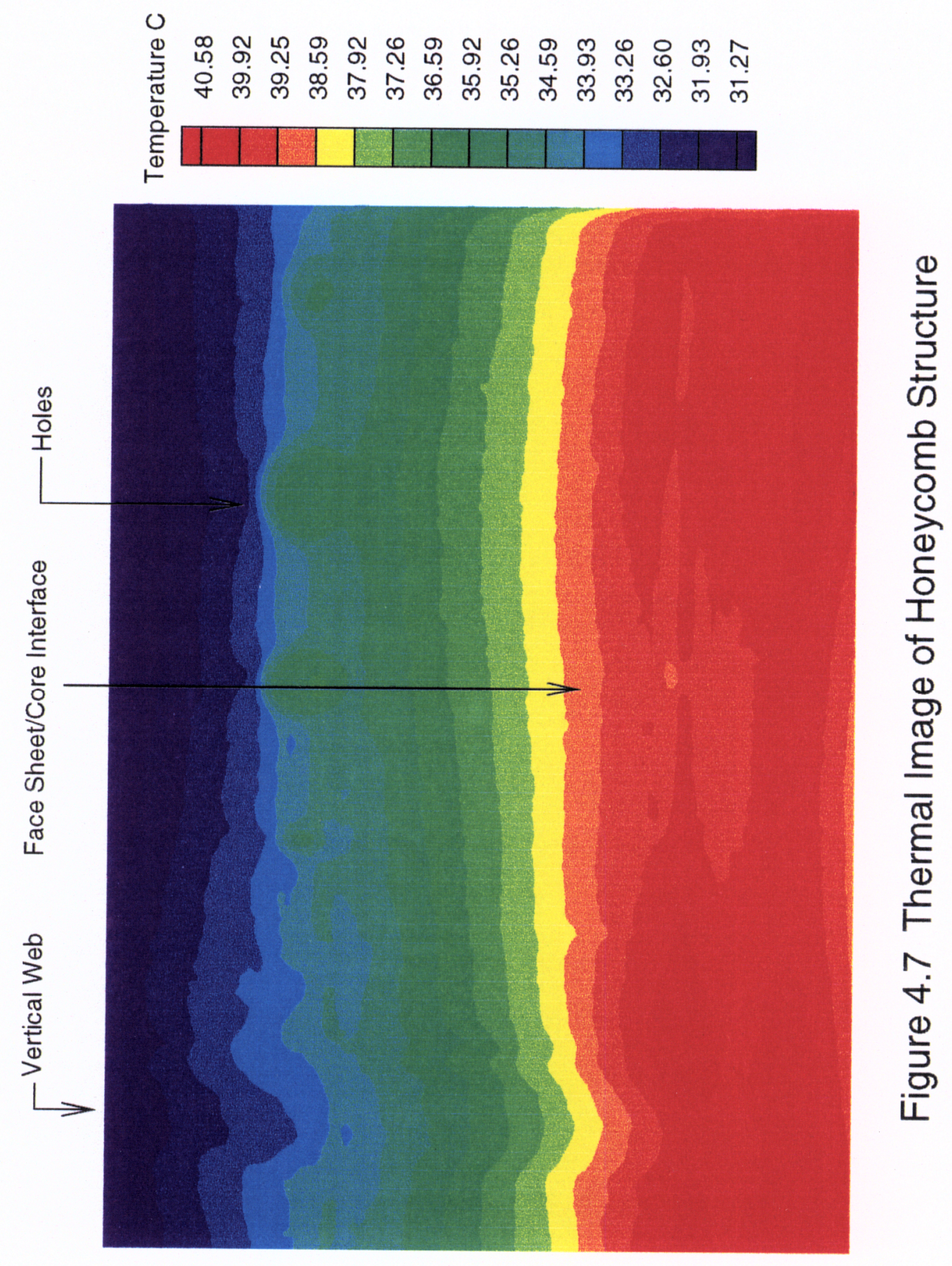




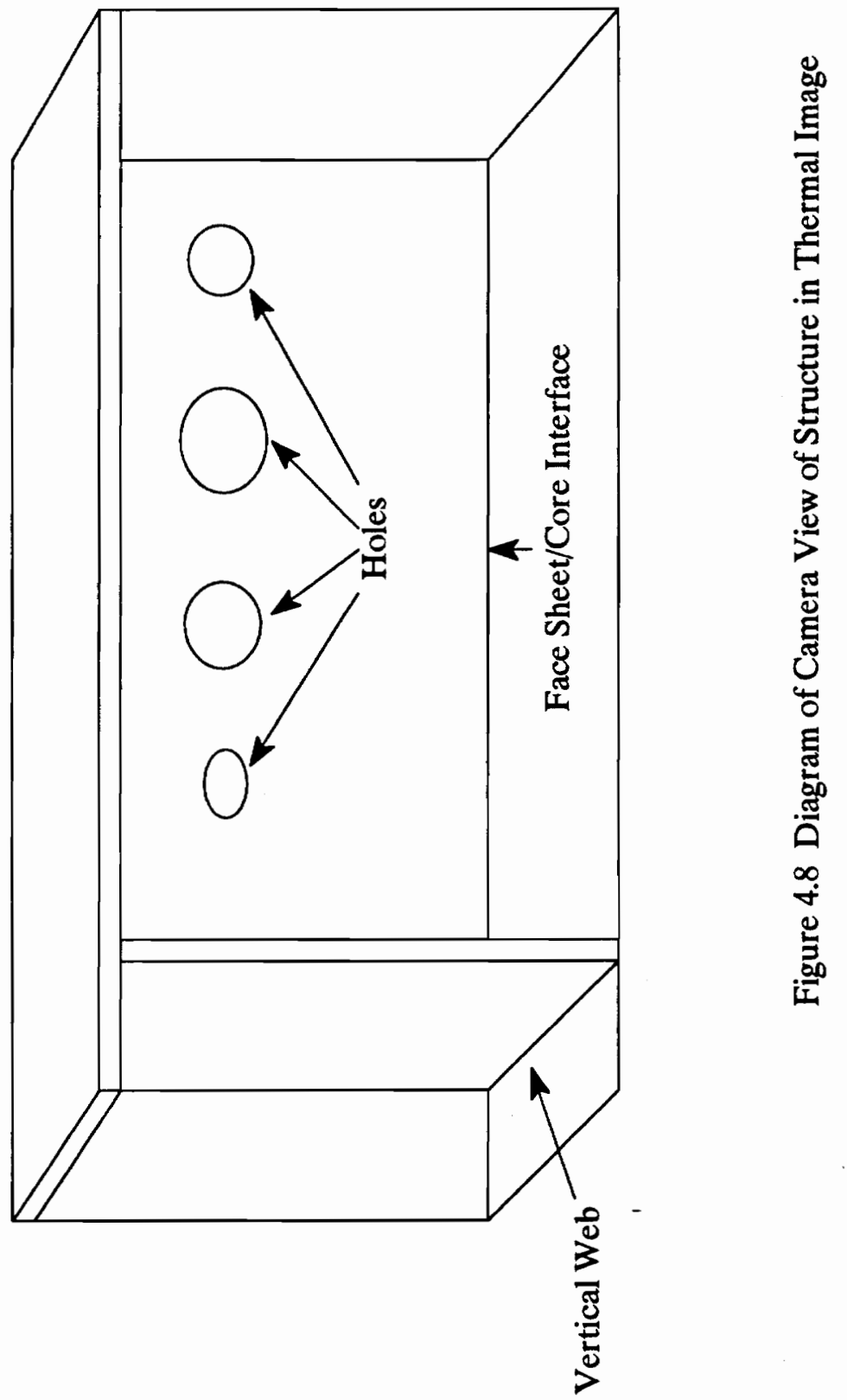


in the center, away from the web intersections. Notice that there are no strong two dimensional effects. The row of holes can also be seen. They show up as warm circles due to the fact that the camera is looking through the holes to a warmer spot. The welds are not noticeable.

The fabrication process does not produce any effects which are thermally significant. Nor are there any signs of contact resistance at the RTD interface. It is also clear that the thermal gradient through the thickness is much greater than any in-plane gradient in the face sheets or the core itself. Therefore, a one dimensional model should be adequate.

\subsection{Description of Experiments}

It was desired to investigate the thermal properties of the structure over a range of temperatures. Room temperature, $295 \mathrm{~K}(70 \mathrm{~F})$, was chosen to be the lowest temperature investigated, while $495 \mathrm{~K}(430 \mathrm{~F})$ was chosen to be the highest starting temperature. The kapton coating on the RTDs and heater will carbonize and disintegrate near $573 \mathrm{~K}(572 \mathrm{~F})$. Maximum test temperatures were therefore held below this point. Experiments were conducted from $295 \mathrm{~K}(70 \mathrm{~F})$ to $495 \mathrm{~K}(430 \mathrm{~F})$ in increments of $50 \mathrm{~K}$ (90 F). Two boundary conditions, constant temperature and insulated, were investigated at each temperature. In addition, each of the aforementioned experiments were duplicated twice. The following procedure was preformed to conduct an experiment: 
1. Begin with an aluminum block (constant temperature experiment) or insulation (insulated experiment). If the aluminum was used, then a thin layer of thermal joint compound was spread on it. The experiment was built up as previously described.

2. Insulation was placed around the edges of the sample. Thin wire was tied in two places and then tightened in order to hold the insulation in place.

3. The apparatus was placed in the oven.

4. RTD and heater leads were placed through the portal in the oven and insulation stuffed around them.

5. The RTD leads were connected to the bridge circuit, while the heater wires and DC power supply leads were connected to each other, as well as the voltmeter.

6. The oven control was programmed to perform a temperature soak at the desired temperature. The heater was also turned on to heat the sample. This was the only effective means of heating the sample with the insulated boundary condition.

7. When the desired starting temperature was reached the heater was turned off and the sample allowed to reach steady state. 
8. The data acquisition program was then initiated and the heater turned on. Heating time was kept by a stopwatch.

9. The heater was turned off when the desired heating time had been reached. The data acquisition program was stopped after the total experiment time had been reached.

\subsection{Experimental Data}

The purpose of this section is to describe the data which was collected and its incorporation into the finite element program. Figure 4.9 shows a typical data set obtained from the constant temperature configuration. Two sets of data were obtained from each experiment because two samples were used. The temperature of the heated surface approaches steady state then drops back to near its initial temperature. The boundary temperature rises slightly, indicating that the aluminum heat sink is not ideal. Because this boundary is not truly constant, it will now be referred to as the specified temperature boundary. The data set shown in Figure 4.9 has a slight offset, but when this is removed the profiles look exactly the same. This indicates that there is no significant convection, forced or natural, occurring.

A typical data set for the insulated case is shown in Figure 4.10. The temperature of the heated surface rises linearly then drops off as the heater is turned off. The non-heated surface temperature rises in a slow linear fashion, then becomes almost constant and finally begins to drop off. This is not what is expected. The temperature profile should continually increase as the sample goes to equilibrium. 


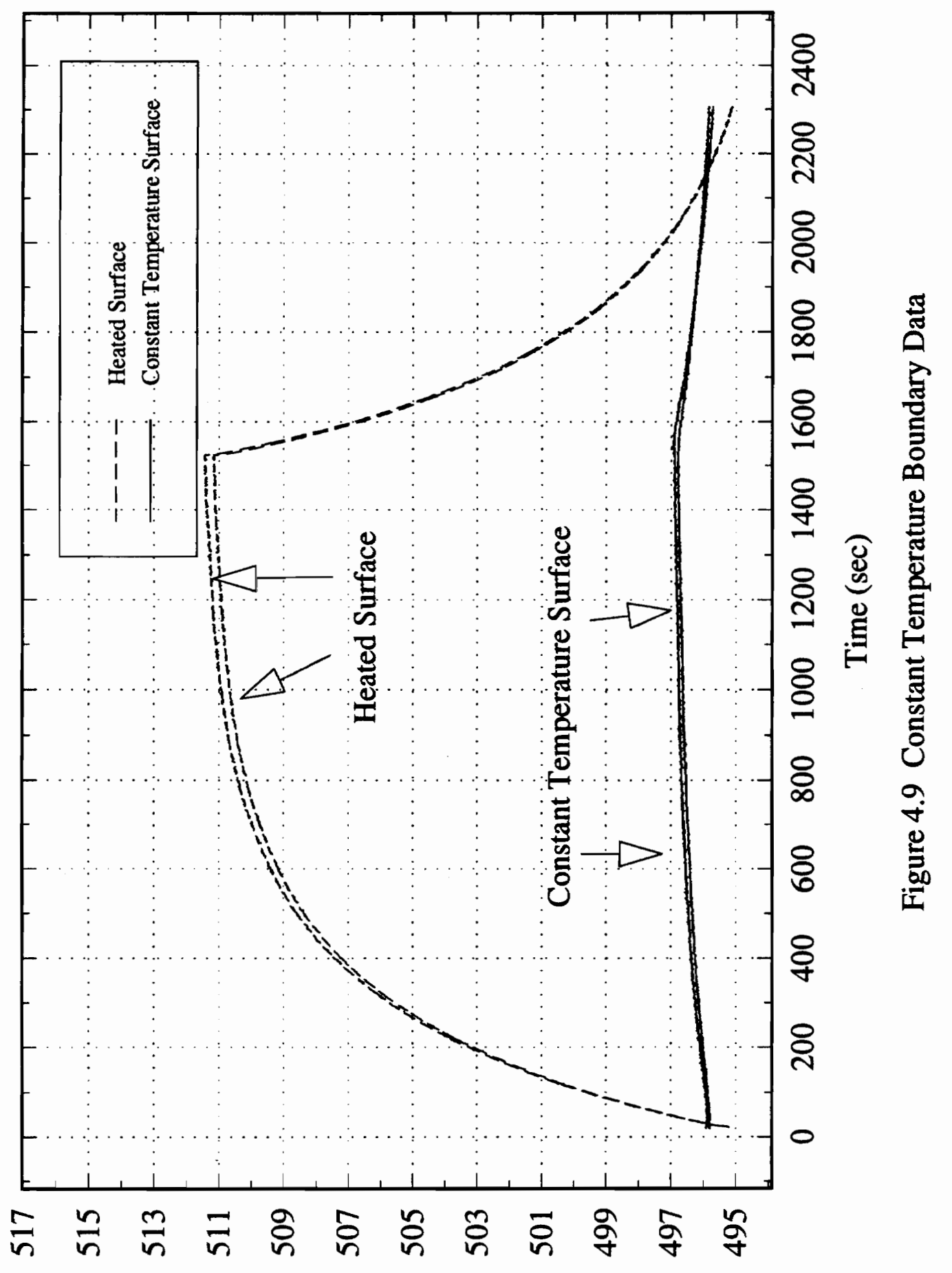

(X) əInฺ๋๖əduə L 


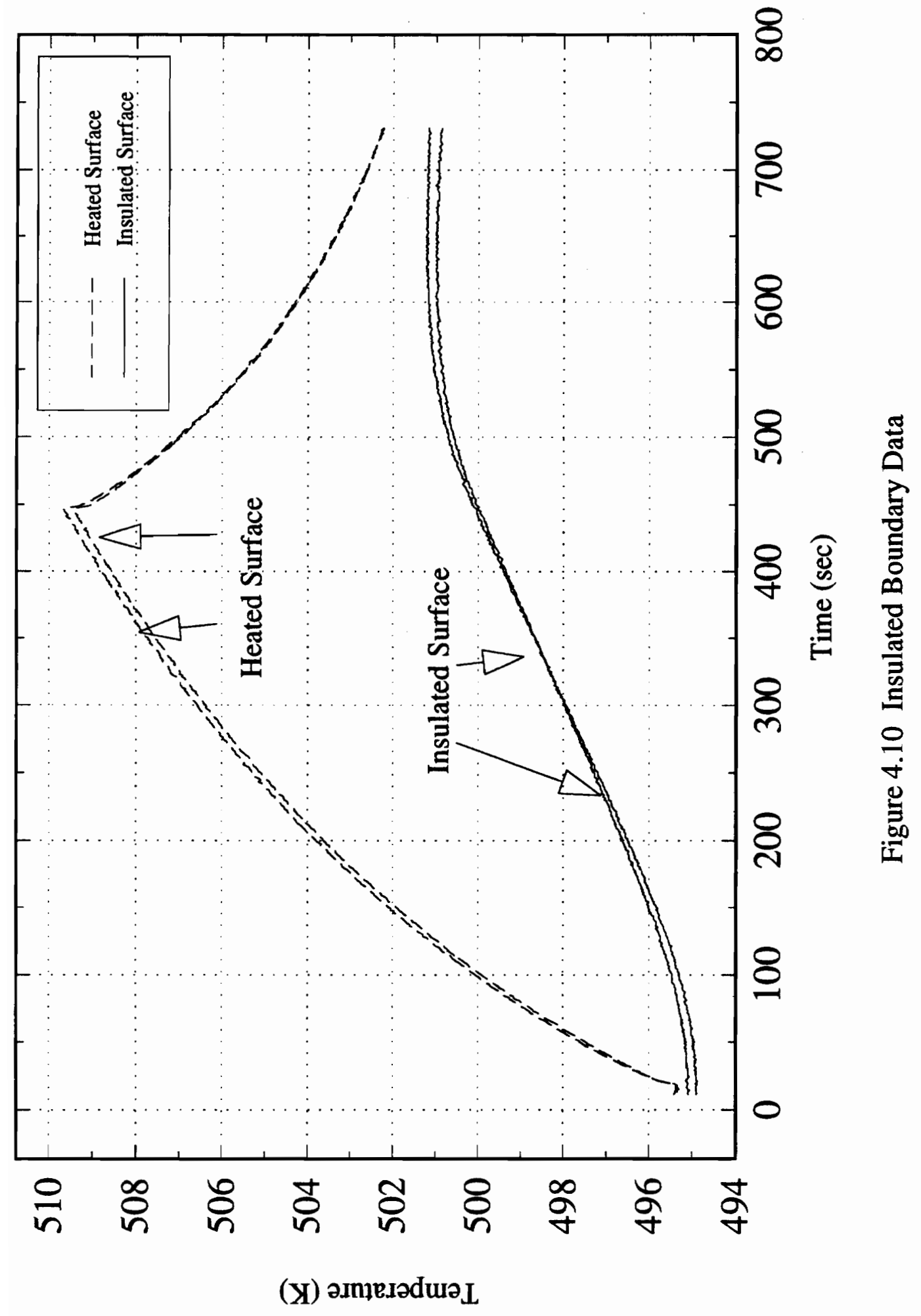


The problem is due to the non-ideal nature of the insulation. It acts as a heat sink due to the length of the experiment. It is also obvious that the insulation did not have the same uniform temperature distribution that the sample had when the experiment was started. Furthermore, it is almost impossible to predict the temperature distribution in the insulation after the first experiment due to the number of tests which were run in a single day. Each time a new experiment was run, the setup was heated to the next highest starting temperature. This was accomplished by using both the oven and the resistance heater. This non-uniform heating from two sides gave the insulation a nonlinear and unknown temperature distribution. The amount of time required for the insulation to come to equilibrium makes use of the insulated boundary condition somewhat impractical. It also precludes the use of data at that surface in the objective function of the estimation procedure. The data could be used to specify a temperature boundary condition as in the constant temperature case. 


\section{CHAPTER 5}

\section{Implementation of Finite Element Program}

The majority of the computer programming in this study involved use of the finite element program EAL (Engineering Analysis Language, Whetstone, 1983). This chapter describes how this code was used to implement the parameter estimation, experimental design, and confidence interval calculations described in Chapter 3.

\subsection{Utilization of EAL}

EAL is a finite element program which is commanded through an interpretive language. This language is used to execute various processors which serve to build an elemental model and perform calculations based on it. In addition, it has some of the capabilities of a general programming language such as algebraic manipulation and logical structures. Data can be stored in tables which have up to three dimensions, or in single valued registers.

The sequence of commands used to write an EAL program is called a runstream. A diagram of a typical EAL runstream is shown in Figure 5.1. The first processor to be 
Step 1:

TAB $\longrightarrow$ Joint Locations

Step 2:

AUS $\quad \longrightarrow \quad \begin{aligned} & \text { Table Definition - } \\ & \text { Material Properties }\end{aligned}$

Section Properties

Step 3:

$\longrightarrow$ ELD $\longrightarrow$ Element Definition

Step 4:

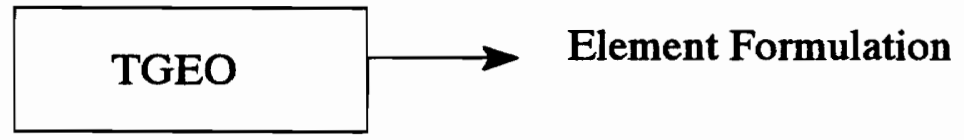

Step 5:

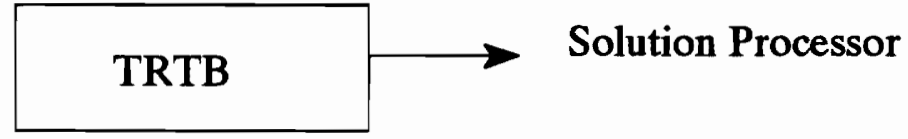

Step 6:

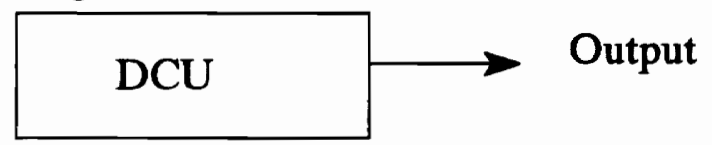

Figure 5.1 Finite Element Formulation Using EAL 
executed when beginning a model is TAB. This processor defines joint (node) locations and sets the coordinate axes. Processor AUS is then executed to construct needed tables. Tables must be specified which contain pertinent material, section, and excitation properties. Processor ELD is used to define element types on the grid. Processor TGEO constructs the geometry according to the defined element types. Different types of elements corresponding to heat transfer, fluid mechanics, or deformation can be placed on the same nodes, allowing for combined thermal/mechanical analysis. The next step is to execute a solution processor(s) corresponding to the type of analysis being done. In this case, processor TRTB calculates temperatures with an implicit scheme. Processor DCU is then executed to view output files.

A sample runstream is shown in Table 5.1. This program is used calculate the temperature response of the insulated boundary case to transient heating. It follows the diagram shown in Figure 5.1.

\subsection{Minimization Procedure}

The minimization procedure used in this study involved three distinct compo-

nents: the finite element code, an optimization routine, and experimental data. EAL provided the structure for the entire algorithm. This section describes how the experimental data were incorporated into the program, how temperatures were calculated from the finite element code, and how the optimization was performed. 
Table 5.1 Sample EAL Runstream

*XQT TAB

-This section defines the joint locations

START 11

(11 nodes with a length of .024081)

JLOC: 10.0 .0 .0250810 .0 .111

${ }^{*} X Q T$ AUS

4 Table definition

TABLE(NI=9,NJ=2): COND PROP 1: I = 123456 4Conduction properties

$\mathrm{J}=1: 295.4420 .558 .27 .57 .57 .5$ of the core

$\mathrm{J}=2: 533.4420 .619 .510 .710 .710 .7$

TABLE(NI=1,NJ=3): K AREA: J=1:

$\mathrm{J}=2: \quad .001419$

J=3: $\quad 2.14 \mathrm{E}-6$

3.52E-5 $\triangleleft$ Core Section areas

TABLE(NI=9,NJ=1): COND PROP 2: I=3 45 6: J=1: 0. 0.0 .0 .

TABLE(NI=1,NJ=1): SOUR K21 2: BLOCK 1: J=1: 350 .

BLOCK 2: J=1: 350 .

Heat flux magnitude

BLOCK 3: $\mathrm{J}=1: 0$.

and duration

BLOCK 4: J=1: 0 .

TABLE(NI=1,NJ=4): SOUR TIME: J=1: 0 .

$\mathrm{J}=2: 427$.

$\mathrm{J}=3: 427.1$

$\mathrm{J}=4: 700$.

TABLE(NI=9,NJ=1): COND PROP 3: I=2 345 6: J=1: 1. 2567700. 0. 0. 0. 4Face sheet

TABLE(NI=4,NJ=1): RADI PROP 1: I=2 3 4: J=1: .678 .678 .322

TABLE(NI=1,NJ=2): $\mathrm{R} \quad$ CIRC: J=1: .001384 «Radiation Properties $\mathrm{J}=2: \quad .15378$

TABLE(NI=1,NJ=1): SB CONS: J=1: 5.6697E-8

${ }^{*}$ XQT ELD

RESET NUTED $=1$

K21

GROUP 1

12120

GROUP 2

NSECT $=2$

$\mathrm{NMAT}=2$

11

GROUP 3

NSECT $=3$

$\mathrm{NMAT}=3$

1 $1: 1111$

R21

GROUP 1 $\checkmark$ Element Definition

$\triangleleft$ Conduction Elements $\downarrow$ Radiation Elements 
Table 5.1 - Continued

NSECT $=1$

11

GROUP 2

$\mathrm{NSECT}=2$

12110

GROUP 3

NSECT $=1$

1111

*XQT TGEO

*XQT TRTB

-Element Formulation

RESET PRINT $=0$

RESET T1 $=0$.

RESET T2 $=700$.

RESET DT $=10$.

-Transient Implicit Temperature Analyzer

$\checkmark$ Reset commands set length of computation, calculation

tolerances, initial conditions, times load vectors are

computed, etc.

RESET BETA $=0.5$

RESET NFACS $=5$

RESET MXNDT $=100000$

RESET RNITER $=10$

RESET KTI=1

KTIME $=10$.

$\mathrm{TEMP}=495.5$

TSAVE $=10$.

RTIME $=10$.

*XQT AUS $\triangle$ Creates a table containing temperature histories of specified nodes

DEFINE $A=1$ TRAN TEMP 11170

DEFINE $B=1$ TRAN TIME 11

TABLE(NI=3,NJ=70): ENDN TEMP

TRANSFER(SOURCE $=\mathrm{A}, \mathrm{SBASE}=0, \mathrm{ILIM}=1, \mathrm{DBASE}=0, \mathrm{DSKIP}=2, \mathrm{OPER}=\mathrm{XSUM}$ )

TRANSFER(SOURCE=A,SBASE $=11, \mathrm{ILIM}=1, \mathrm{DBASE}=1, \mathrm{DSKIP}=2, \mathrm{OPER}=\mathrm{XSUM}$ )

TRANSFER(SOURCE=B,SBASE=0,ILIM=1,JLIM=70,DBASE=2,DSKIP=2,OPER=XSUM)

*XQT DCU

PRINT 1 ENDN TEMP

${ }^{*} X Q T$ EXIT 


\subsubsection{Experimental Data}

The temperature histories which were measured by the RTD's were pre-processed before being incorporated into the estimation program. Since the experiment was designed to provide symmetrical heating in the two samples, and the temperature measurements at corresponding locations in the samples were nearly identical, the data taken from corresponding RTD's at the heated surface and outer boundary were averaged together. Any initial offsets were removed prior to averaging. All data recorded prior to just before the first heat flux measurement were used to determine the offset and then deleted. The first data points of each sensor were all associated with a time of 0.0 .

This data was used to create a data file which contained the time, temperature at the heated surface, temperature at the non-heated surface, and heat flux. This file was read directly into EAL as a table. The first temperature measurements $(t=0)$ at each surface were used to obtain a linear temperature distribution through the sample which served as the initial conditions for the model.

\subsubsection{Minimization Algorithm}

The minimization routine used in this study follows the flow chart shown in Figure 5.2. The actual runstream can be found in Appendix B. The analytical model is set up as described in the previous section. Processor TRTB calculates the transient temperature response of the model. Its calculations are based on material and section properties of the sample. This includes the unit cell dimensions and thermal properties of the sample. Note that the parameters being estimated are left as variables and 


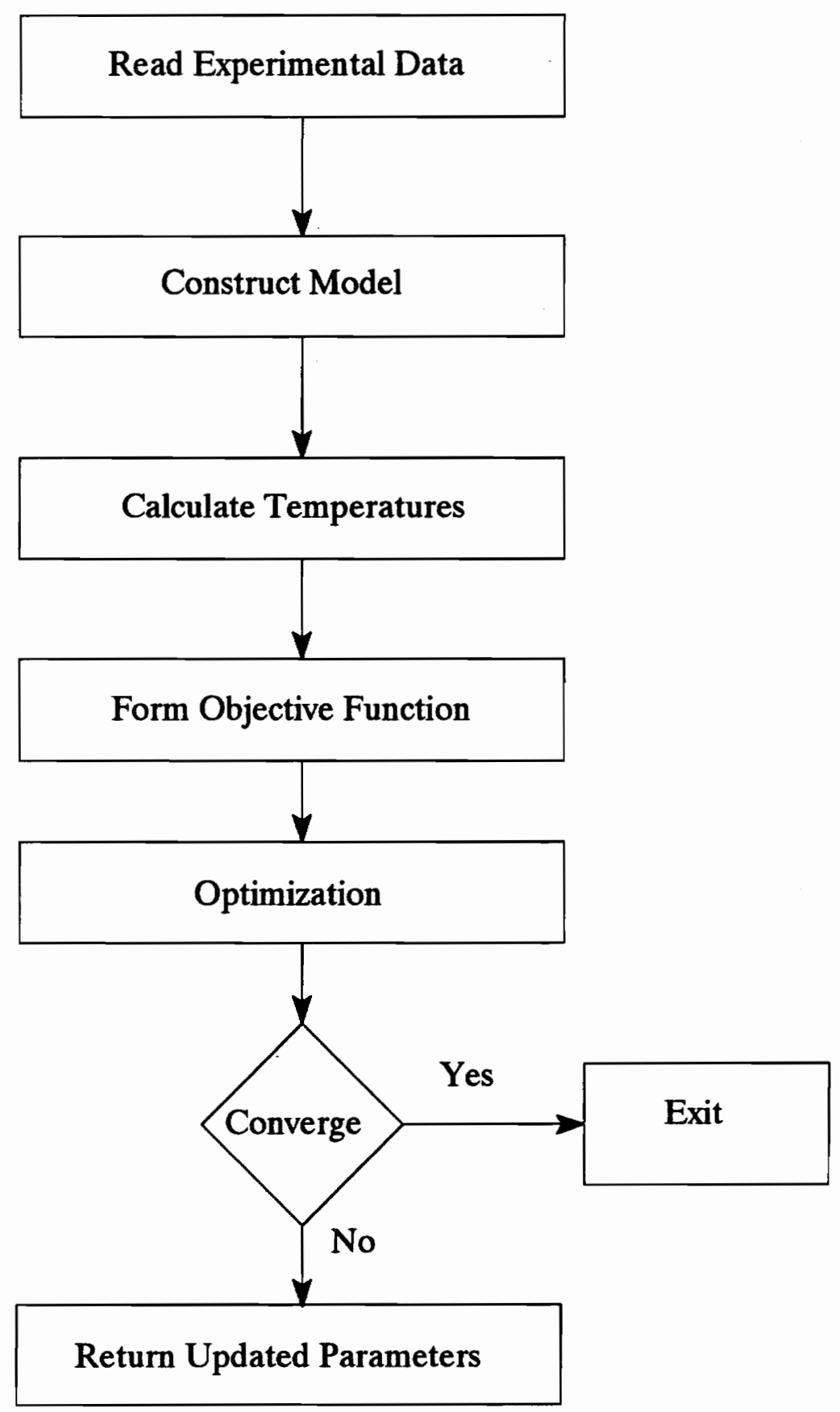

Figure 5.2 Estimation Program Flow Chart 
changed from iteration to iteration. If data from two sensors are used in the estimation procedure, then the temperatures at the nodes corresponding to each face sheet are stored in a separate table. If only one sensor is used, then only the temperatures corresponding to the node at the heated surface are placed in this table. These temperatures now form a new data set the same length as the experimental data set. In the case of two sensors, the two temperature histories are concatenated, both for the calculated and experimental data.

The objective function is formed by first subtracting the experimental data set from the corresponding calculated temperatures, squaring the difference, and summing all the squared differences. It is minimized by the optimization processor pursuant to a data set of constraints. If the optimization processor determines that convergence has occurred, the program is terminated. If convergence has not occurred, then a new set of parameters is returned to the program and a new set of temperatures is calculated.

Constraints for each parameter were chosen to prevent numerical difficulties in calculating temperatures. The parameter which was the most unstable was the emissivity. Outer bounds of 0.4 and 1.0 were placed on this parameter. It is physically impossible for $\varepsilon$ to be greater than 1.0. Values less than 0.4 caused convergence problems in EAL's radiation load vector computation. This was justified since most references list the emissivity of titanium as greater than 0.5 . The other two parameters were initially scaled to 1.0 and bound to within a factor of 10 . The conduction area was scaled by a factor of $3.5 \times 10^{-5} \mathrm{~m}^{2}$ which was derived from an average of actual measurements. The volumetric capacitance of the face sheets were scaled by a factor of $2.7 \times 10^{6} \mathrm{~J} / \mathrm{m}^{3} \mathrm{~K}$ (Touloukian et al., 1970). 


\subsection{Experimental Design}

EAL was also used for the experimental design procedure and final confidence interval calculation. This process involved building the same model as before to calculate temperatures. Temperature calculations were necessary to calculate the sensitivity coefficients. Diagonal terms of the sensitivity matrix appear in the confidence interval. Sensitivity coefficients were calculated by a forward difference method. Each parameter was perturbed by factor of 0.001 and the sensitivity calculated as shown

$$
X_{i j}(i) \approx \frac{\eta_{i}\left(b_{1}, \cdots, b_{j}+\delta b_{j}, \cdots, b_{p}\right)-\eta_{i}\left(b_{1}, \cdots, b_{j}, \cdots, b_{p}\right)}{\delta b_{j}}
$$

where $\delta$ is the perturbation. This was done for all three parameters and the resulting sensitivity coefficients stored in a table. This information was used to calculated the confidence intervals.

$$
r M \boldsymbol{X}=\boldsymbol{K} \boldsymbol{X}
$$

Another processor, STRP, was used to calculated the eigenvalues and eigenvectors of the sensitivity matrix. STRP solves the problem where, in this case, $M$ is the identity matrix, $I$, and $K$ is the sensitivity matrix, $X^{T} X$. Maximization of the minimum eigenvalue is one method of experimental design. These eigenvalues can also be multiplied together to obtain the determinant of $\boldsymbol{X}^{T} \boldsymbol{X}$, which is another means of optimization. 


\section{CHAPTER 6}

\section{Results and Discussion}

This chapter presents the analytical and experimental results obtained for the simultaneous estimation of emissivity, conduction area, and face sheet heat capacity in the honeycomb core sandwich structure. A procedure for evaluating experimental designs for both boundary conditions is given in the first section. It is based on the theory given in Section 3.4. Included are subsections on heating time and total experimental time. The next section discusses the sensitivity coefficients associated with each parameter for both boundary conditions. The results of the estimation procedures are shown in the next section. They include the specified temperature boundary, the insulated boundary with one or two sensors, and the case of data from both boundary conditions used simultaneously. A statistical analysis and comparison of the estimation procedures is then given. Due to inconsistencies in the results of different boundary conditions, it was desired to see if higher initial temperatures would produce better estimates, especially for emissivity. A numerical experiment was done for this purpose. This is shown next. The modes of heat transfer for a typical experiment are also presented. Finally, a discussion of how the estimates affect temperature profiles is given. 


\subsection{Experimental Design}

The majority of work done in the past on experimental design dealt with maximizing the determinant of $\boldsymbol{X}^{T} \boldsymbol{X}\left(\left|\boldsymbol{X}^{T} \boldsymbol{X}\right|\right)$, which is commonly known as the Dcriterion. As discussed in Chapter 3, this method may minimize some confidence intervals while actually making others larger. It was desired to find a method of experimental design which gave more attention to each confidence interval. This section examines both the determinant of $\boldsymbol{X}^{T} \boldsymbol{X}$ and the shape of each confidence interval as a function of time. The experimental conditions which were examined were the heating time and total experiment time. Other factors also discussed in brief.

\subsubsection{Heating Time}

The use of $\left|\boldsymbol{X}^{\mathrm{r}} \boldsymbol{X}\right|$ as a design procedure has been well documented. This method involves integrating the sensitivity coefficients over time. The maximum value of this function corresponds to the optimal experiment. In order to find an optimum heating time, a total experiment time is first chosen. It should be sufficiently large so that continued heating near this time adds little information about the estimated parameters. In this study, a time of 6000 seconds was chosen for the specified temperature boundary case and 1500 seconds for the insulated boundary case.

A program (EAL runstream) was written to implement this procedure. As the heating time is incremented, the sensitivity coefficients are summed over time, the $\boldsymbol{X}^{T} \boldsymbol{X}$ matrix formed, and its determinant found. Determinant values are time averaged in order to better assess the effect of added heating time. Sensitivity coefficients for the 
three parameters and the determinant were calculated as shown in Section 5.3. Initial estimates for the parameters were the same as those used in starting the estimation program $\left(\varepsilon=0.7, A=3.5 \mathrm{E}-5 \mathrm{~m}^{2}, \rho C p=2.7 \mathrm{E}+6 \mathrm{~J} / \mathrm{m}^{3} \mathrm{~K}\right)$.

The optimum heating time is chosen to be the time when the determinant of $\boldsymbol{X}^{T} \boldsymbol{X}$ reaches a maximum. Figure 6.1 shows this for the specified temperature boundary case. Note that the lower temperatures have larger determinants. Optimum heating times using this criteria are shown in Table 6.1.

The program also calculated a confidence interval for each parameter as defined by Eq. (3.25), where the sum of squares value, $S$, was replaced by a value of 1.0 . Note that the resulting values are generally meaningless quantitatively, but qualitatively their shapes give insight into the effect of heating time. They would have units of the parameters they represent, but again, this is irrelevant. Graphs of these scaled confidence intervals for the specified temperature boundary case at $295 \mathrm{~K}$ and $495 \mathrm{~K}$ are shown in Figures 6.2 and 6.3.

Table 6.1 Optimum Heating Times for the Specified Temperature Boundary Case Using the $\left|\mathbf{X}^{\mathbf{T}} \mathbf{X}\right|$ Criteria

\begin{tabular}{||c||c||}
\hline Initial Temperature & Heating Time (sec) \\
\hline \hline $295 \mathrm{~K}$ & 4560 \\
\hline $345 \mathrm{~K}$ & 4750 \\
\hline $395 \mathrm{~K}$ & 2000 \\
\hline $445 \mathrm{~K}$ & 1690 \\
\hline $495 \mathrm{~K}$ & 1500 \\
\hline
\end{tabular}




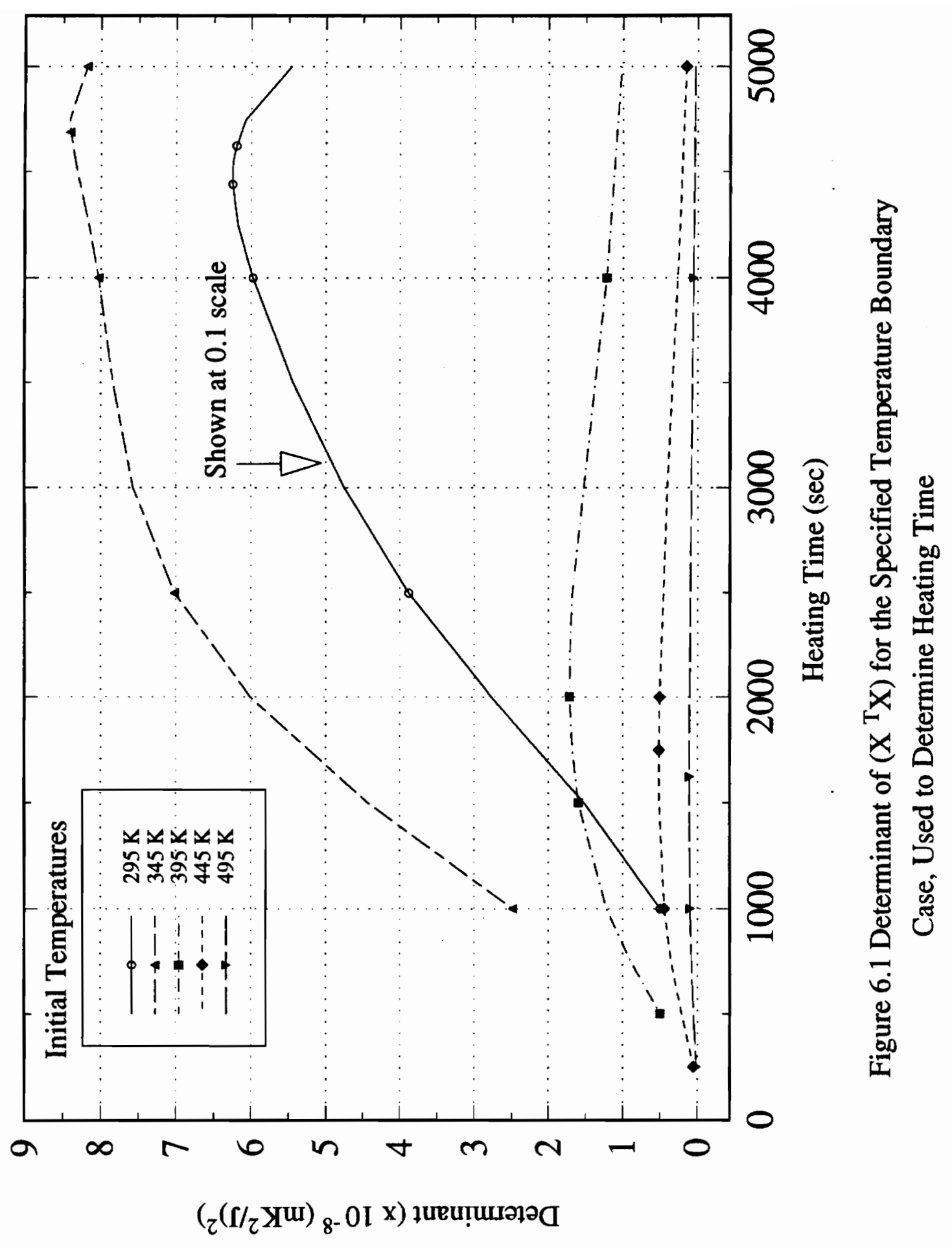




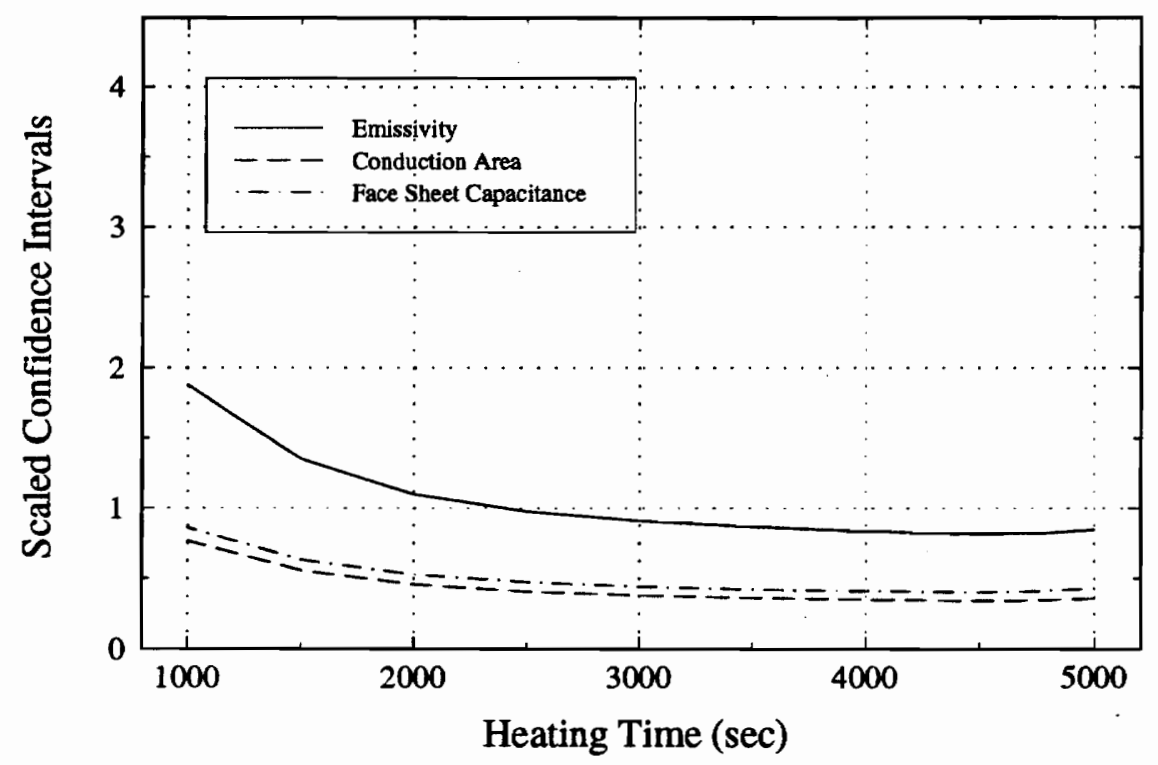

Figure 6.2 Scaled Confidence Intervals at $295 \mathrm{~K}$ for the Specified Temperature Boundary Case, Used to Determine Heating Time

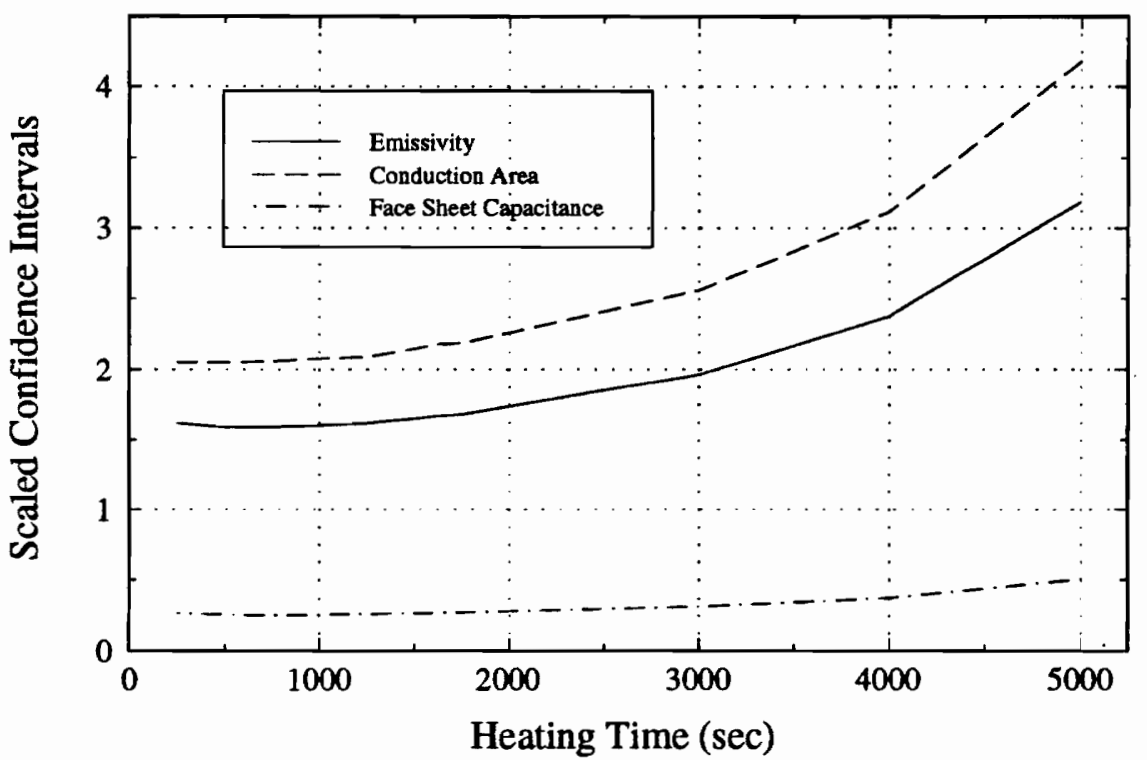

Figure 6.3 Scaled Confidence Intervals at $495 \mathrm{~K}$ for the Specified Temperature Boundary Case, Used to Determine Heating Time 
Figure 6.2 shows that emissivity has the largest confidence interval at $295 \mathrm{~K}$. Its minimum occurs near the optimum point given by the $\left|\boldsymbol{X}^{T} \boldsymbol{X}\right|$ criteria. However, it is clear that its value will not appreciably change over the range of 2000 to 5000 seconds. It is within $10 \%$ of its smallest value at about 3000 seconds. Figure 6.3 shows that the confidence intervals at $495 \mathrm{~K}$ are already increasing at about 1000 seconds. In this case using the $\left|X^{T} X\right|$ criteria would be satisfactory. Table 6.2 , showing recommended heating times for the specified temperature boundary case, was constructed using information from Figures 6.1-3. Heating times were set to the time corresponding to the maximum of $\left|X^{T} X\right|$ or to where the confidence interval shape reached $10 \%$ of its minimum value.

Another method of experimental design is to maximize the minimum eigenvalue of $\boldsymbol{X}^{T} \boldsymbol{X}$. The eigenvalues for the specified temperature boundary case at $295 \mathrm{~K}$ and 495 $\mathrm{K}$ are shown in Figures 6.4 and 6.5. The minimum eigenvalue has been donated by $E 1$ in both cases. The other two are shown as E2 and E3. They are functions of the sensitivities of the parameters, as well as the way $\boldsymbol{X}^{T} \boldsymbol{X}$ is constructed. E1 reaches a minimum at times which roughly correspond to the maximum of $\left|X^{T} X\right|$. Also note that

Table 6.2 Recommended Heating Times for the Specified Temperature Boundary Case

\begin{tabular}{|c||c|}
\hline Initial Temperature & Heating Time (sec) \\
\hline \hline $295 \mathrm{~K}$ & 3000 \\
\hline $345 \mathrm{~K}$ & 2000 \\
\hline $395 \mathrm{~K}$ & 2000 \\
\hline $445 \mathrm{~K}$ & 1690 \\
\hline $495 \mathrm{~K}$ & 1500 \\
\hline
\end{tabular}




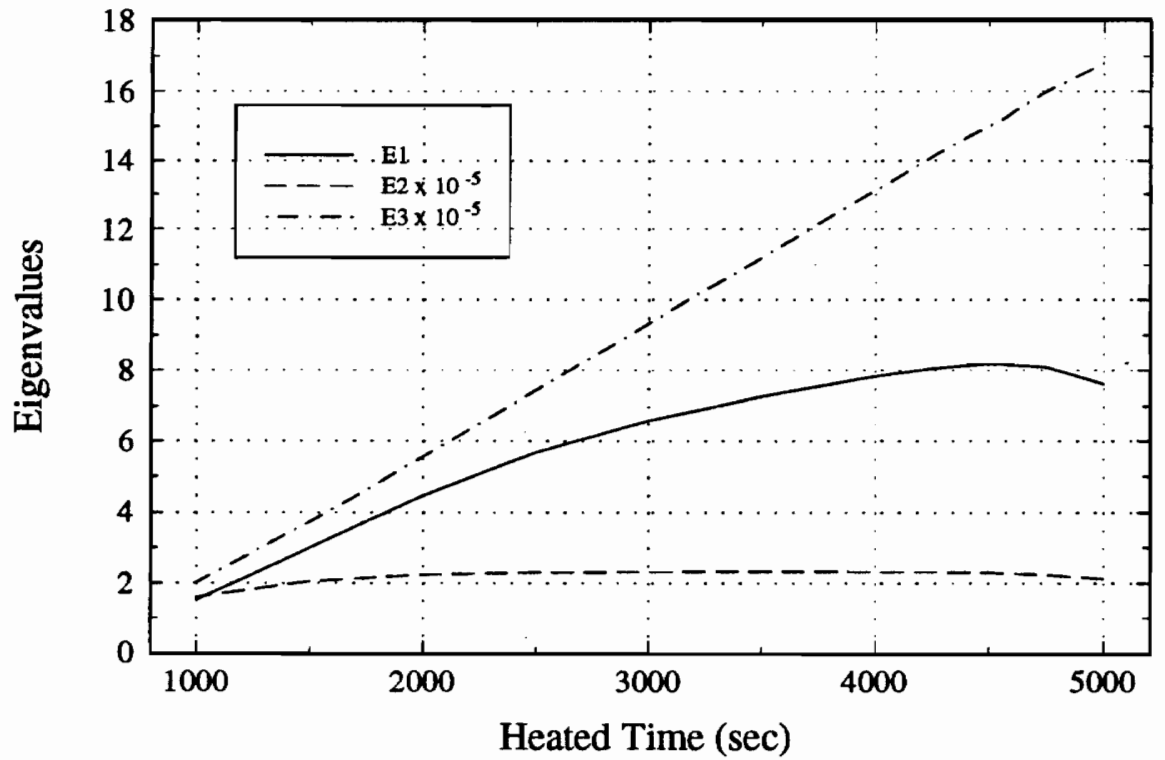

Figure 6.4 Determinant Eigenvalues at $295 \mathrm{~K}$ for the Specified Temperature Boundary Case, Used to Determine Heating Time

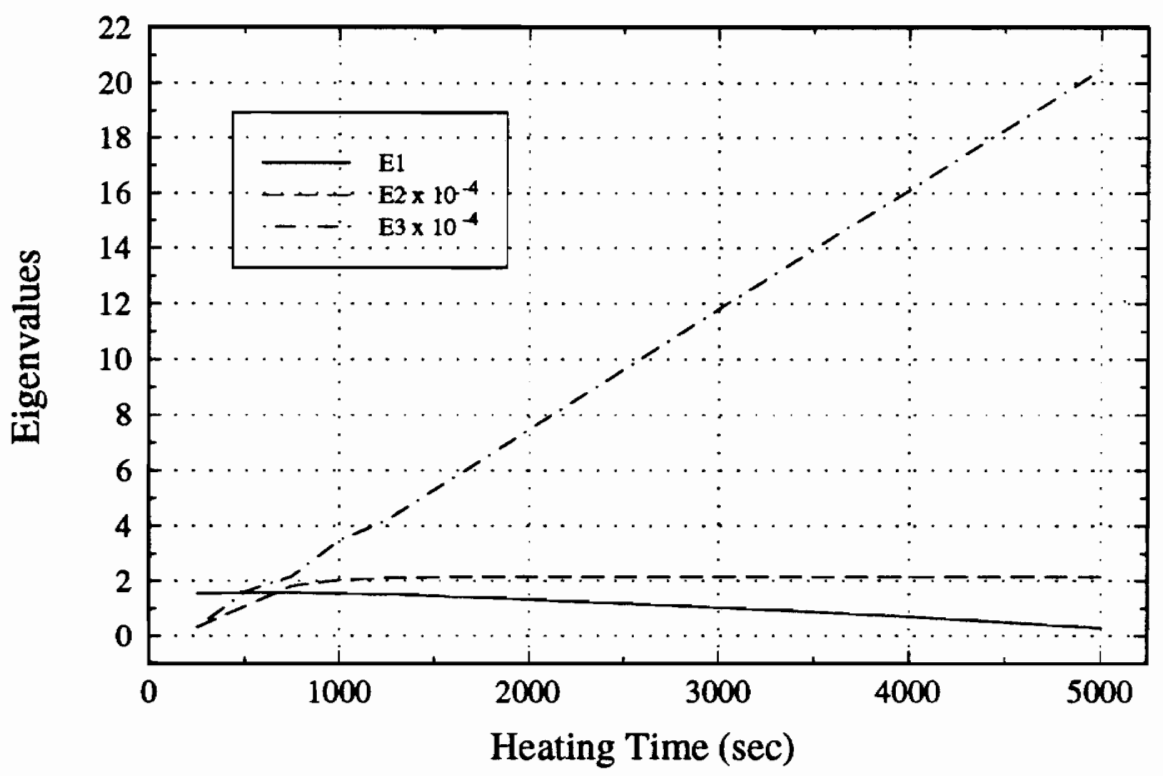

Figure 6.5 Determinant Eigenvalues at $495 \mathrm{~K}$ for the Specified Temperature Boundary Case, Used to Determine Heating Time 
$E 1$ is much smaller in magnitude than the largest eigenvalue. This indicates that the parameters are almost linearly dependent. If $E 1$ were zero, then this would be the case exactly. This explains why the correlation among the parameters is high. High correlation among parameters, as explained by Beck, usually means that the Gauss method or its modifications will not converge. That was found to be the case in this study. Plots for the other initial temperatures between $295 \mathrm{~K}$ and $495 \mathrm{~K}$ are similar and are shown in Appendix A.

The same procedure was performed for the insulated boundary case, assuming that two sensors would be used in the estimation procedure. The determinant of $\left(X^{T} X\right)$ is shown in Figure 6.6. Each curve continually increases without bound. Therefore, this criteria is useless for determining an optimum heating time.

Inspection of the scaled confidence intervals is much more useful. They are shown in Figures 6.7 and 6.8. These curves have local minima occurring near 400 seconds. They all may reach a lower value after a longer period of time, but the first minima is the one which should be used as the optimum heating time. If heating is allowed to continue to the point where the curves decrease the second time, then the sample will have reached a temperature well above its starting point. Remember that experiments were conducted at initial temperature increments of $50 \mathrm{~K}$. Allowing the sample to heat for long periods would reduce the ability to examine any temperature dependence that the parameters might have. Recommended heating times for the insulated boundary case are shown in Table 6.3. 


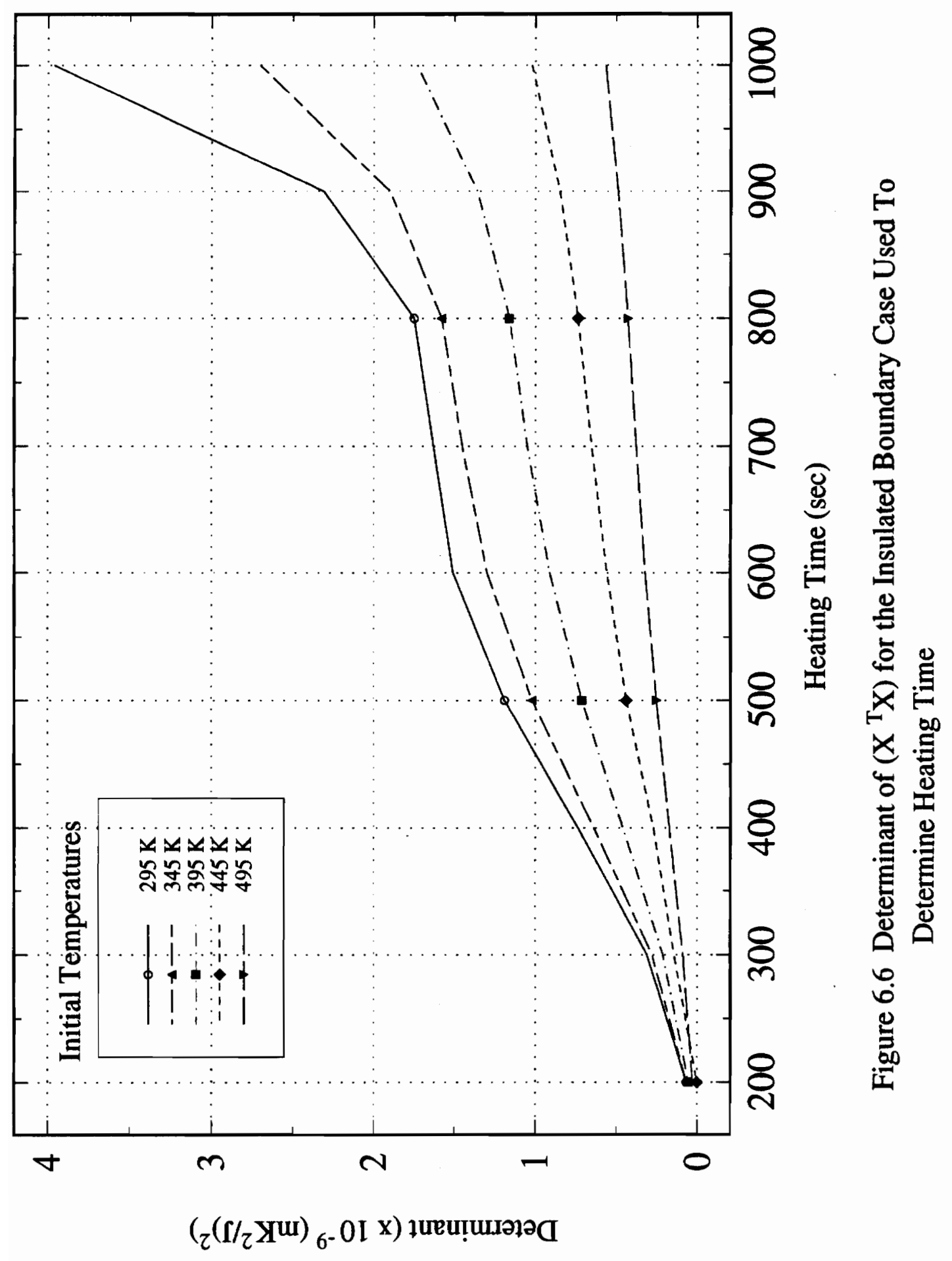




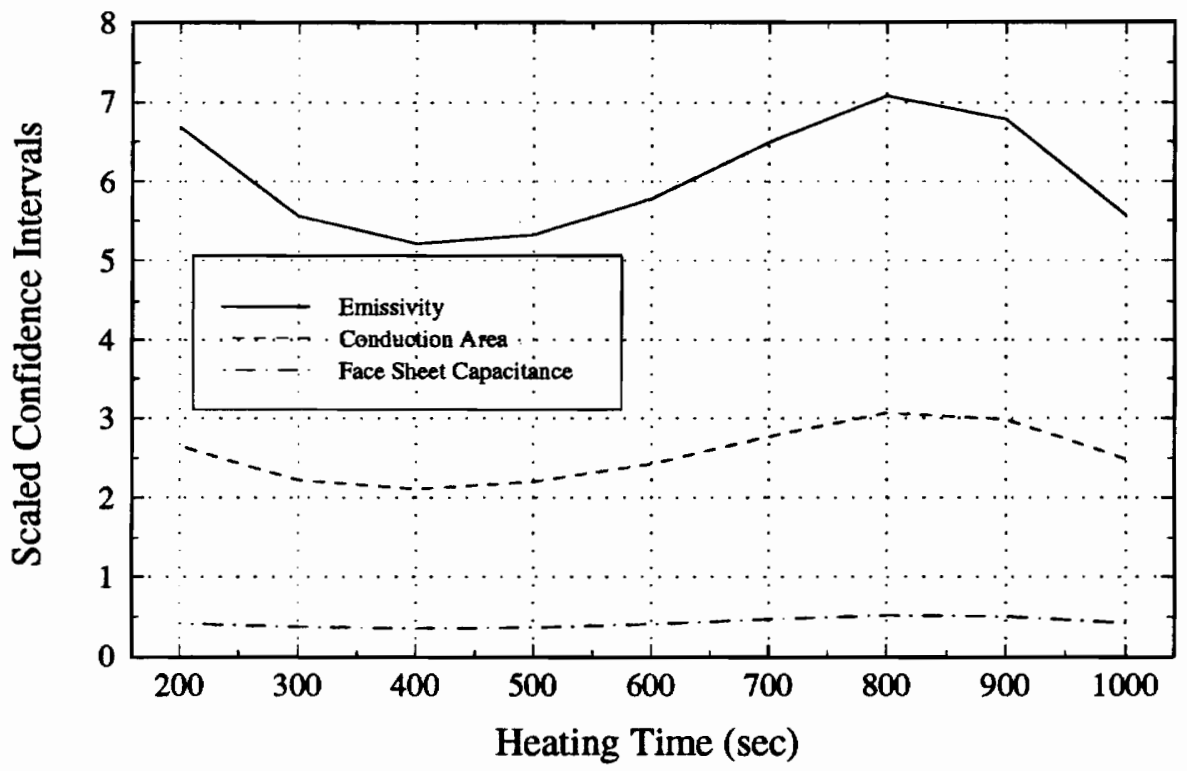

Figure 6.7 Scaled Confidence Intervals at $295 \mathrm{~K}$ for the Insulated Boundary Case, Used to Determine Heating Time

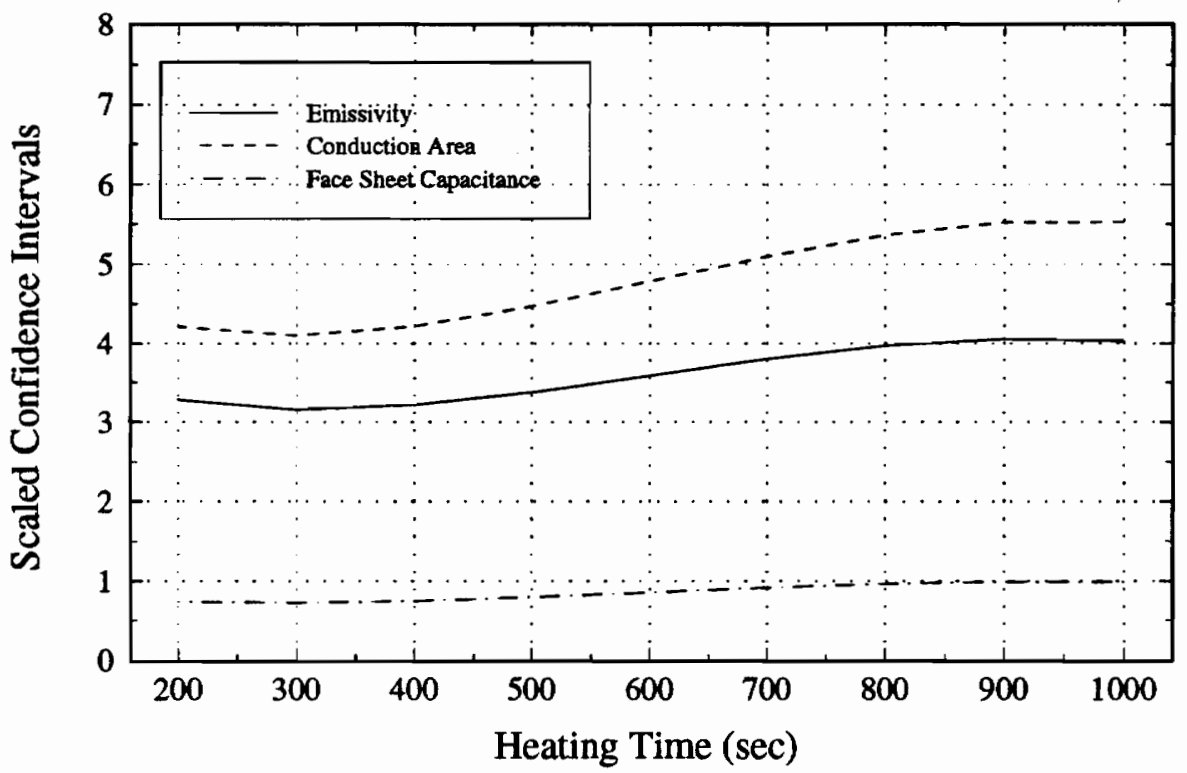

Figure 6.8 Scaled Confidence Intervals at $495 \mathrm{~K}$ for the Insulated Boundary Case, Used to Determine Heating Time 
Table 6.3 Recommended Heating Times for the Insulated Boundary Case

\begin{tabular}{||c||c||}
\hline \hline Initial Temperature & Heating Time (sec) \\
\hline \hline $295 \mathrm{~K}$ & 400 \\
\hline $345 \mathrm{~K}$ & 400 \\
\hline $395 \mathrm{~K}$ & 400 \\
\hline $445 \mathrm{~K}$ & 300 \\
\hline $495 \mathrm{~K}$ & 300 \\
\hline
\end{tabular}

The eigenvalues for the insulated boundary case reveal much the same information as the confidence interval shape plots, including the near linear dependence of the parameters. They are shown in Figures 6.9 and 6.10. Note that E1 exhibits a sinusoidal shape. This is also seen in the scaled confidence intervals. It shows up in the $\left|\boldsymbol{X}^{T} \boldsymbol{X}\right|$ graph as a change in concavity. The determinant values are not expected to go through a minimum as they are products of all three eigenvalues, and two of them are strictly increasing. Selected other graphs corresponding to this boundary condition are again shown in Appendix A.

\subsubsection{Total Experiment Time}

The next step in the design process was to determine how long the unheated portion of each experiment should be. A procedure similar to the one implemented to the find the optimum heating time was used to find the optimum total length of the experiments. However, for the $\left|\boldsymbol{X}^{T} \boldsymbol{X}\right|$ criteria, the determinant was no longer time averaged. Instead, optimum total times for this criteria were chosen to be the times where the determinants reached $95 \%$ of their maximum values. These determinants for 


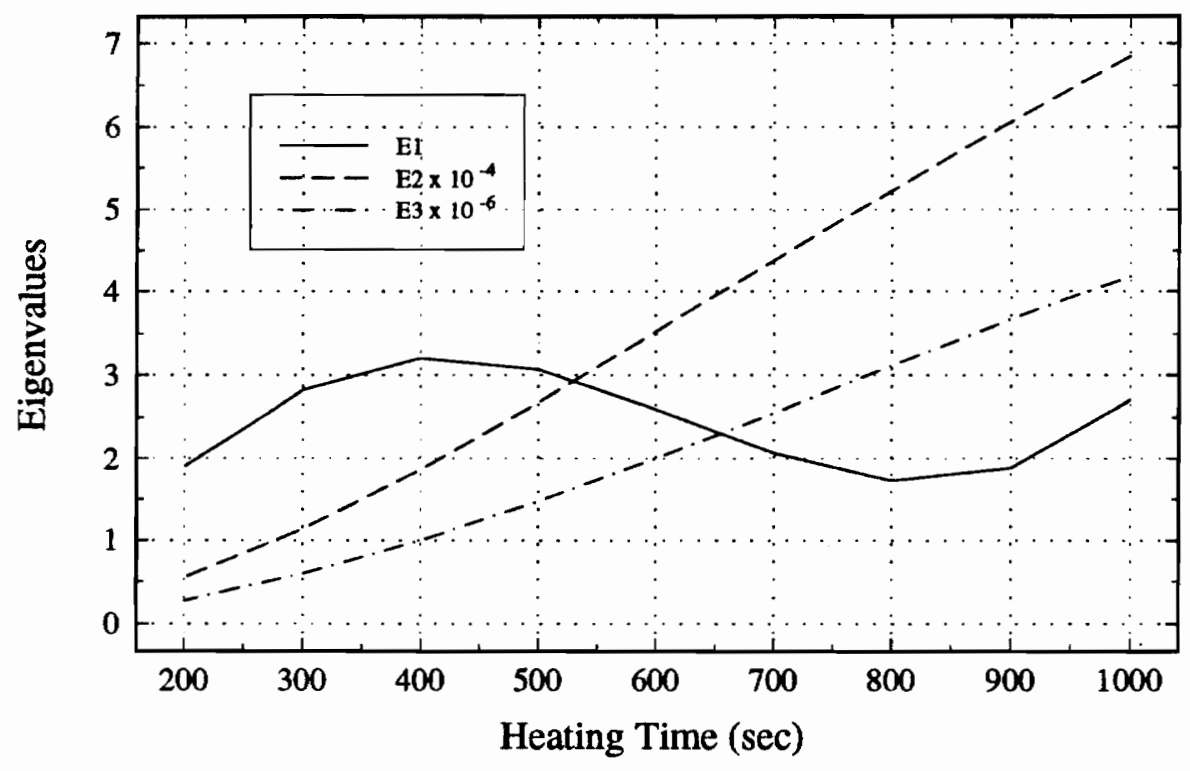

Figure 6.9 Determinant Eigenvalues at $295 \mathrm{~K}$ for the Insulated Boundary Case, Used to Determine Heating Time

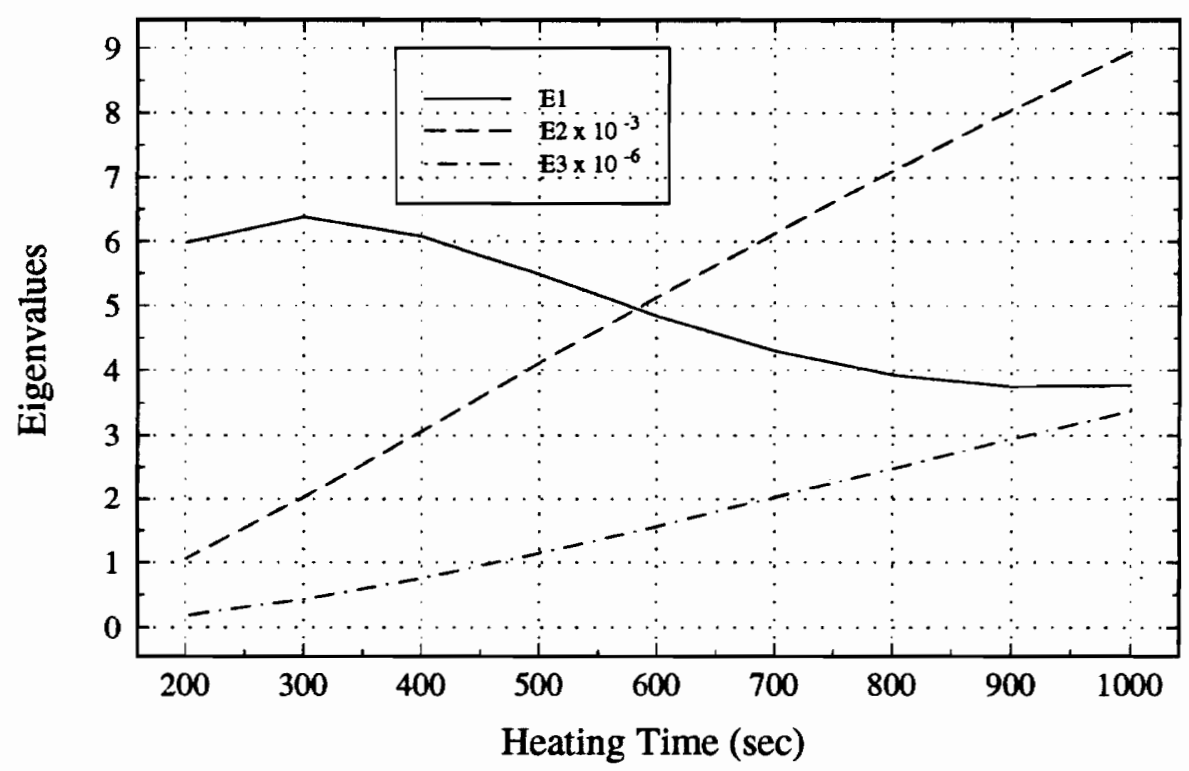

Figure 6.10 Determinant Eigenvalues at $495 \mathrm{~K}$ for the Insulated Boundary Case, Used to Determine Heating Time 
Table 6.4 Recommended Total Experiment Times for the Specified Temperature Boundary Case

\begin{tabular}{||c||c|}
\hline Initial Temperature & Total Experiment Time (sec) \\
\hline \hline $295 \mathrm{~K}$ & 4600 \\
\hline $345 \mathrm{~K}$ & 3300 \\
\hline $395 \mathrm{~K}$ & 3100 \\
\hline $445 \mathrm{~K}$ & 2600 \\
\hline $495 \mathrm{~K}$ & 2500 \\
\hline
\end{tabular}

the specified temperature boundary case are shown in Figure 6.11. Again the lower initial temperatures have larger determinants. Confidence interval shapes for $295 \mathrm{~K}$ and $495 \mathrm{~K}$ are shown in Figures 6.12 and 6.13. These curves behave similarly to the determinants. The recommended total experiment times are shown in Table 6.4. Figure 6.14 shows the determinants for the insulated boundary case. These continually increase as before. They do not appear asymptotic at times up to 2000 seconds. Continued data collection after this much time would be highly suspect, due to the fact that the insulated boundary could no longer be modelled as truly insulated. The confidence interval shapes in Figures 6.15 and 6.16 decrease slowly after about 800 seconds. This suggests that any improvements in the estimates which could be made by collecting data in the 1500-2000 second range would be minimal. The optimum total experiment times for this case were chosen to be the times when the largest confidence interval reached about $30 \%$ of its value at 2000 seconds. These values are listed in Table 6.5. 


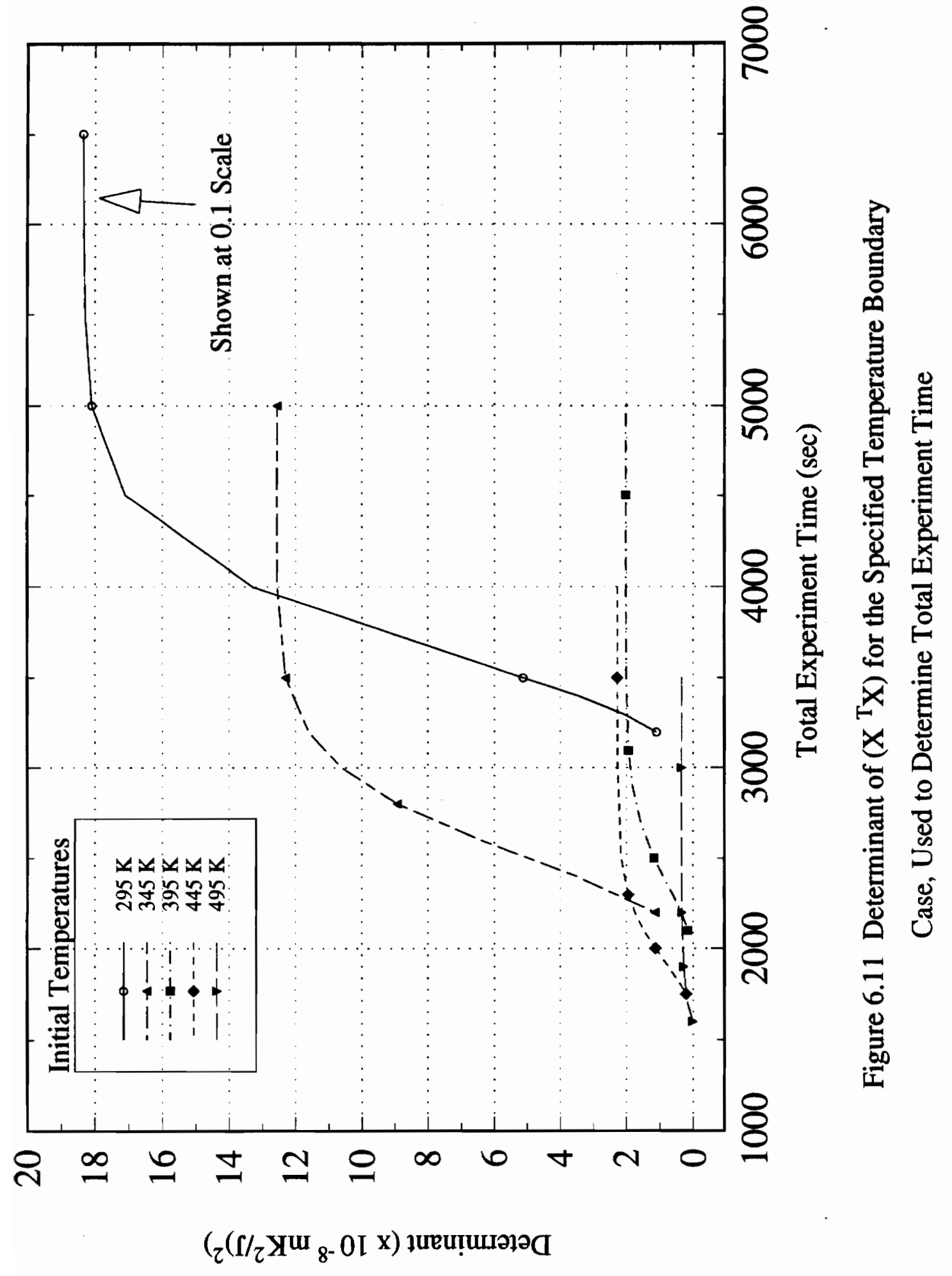




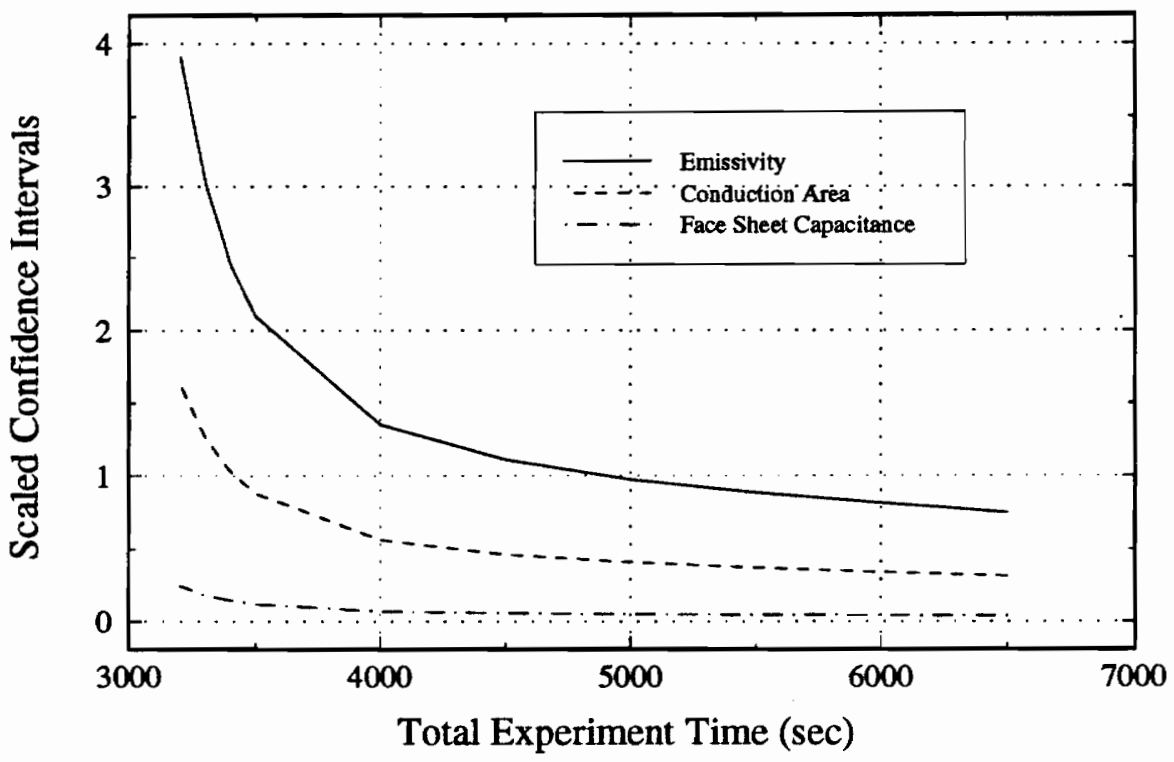

Figure 6.12 Scaled Confidence Intervals at $295 \mathrm{~K}$ for the Specified Temperature Boundary Case, Used to Determine Total Experiment Time

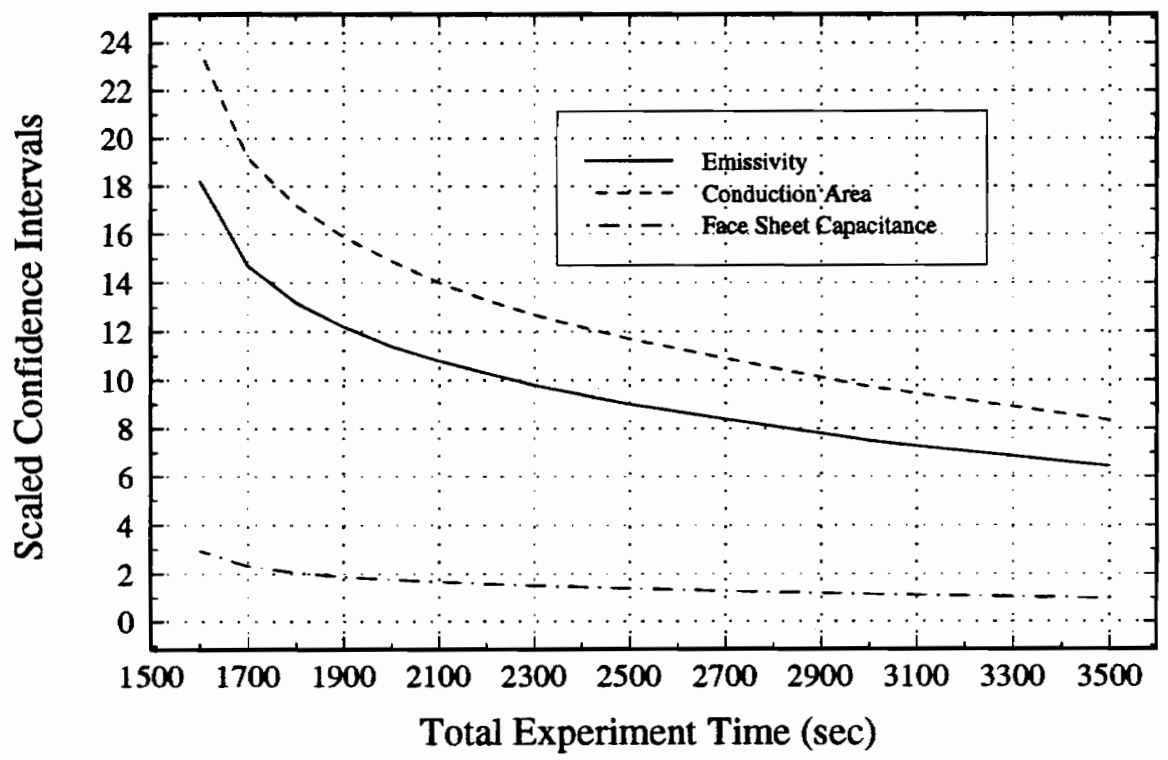

Figure 6.13 Scaled Confidence Intervals at $495 \mathrm{~K}$ for the Specified Temperature Boundary Case Used to Determine Total Experiment Time 


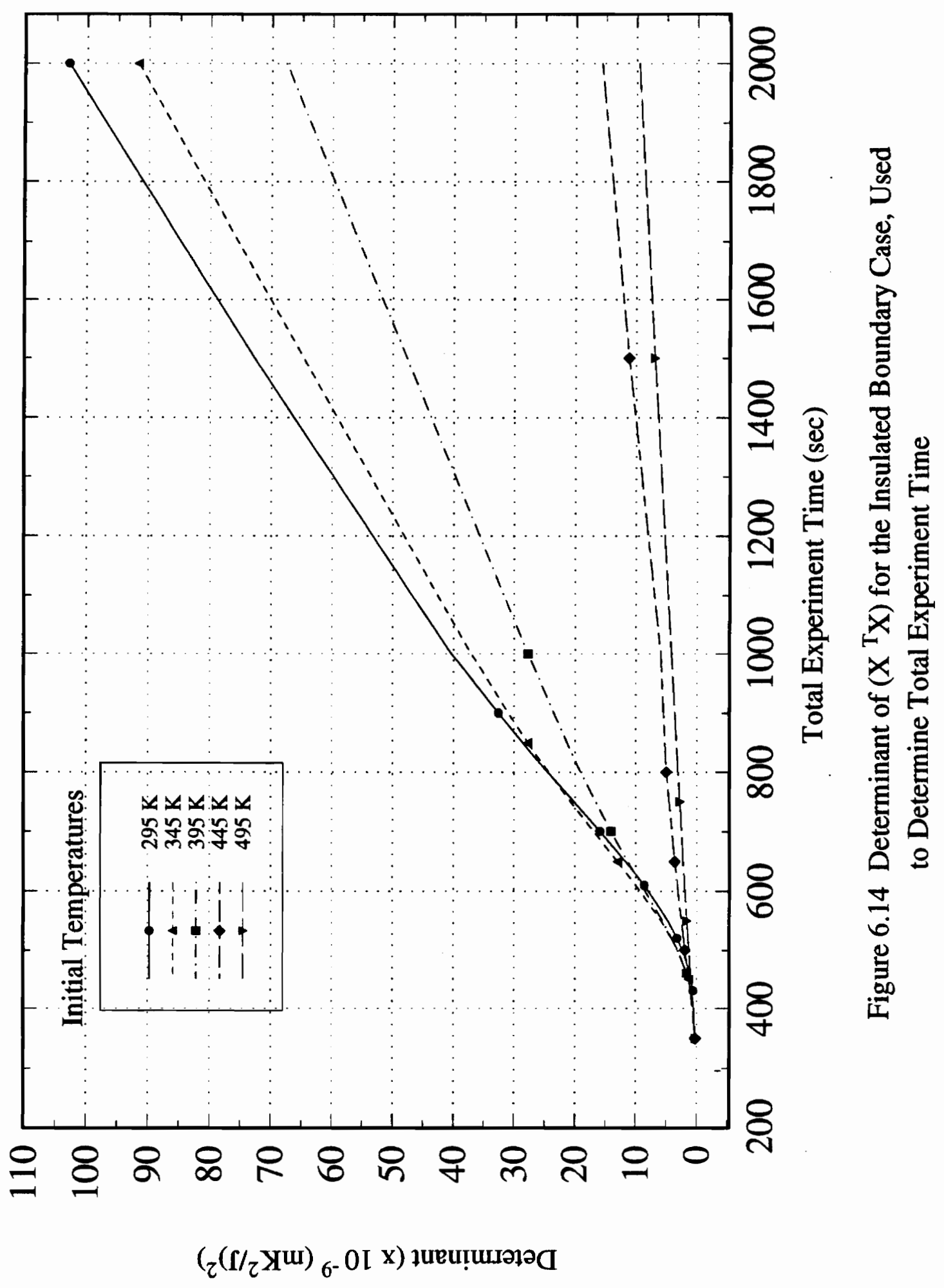




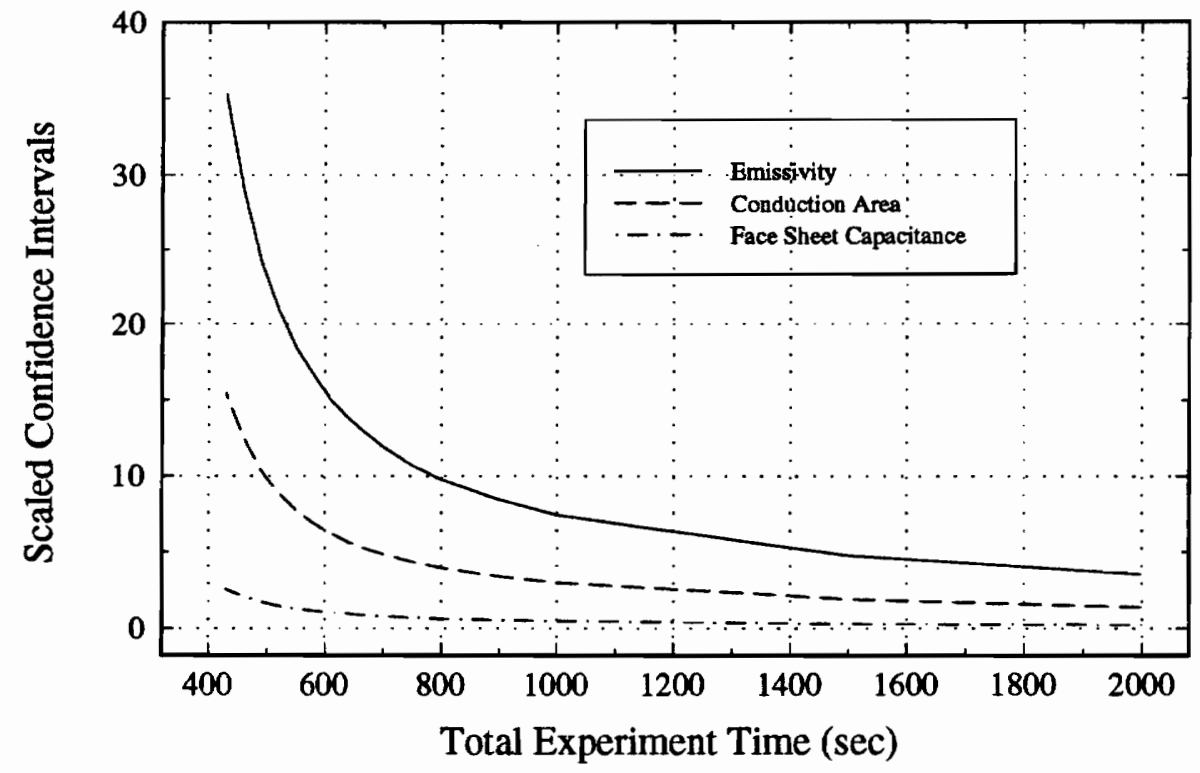

Figure 6.15 Scaled Confidence Intervals at $295 \mathrm{~K}$ Used to Determine Total Experiment Time for the Insulated Boundary Case

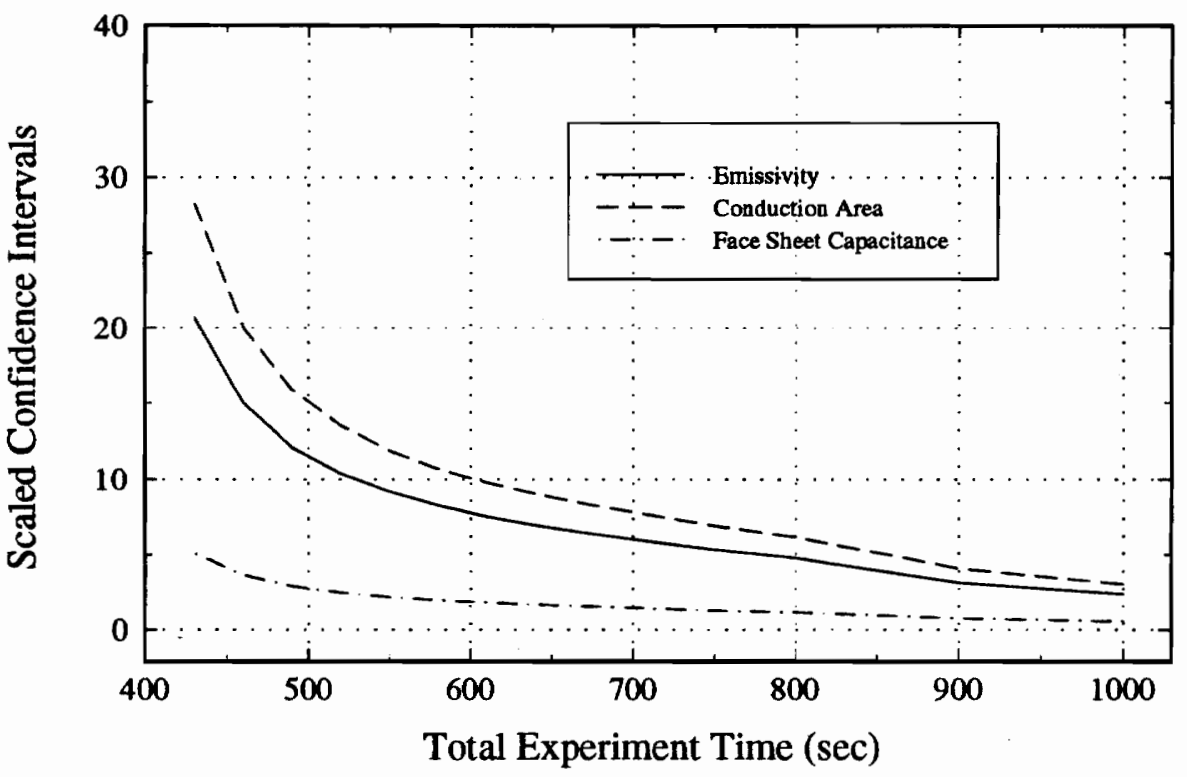

Figure 6.16 Scaled Confidence Intervals at $495 \mathrm{~K}$ Used to Determine Total Experiment Time for the Insulated Boundary Case 
Table 6.5 Recommended Total Experiment Times for the Insulated Boundary Case

\begin{tabular}{||c||c||}
\hline Initial Temperature & Total Experiment Time (sec) \\
\hline \hline $295 \mathrm{~K}$ & 1630 \\
\hline $345 \mathrm{~K}$ & 1560 \\
\hline $395 \mathrm{~K}$ & 1500 \\
\hline $445 \mathrm{~K}$ & 1460 \\
\hline $495 \mathrm{~K}$ & 1400 \\
\hline
\end{tabular}

\subsubsection{Other Factors}

The main focus of the experimental design procedure was to find the optimum heating and total experiment times for all boundary conditions. Another factor which could have been optimized was the magnitude of the applied heat flux. It was found that in all cases more information and better estimates resulted from the higher temperature gradients associated with higher heat fluxes. Therefore, the highest heat flux that the available power supply could produce was used. This corresponded to about $350 \mathrm{~W} / \mathrm{m}^{2}$.

It would also be possible to vary the shape of the heat flux. A step function was used in this study. The sudden change associated with the step produced the most sensitive information, especially for volumetric heat capacity. The number of heat pulses per experiment could also be varied. Instead of doing this, however, it would probably be better to run a number of single-pulse experiments.

Optimization routines could be performed on sensor location or variable 
boundary conditions. However, it would be almost physically impossible to locate a sensor somewhere other than a surface. In addition, sensitivities are usually highest at surfaces. A radiative or conductive boundary could have been analyzed, but again this is not practical. A radiative boundary is difficult to achieve, and convection heat transfer has so many uncertainties that estimation of heat transfer coefficients is a topic which should be performed by itself. The two possible boundary conditions were analyzed as separate cases.

\subsection{Sensitivity Coefficients}

The sensitivity coefficients of an experiment give insight into the ability to perform accurate parameter estimation. In this study they represent the derivative of temperature with respect to the unknown thermal properties. The best parameter estimates will result when the sensitivity coefficients are large in magnitude and are linearly independent. Sensitivity coefficients at the heated surface of the specified temperature boundary case at $295 \mathrm{~K}$ and $495 \mathrm{~K}$ are shown in Figures 6.17 and 6.18. They have been made dimensionless by dividing by the maximum temperature rise, $26 \mathrm{~K}$. They both correspond to a heated time of 1500 seconds and a total experiment time of 2500 seconds for purposes of comparison.

Notice that the magnitudes of the sensitivity coefficients generally become smaller with increasing temperature. This indicates that better estimates may be obtained at lower temperatures. This is despite the fact that the proportion of heat transferred by radiation will be greater at the higher temperatures. The magnitude of the emissivity sensitivity coefficient is the exception. It remains nearly specified, while the magnitudes 


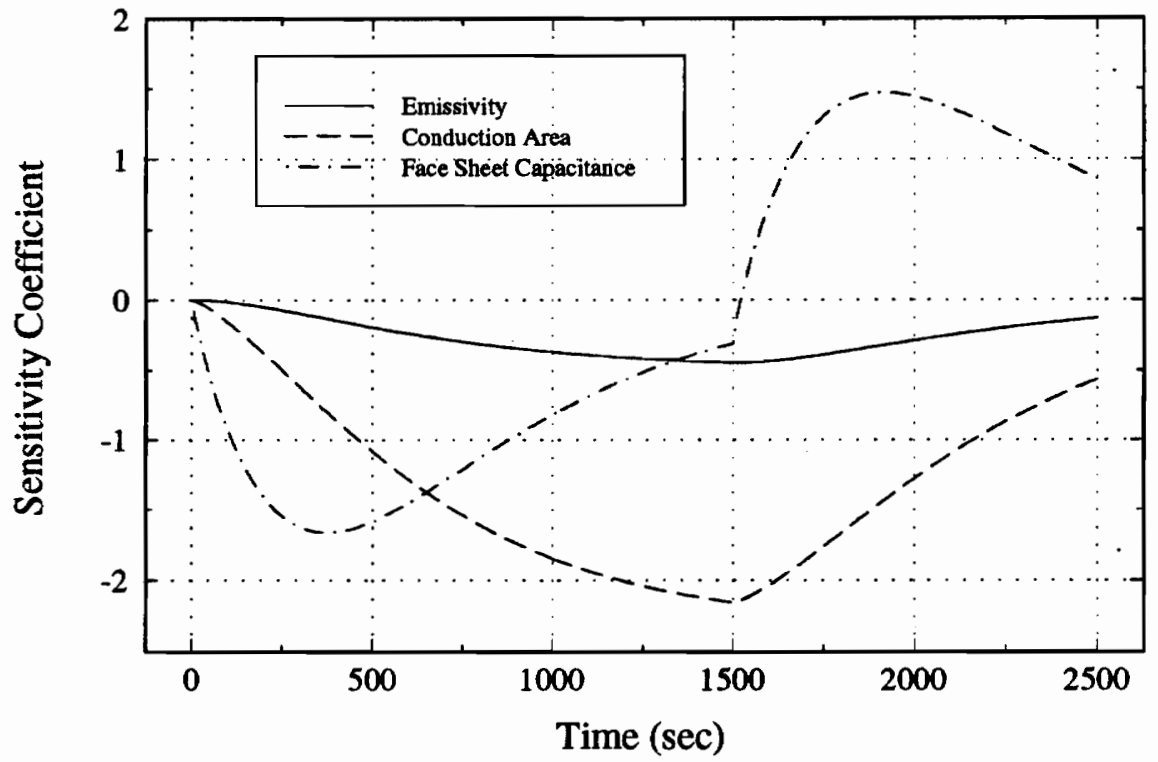

Figure 6.17 Dimensionless Sensitivity Coefficients for the Specified Temperature Boundary Case at $295 \mathrm{~K}$ at the Heated Surface

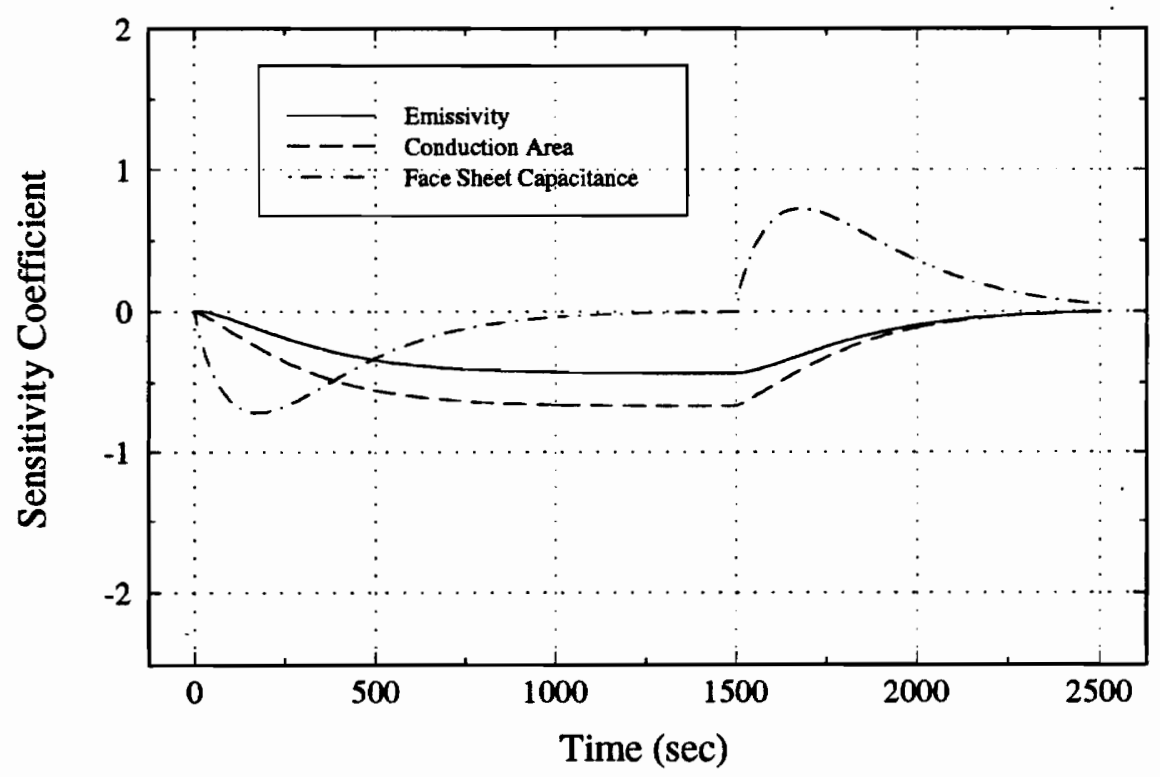

Figure 6.18 Dimensionless Sensitivity Coefficients for the Specified Temperature Boundary Case at $495 \mathrm{~K}$ at the Heated Surface 
of the other two decrease. This may be due to the fact that the thermal conductivity of the structure increases by over $40 \%$ from $295 \mathrm{~K}$ to $495 \mathrm{~K}$. An increase in the thermal conductivity would allow heat to be transferred more easily, thus producing a smaller temperature gradient through the structure. This would reduce the sensitivity of conduction area and face sheet capacitance.

The shapes of the curves indicate whether or not linear dependence, and therefore correlation, exist. The shape of the face sheet capacitance curve is greatly different from the other two. It approaches zero near the steady-state heating and cooling of the sample, and shows a rapid increase near the beginning of the experiment and just after heating is stopped. The purpose of collecting data during both heating and cooling of the sample was to take advantage of information gained by this procedure. The emissivity and face sheet capacitance curves, however, look very much alike. This indicates that there is correlation between the two. Better estimates might result if these curves were more dissimilar.

The sensitivity coefficients for the insulated boundary case are shown in Figures 6.19 and 6.20 for a heating time of 450 seconds and a total time of 800 seconds. There are six sensitivity coefficients, one for each parameter at each surface. All sensitivity coefficients corresponding to emissivity and conduction area are generally of the same shape and low in magnitude. This is due to the smaller temperature gradients that are associated with this boundary condition. The coefficients corresponding to face sheet capacitance are larger, but their shape resembles the other two. These facts indicate that estimates from this boundary condition may be difficult to obtain and may not be as accurate as those obtained by the specified temperature boundary model. 


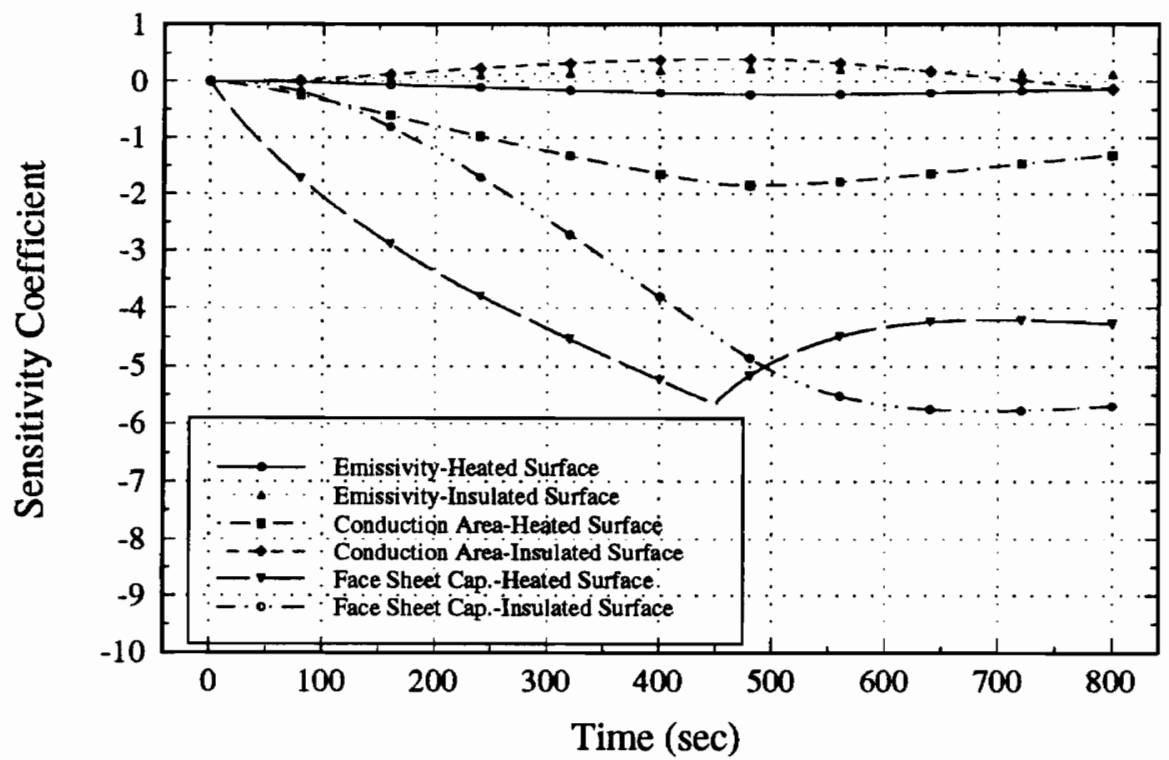

Figure 6.19 Dimensionless Sensitivity Coefficients for the Insulated Boundary Case at $295 \mathrm{~K}$ at Both Surfaces

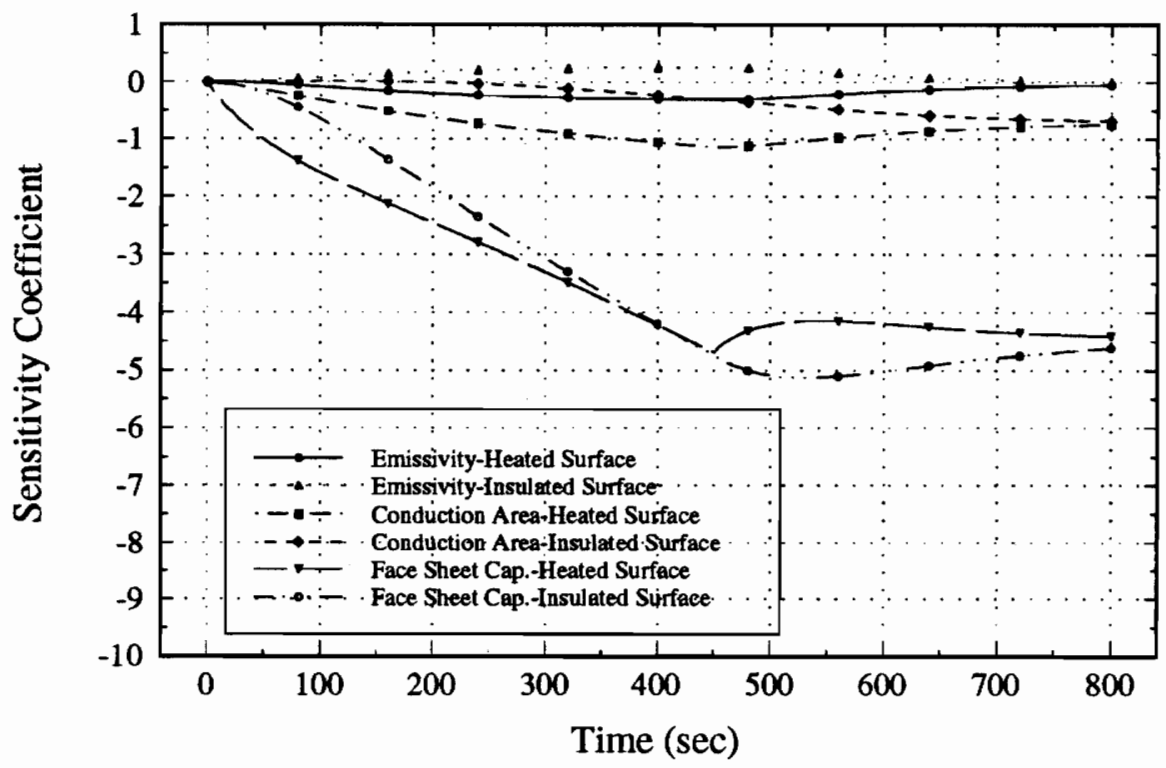

Figure 6.20 Dimensionless Sensitivity Coefficients for the Insulated Boundary Case at $495 \mathrm{~K}$ at Both Surfaces 


\subsection{Estimation Results}

Four estimation models were examined. These include the specified temperature boundary case (Case 1), the insulated boundary case with one (Case 2) or two (Case 3) sensors, and the case of estimation using both specified temperature boundary data and insulated boundary (Case 4) data. These estimation models are reviewed in Figure 6.21. Data collected for use in the estimation procedure was based closely on the experimental design parameters for the $495 \mathrm{~K}$ cases. Time constraints prohibited the full development of the experimental design procedure before data collection took place. This section presents the estimates along with $95 \%$ confidence intervals for the mean of a set of estimates. This confidence interval is for the mean of a set corresponding to temperature and model only and should not be confused with the confidence intervals which result directly from the estimation procedure performed on each experiment.

\subsubsection{Specified Temperature Boundary Results (Case 1)}

The estimates obtained using the specified temperature boundary are shown in Figures 6.22-24. These graphs are all error bars, each indicating the value of the estimated property for each experiment and its $95 \%$ confidence interval resulting from estimation. Emissivity estimates are all within or near the $0.7-1.0$ range, but the confidence intervals are large. Recall that constraints were placed on the estimation routine to limit $\varepsilon$ to values between 0.4 and 1.0. Some confidence intervals exceed these limits. Conduction area estimates and confidence regions are all located near the $0.3 \times$ $10^{-4}$ to $0.5 \times 10^{-4}$ range. Small confidence regions are also noticed for the volumetric heat 

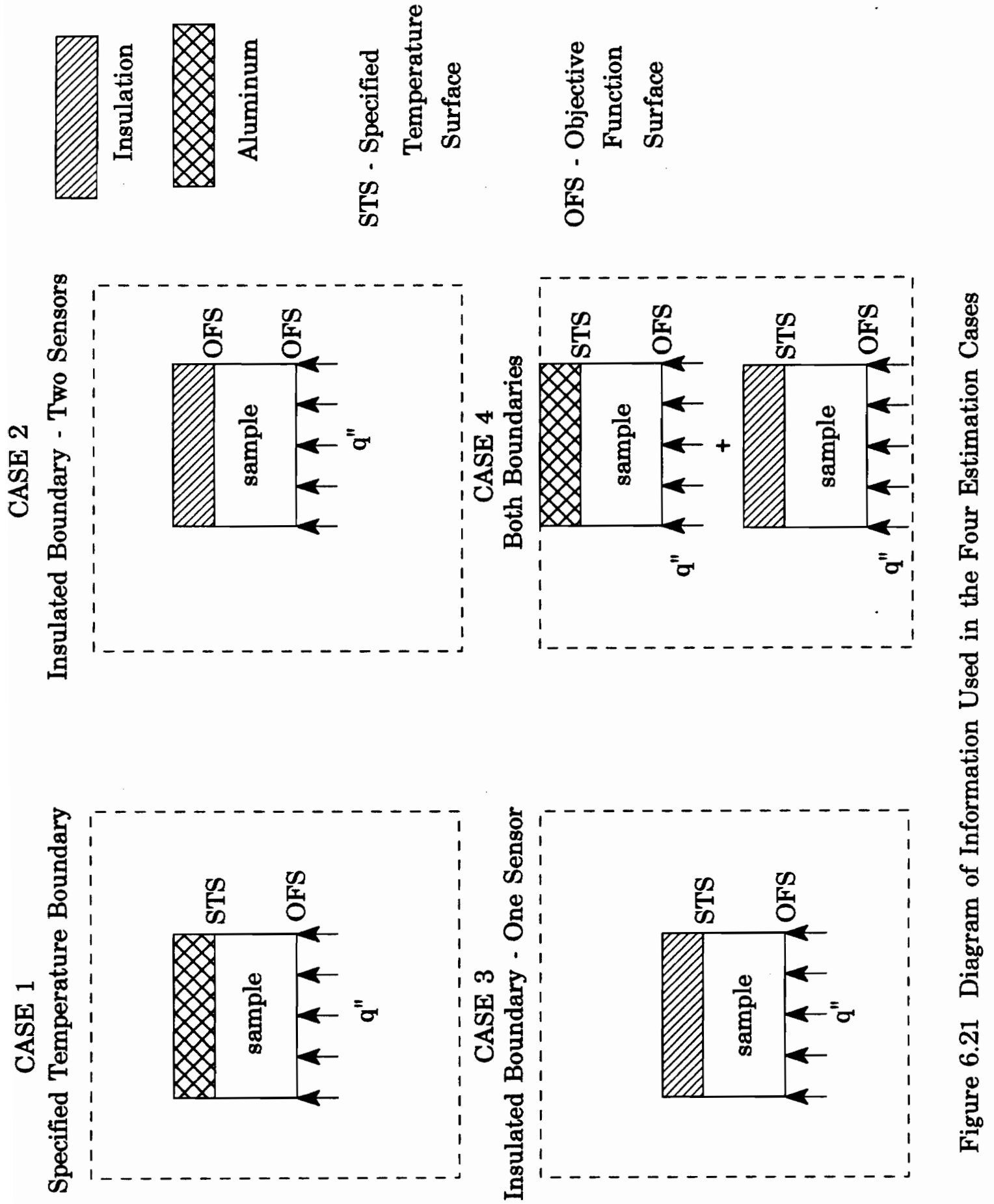


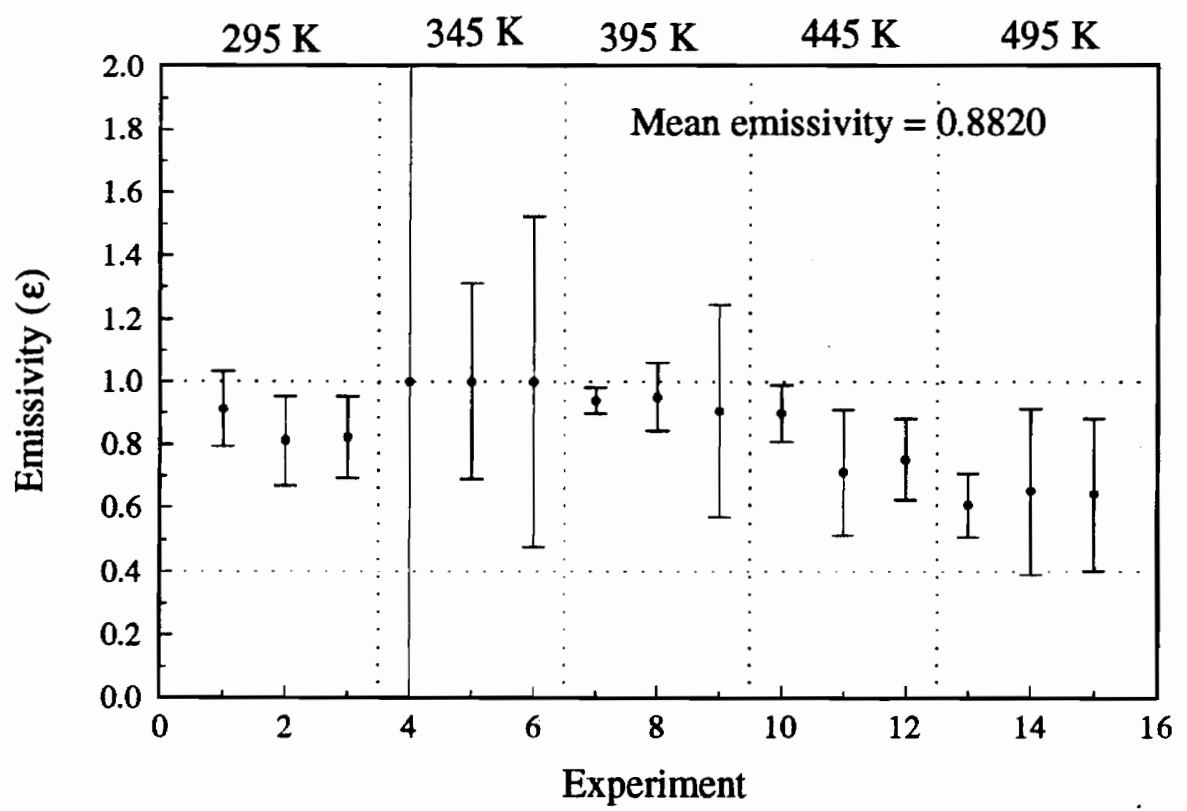

Figure 6.22 Emissivity Estimates for the Specified Temperature Boundary Condition

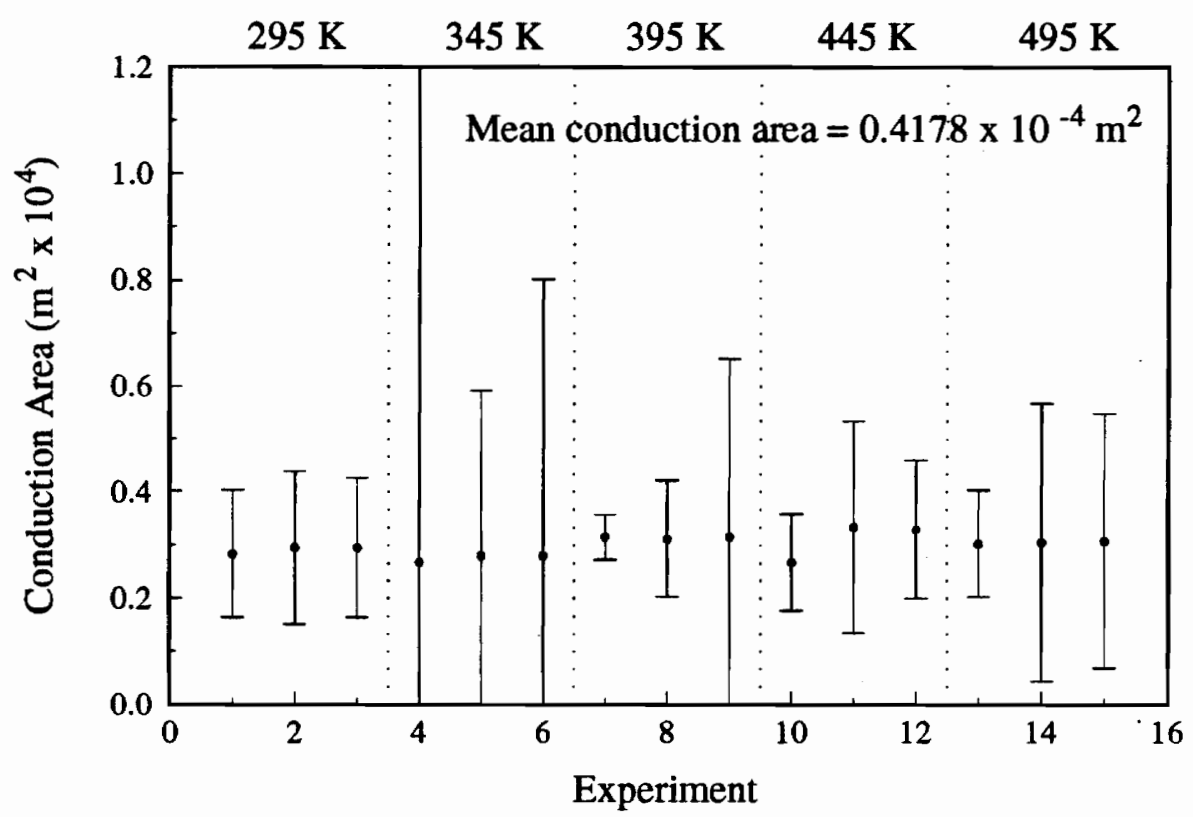

Figure 6.23 Conduction Area Estimates for the Specified Temperature Boundary 


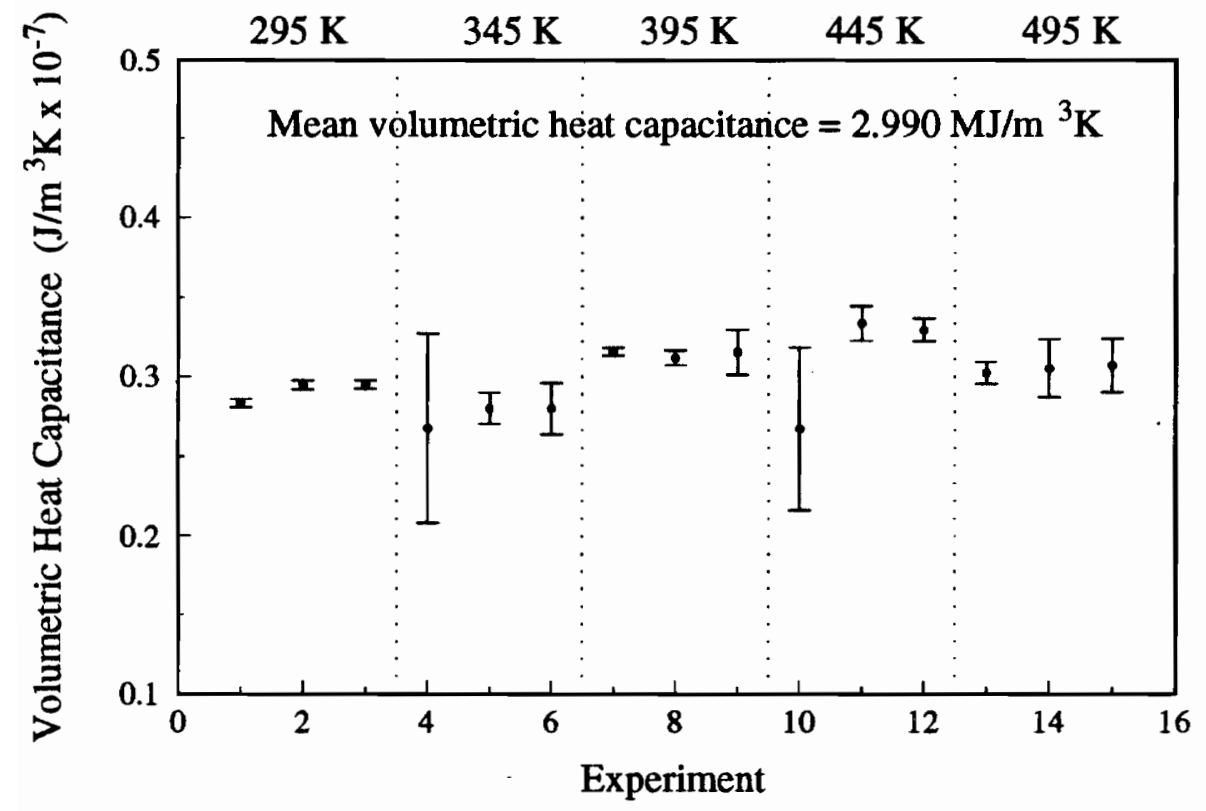

Figure 6.24 Volumetric Heat Capacity Estimates for the Specified Temperature Boundary 
Table 6.6 Mean estimate values for all Initial Temperatures for the Specified Temperature Boundary Case

\begin{tabular}{|l||l||}
\hline Parameter & Estimate Value \\
\hline \hline Emissivity & $0.8820 \pm .01017$ \\
\hline Conduction Area & $0.4178 \pm 0.0417 \times 10^{-4} \mathrm{~m}^{2}$ \\
\hline Volumetric Heat Capacity & $0.2990 \pm 0.0114 \times 10^{7} \mathrm{~J} / \mathrm{m}^{3} \mathrm{~K}$ \\
\hline
\end{tabular}

capacity estimates. Experiments four and ten seem to have a greater amount of error, as seen by their large confidence intervals. Recall that the area was determined to be about $3.5 \times 10^{-5} \mathrm{~m}^{2}$ from actual measurements. The estimated value is within $15 \%$ of this, indicating that the result is reasonable. Descriptive statistics are shown for this case in Table 6.6.

\subsubsection{Estimation Results for the Insulated Boundary With Two Sensors (Case 2)}

For Case 2, information from both sensors was used in the objective function. The insulation at the outer boundary was modelled, with the outer boundary of the insulation assumed to be perfectly insulated. Results from this estimation procedure are shown graphically in Figures 6.25-27. Emissivity estimates have small confidence intervals but are clustered near the 0.4 constraint, except for the $445 \mathrm{~K}$ case which has higher values but extremely wide confidence intervals. Conduction area and volumetric heat capacity estimates generally have small confidence intervals which do not overlap. The volumetric heat capacity estimates appear to be increasing as a function of temperature, while conduction area estimates are random. Descriptive statistics are shown in Table 6.7 . 


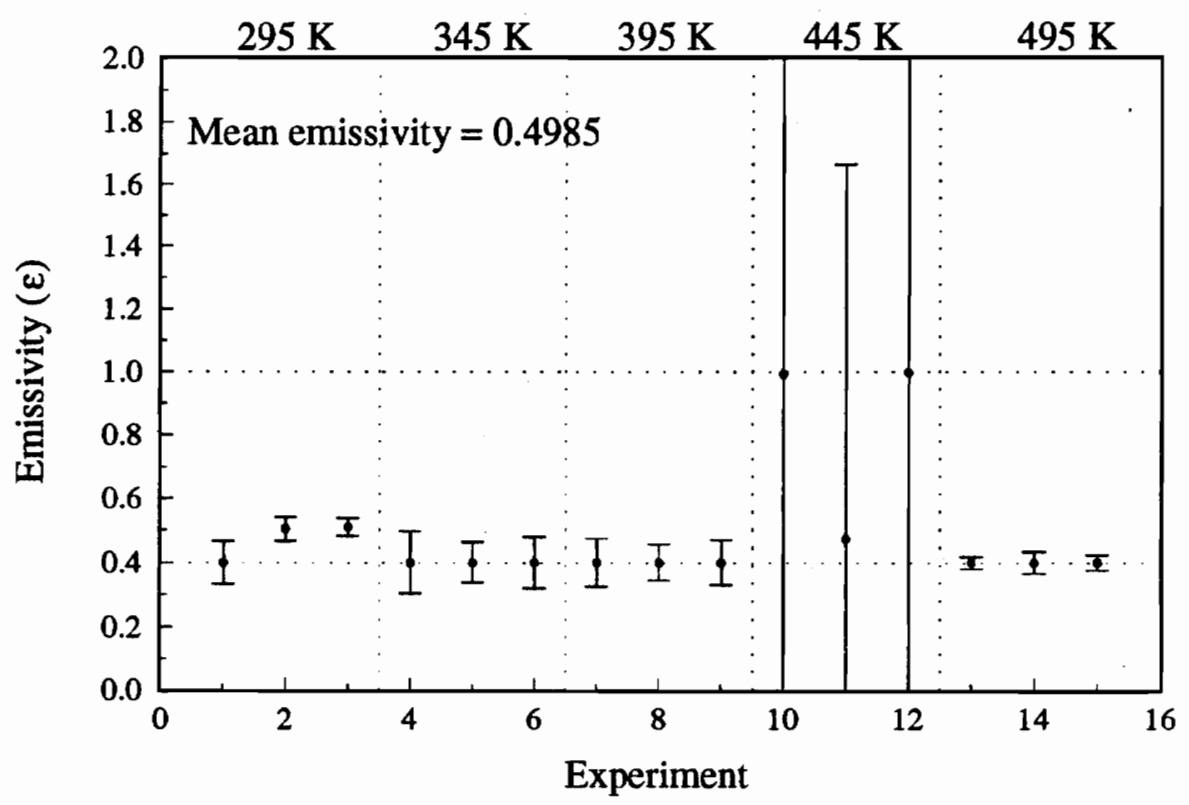

Figure 6.25 Emissivity Estimates for the Insulated Boundary Condition With Two Sensors

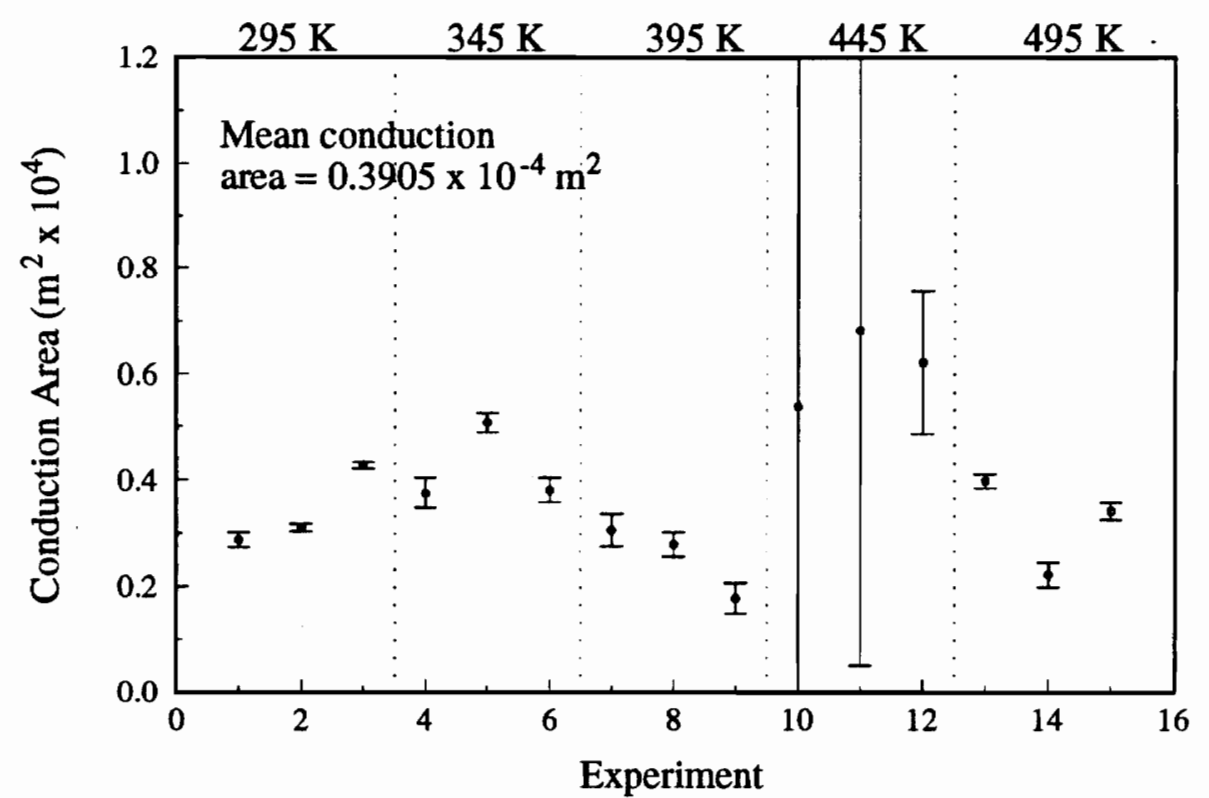

Figure 6.26 Conduction Area Estimates for the Insulated Boundary Condition With Two Sensors 


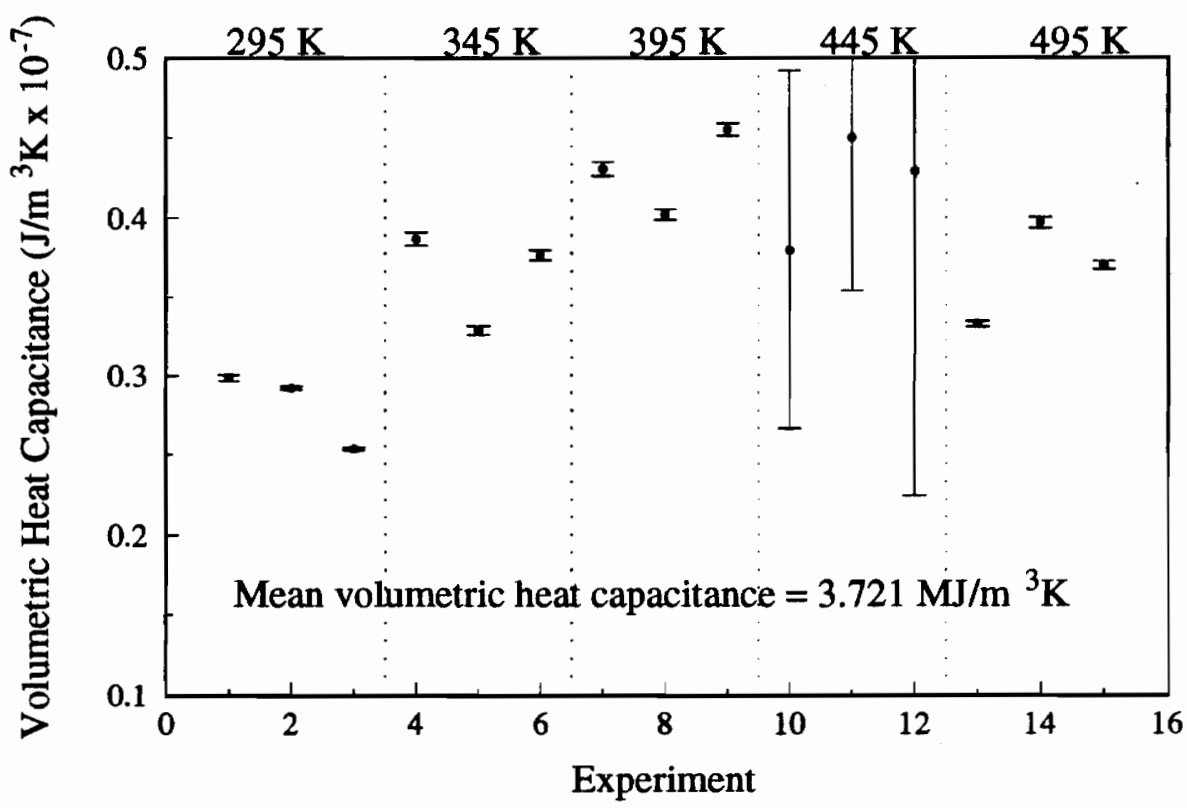

Figure 6.27 Volumetric Heat Capacity Estimates for the Insulated Boundary Condition With Two Sensors 
Table 6.7 Mean Estimate Values at all Initial Temperatures for the Insulated Boundary Case Using Information From Two Sensors.

\begin{tabular}{||l||l||}
\hline Parameter & Estimate Value \\
\hline \hline Emissivity & $0.4985 \pm 0.1138$ \\
\hline Conduction Area & $0.3905 \pm 0.0794 \times 10^{-4} \mathrm{~m}^{2}$ \\
\hline Volumetric Heat Capacity & $0.3721 \pm 0.3333 \times 10^{7} \mathrm{~J} / \mathrm{m}^{3} \mathrm{~K}$ \\
\hline
\end{tabular}

\subsubsection{Estimation Results for the Insulated Boundary With One Sensor (Case 3)}

The estimation procedure was performed again on the insulated boundary data using only information from the sensor at the heated surface in the objective function. It was hoped that this would relieve problems caused by errors in the unknown temperature distribution of the insulation. The unheated boundary was treated as it was in the true specified temperature boundary case and was not included in the objective function as in Case 2.

Results are shown in Figures 6.28-30 and in Table 6.8. Emissivity estimates for this case are all near the 1.0 constraint instead of the 0.4 and have very large confidence intervals which encompass almost all of the entire allowable range. Conduction area estimates are scattered and have large confidence intervals. The volumetric heat capacity estimates are mostly within the 0.25 to 0.3 range with the smallest confidence interval of any estimates. 


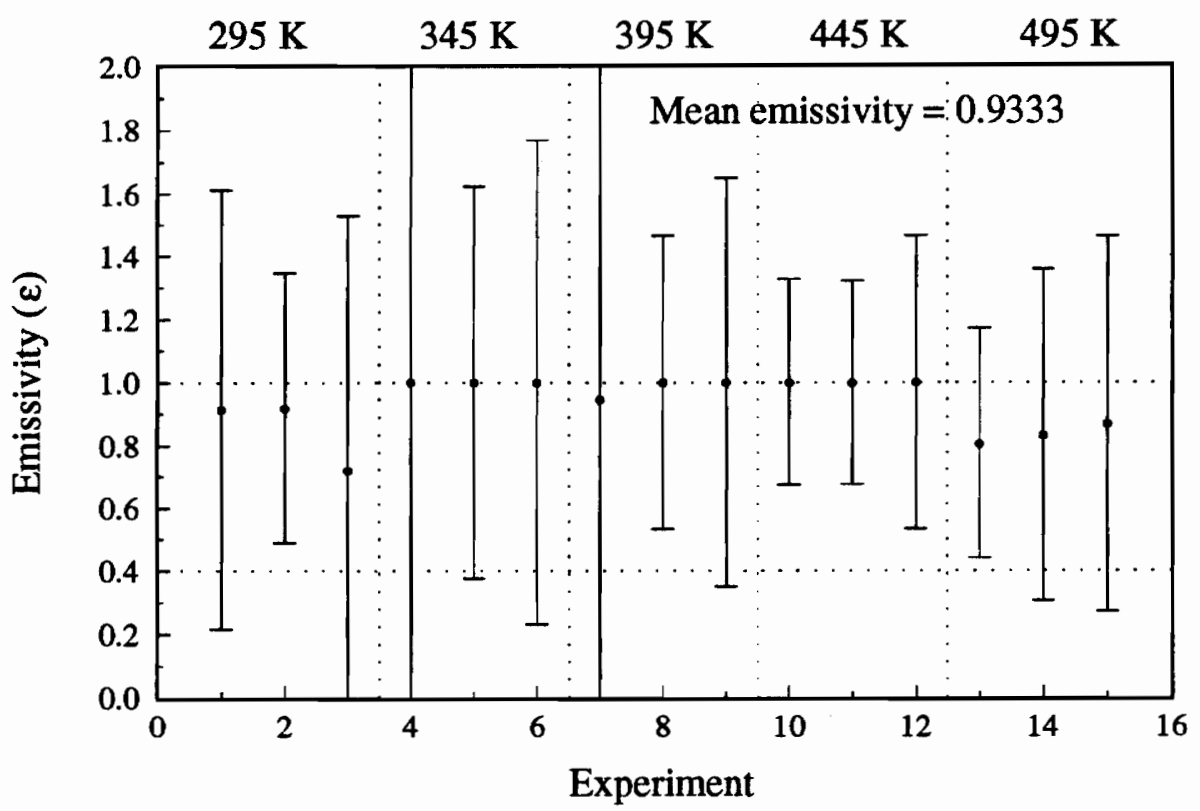

Figure 6.28 Emissivity Estimates for the Insulated Boundary Condition With One Sensor

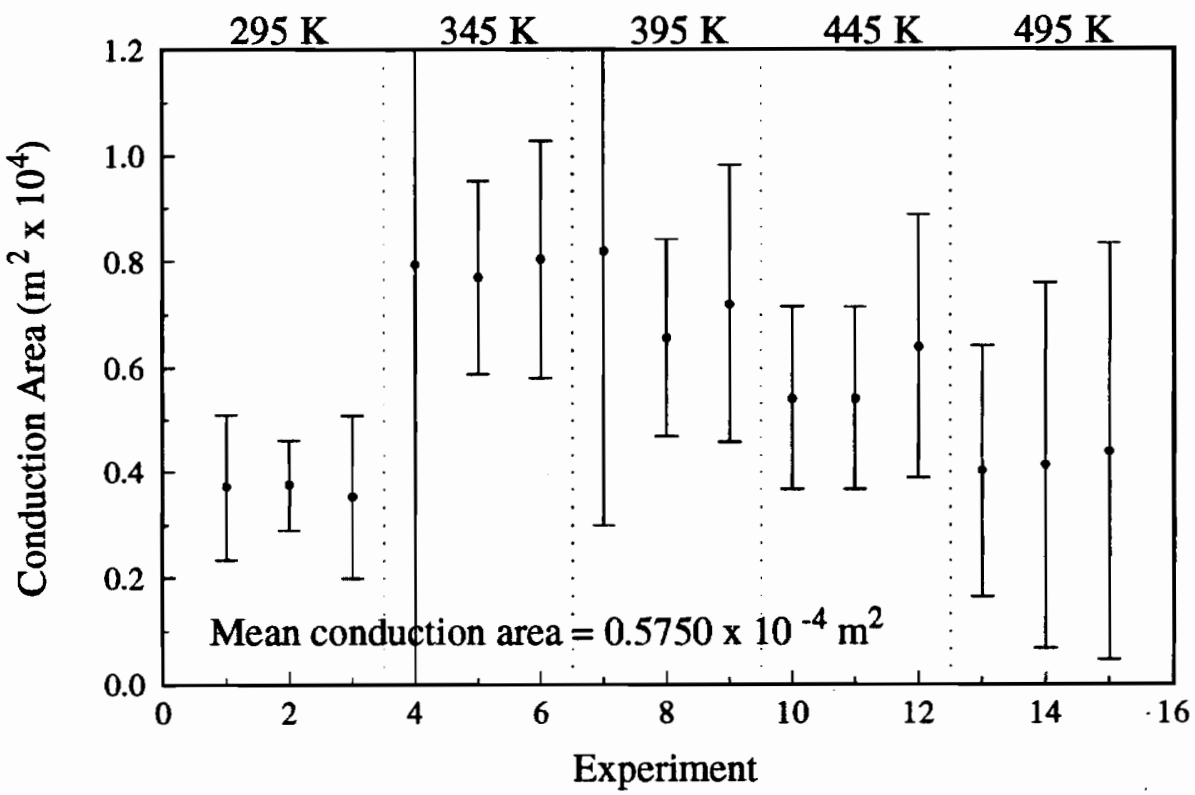

Figure 6.29 Conduction Area Estimates for the Insulated Boundary Condition With One Sensor 


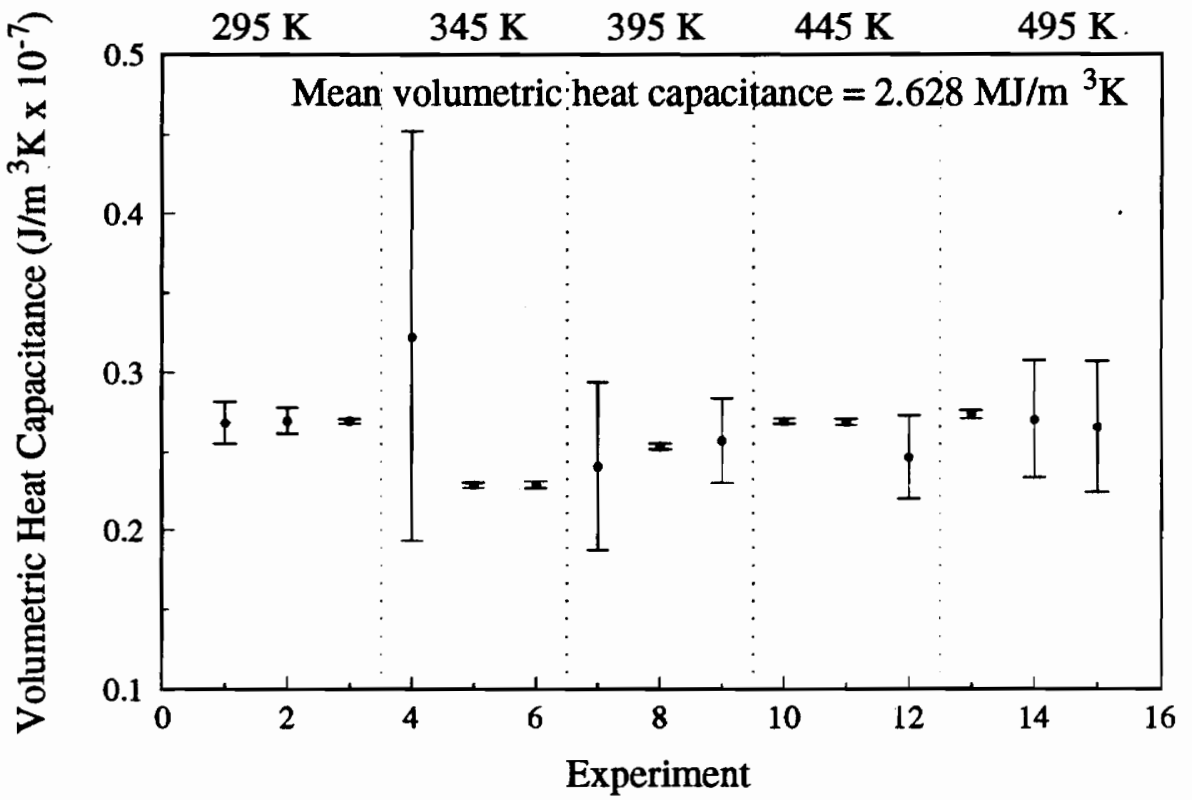

Figure 6.30 Volumetric Heat Capacity Estimates for the Insulated Boundary Condition With One Sensor 
Table 6.8 Mean Estimate Values at all Initial Temperatures for the Insulated Bound ary Case Using Information From One Sensor

\begin{tabular}{|l||l||}
\hline \hline Parameter & Estimate Value \\
\hline \hline Emissivity & $0.9333 \pm 0.0497$ \\
\hline Conduction Area & $0.5760 \pm 0.0979 \times 10^{-4} \mathrm{~m}^{2}$ \\
\hline Volumetric Heat Capacity & $0.2628 \pm 0.0129 \times 10^{7} \mathrm{~J} / \mathrm{m}^{3} \mathrm{~K}$ \\
\hline
\end{tabular}

\subsubsection{Estimation Results for Both Boundary Conditions Combined (Case 4)}

Since the insulated boundary cases were giving questionable results for emissivity estimates, a final parameter estimation case was examined. This case used both specified temperature boundary data and information from the heated surface in the insulated boundary data in the objective function. Note that data was taken from two different experiments. It was hoped that by combining both boundary conditions that the effects of noise and random errors would be reduced, resulting in more consistent estimates. Graphical and tabular results are shown in Figures 6.31-33 and Table 6.9 respectively. The emissivity estimates are almost the same as the one sensor insulated case with slightly smaller confidence intervals. Conduction area estimates are higher in magnitude than previous cases. Volumetric heat capacity estimates initially have small confidence intervals and are in the 0.3-0.4 range. At higher temperatures they suddenly become large and most likely incorrect, as this behavior deviates greatly from all other observed cases. 


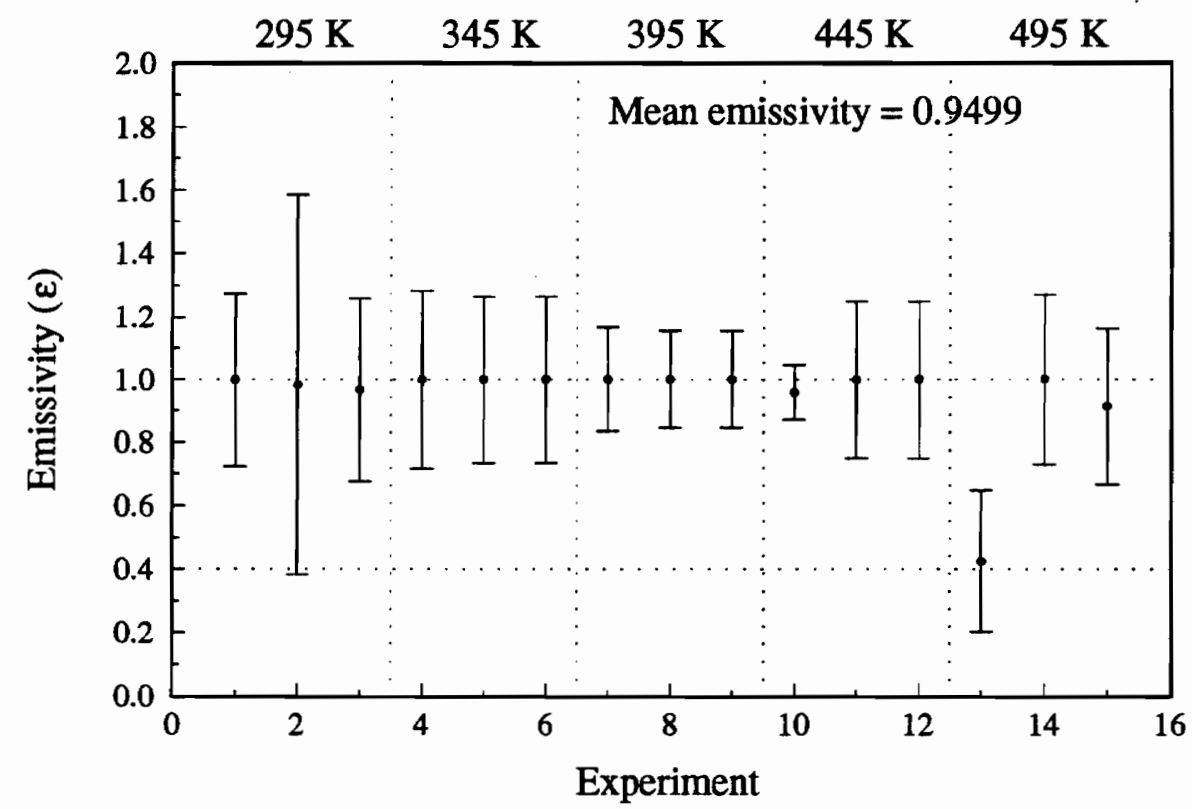

Figure 6.31 Emissivity Estimates for Both Boundary Conditions

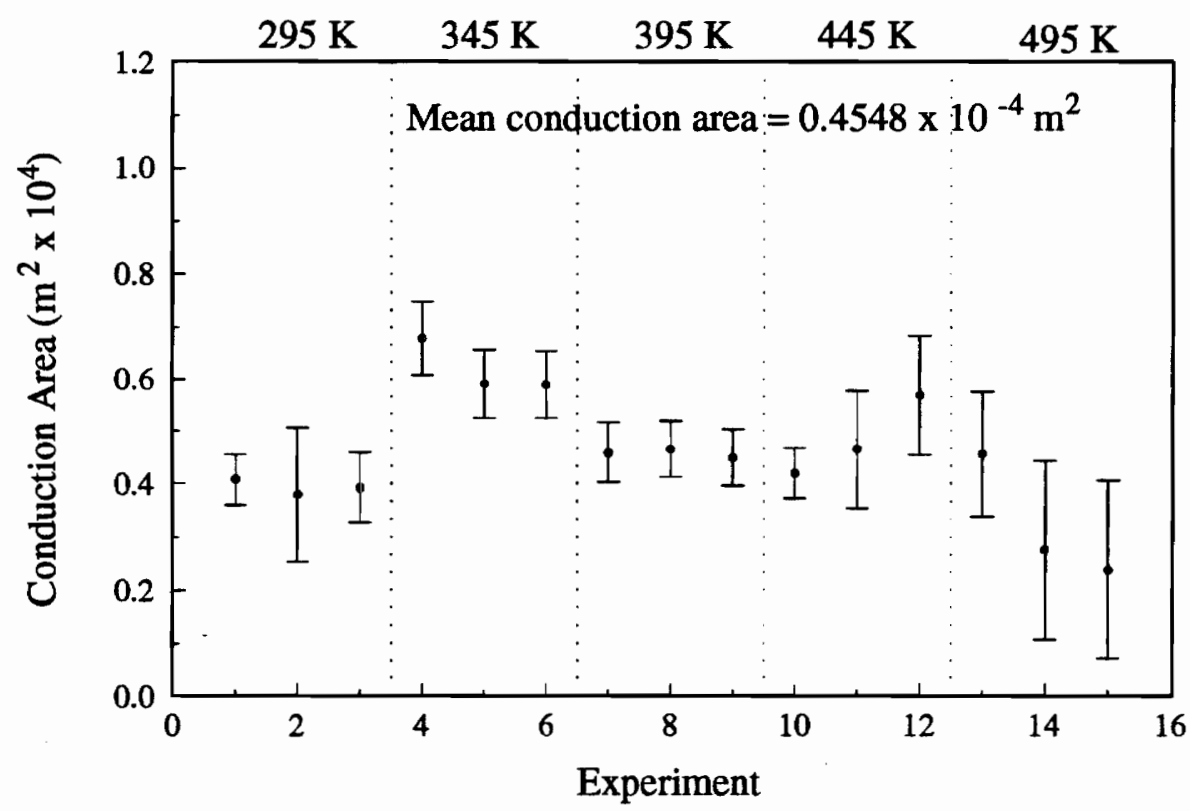

Figure 6.32 Conduction Area Estimates for Both Boundary Conditions 


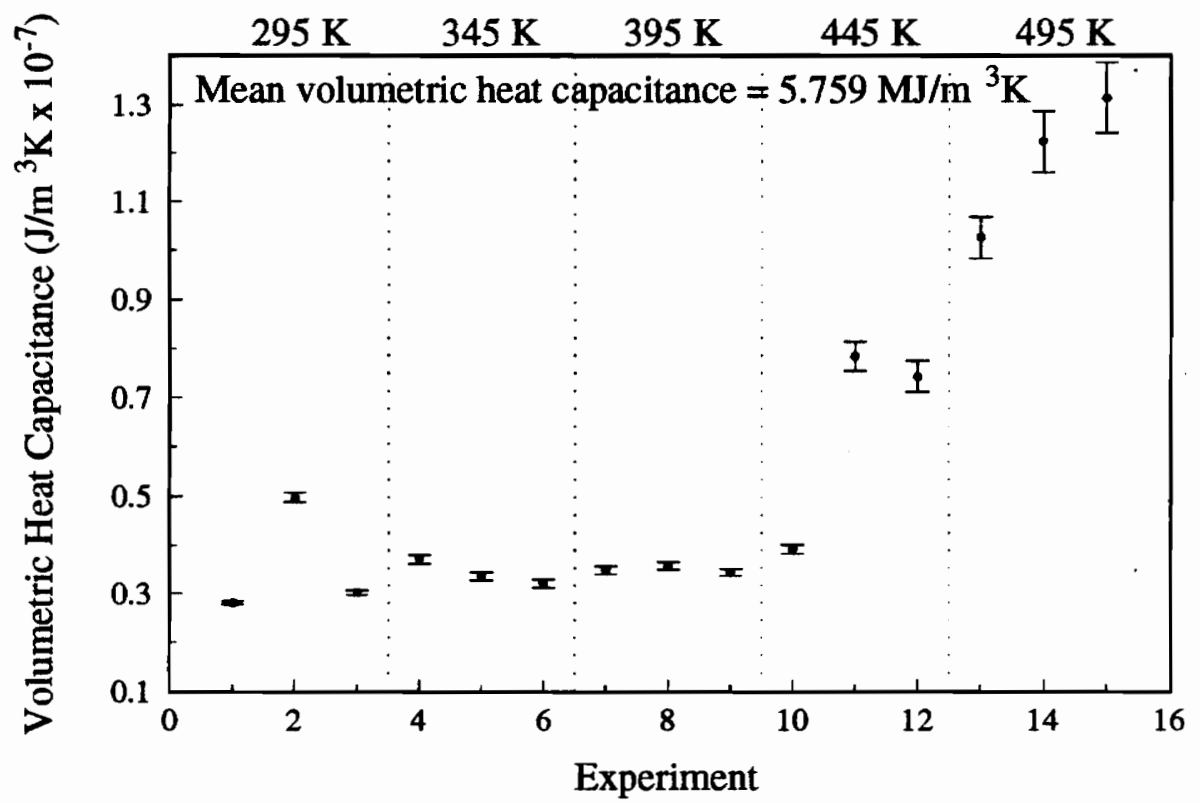

Figure 6.33 Volumetric Heat Capacity Estimates for Both Boundary Conditions 
Table 6.9 Mean Estimate Values at all Initial Temperatures for Estimation With Both Boundary Conditions

\begin{tabular}{||l||l||}
\hline Parameter & Estimate Value \\
\hline \hline Emissivity & $0.9499 \pm 0.0814$ \\
\hline Conduction Area & $0.4548 \pm 0.0648 \times 10^{-4} \mathrm{~m}^{2}$ \\
\hline Volumetric Heat Capacity & $0.5759 \pm 0.1963 \times 10^{7} \mathrm{~J} / \mathrm{m}^{3} \mathrm{~K}$ \\
\hline
\end{tabular}

\subsubsection{Comparison of Estimated Property Values}

The emissivity values for the four cases were very dependent on the model being used. Emissivity estimates had the widest confidence intervals of the three parameters being estimated. In three of the cases the values ranged from 0.7 to 1.0 while the insulated two sensor case converged to values near 0.4 . Only the specified temperature boundary case produced estimates which were not almost entirely on a constraint boundary.

Conduction area estimates are generally scattered in the 0.3 to $0.5 \times 10^{-4} \mathrm{~m}^{2}$ range, with no one model producing results which appear significantly different from the rest. The insulated boundary condition estimates have small confidence intervals which generally do not overlap. The specified temperature boundary results seem to have the least amount of variability between estimates.

The specified temperature boundary case again looks to provide the least variability in the volumetric heat capacity estimates. There is a wide range in the estimates for the insulated two sensor case. The combined boundary condition case appears to give reasonable results for the lower temperatures but not for $445 \mathrm{~K}$ or $495 \mathrm{~K}$. 


\subsection{Statistical Interpretation of Results}

Visual inspection of results revealed only general trends about the property estimates. A statistical comparison will reveal whether the estimates resulting from each of the four models are actually different and whether the initial temperature is has any effect on the results. If any effect, model or temperature, is found to be significant then multiple comparisons can be made. The statistical analysis performed on these results was done in conjunction with the Statistical Consulting Center at Virginia Polytechnic Institute and State University.

In order to assess the effects of the estimation model and initial temperature, an analysis of variance (ANOVA) was performed. The purpose of an analysis of variance is to determine whether or not differences exist between more than two treatments in an experiment. In this case the treatments would be the individual initial temperatures or the estimation models, with the set of all initial temperatures or set of all models being called factors. A set of observations for a specific initial temperature and model is called a cell. Each cell contains three observations in this study.

Sums of squares are computed for differences between treatment means, cell means, and individual observation means with the grand mean. These sums of squares are divided by the number of degrees of freedom resulting from their calculation to obtain mean square (MS) values. Ratios of these MS values are compared to the $F$ distribution with the same degrees of freedom to determine if treatment means are the same. If the observed $\mathrm{F}$ is greater than the $\mathrm{F}$ taken from the distribution then, $\mathrm{a}$ treatment is considered to be significant, as at least one treatment mean has been determined to be different from the others. 
In this study a two factor fixed model is being considered, where the factors and temperature and estimation model. The factors are considered to be fixed because they represent actual changes as opposed to different random samples. The three tests which the ANOVA will preform are to determine whether either factor or some interaction of the factors is significant. Interaction implies that the factors are not independent of each other and that the response will be dependent on the particular combination of both factors.

One analysis was performed for each parameter. Note that since all three parameter estimates originate from the same estimation procedure they are correlated in a statistical sense, meaning the assumption of homoscedasticity would be invalid. A multivariate analysis of variance (MANOVA) would be more appropriate; however, it is beyond the scope of this study. Instead, the confidence intervals obtained from the estimation procedure can be used as weight factors. The inverse square of these values is used to weight each individual observation when computing the sum of squares. This permits three separate analyses. The results are shown in Table 6.10.

In Table 6.10, DF represents degrees of freedom, $S S$, sum of squares, $M S$, mean square, $F$, the $F$ statistic, and $P r$, the probability of the critical $F$ being greater than the actual $F$. Since both factors are considered fixed, $F$ values are calculated by dividing the mean square value for each source by the mean square error value. The mean square for error (MSE) represents errors between individual observations and the grand mean. 
Table 6.10 Analysis of Variance for Estimated Parameters

\begin{tabular}{||l||l|l|l|l|l|l||}
\hline \hline Parameter & Source & DF & SS & MS & F Value & Pr $>$ F \\
\hline \multirow{4}{*}{ Emissivity } & model & 3 & 6.9512 & 2.3171 & 14.55 & 0.0001 \\
\cline { 2 - 8 } & init. temp. & 4 & 3.5218 & 0.8804 & 5.53 & 0.0012 \\
\cline { 2 - 8 } & interaction & 12 & 14.4730 & 1.2061 & 7.57 & 0.0001 \\
\hline \multirow{3}{*}{$\begin{array}{l}\text { Conduction } \\
\text { Area }\end{array}$} & model & 3 & 5.9980 & 1.9993 & 0.28 & .8418 \\
\cline { 2 - 8 } & init. temp. & 4 & 25.2952 & 6.3238 & 0.88 & .4872 \\
\cline { 2 - 8 } & interaction & 12 & 68.9597 & 5.7466 & 0.8 & .6525 \\
\hline \multirow{2}{*}{$\begin{array}{l}\text { Volumeat } \\
\text { Capacity }\end{array}$} & model & 3 & 987.3677 & 329.1226 & 5.35 & 0.0034 \\
\cline { 2 - 7 } & init. temp. & 4 & 713.1033 & 178.2758 & 2.9 & 0.0338 \\
\hline
\end{tabular}

The above table shows that the model, initial temperature, and interaction are all significant factors for emissivity and volumetric heat capacity as the $p$ values (shown in bold) are all less than the significance level of 0.05 . It also shows that no factors are significant for conduction area. This means that all models and all initial temperatures will result in the same area estimates. Concerning the other two parameters, it is apparent that the $F$ values for the model are much higher than for initial temperature or interaction. The choice of the estimation model is therefore the most important characteristic. The presence of interaction is somewhat unexpected and difficult to explain. It indicates that different models yielded different estimates at different initial temperatures. The cause could be due to experimental error (possibly the insulated boundary) or numerical ill-conditioning in the estimation routine.

Multiple comparison tests are useful in determining which members of a treatment are statistically different. Since no significance was found for the conduction area estimates no multiple comparisons can be made. For the other two parameters, the presence of interaction complicates multiple comparison tests. It is necessary to fix one 
treatment (initial temperature) and compare the model at that level. A studentized range test (HSD) was performed on the models for emissivity and volumetric heat capacity. This test ranks the cell means in order from largest to smallest. Orthogonal comparisons are made between the largest and each succeeding smaller cell mean. The orthogonal condition scales the cell means by an orthogonal set of numbers which will ensure that all comparisons are independent. If a simple $t$-test were performed for each possible pair of models then the probability of obtaining an incorrect result, even at the $95 \%$ confidence level would be significant. The cell means are compared by effectively determining whether or not their difference is greater than a value which is proportional to the square root of MSE and the studentized range distribution. This interval is wider than for a standard $t$-test.

The results of these comparisons indicate that only the emissivity estimates from the insulated boundary case with two sensors is significantly different from the others. The volumetric heat capacity estimates were not shown to be significantly different. This is not what was demonstrated by the original analysis of variance. The estimates are believed to be different although the method is too general or flexible to be able to determine the exact pair.

\subsection{High Temperature Numerical Experiments}

High temperature numerical experiments were run to determine if increasing the initial temperature would boost the amount of heat transferred by radiation and produce more accurate emissivity estimates. Recall that no actual data was taken over the starting temperature of $495 \mathrm{~K}$ for fear of damaging the Kapton coated RTDs and heater. 
Temperature histories were generated using the same values of parameters that were used in the experimental design. Starting temperatures of $695 \mathrm{~K}$ and $895 \mathrm{~K}$ were examined. No higher temperatures were examined due to a lack of property data on $\mathrm{Ti}$ $6 \mathrm{Al}-4 \mathrm{~V}$ at these temperatures.

Random errors were added to the simulated data to represent measurement noise. The specified temperature boundary was investigated at $695 \mathrm{~K}$ with added random measurement noise of $\pm 0.5 \mathrm{~K}$ from the $695 \mathrm{~K}$ basis. Three numerical experiments were run. Table 6.11 shows the result of these simulations.

Recall that the initial values of the parameters were $\varepsilon=0.7, \mathrm{~A}=0.35 \times 10^{-4} \mathrm{~m}^{2}$, and $\rho C=0.27 \times 10^{7}$. Table 6.11 shows that the correct values can be recovered; however, measurement noise will induce wide confidence intervals at the higher temperatures.

The same procedure was repeated for the insulated boundary case using information from two sensors. The outcome was similar to that obtained by using real data. The estimates varied greatly and had very wide confidence intervals. Emissivity values were either at the 1.0 or 0.4 constraint. Even the other two parameters showed large fluctuations.

Table 6.11 Numerical Experiment at $695 \mathrm{~K}$ for the Specified Temperature Boundary

\begin{tabular}{|c||l|l|l||}
\hline \hline Experiment & Emissivity & $\begin{array}{c}\text { Conduction Area } \\
\left(\mathrm{m}^{2} \times 10^{4}\right)\end{array}$ & $\begin{array}{l}\text { Vol. Heat Cap. } \\
\left(\mathrm{J} / \mathrm{m}^{3} \mathrm{~K} \times 10^{-7}\right)\end{array}$ \\
\hline \hline 1 & $0.6277 \pm 0.4188$ & $0.4388 \pm 0.5019$ & $0.2646 \pm 0.0575$ \\
\hline 2 & $0.6895 \pm 0.3970$ & $0.3644 \pm 0.4740$ & $0.2567 \pm 0.0531$ \\
\hline 3 & $0.6668 \pm 0.4126$ & $0.3905 \pm 0.4930$ & $0.2626 \pm 0.0557$ \\
\hline
\end{tabular}


Two more cases were examined for this boundary condition. In one the starting temperature was raised to $895 \mathrm{~K}$ with random noise of $\pm 0.5 \mathrm{~K}$. The resulting emissivity values ranged from 0.43 to 0.91 but had an average value of 0.6552 . The confidence intervals for this case were on the order of 0.24 to 0.56 . Conduction area estimates were generally lower than the actual values. Volumetric heat capacity estimates remained nearly the same. The final numerical experiment involved the same boundary condition at $695 \mathrm{~K}$ with random noise of $\pm 0.1 \mathrm{~K}$. Little difference was observed between this case and the $\pm 0.5 \mathrm{~K}$ random noise case. The confidence intervals were marginally smaller, but not appreciably.

\subsection{Confidence Regions}

As stated in Chapter 3, errors in estimated parameters can best be shown by their confidence regions. The formulation for the confidence interval is given by Beck and Arnold to be

$$
(b-\beta)^{T}(P)^{-1}(b-\beta)=p s^{2} F_{1-\alpha}(p, n-p)
$$

where $\boldsymbol{b}-\boldsymbol{\beta}$ is a vector of parameter errors. In this case $p$, the number of parameters, is three. The number of measurements, $n$, is very large and can be considered infinity. The value of the $F$ statistic, $F_{0.05(3, \infty)}$ is 7.8. A plot of this quadratic function gives an ellipsoid for three parameters.

Figure 6.34 shows one half of the confidence region for the insulated boundary (two sensors) at $495 \mathrm{~K}$. Figure 6.35 shows one half of the confidence region for the 


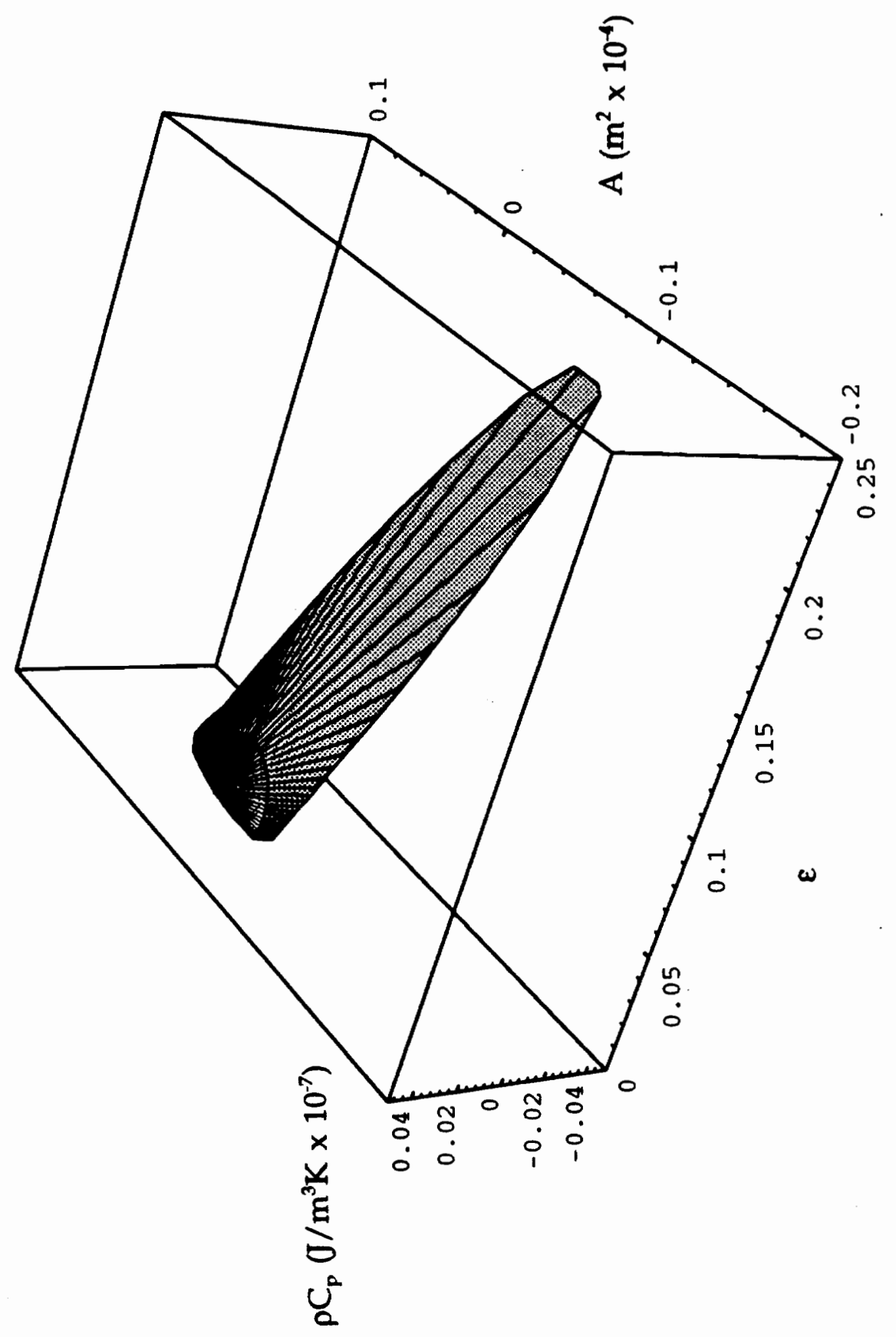

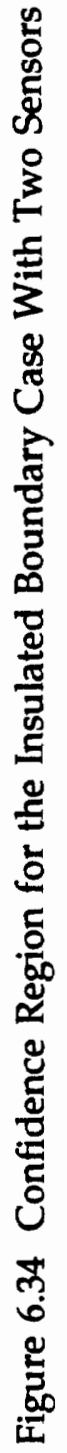




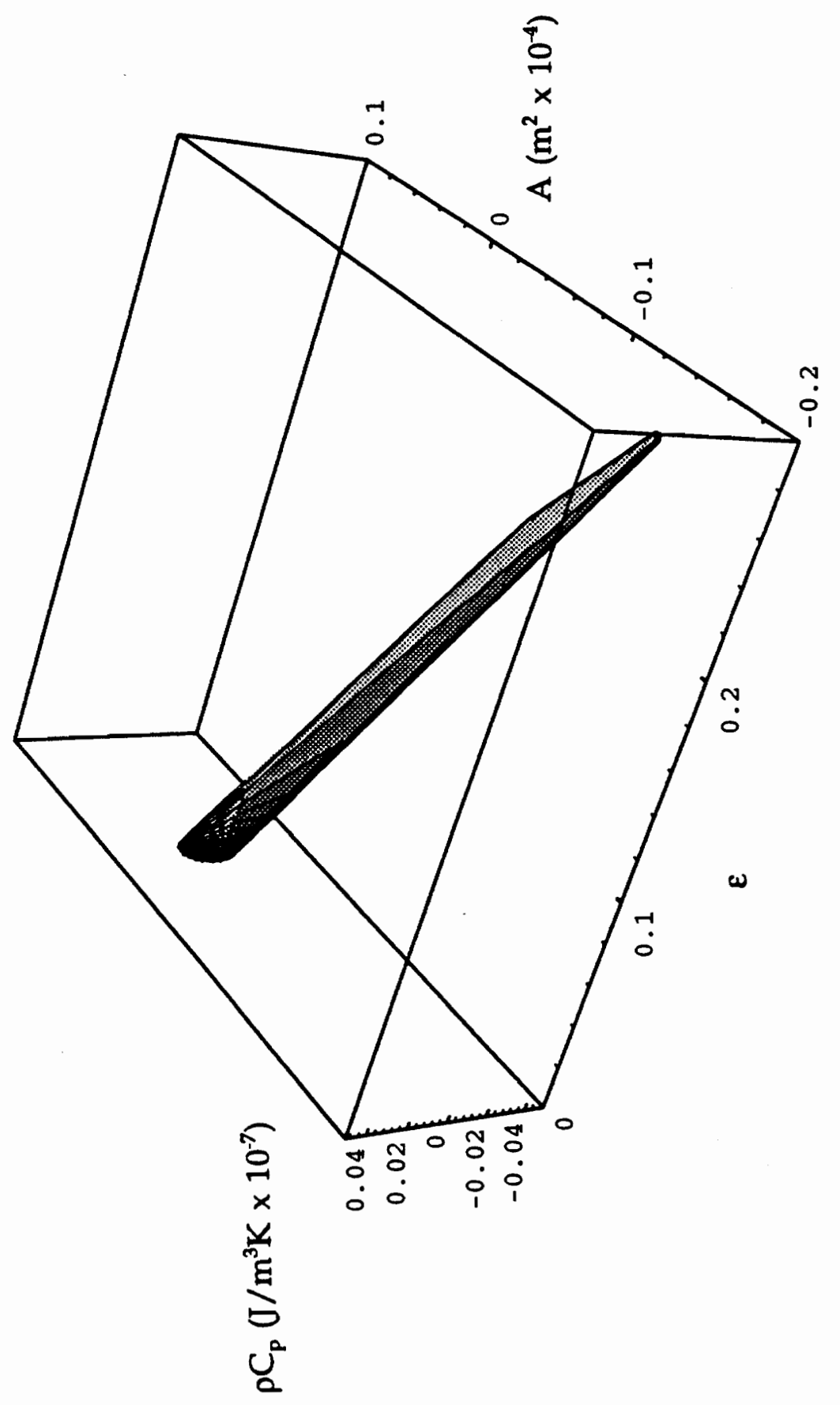

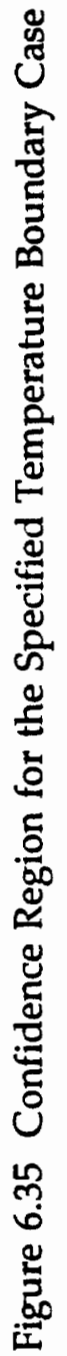


specified temperature boundary case at $495 \mathrm{~K}$. These ellipsoids are much longer in the direction corresponding to errors in emissivity than they are in the other two directions. This graphical representation is useful in determining what the errors may be in two parameters if the third is known. Note that the endpoints of these ellipsoids do not exactly correspond to the lengths suggested by the confidence intervals. Both are approximations and based on different statistical distributions. Also note that both are oblique. This signifies correlation among the parameters. Uncorrelated parameters would have confidence regions perfectly aligned with the coordinate axes.

\subsection{Heat Transfer Modes in the Structure}

In order to better understand the magnitudes of conduction and radiation heat transfer in the structure, a program was developed to calculate the heat transferred by each mode. Figure 6.36 shows the amount of heat transferred by conduction and radiation as the structure is being heated both at $295 \mathrm{~K}$ and $495 \mathrm{~K}$. In both cases the two modes add together to give about $350 \mathrm{~W} / \mathrm{m}^{2}$, the magnitude of the applied heat flux. The radiant heat flux increases with time as the temperature gradient across the structure becomes greater. It increases by about $55 \%$ at steady state when the starting temperature is increased from $295 \mathrm{~K}$ to $495 \mathrm{~K}$. Figure 6.37 shows heat transfer in the insulated boundary case. While the shape of these curves looks the same as before, the conduction heat transfer dominates due to the small temperature gradient. It is expected that increasing the temperature gradient or the starting temperature would increase the radiant heat flux. There is a competing effect. The thermal conductivity of the core increases from $7.5 \mathrm{~W} / \mathrm{mK}$ at $295 \mathrm{~K}$ to $10.7 \mathrm{~W} / \mathrm{mK}$ at $495 \mathrm{~K}$, and increases slightly faster 


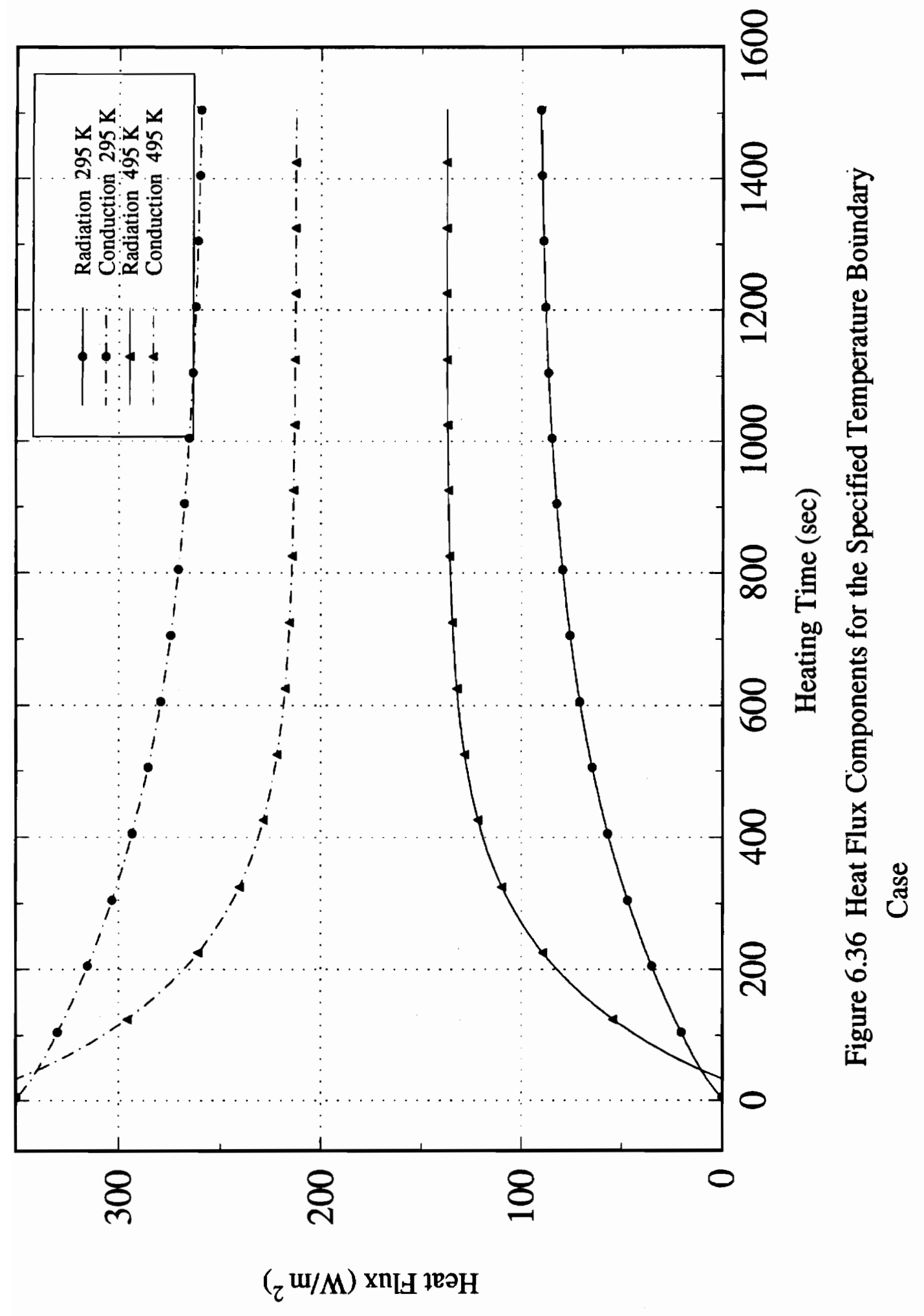




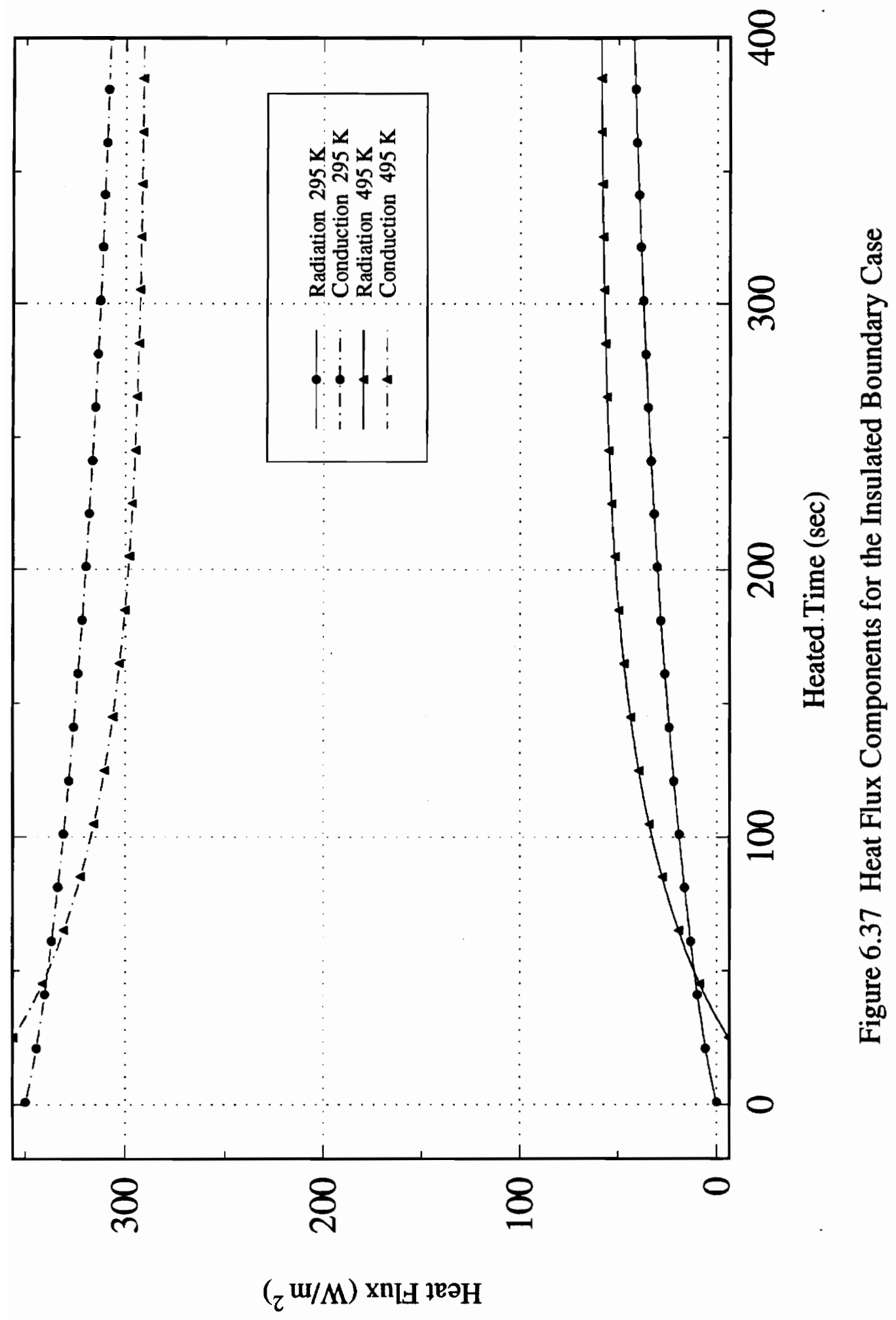


at even higher temperatures. The increasing thermal conductivity would somewhat suppress radiation.

\subsection{RMS Error Calculations}

The thermal properties which are estimated will later be used in the calculation of temperature distributions for thermal stress analyses. One measure of the errors in estimated thermal properties is the Root Mean Square (RMS) error they would produce when compared to the temperature histories from which they were estimated. The RMS error is defined as

$$
R M S=\sqrt{\frac{\sum_{i=1}^{N} \sum_{j=1}^{M}\left(Y_{i j}-T_{i j}\right)^{2}}{N M}}
$$

where $Y_{i j}$ and $T_{i j}$ are the measured and calculated temperatures, respectively, at time $i$ and for sensor $j$. The total number of temperatures measurements is donated by $N$, and the total number of sensors is $M$. Note that for the specified temperature boundary case $M$ is one, and for the insulated boundary case it is two.

RMS error calculations were made in two different ways. A measured temperature data set was first compared to calculated values generated by using the parameter estimates generated by that particular experiment. These values are given as $\mathrm{RMS}_{\mathrm{I}}$. The mean values for a model at all temperatures were also used to calculate RMS error values. Here, mean estimates were used to calculate temperature histories that were compared to individual data sets. These values are indicated by $\mathrm{RMS}_{\mathrm{M}}$. A summary of the RMS calculations are shown in Table 6.12 . 
Table 6.12 Average RMS Error Calculations for Specified Temperature and Insulated (Two Sensor) Boundaries.

\begin{tabular}{||c|c|c|c|c||}
\cline { 2 - 5 } & & Specified & \multicolumn{1}{c|}{ Temperature } & \multicolumn{2}{c||}{ Insulated } & 2 Sensor \\
\cline { 2 - 5 } & $295 \mathrm{~K}$ & $495 \mathrm{~K}$ & $295 \mathrm{~K}$ & $495 \mathrm{~K}$ \\
\hline \hline $\begin{array}{r}\mathrm{RMS}_{\mathrm{I}} \text { (K) } \\
\text { \%aximum }\end{array}$ & 0.3826 & 0.3541 & 1.5589 & 3.4035 \\
Temp. Rise & $1.28 \%$ & $2.36 \%$ & $9.17 \%$ & $30.94 \%$ \\
\hline \hline $\begin{array}{r}\text { RMS } \\
\text { \% }(\mathrm{K})\end{array}$ & 0.8893 & 2.0803 & 1.3373 & 3.0944 \\
Temp. Rise & $2.96 \%$ & $13.87 \%$ & $7.87 \%$ & $28.13 \%$ \\
\hline
\end{tabular}

Even though it has been determined that temperature should not have a significant effect on the estimated properties, it is clear that smaller RMS errors occur at $295 \mathrm{~K}$ than at $495 \mathrm{~K}$ for both boundary conditions. It is also apparent that the specified temperature boundary estimation gives better results than the insulated boundary condition. The specified temperature boundary errors are smaller in magnitude and as a percent of maximum temperature rise in the experiment. Also note that the $\mathrm{RMS}_{\mathrm{M}}$ values for the insulated boundary condition are slightly larger than the $R M S_{1}$ values, which is the opposite of what is expected.

Another question which arises is how errors in the estimated parameters would affect temperature histories calculated from these parameters. RMS errors were calculated for the specified temperature boundary condition using the average values from Table 6.6 for two parameters and the high and low end of the other parameter's estimated confidence interval. An average of these values is shown in Table 6.13. 
Table 6.13 Mean RMS Temperature Errors Corresponding to Parameter Confidence Intervals for the Specified Temperature Boundary Condition

\begin{tabular}{||l|l||c|c||}
\cline { 2 - 4 } \multicolumn{1}{c|}{} & RMS Error (K) & \% Max. Temp. Rise \\
\hline \multirow{2}{*}{ Emissivity } & high & 2.3404 & $11.70 \%$ \\
\cline { 2 - 4 } & low & 0.8841 & $4.42 \%$ \\
\hline \multirow{2}{*}{$\begin{array}{l}\text { Conduction } \\
\text { Area }\end{array}$} & high & 3.5478 & $17.74 \%$ \\
\cline { 2 - 4 } $\begin{array}{l}\text { Face Sheet Heat } \\
\text { Capacity }\end{array}$ & low & 2.4514 & $12.26 \%$ \\
\cline { 2 - 4 } & high & 1.5473 & $7.74 \%$ \\
\hline \hline
\end{tabular}

Notice that the RMS errors are higher for parameters taken at the higher end of the confidence interval. This implies that if a parameter estimate has been estimated too large, then greater temperatures errors would result if these parameters were used in a thermal analysis. This table also shows that temperature errors can most easily arise from errors in conduction area. The emissivity estimates showed a great deal of variability among estimation models. RMS errors are much smaller for smaller values of emissivity, meaning that actual estimate values probably occur in the lower end of the confidence interval. Therefore, if an estimate appears to be too high it might be wise use a slightly lower value. 


\section{CHAPTER 7}

\section{Summary and Conclusions}

There were four goals set forth at the beginning of this research in relation to the estimation of the thermal properties of honeycomb core sandwich structures. These included the development of a heat transfer model for the structure, experimental design, implementation of a parameter estimation procedure, and the estimation of all pertinent thermal properties. The work was based on previous studies by Moncman (1994) and Hanak (1995), but with extensive modifications.

\subsection{Heat Transfer Model}

The first objective was to determine how to model the heat transfer through the structure. A thermal image analysis proved that a one dimensional approximation would be sufficient. This simplification was made possible because the thermal conductivity of the face sheets was much higher than the core material. The face sheet was modelled as a lumped capacitance, which was estimated. Since the conductive properties of the core material were well known, it was decided to estimate the effective area of core. The 
emissivity of the core was also estimated.

\subsection{Experimental Design}

Optimum heating times for the specified temperature boundary varied from 4560 sec for $295 \mathrm{~K}$ to $1500 \mathrm{sec}$ for $495 \mathrm{~K}$ using the D-criterion. The scaled confidence interval approach reduced these times to a range of $3000 \mathrm{sec}$ to $1500 \mathrm{sec}$. Heating times for the insulated case ranged from 400 to 300 seconds, as given by the scaled confidence interval approach. The optimum total experiment time varied from $4600 \mathrm{sec}$ at $295 \mathrm{~K}$ to $2500 \mathrm{sec}$ at $495 \mathrm{~K}$ for the specified temperature boundary condition. The insulated boundary times went from $1630 \mathrm{sec}$ to $1400 \mathrm{sec}$ as temperature increased from $295 \mathrm{~K}$ to $495 \mathrm{~K}$.

\subsection{Parameter Estimation Algorithm}

The first attempt at estimating parameters from the collected data was done using the Box-Kanemasu modification of the Gauss method. The oscillatory nature of the BoxKanemasu method showed the need for a method which allowed for constrained minimization. A penalty function approach was used in this study. Inequality constraints were placed on all parameters. Conduction area and face sheet capacitance were constrained to within a factor of ten of the original estimates for these parameters. Emissivity was bounded at 1.0 on the high end, as this is the highest possible value by definition. It was bounded at 0.4 on the low end. 


\subsection{Parameter Estimates}

The specified temperature boundary condition (Case 1) yielded the following results:

$\varepsilon=0.8820 \pm 0.3098, A=0.4178 \pm 0.1428 \times 10^{-4} \mathrm{~m}^{2}, \rho C_{p}=0.2990 \pm 0.0150 \times 10^{7} \mathrm{~J} / \mathrm{m}^{3} \mathrm{~K}$

The insulated boundary estimation using information in the objective function from both sensors (Case 2) had the following results:

$\varepsilon=0.4985 \pm 0.3791, \quad A=0.3905 \pm 0.0794 \times 10^{-4} \mathrm{~m}^{2}, \rho C_{p}=0.3721 \pm 0.3333 \times 10^{7} \mathrm{~J} / \mathrm{m}^{3} \mathrm{~K}$

The insulated boundary condition using information from the heated surface in the objective function and specifying the temperature at the other boundary (Case 3) yielded these estimates:

$\varepsilon=0.9333 \pm 0.8555, A=0.5760 \pm 0.0979 \times 10^{-4} \mathrm{~m}^{2}, \rho C_{p}=0.2628 \pm 0.0129 \times 10^{7} \mathrm{~J} / \mathrm{m}^{3} \mathrm{~K}$

It was hoped that the insulated boundary data could be salvaged by using it in conjunction with a specified temperature data set (Case 4). The results are as follows:

$\varepsilon=0.9499 \pm 0.2522, \quad A=0.4548 \pm 0.0889 \times 10^{-4} \mathrm{~m}^{2}, \rho C_{p}=0.5759 \pm 0.2120 \times 10^{7} \mathrm{~J} / \mathrm{m}^{3} \mathrm{~K}$ 


\subsection{Conclusions}

The following five conclusions can be drawn from this study:

1) A one dimensional model is sufficient to model heat transfer through the structure.

2) The scaled confidence interval approach showed that experimental times could be decreased from what the D-optimal criterion predicted with little change in the accuracy of estimated parameters, especially at lower temperatures.

3) Constrained optimization techniques, such as the Penalty Function Method, can be used used to solve parameter estimation problems in which correlation exists which would prevent unconstrained methods from converging.

4) The specified temperature boundary produced the most reliable parameter estimates because it had lower RMS temperature errors, the conduction area estimate was close to the measured value, and the emissivity estimates were not all located at a constraint boundary.

5) Estimation cases involving insulated boundary data produced varied results due to the unknown initial state of the boundary insulation. The use of insulated boundaries at sensor locations is not practical for transient experiments. 


\section{CHAPTER 8}

\section{Recommendations}

The methods and experiments used in study were by no means exhaustive. Other experimental designs could be used, other models tried, or different parameters estimated. The following recommendations are made to improve and broaden the scope of this study.

While the method of experimental design and thermal property estimation using the specified temperature boundary were largely successful, use of the insulated boundary condition was not. Use of this boundary condition is not recommended for two reasons. First, it takes a long time for the insulation to reach steady state when operating at different initial temperatures. Second, the smaller temperature gradients produced by this boundary condition do not produce very sensitive information for parameter estimation. Due to the length of the experiment, the insulation may also act as a heat sink.

In order to increase parameter sensitivity, it is recommended that a specified temperature type boundary condition be investigated where large temperature gradients can be maintained. This would require something more 
powerful than the electric resistance heater used in this experiment. Since higher sensitivities were found at lower temperatures, one boundary should be maintained near room temperature.

Estimation of multidimensional properties, i.e. thermal conductivities, would be difficult but possible. It is unclear if the use of thermocouples would be possible due to due the metallic nature of the sandwich structure. If large test samples were available then small RTDs could possibly be used as temperature sensors. Any samples which would be used for this estimation should be much larger in size than the unit cell dimensions in order to eliminate localized effects from cell walls. If a three dimensional analytical model were developed, it could be used to determine how much error resulted from using the one dimensional conduction approximation. A larger number of radiation view factors could also be used.

Concerning the estimation method, it would be desirable to investigate the use of another routine, such as a direct search. This would assess the effects of the constraints on the penalty function algorithm. It would also determine whether or not the inconsistent estimates resulting from the insulated boundary condition were due to experimental errors and uncertainties in the physical model or the method itself. sandwich structure was to be able to perform a thermal stress analysis. Since 
thermal and mechanical stresses both occur in actual operating conditions, it is necessary to know both the thermal and mechanical properties of the structure. Temperature dependent mechanical properties could be estimated. This would involve running an experiment where a sample was thermally and mechanically loaded at the same time, as well as instrumented with RTDs and strain gages. EAL possesses the ability to perform a combined analysis. The existing parameter estimation routine could easily be modified to estimate the parameters. 


\section{BIBLIOGRAPHY}

Akay, M. and R. Hanna, 1990, "Comparison of Honeycomb Core and Foam Core Carbon Fibre/Epoxy Sandwich Panels," Composites, Vol. 24, No. 4, pp. 325-331.

Asako, Y., H. Nakamura and Z. Chen, 1990, "Three Dimensional Natural Convection in an Inclined Air Slot With a Hexagonal Honeycomb Core," AIAA/ASME Thermophysics and Heat Transfer Conference, ASME Heat Transfer Division, Vol. 140, pp. 19-24.

Bard, Y., 1974, Nonlinear Parameter Estimation, Academic Press, New York.

Bayard, D.S., Hadaegh, F.Y., and Meldrum, D.R., 1988, "Optimal Experiment Design for Identification of Large Space Structures," Automatica, Vol. 24, No. 3, pp. 357-364

Beck, J. V., 1966, "Transient Determination of Thermal Properties," Nuclear Engineering and Design, Vol. 3, pp. 373-381.

Beck, J. V., 1969, "Determination of Optimum, Transient Experiments for Thermal Contact Conductance," International Journal of Heat and Mass Transfer, Vol. 12, pp. 621-633.

Beck, J. V., and K. J. Arnold, 1977, Parameter Estimation in Engineering and Science, John Wiley \& Sons, New York.

Box, G. E. P. and H. Kanemasu, 1972, "Topics in Model Building, Part II, on Nonlinear Least Squares," Tech. Report No. 321, University of Wisconsin, Dept. of Statistics, Madison, Wisconsin.

Burr, I. W., 1974, Applied Statistical Methods, Academic Press, New York.

Chen, Q. and C. Levy, 1994, "Vibration Analysis of a Partially Covered Double Sandwich Cantilever Beam With Concentrated Mass at the Free End," Solids and Structures, Vol. 31, No. 17, pp. 2377-2391.

Cobelli, C. and A. Ruggeri, 1991 "A Reduced Sampling Schedule for Estimating the Parameters of the Glucose Minimal Model from a Labeled IVGTT," IEEE Transactions on Biomedical Engineering, Vol. 38, No. 10, pp. 1023-1029. 
Ezawa, Y. and N. Okamoto, 1995, "Development of Contact Stress Analysis Programs Using the Hybrid Method of FEM and BEM," Computers \& Structures, Vol. 57, No. 4. pp. 691-698.

Faldale, T. D., A. V. Nenarokomov, and A. F. Emery, 1995, "Uncertainties in Parameter Estimation: The Inverse Problem," International Journal of Heat and Mass Transfer, Vol. 38, No. 3, pp. 511-518.

Farghaly, S. H. and M. G. Shebl, 1992, "Vibration Characteristics of End Mass Loaded Undamped Sandwich Beams With Elastically Constrained Ends," Journal of Sound and Vibration, Vol. 159, No. 2, pp. 237-249.

Fuehne, J. P. and J. J. Engblom, 1992, "Finite Element/Penalty Function Method for Computing Stresses Near Debonds," AIAA Journal, Vol. 30 No. 6, pp. 1625-1631.

Haftka, R. T. and D. S. Malkus, 1981, "Calculation of Sensitivity Derivatives in Thermal Problems by Finite Differences", International Journal for Numerical Methods in Engineering, Vol. 17, pp. 1811-1821.

Hanak, J. P., 1995, Experimental Verification of Optimal Experimental Designs for the Estimation of Thermal Properties of Composite Materials, M.S. Thesis, Department of Mechanical Engineering, Virginia Polytechnic Institute and State University, Blacksburg, VA.

Hogg, R. V. and J. Ledolter, 1992, Applied Statistics for Engineers and Physical Scientists, Macmillan, New York.

Incropera, F. P. and D. P. Dewitt, 1985, Fundamentals of Heat and Mass Transfer, John Wiley \& Sons, New York.

Jamaluddin, A. S. and P. J. Smith, 1988, "Predicting Radiative Transfer in Axisymmetric Cylindrical Enclosures Using Discrete Ordinance Method," Combustion Science and Technology, Vol. 62, No. 4-6, pp. 173-186.

Kaushika, N. D., P. K. Sharma and R. Priya, 1992, "Solar Thermal Analysis of Honeycomb Roof Cover System for Energy Conservation in an Air - Conditioned Building, Energy and Buildings, Vol. 18, No. 1, pp. 45-49.

Kaushika, N. D., R. A. Ray, and P. Priya, 1990, "Honeycomb Solar Collector and Storage System," Energy Conversion and Management, Vol. 30, No. 2, pp. 127-134.

Loh, M. H. and J. V. Beck, 1991, "Simultaneous Estimation of Two Thermal Conductivity Components from Transient Two-Dimensional Experiments," ASME Paper No. 91-WA/HT-11 ASME, NY.

Maubourguet-Pellerin, M. M. and F. Pellerin, "Evaluation of Mean Heat Transfer Coefficients in Periodically Corrugated Channels," Numerical Heat Transfer, Vol. 11, No. 2, pp. 213-227. 
Moncman, D. A., 1994, Optimal Experimental Designs for the Estimation of Thermal Properties of Composite Materials, M.S. Thesis, Department of Mechanical Engineering, Virginia Polytechnic Institute and State University, Blacksburg, VA.

Moncman, D. A., J. P. Hanak, D. C. Copenhaver, and E. P. Scott, 1995, "Optimal Experimental Designs for Estimating Thermal Properties," Proceedings, 4th ASME/JSME Thermal Engineering Joint Conference, ASME.

Patankar, S. V., 1980, Numerical Heat Transfer and Fluid Flow, Hemisphere Publishing, New York.

Pfahl, R. C., and B. J. Mitchell, 1970, "Simultaneous Estimation of Six Thermal Properties of a Charring plastic," International Journal of Heat and Mass Transfer, Vol 13, pp. 275-286.

Rao, S. S., 1979, Optimization: Theory and Applications, Wiley Eastern Limited, New Dehli.

Saad, Z., and Scott E. P., 1994, "Estimation of Temperature Dependent Thermal Properties of Basic Food Solutions During Freezing," HTD - Fundamentals of Phase Change: Sublimation and Solidification, ASME, Vol. 268.

Scott, E. P., 1994, "Analysis of Honeycomb Structure," NASA Technical Report, Thermal Structures Branch.

Scott, L. A., and E. P. Scott, 1993, "Inverse and parameter estimation problems related to cryosurgery," HTD - Advansed in Bioheat and Mass Transfer: Microscale Analysis of Thermal Injury Process, Instrumentation, Modeling, and Clinical Applications, ASME, Vol. 268.

Scott, E. P. and J. V. Beck, 1992, "Estimation of Thermal Properties in Epoxy Matrix/Carbon Fiber Composite Material," Journal of Composite Materials, Vol. 26 No. 1, pp. 132-149.

Scott, E. P. and R.T. Haftka, 1995, "Optimization and Experiments," American Institute of Aeronautics and Astronautics, pp. 1-11.

Soria, J. and M. P. Norton, 1990, "Simple-to-Fabricate Composite Convective Heat Transfer Test Surface," Applied Energy, Vol. 35, No. 2, pp. 109-123.

Swann, R. T. and C. M. Pittman, 1961, "Analysis of Effective Thermal Conductivities of Honeycomb-Core and Corrugated-Core Sandwich Panels," NASA Technical Note D-174, NASA, Washington, D.C.

Taktak, R., E. P. Scott and J. V. Beck, 1991, "Optimal Experimental Designs for Estimating the Thermal Properties of Composite Materials," Proceedings of the 3rd ASME/JSME Thermal Engineering Joint Conference, ASME, Vol. 3, pp. 481-488. 
Teo, K.L., V. Rehbock, and L. S. Jennings, 1993, "New Computational Algorithm for Functional Inequality Constrained Optimization Problem," Automatica, Vol. 29, No. 3, pp. 789-792.

Touloukian, Y. S., et al., 1970, Thermophysical Properties of Matter, Vol. 1, 4, 7, IFI/Plenum, New York.

Vanderplaats, G. N., 1984, Numerical Optimization Techniques for Engineering Design: With Applications, McGraw-Hill, New York.

Whetstone, W. D. 1983, EISI-EAL Engineering Analysis Language, Engineering Information Systems, Inc., San Jose, CA.

Zeng, G. L. and G. T. Gullberg, 1993, "MAP Algorithm for Transmission Computed Tomography," Proceedings of the 1993 IEEE Nuclear Science Symposium and Medical Imaging Conference, Vol 2, pp. 1202-1204.

Zhang, X. and A. Atrens, 1994, "Evaluation of Unknown Parameters in the MeltSpinning Process," Journal of Materials Science, Vol. 29, No. 2, pp. 544-547. 


\section{APPENDIX A}

\section{Scaled Confidence Intervals and Eigenvalues}

This appendix contains graphs that were not shown in Chapter 6. These include scaled confidence intervals for the specified temperature boundary condition and the insulated boundary condition at $345 \mathrm{~K}, 395 \mathrm{~K}$, and $445 \mathrm{~K}$. These figures were used in determining optimum heating and total experiment times. Also shown are the eigenvalues of $\left|X^{T} X\right|$ used to determine optimum heating time. 


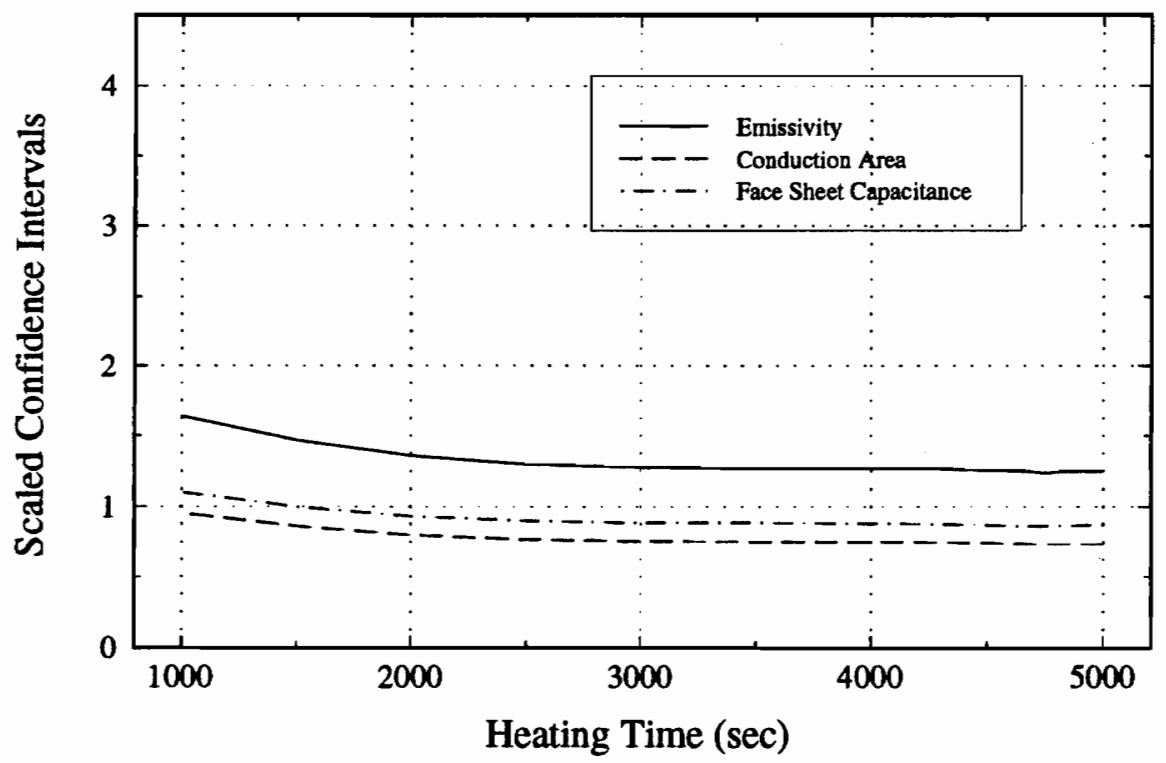

Figure A.1 Scaled Confidence Intervals at $345 \mathrm{~K}$ for the Specified Temperature Boundary Case, Used to Determine Heating Time

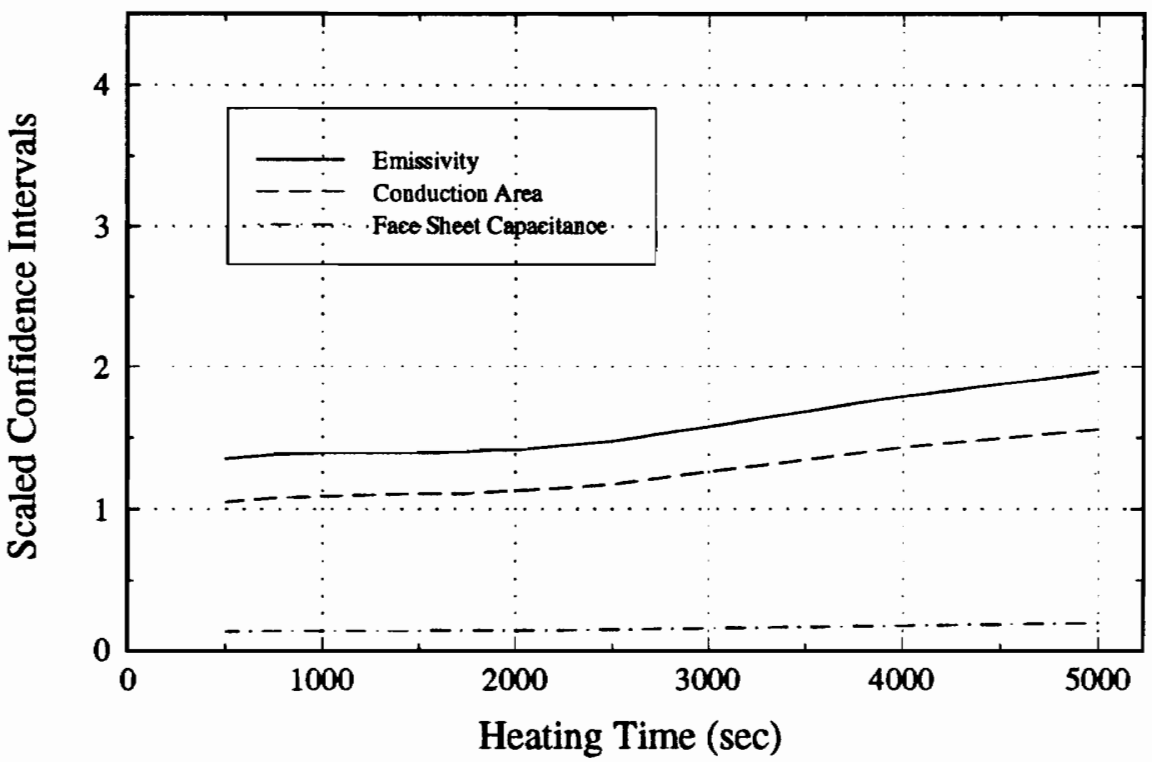

Figure A.2 Scaled Confidence Intervals at $395 \mathrm{~K}$ for the Specified Temperature Boundary Case, Used to Determine Heating Time 


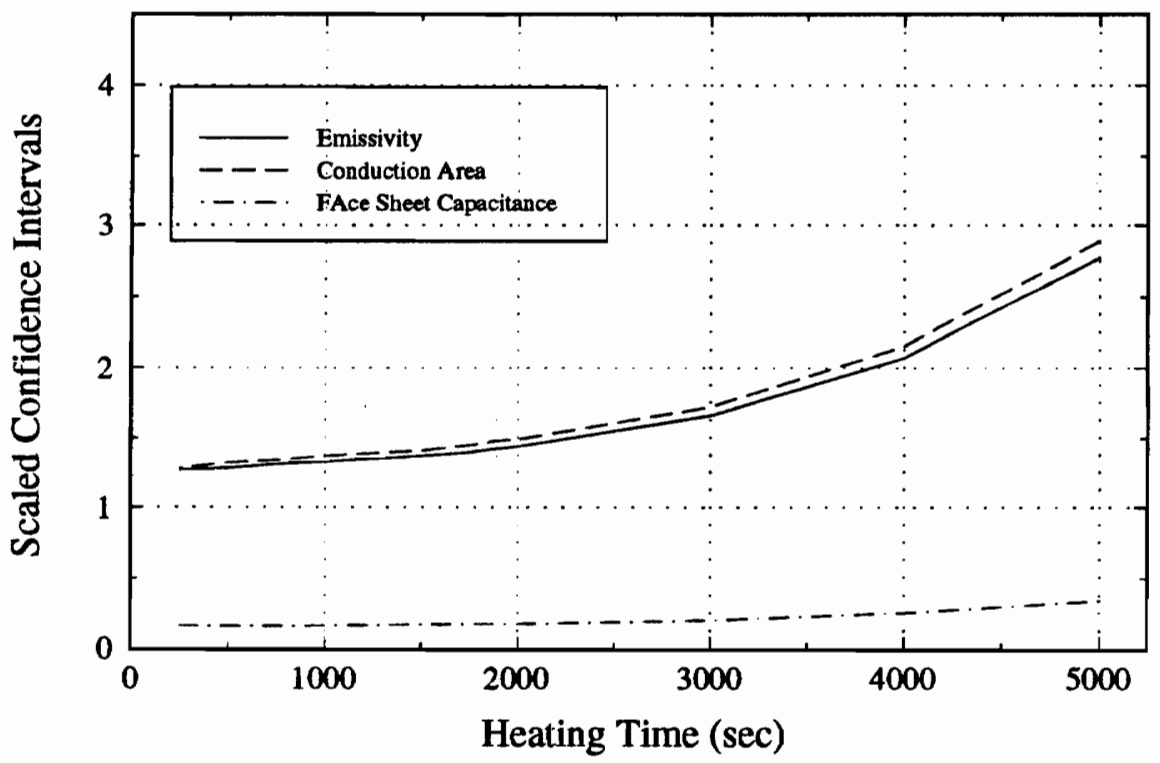

Figure A.3 Scaled Confidence Intervals at $445 \mathrm{~K}$ for the Specified Temperature Boundary Case, Used to Determine Heating Time

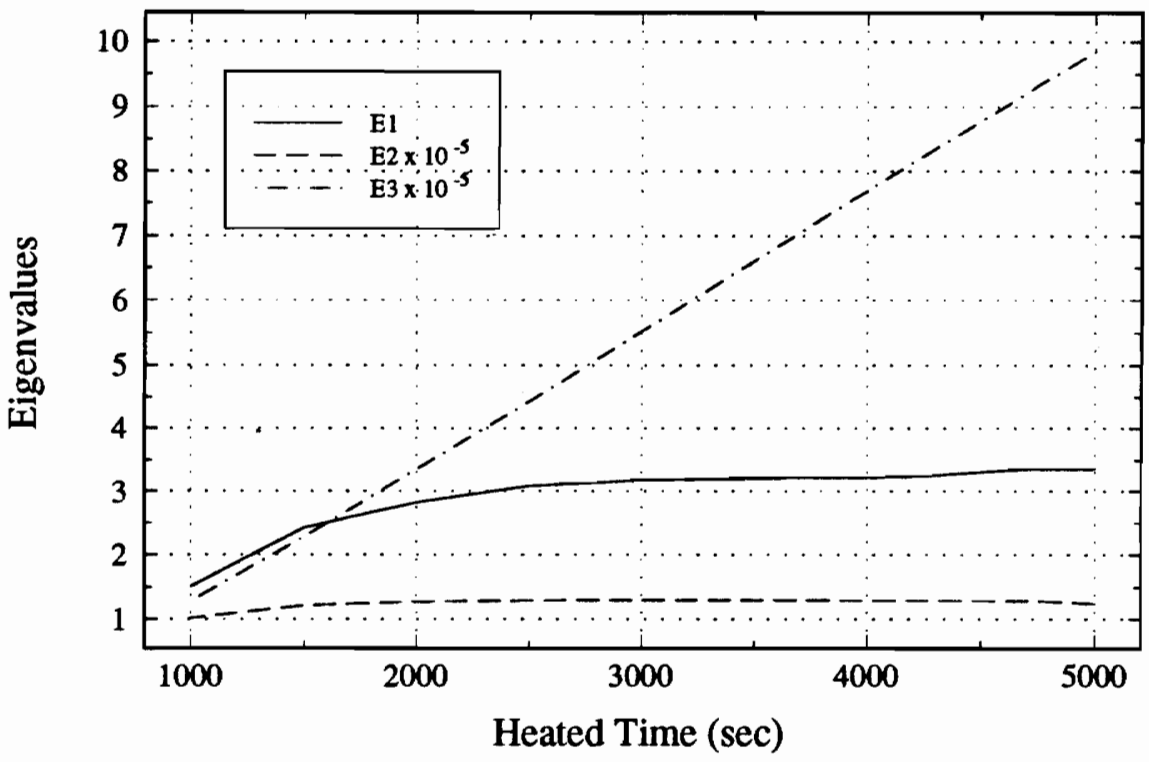

Figure A.4 Determinant Eigenvalues at $345 \mathrm{~K}$ for the Specified Temperature Boundary Case, Used to Determine Heating Time 


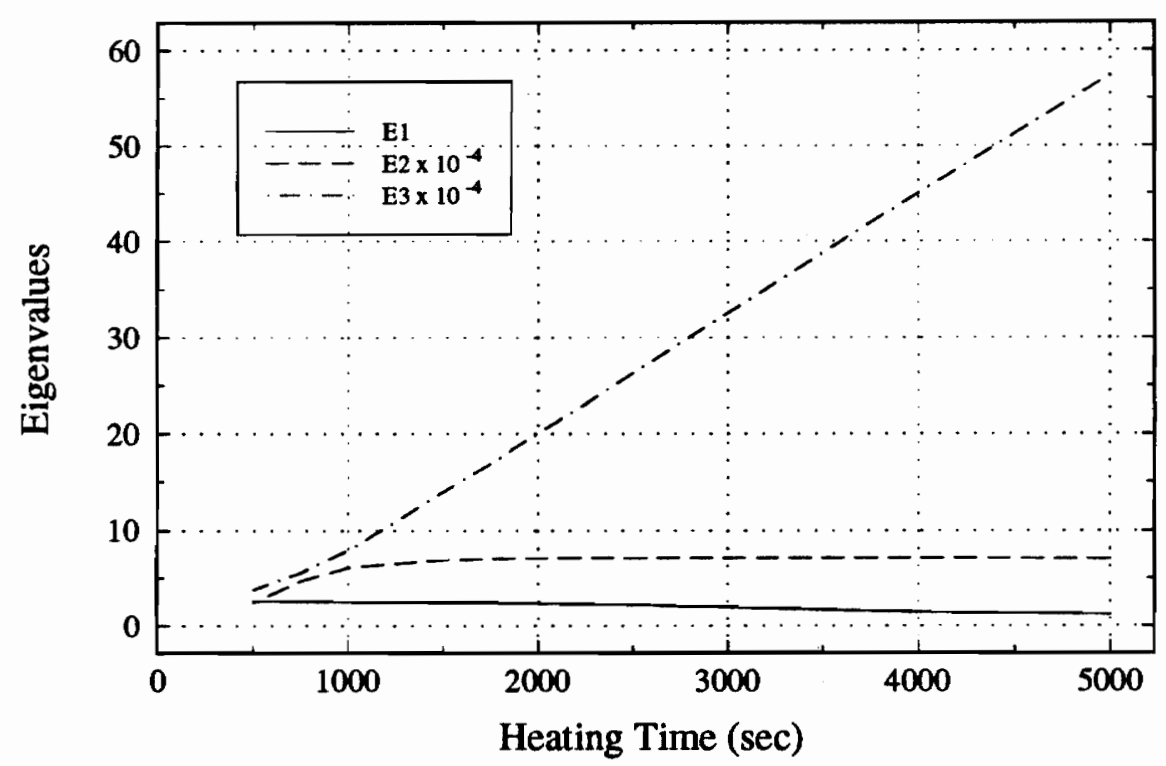

Figure A.5 Determinant Eigenvalues at $395 \mathrm{~K}$ for the Specified Temperature Boundary Case, Used to Determine Heating Time

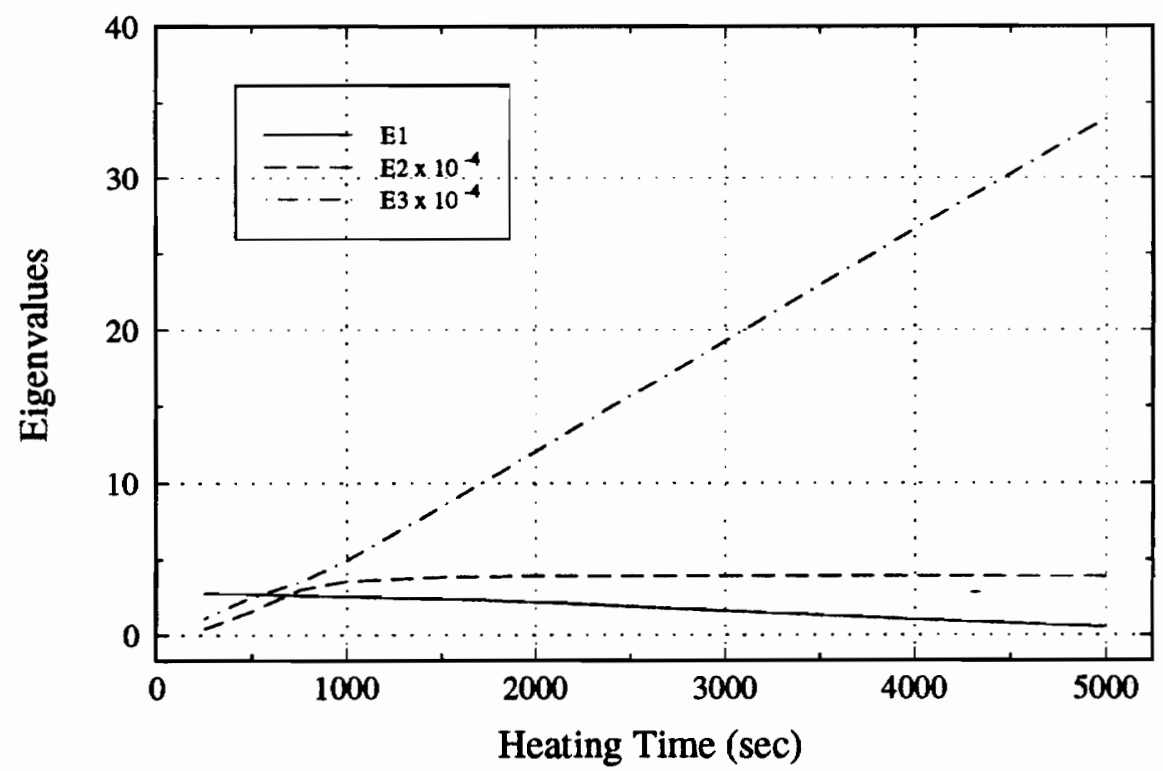

Figure A.6 Determinant Eigenvalues at $445 \mathrm{~K}$ for the Specified Temperature Boundary Case, Used to Determine Heating Time 


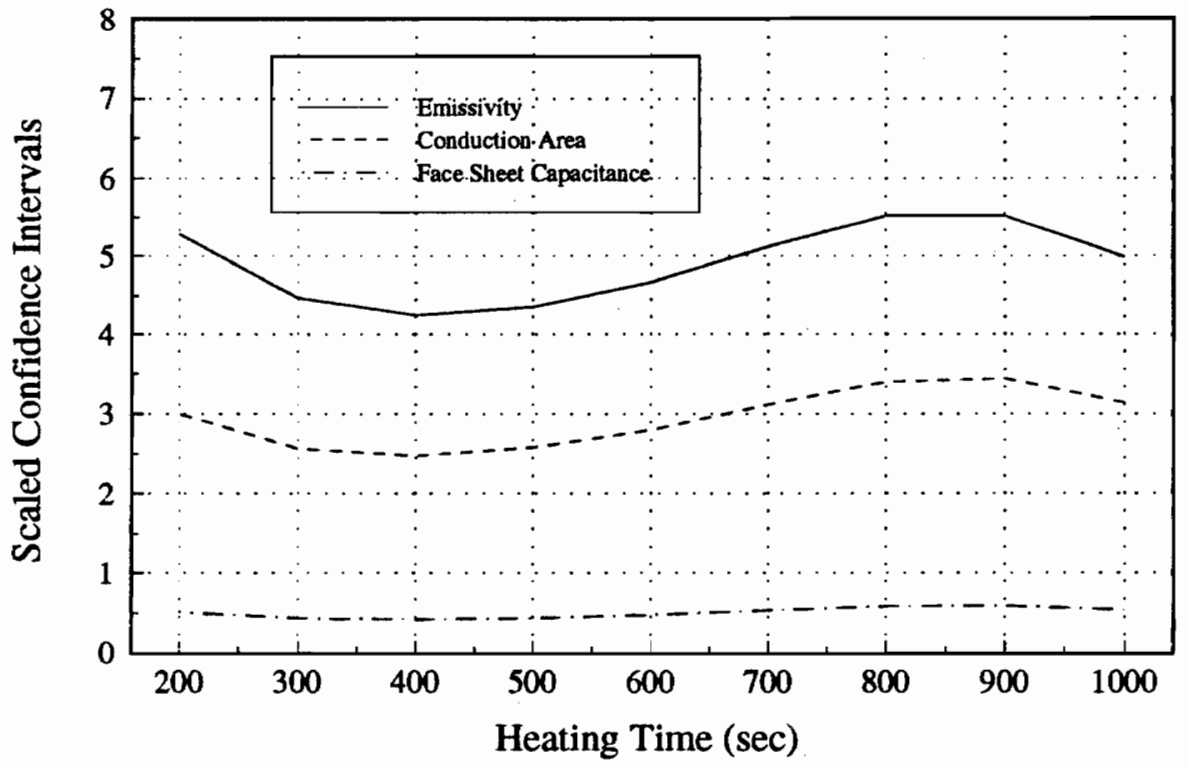

Figure A.7 Scaled Confidence Intervals at $345 \mathrm{~K}$ for the Insulated Boundary Case, Used to Determine Heating Time

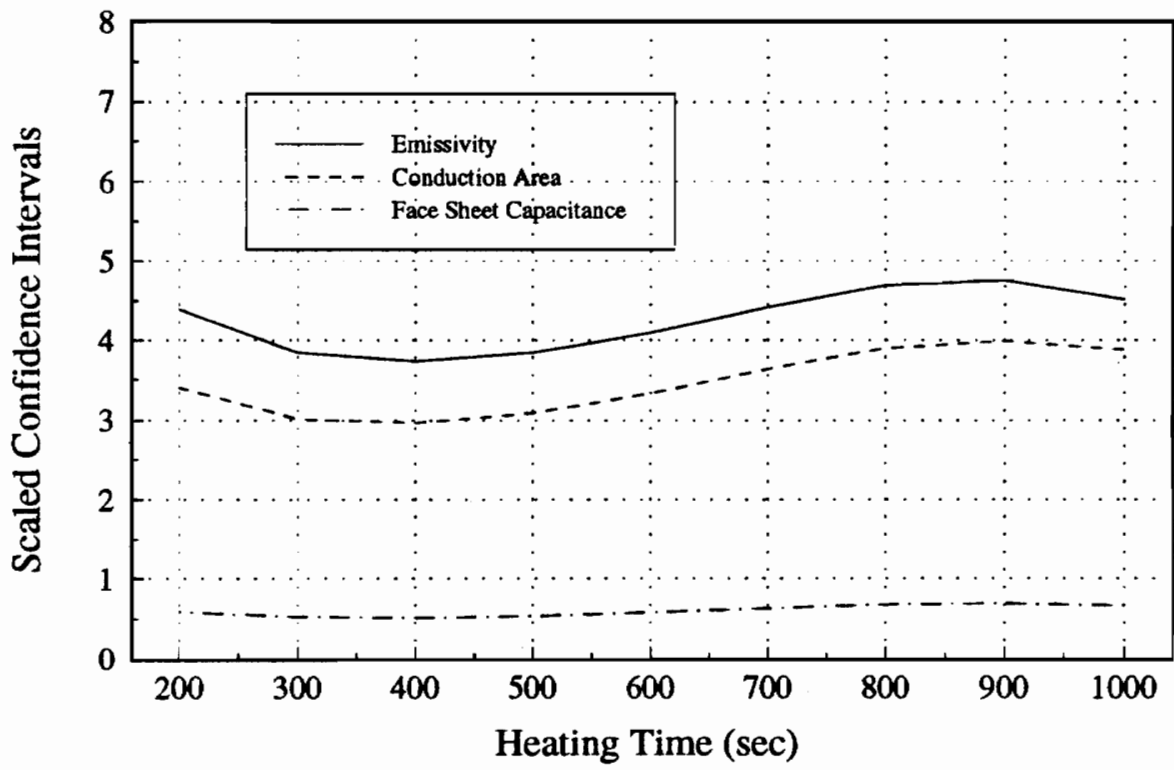

Figure A.8 Scaled Confidence Intervals at $395 \mathrm{~K}$ for the Insulated Boundary Case, Used to Determine Heating Time 


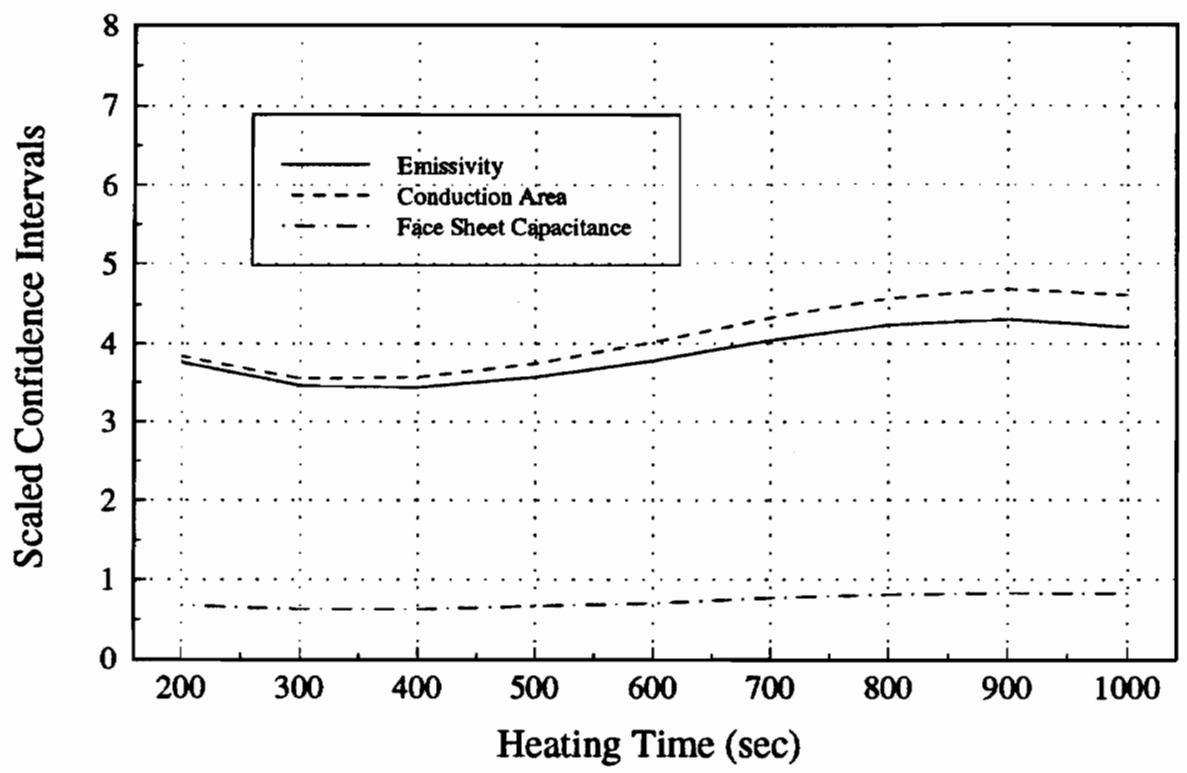

Figure A.9 Scaled Confidence Intervals at $445 \mathrm{~K}$ for the Insulated Boundary Case, Used to Determine Heating Time

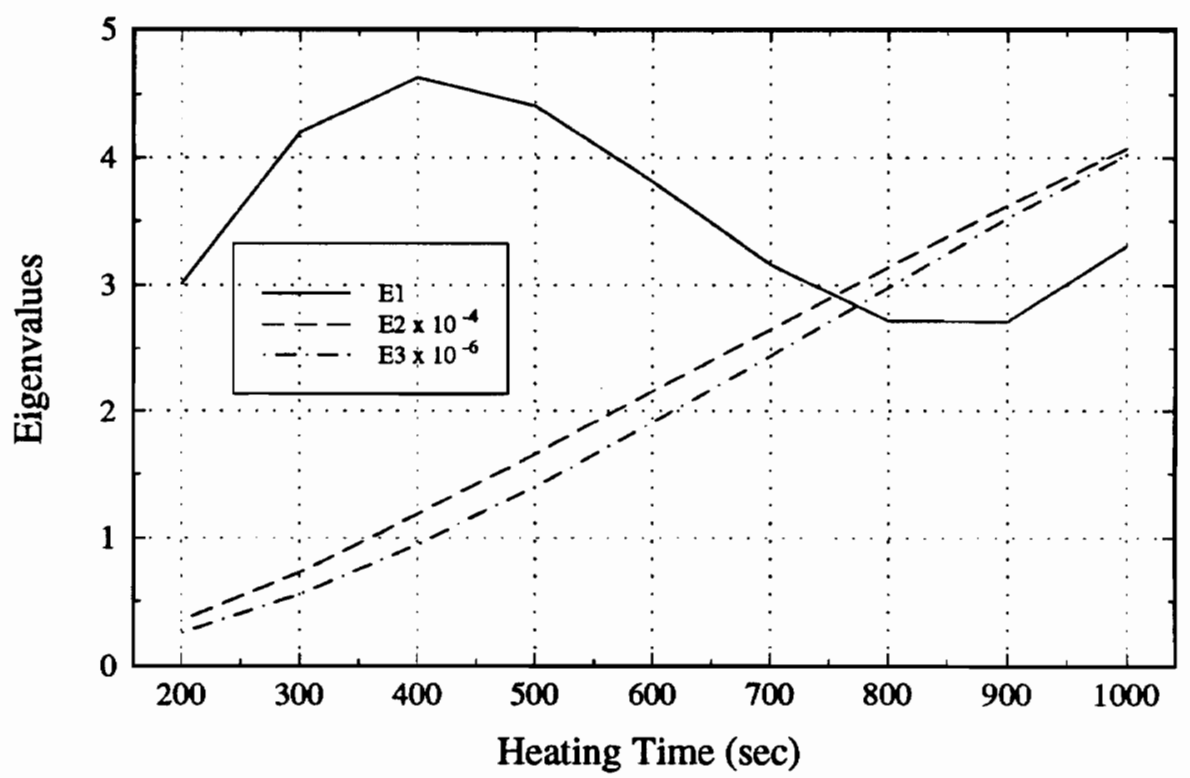

Figure A.10 Determinant Eigenvalues at $345 \mathrm{~K}$ for the Insulated Boundary Case, Used to Determine Heating Time 


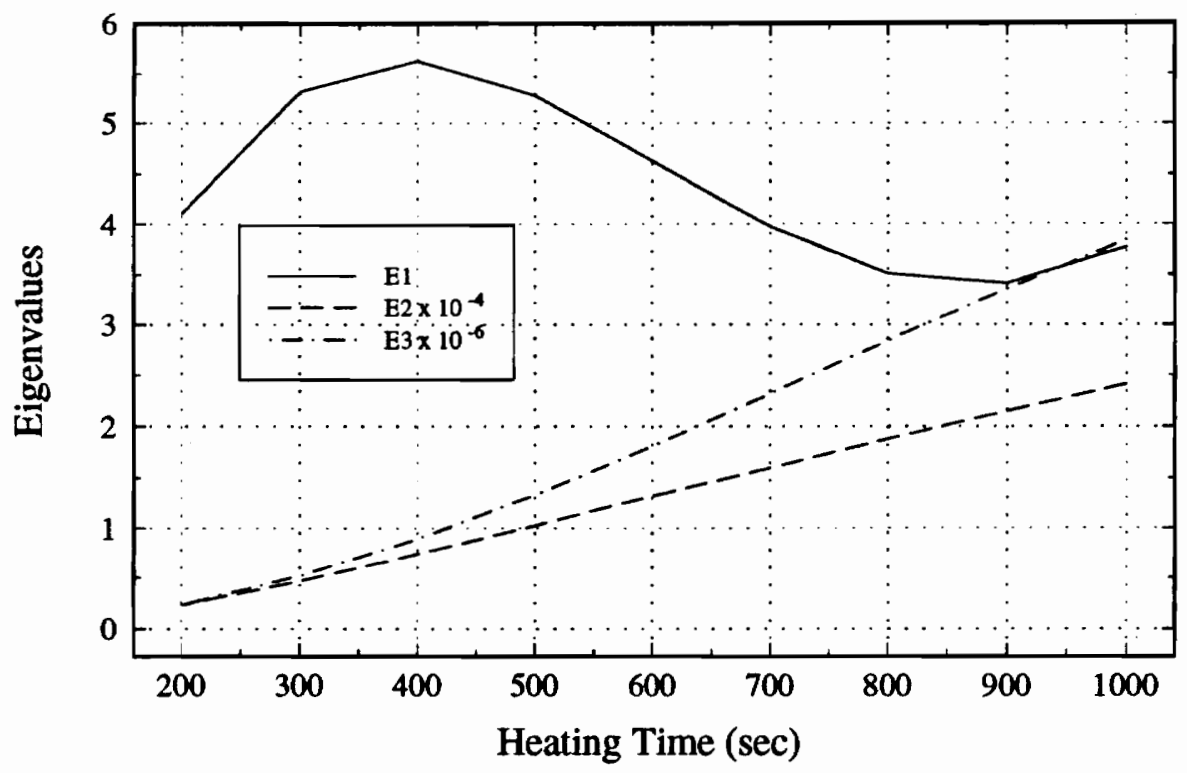

Figure A.11 Determinant Eigenvalues at $395 \mathrm{~K}$ for the Insulated Boundary Case, Used to Determine Heating Time

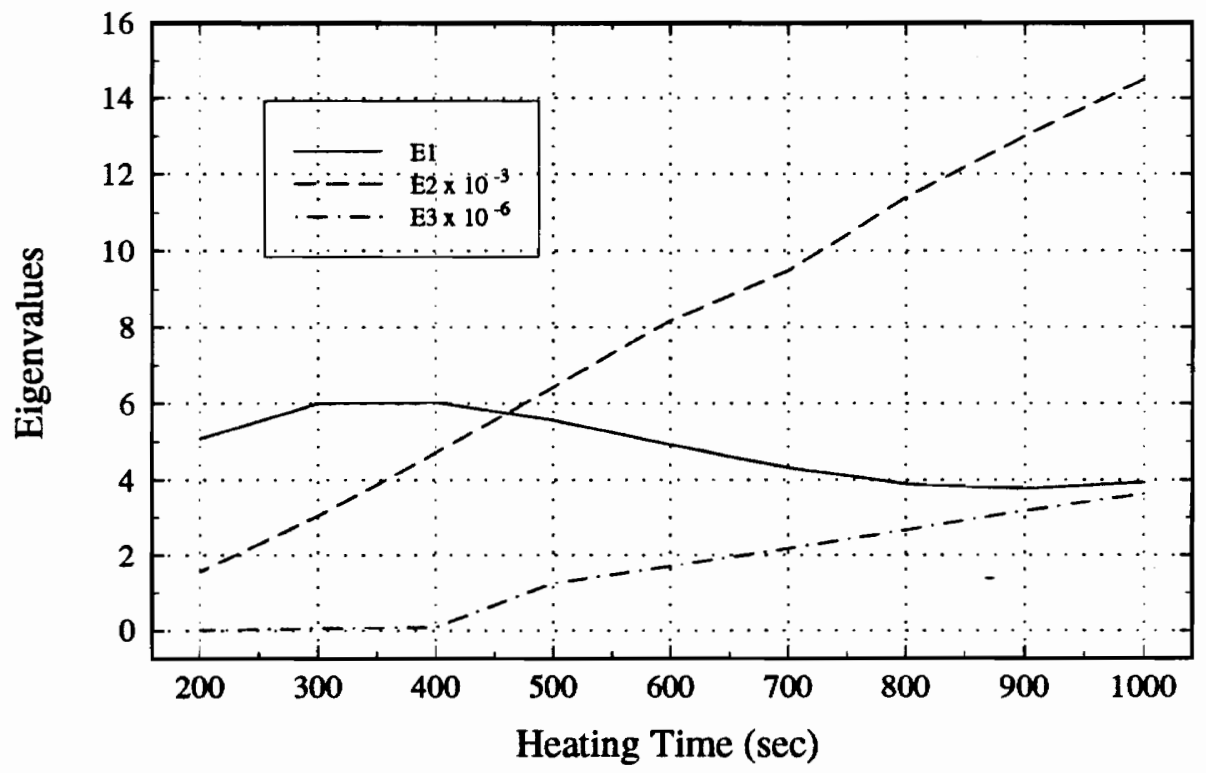

Figure A.12 Determinant Eigenvalues at $445 \mathrm{~K}$ for the Insulated Boundary Case, Used to Determine Heating Time 


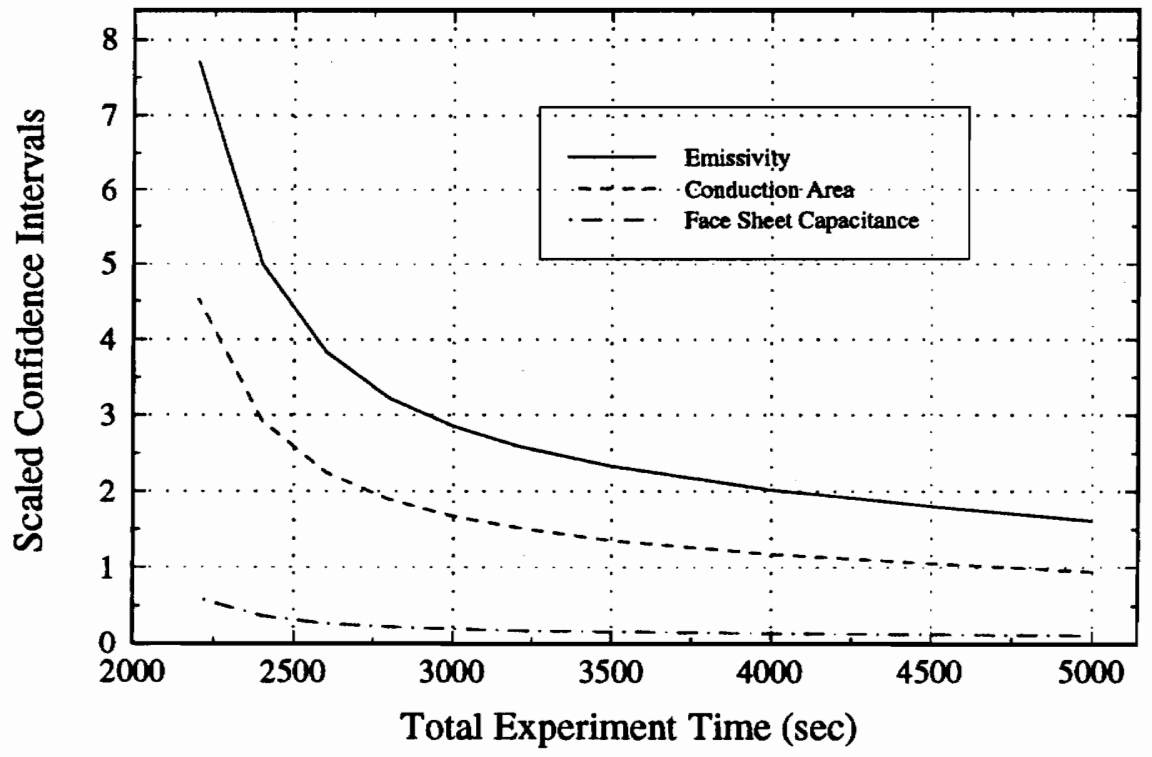

Figure A.13 Scaled Confidence Intervals at $345 \mathrm{~K}$ for the Specified Temperature Boundary Case Used to Determine Total Experiment Time

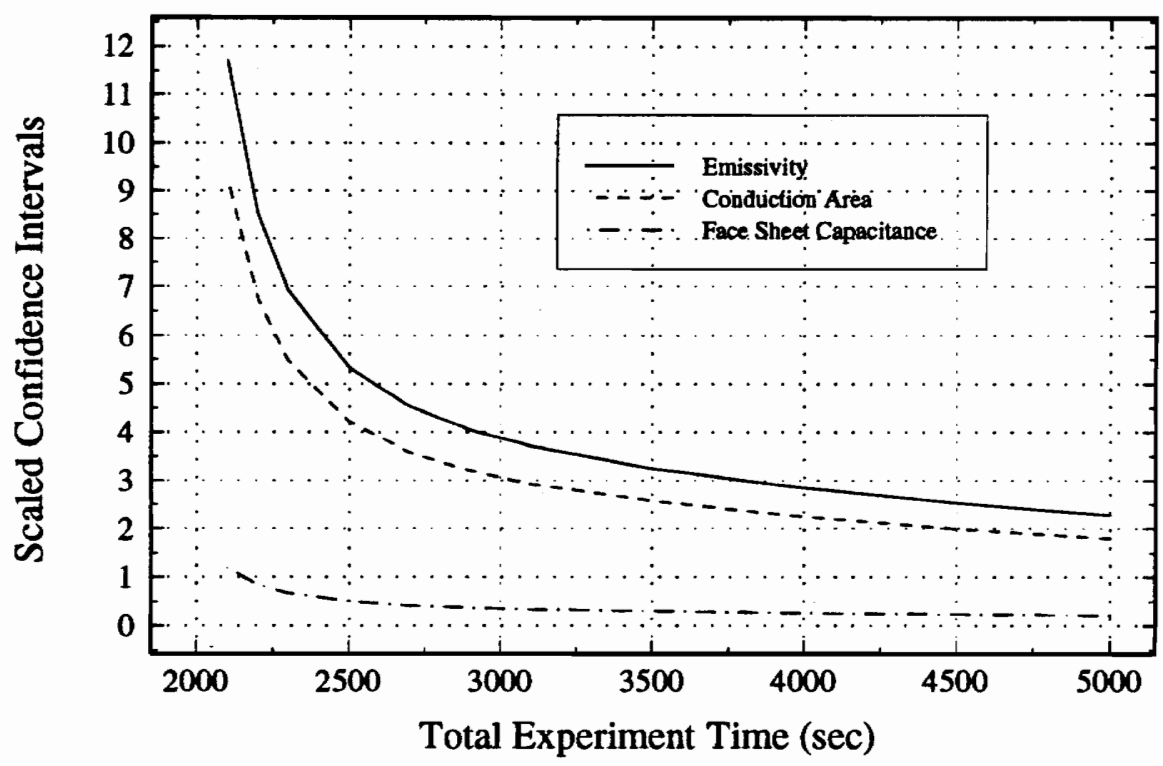

Figure A.14 Scaled Confidence Intervals at $395 \mathrm{~K}$ for the Specified Temperature Boundary Case, Used to Determine Total Experiment Time 


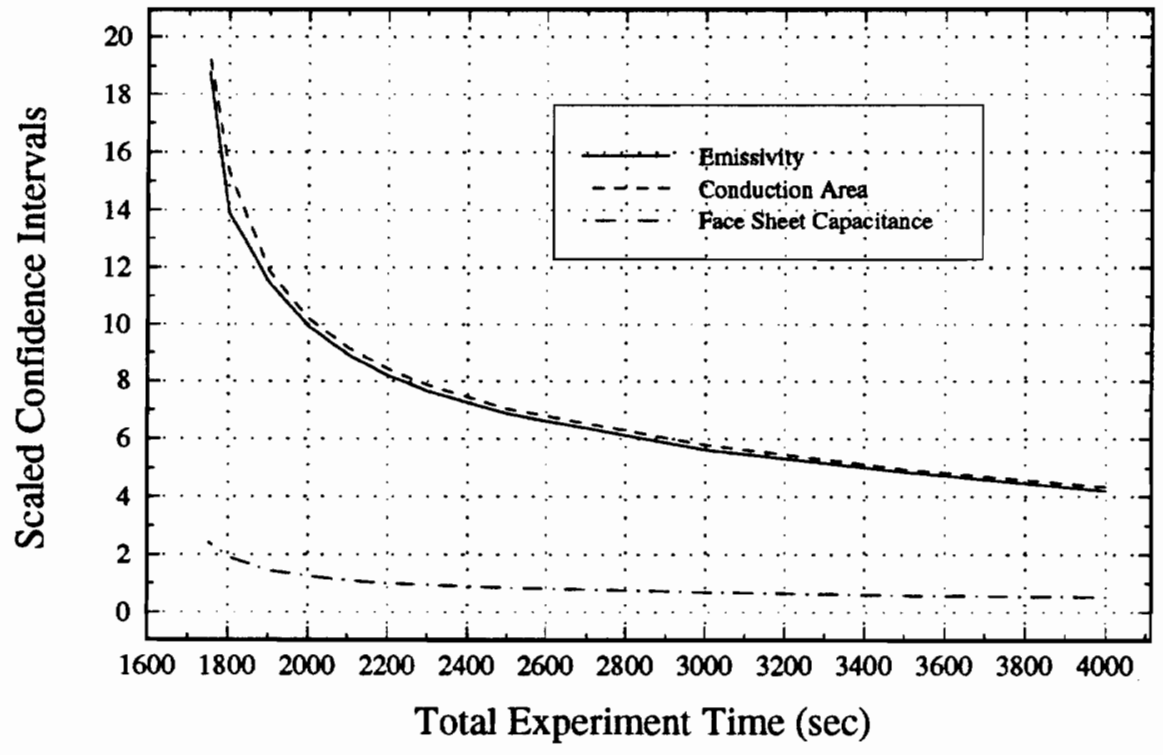

Figure A.15 Scaled Confidence Intervals at $445 \mathrm{~K}$ for the Specified Temperature Boundary Case, Used to Determine Total Experiment Time

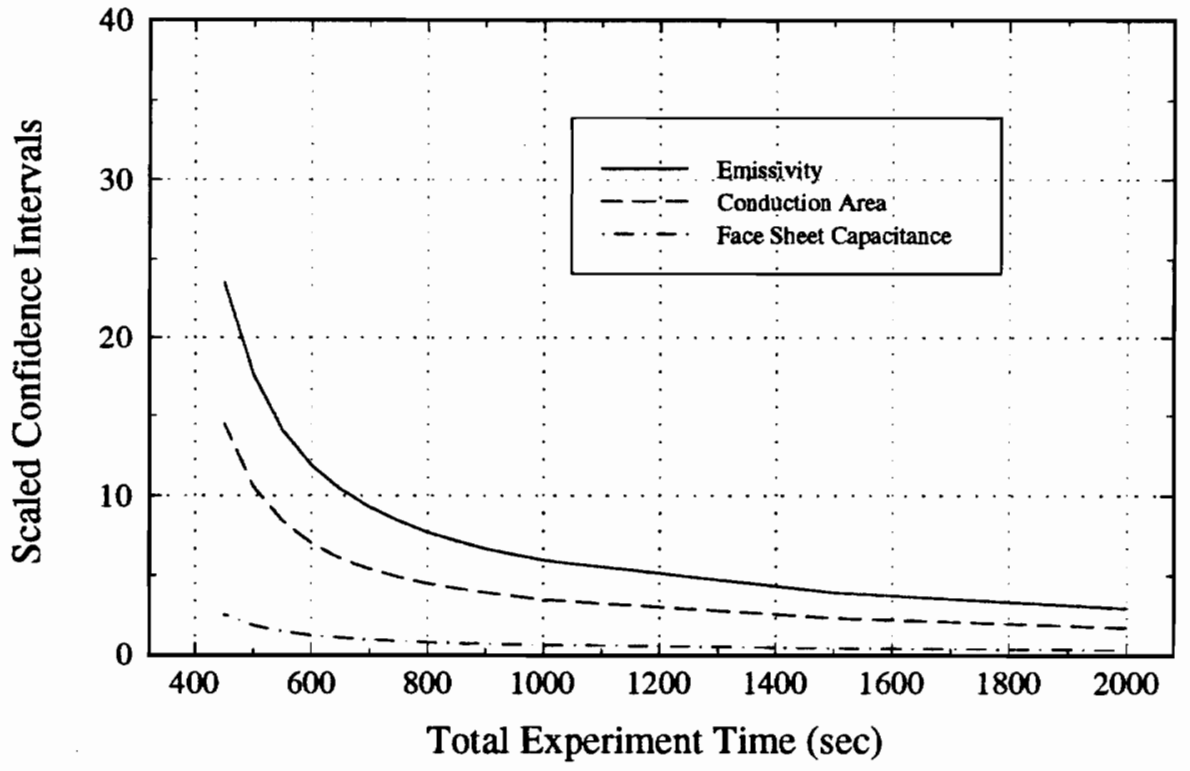

Figure A.16 Scaled Confidence Intervals at $345 \mathrm{~K}$ Used to Determine Total Experiment Time for the Insulated Boundary Case 


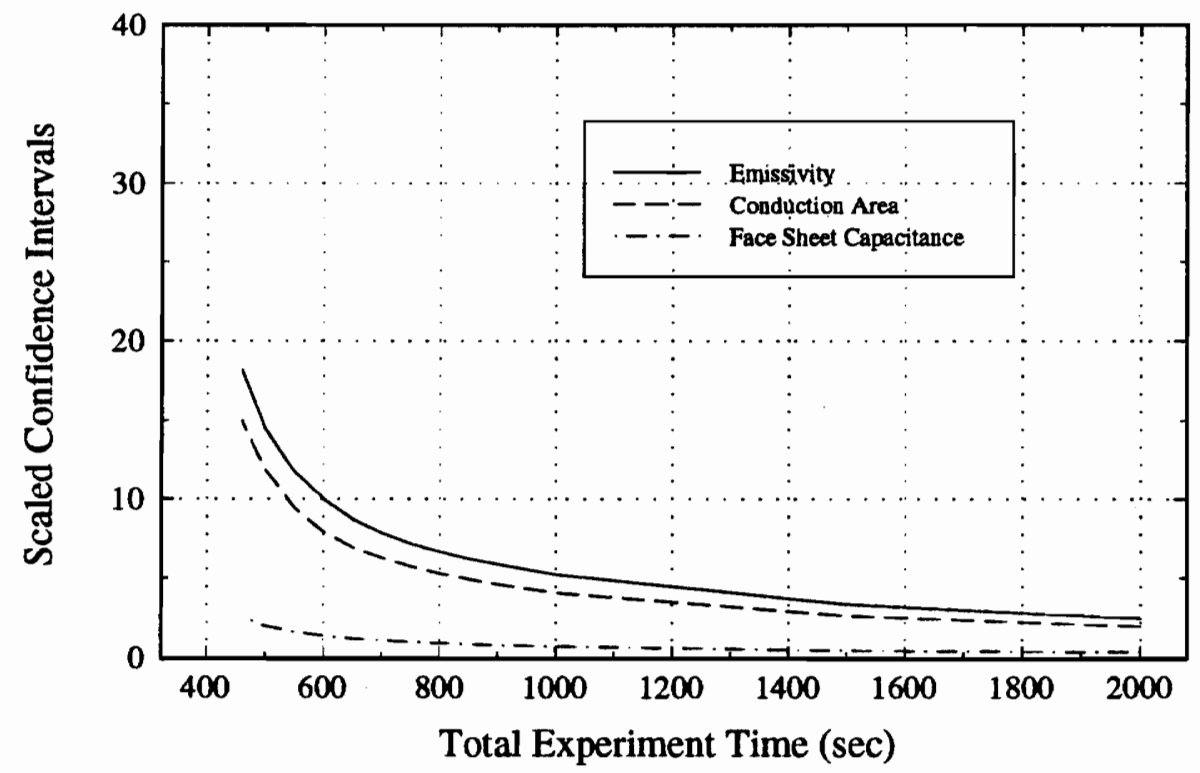

Figure A.17 Scaled Confidence Intervals at $395 \mathrm{~K}$ Used to Determine Total Experiment Time for the Insulated Boundary Case

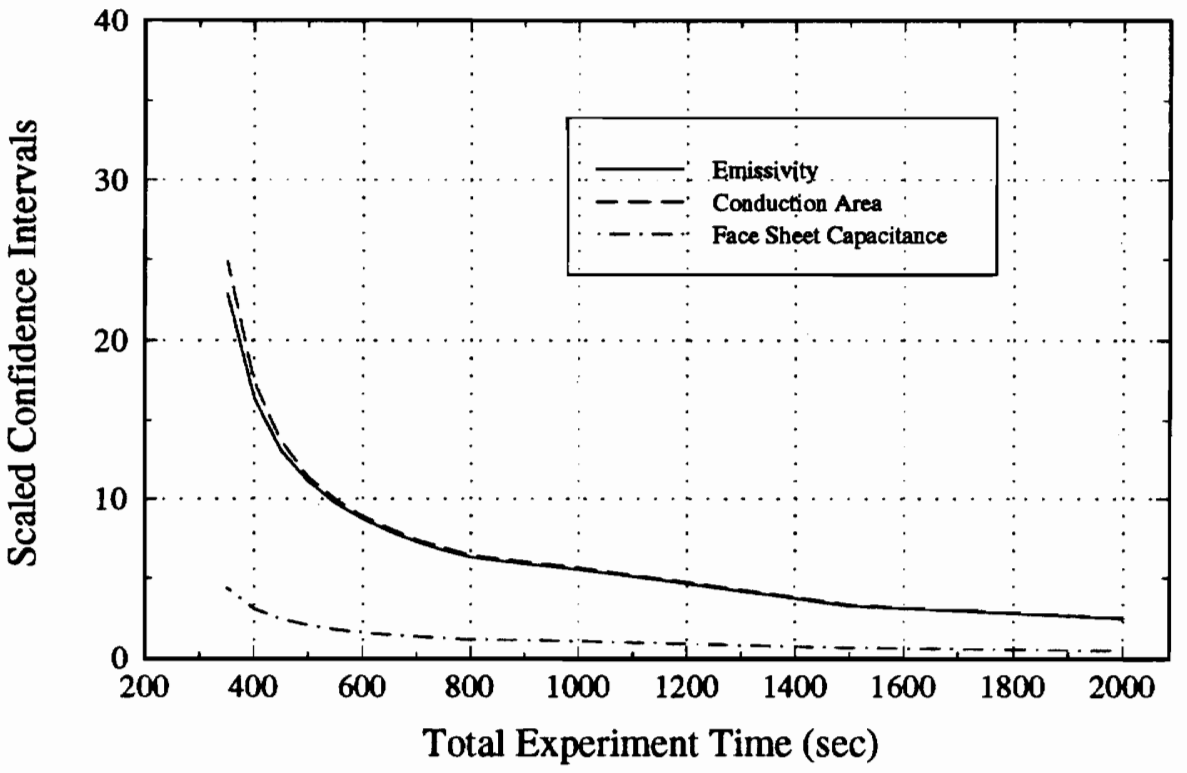

Figure A.18 Scaled Confidence Intervals at $445 \mathrm{~K}$ Used to Determine Total Experiment Time for the Insulated Boundary Case 


\section{APPENDIX B}

\section{Parameter Estimation Program: HCEST}

This appendix lists the EAL runstream used perform the parameter estimation. It is composed of three parts. The main part of the program forms the objective function and

calls the optimization processor, which minimizes it. Another part of the program HCSETUP is called once to initialize variables, read in data, and form the finite element model. The file titled HCTEMP is called at each iteration to calculate temperature histories. HCVF lists the view factors. The program shown is for estimation with both boundary conditions. If only one were used, then the case structure could be eliminated and only the components corresponding to the desired boundary condition would be kept.

Data files for the estimation procedure contain four columns, corresponding to time, temperature at the heated surface, temperature at the unheated surface, and heat flux. The columns of data need only be space delimited. The following header and format must appear: 
*XQT AUS

!NDS = (number of data points)

TABLE(NI=4,NJ="NDS"): INPT DATA: I = 1234

$\mathrm{J}=1$, "NDS"

$\mathrm{t} \quad \mathrm{T}_{\mathrm{hs}} \quad \mathrm{T}_{\text {uhs }} \quad \mathrm{q}^{\prime \prime}$

where $t=$ time

$\mathrm{T}_{\mathrm{hs}}=$ Temperature at the heated surface

$\mathrm{T}_{\text {uhs }}=$ Temperature at the unheated surface

$\mathrm{q}^{\mathrm{u}}$ = heat flux

The view factor file, HCVF, can have any number rows or columns. It must consistently list the emitting element number, incident element number, and view factor. 
\$PROGRAM HCEST - This program performs thermal property $\$$ estimation in a honeycomb core sandwich structure by $\$$ estimating emissivity, conduction area, and face sheet $\$$ capacitance. This is the control program.

$\$$

${ }^{*}$ XQT U1

*CMPARA(MAXCM=400000)

*(29 INIT ADS) ENDI

$\$$ USE INTERNAL DERIVES

*XQT AUS

TABLE(NI=5,NJ=1,TYPE=0): STAT ADS: J=1

01330 \$ exterior penalty $\mathrm{fn}$

TABLE(NI=1,N]=3): $X \quad$ ADS: J=1,3

$.70: 1 .: 1$. \$initial estimates

TABLE(NI=2,NJ=3): XB ADS: J=1,3: 0.4 1.: $0.110 .: 0.110$.

$\$$ TRUE $X 1=E M I S S$, OTHERS SCALED : this is the constraint vector !NDS2 $=2^{*}$ NDS $:$ !NDS4 $=2^{*}$ NDS2

TABLE(NI=1,NJ=2,TYPE=0): GTC ADS: INIT=ZERO

TABLE(NI=1,NJ=1): $\quad F$ ADS

TABLE(NI=1,NJ=2): G ADS

TABLE(NI=1,NJ="TOTL"): $\quad$ GT ADS

${ }^{*}$ XQT DCU

!ICT $=0$

*RETURN

*ENDI

$\$$

$\$$ The following subroutines change variables necessary for

$\$$ each data set

$\$$

*(29 FRST CASE) FRST

*XQT AUS

!NDS1 $=\mathrm{N} 1+1$

!NDS $=\mathrm{N} 1$

DEFINE $\mathrm{Q}=1$ SOUR TIME 21

DEFINE $R=1$ SOUR K21 22

DEFINE $S=1$ APPL TEMP 21

DEFINE $\mathrm{T}=1$ TEMP TIME 21

TABLE(NI=1,NJ="NDS1"): 1 SOUR TIME 11

TRANSFER(SOURCE=Q,SBASE $=0, I L I M=1, J L I M=" N D S 1 ", O P E R=X S U M$ )

TABLE(NI=1,N]="NDS1"):1 SOUR K21 21

TRANSFER(SOURCE=R,SBASE=0,ILIM=1,JLIM="NDS1",OPER=XSUM)

TABLE(NI=1,NJ="NDS"):1 APPL TEMP

TRANSFER(SOURCE=S,SBASE=0,ILIM=1,JLIM="NDS",OPER=XSUM)

TABLE(NI=1,NJ="NDS"):1 TEMP TIME 11

TRANSFER(SOURCE=T,SBASE=0,ILIM=1,JLIM="NDS",OPER=XSUM)

*XQT DCU

TOCC (1 SOUR K21 2 1): NJ=1,NINJ=1

TOCC (1 APPL TEMP ): NJ=1,NINJ=1

*RETURN 


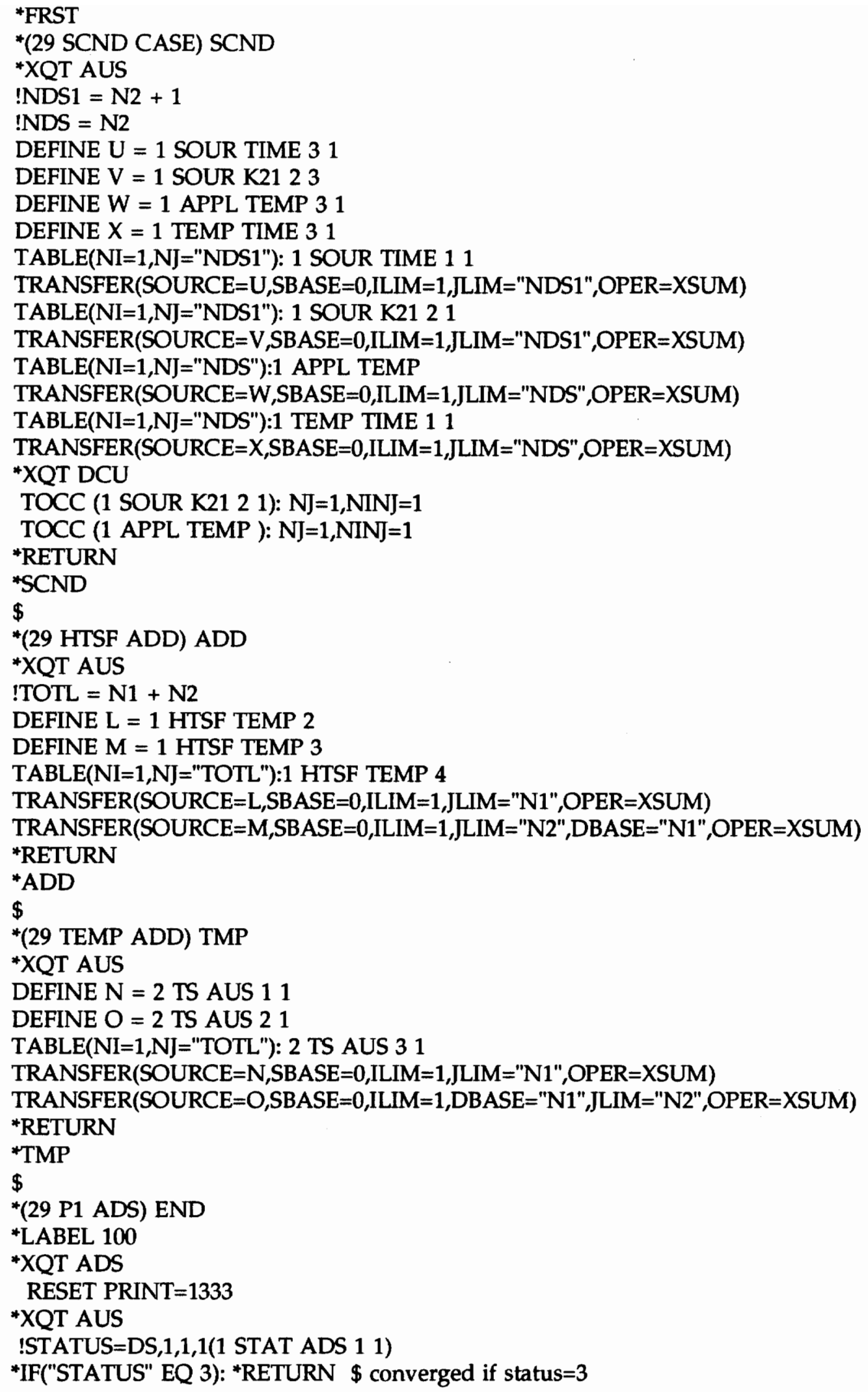




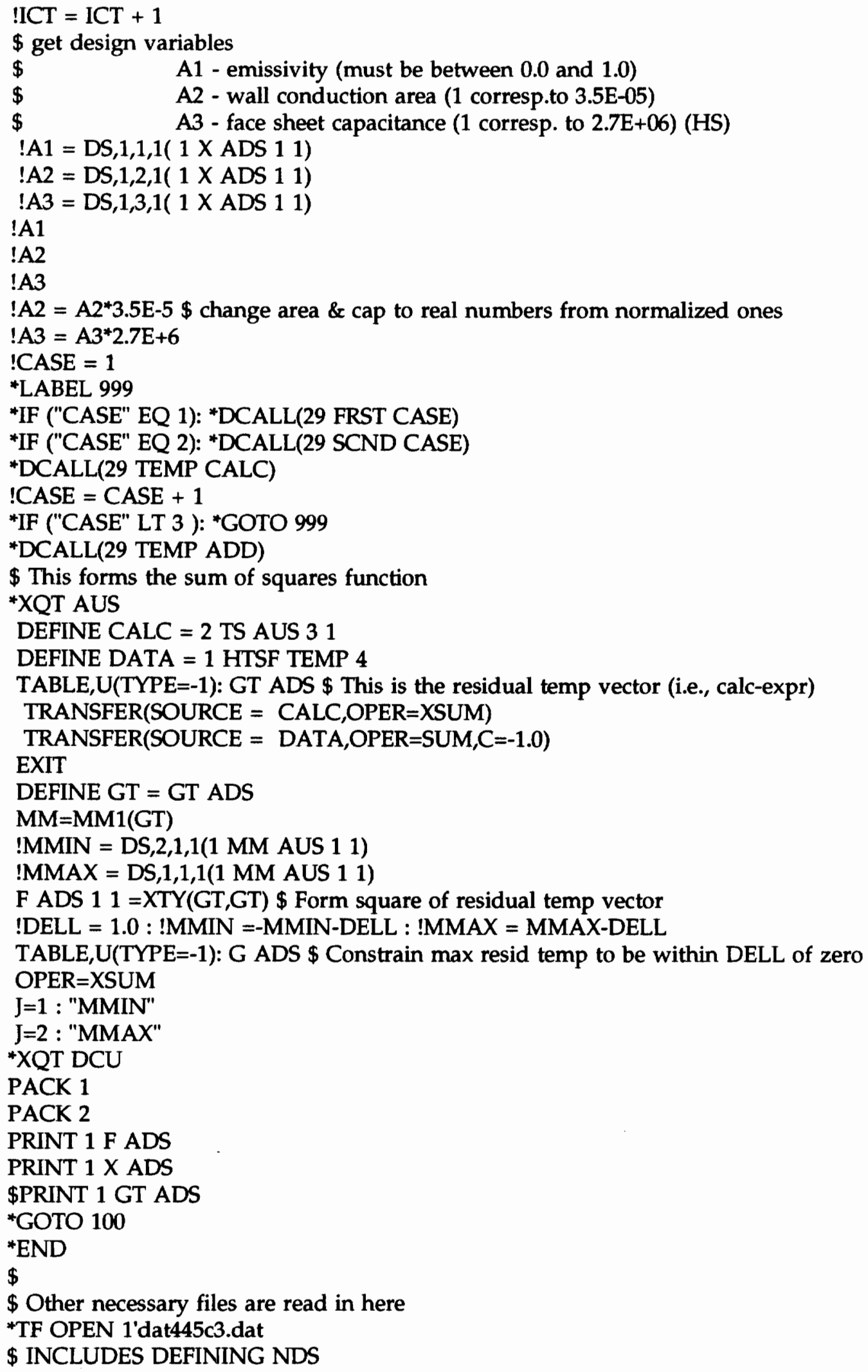


*TF READ 1

*TF CLOSE 1

*TF OPEN 1'dat445i3.dat

*TF READ 1

*TF CLOSE 1

*TF OPEN 1'hcvf

*TF READ 1

*TF CLOSE 1

*TF OPEN 1'hcsetupc

*TF READ 1

*XQT AUS

*TF CLOSE 1

*TF OPEN 1'hctempc

*TF READ 1

*TF CLOSE 1

*DCALL (29 INPT VARB)

*DCALL(29 PROG INIT)

*DCALL(29 HTSF ADD)

*DCALL(29 INIT ADS)

*DCALL(29 P1 ADS)

*XQT EXIT 


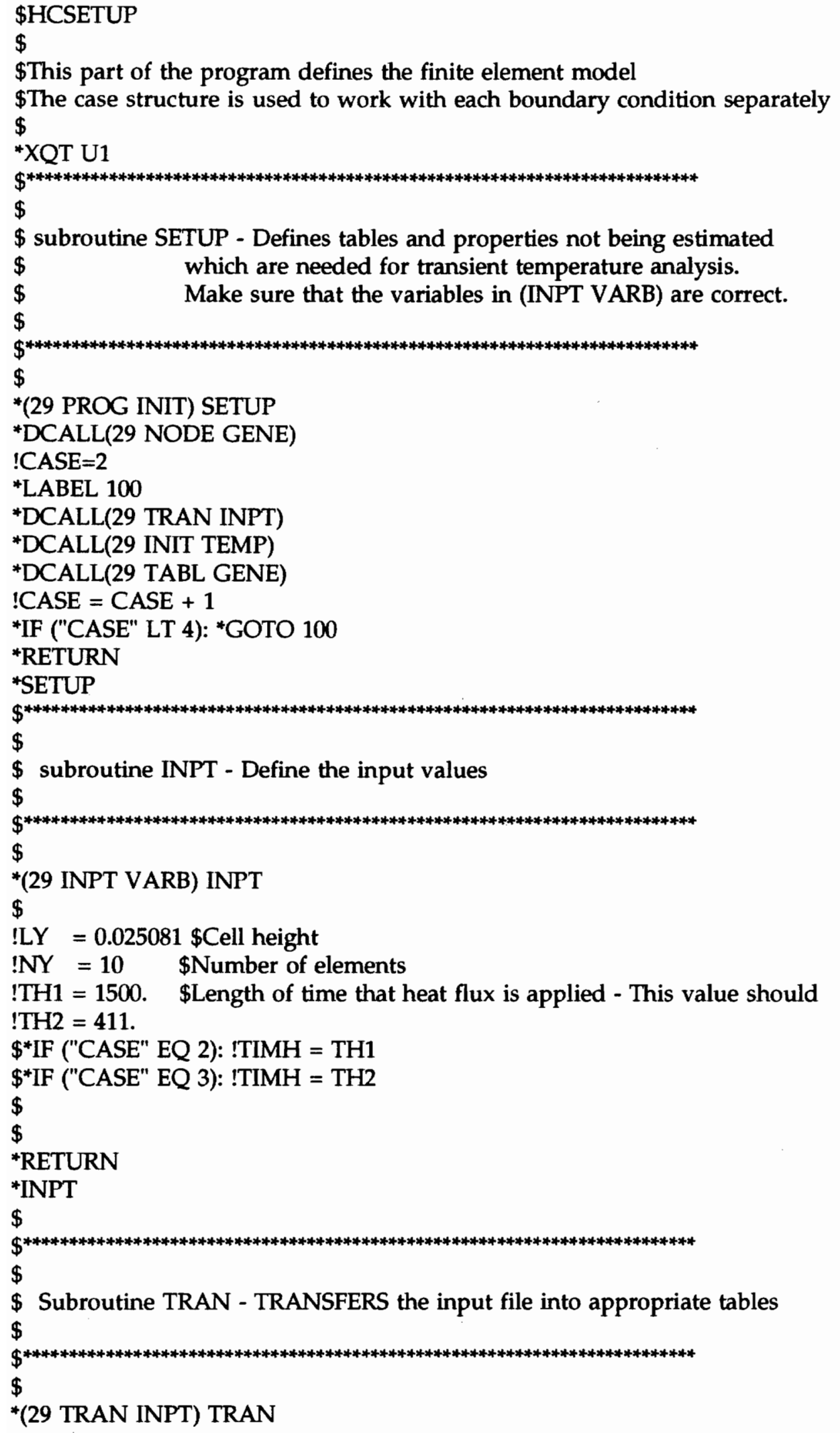




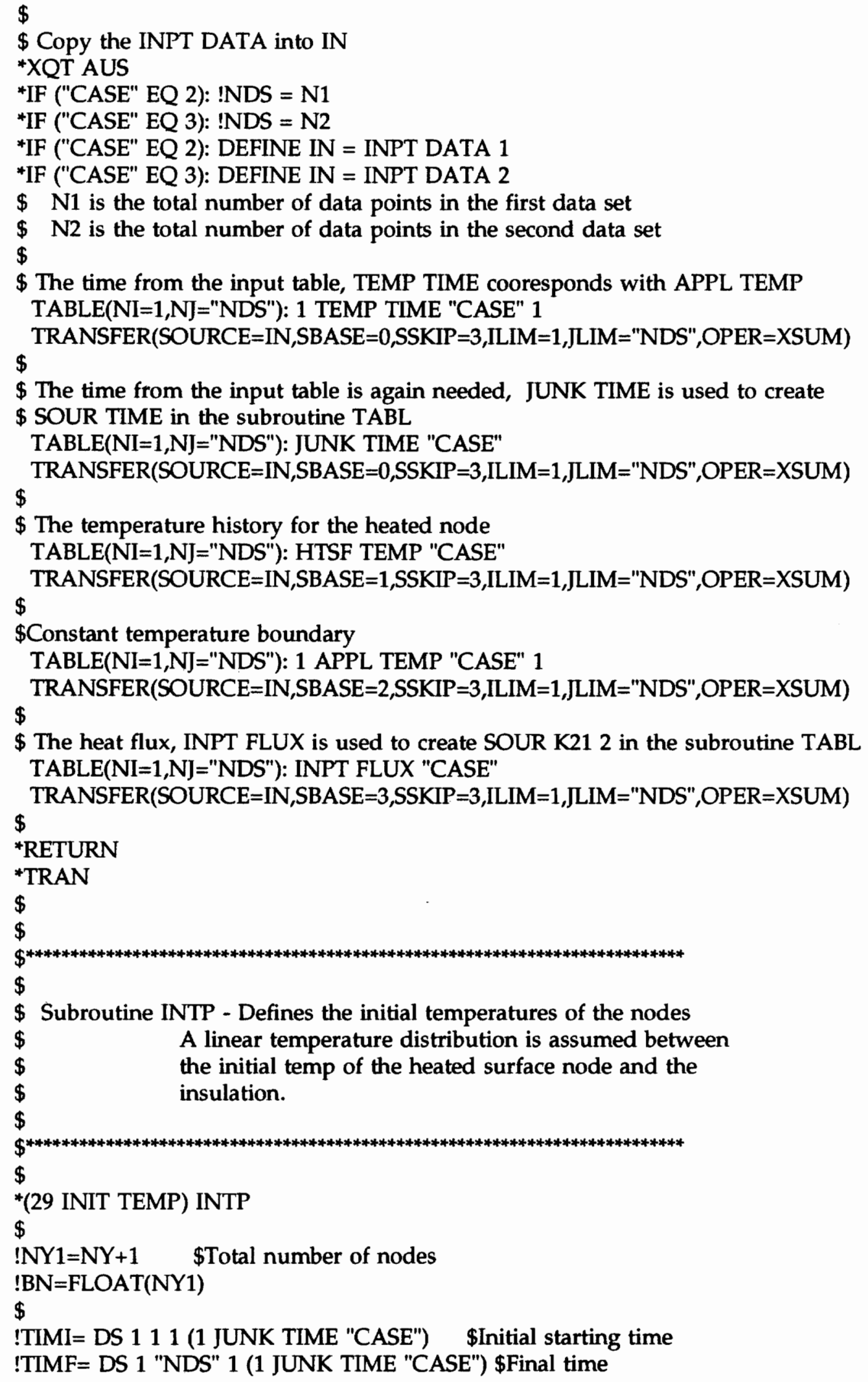




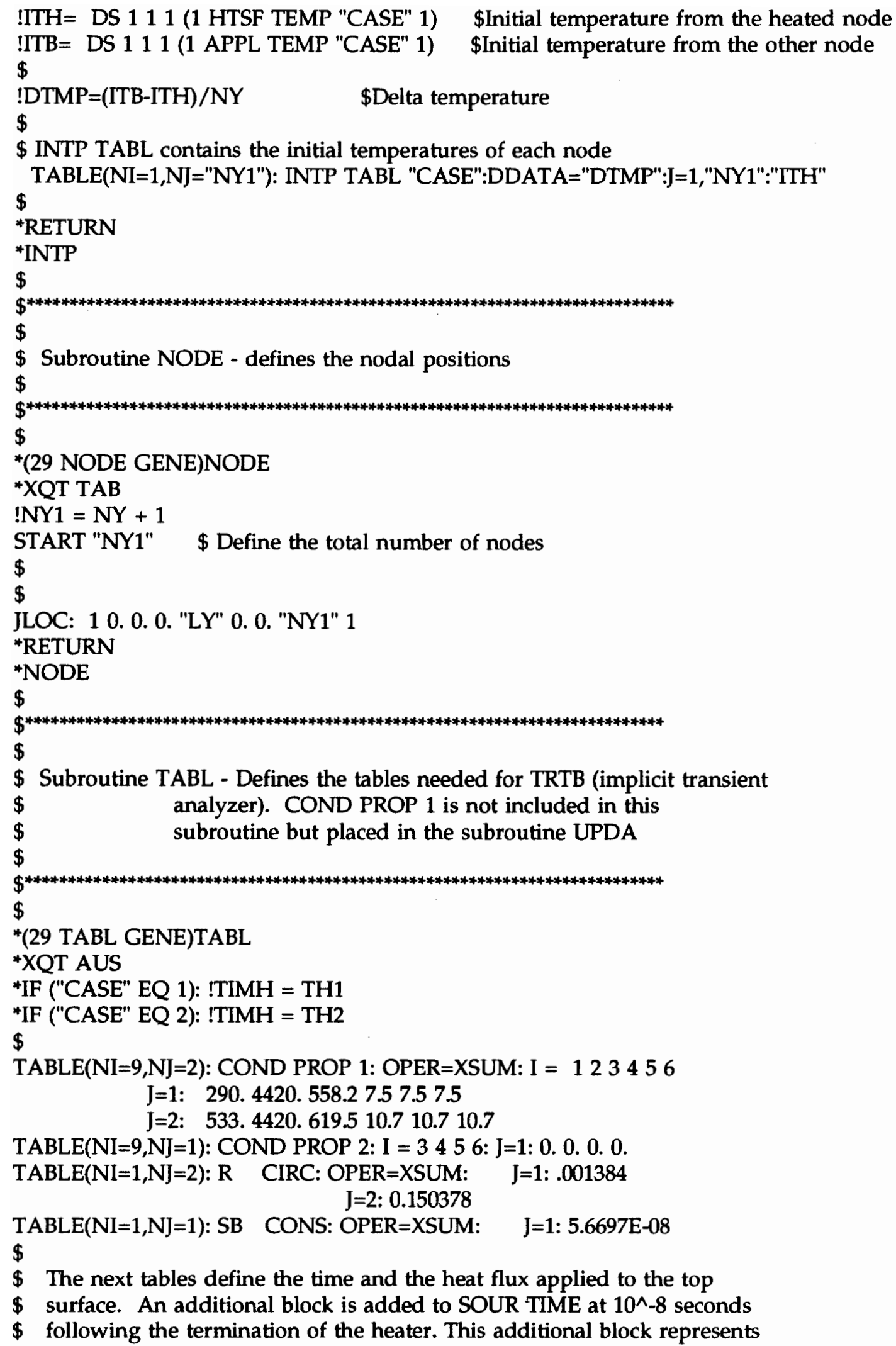


$\$$ the time at which the heater is turned off. To coorespond with this

$\$$ additional block in SOUR TIME an additional block needs to be added

\$ to SOUR K21 2. The value used for SOUR K21 2 is the duplicate of the

$\$$ heat flux immediately following the termination of the heater.

$\$$

$\$$ First determine where the additional block needs to be added. Once the

$\$$ time is greater than or equal to the heater time, TIMH, the block should

$\$$ be added.

$\$$

!CTR=0

*LABEL 987

!CTR $=$ CTR + 1

!TYME=DS 1 "CTR" 1 ( 1 JUNK TIME "CASE")

*IF( "TYME" LE "TIMH"):*GOTO 987

!BLKN=CTR

!CTR=BLKN-1

!END=NDS-CTR

$\$$

!SMAL $=1 . E-8 \quad$ \$value added to TIMH to define when the heat flux turns

!THDL $=$ TIMH + SMAL \$off, this is needed due to the discontinuity at TIMH

$\$$

!BN=FLOAT(NY1)

TABLE(NI=1,NJ=1): TEMP NODE: OPER=XSUM:J=1:"BN"

$\$$ Create a table to hold the additional time step value: THDL $=\mathrm{TIMH}+\mathrm{SMAL}$

TABLE(NI=1,NJ=1): 1 THDL TBLE "CASE":J=1:"THDL"

DEFINE A = THDL TBLE "CASE"

$\$$

DEFINE B = JUNK TIME "CASE" \$The initial time history

$\$$

$\$$ Create the table: SOUR TIME to hold the initial time history plus the

$\$$ additional time step THDL

!NDS1 $=$ NDS +1

TABLE(NI=1,NJ="NDS1"): 1 SOUR TIME "CASE" 1

TRANSFER(SOURCE=B,SBASE=0,SSKIP=0,ILIM=1,JLIM="CTR",OPER=XSUM)

TRANSFER(SOURCE=A,SBASE=0,DBASE="CTR",SSKIP=0,ILIM=1,JLIM=1,OPER=XSUM)

TRANSFER(SOURCE=B,SBASE="CTR",DBASE="BLKN",SSKIP=0,ILIM=1,JLIM="END",OPER=X SUM)

$\$$

!FLX=DS 1 "BLKN" 1 (1 INPT FLUX) \$The duplicate flux following the heater turn

$\$$ off

$\$$

$\$$ Create the table to hold the additional FLX

TABLE(NI=1,NJ=1):1 FLUX TBLE:OPER=XSUM:J=1:"FLX"

DEFINE C $=$ FLUX TBLE

$\$$

DEFINE D = INPT FLUX \$Orginal heat flux history

$\$$

$\$$ Create SOUR K21 2 including the original flux history and the additional FLX

TABLE(NI=1,NJ="NDS1"): 1 SOUR K21 2 "CASE"

TRANSFER(SOURCE=D,SBASE=0,SSKIP=0,ILIM=1,JLIM="CTR",OPER=XSUM)

TRANSFER(SOURCE=C,SBASE=0,DBASE="CTR",SSKIP=0,ILIM=1,JLIM=1,OPER=XSUM) 
TRANSFER(SOURCE=D,SBASE="CTR",DBASE="BLKN",SSKIP=0,ILIM=1,JLIM="END",OPER= XSUM)

$\$$

$\$$

$\$ *$ XQT DCU

$\$$ TOCC (1 SOUR K21 2 "CASE"):NJ=1,NINJ=1

$\$$ TOCC (1 APPL TEMP "CASE"): NJ=1,NINJ=1

$\$$ SOUR K21 2 must be in the for of NDS $1 \times 1$ blocks

$\$$ PRINT 1 SOUR TIME

$\$ * X Q T$ AUS

$\$$ PRINT 1 SOUR TIME

$\$$

*RETURN

"TABL 


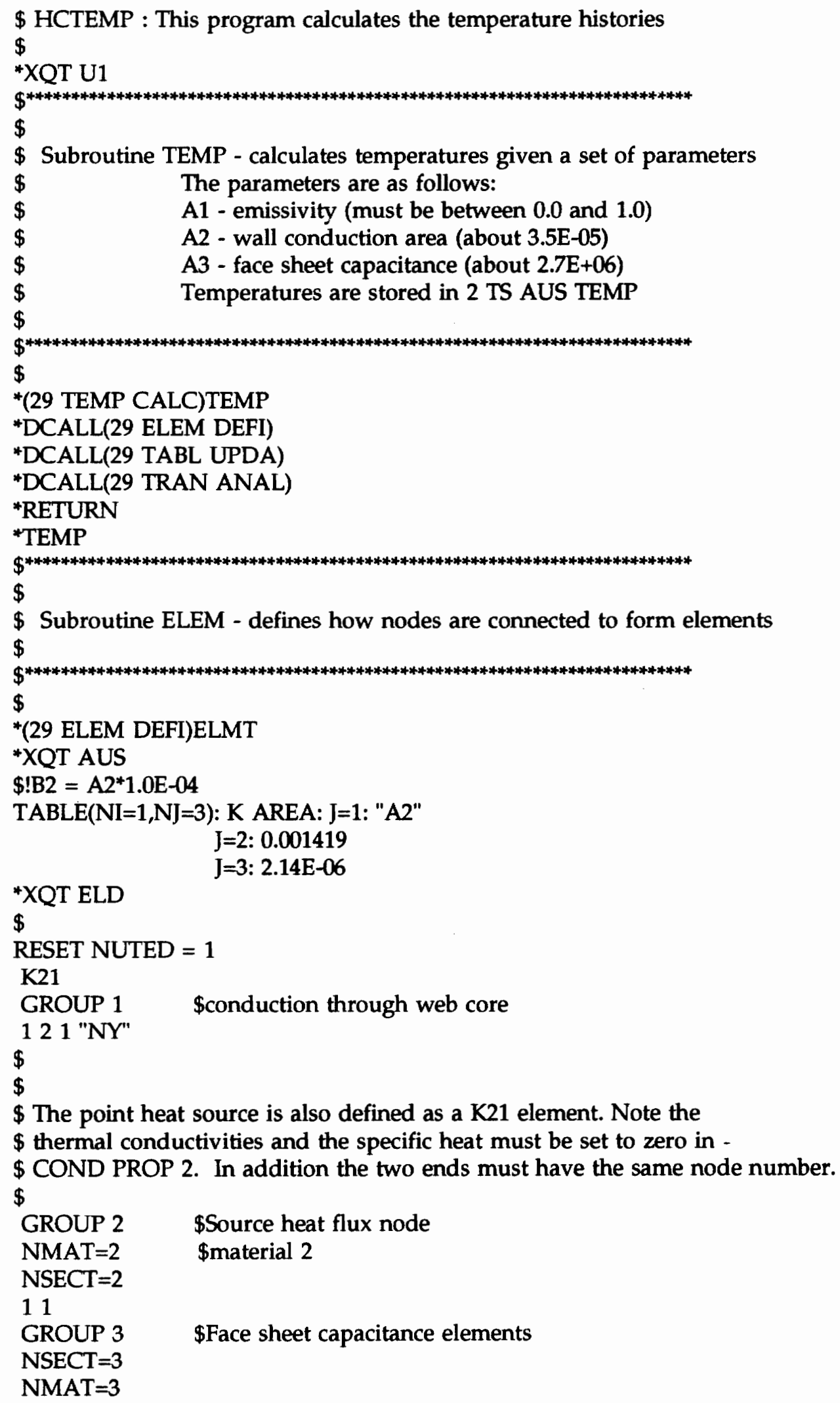




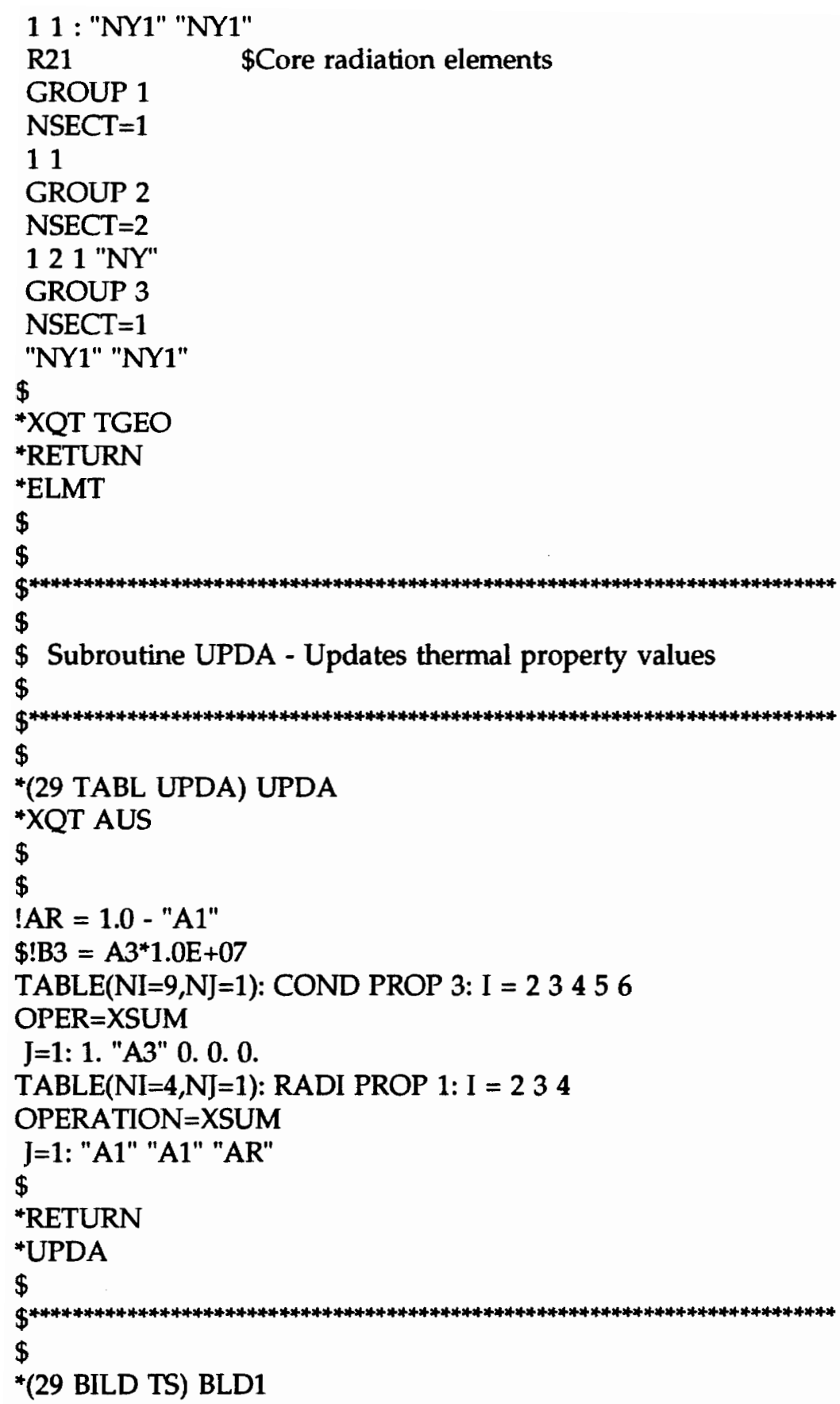




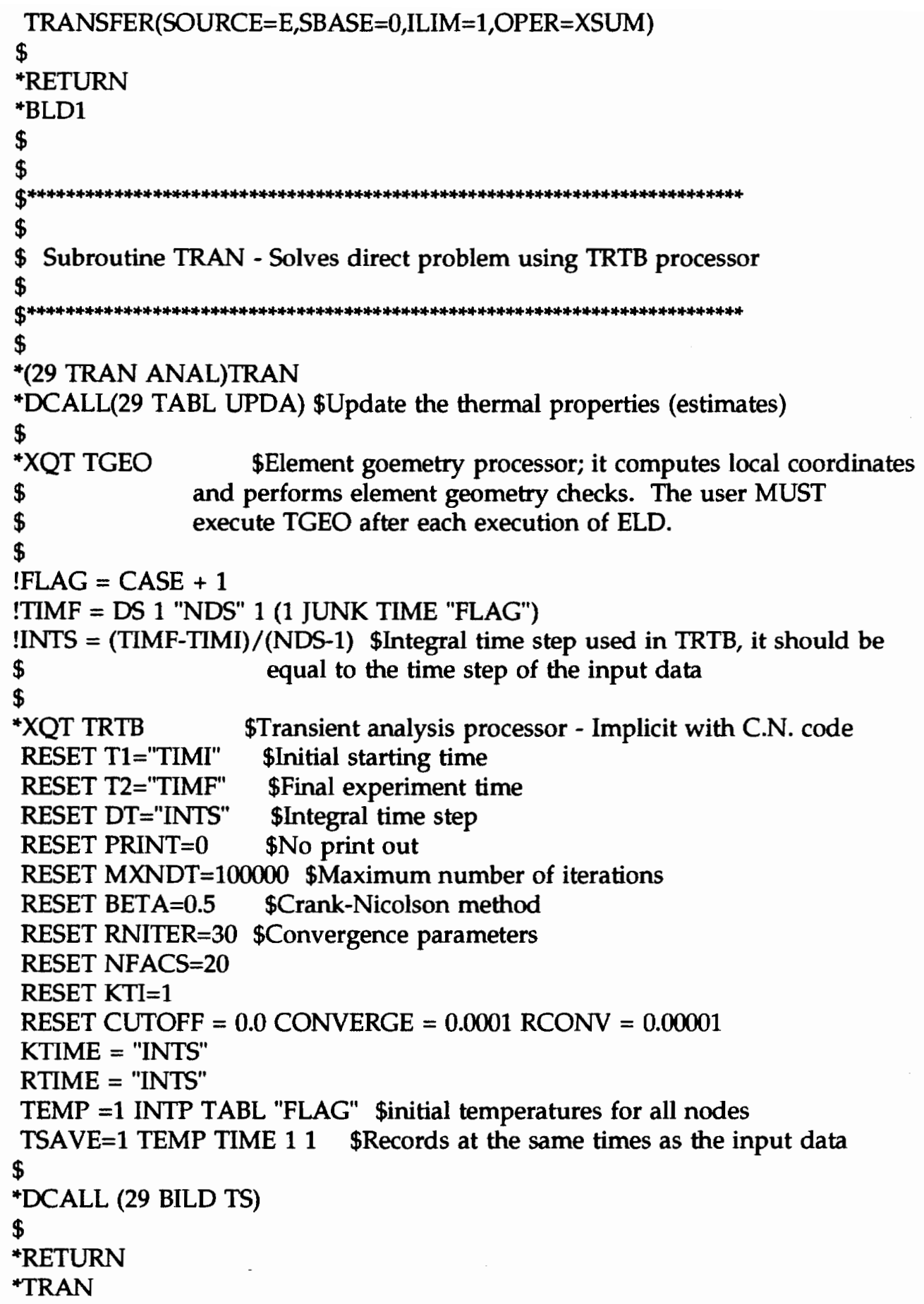


$\$$ This is the set of radiation view factors ${ }^{*}$ XQT AUS

TABLE(NI=13,NJ=24 ): REX VF: J=1,24

1. 1. 0. 2. 0.122619 3. 0.103627 4. 0.089085 5. 0.0770746 .0 .067029

1. 7. 0.058423 8. 0.0508359 .0 .044613 10. 0.03878811 .0 .03408412 .0 .313824

2. 1. 0.450108 2. 0.099344 3. 0.07002 4. 0.053073 5. 0.0440216 .0 .037191

2. 7. 0.031944 8. 0.0273619 .0 .02354210 .0 .0203911 .0 .01757312 .0 .125433

3. 1.0 .380325 2. 0.070165 3. 0.09938 4. 0.069741 5. 0.0530736 .0 .043882

3. 7. 0.037027 8. 0.0318859 .0 .02737510 .0 .02366311 .0 .02036212 .0 .143122

4. 1. 0.327392 2. 0.053125 3. 0.070081 4. 0.099375 5. 0.069926 .0 .053031

4. 7. 0.043802 8. 0.0373229 .0 .031809 10. 0.02747211 .0 .023597 12. 0.163074

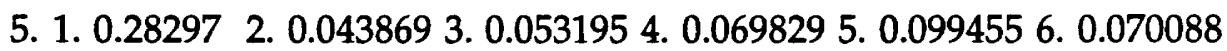

5. 7. 0.0533368 .0 .0438829 .0 .03708610 .0 .03178811 .0 .02767412 .0 .186826

6. 1. 0.246134 2. 0.037041 3. 0.043813 4. 0.0532185 .0 .06969 6. 0.099458

6. 7. 0.070029 8. 0.053235 9. 0.04370410 .0 .03699311 .0 .031834 12. 0.214853

7. 1. 0.214853 2. 0.031834 3. 0.036993 4. 0.043704 5. 0.0532356 .0 .070029

7. 7. 0.099458 8. 0.06969 9. 0.05321810 .0 .04381311 .0 .037041 12. 0.246134

8. 1. 0.186826 2. 0.027674 3. 0.031788 4. 0.0370865 .0 .043882 6. 0.053336

8. 7. 0.070088 8. 0.099455 9. 0.069829 10. 0.05319511 .0 .043869 12. 0.28297

9. 1. 0.163074 2. 0.023597 3. 0.027472 4. 0.031809 5. 0.037322 6. 0.043802

9. 7. 0.0530318 .0 .069929 .0 .09937510 .0 .070081 11. 0.05312512 .0 .327392

10. 1.0 .143122 2. 0.020362 3. 0.023663 4. 0.027375 5. 0.031885 6. 0.037027

10. 7. 0.0438828 .0 .053073 9. 0.069741 10. 0.09938011 .0 .07016512 .0 .380325

11. 1. 0.125433 2. 0.017573 3. 0.020390 4. 0.023542 5. 0.0273616 .0 .031944

11. 7. 0.037191 8. 0.0440219 .0 .05307310 .0 .0700211 .0 .09934412 .0 .450108

12. 1.0 .313824 2. 0.034084 3. 0.038788 4. 0.044613 5. 0.0508356 .0 .058423

12. 7. 0.067029 8. 0.0770749 .0 .08908510 .0 .10362711 .0 .12261912 .0 . 


\section{APPENDIX C}

\section{Optimization Program: OPT}

This program can be used to perform either optimization (experimental design) or to calculate confidence intervals for estimates. All that is necessary is to use the correct set of parameters and to use the correct sum of squares value.

\section{Table C.1 Program OPT}

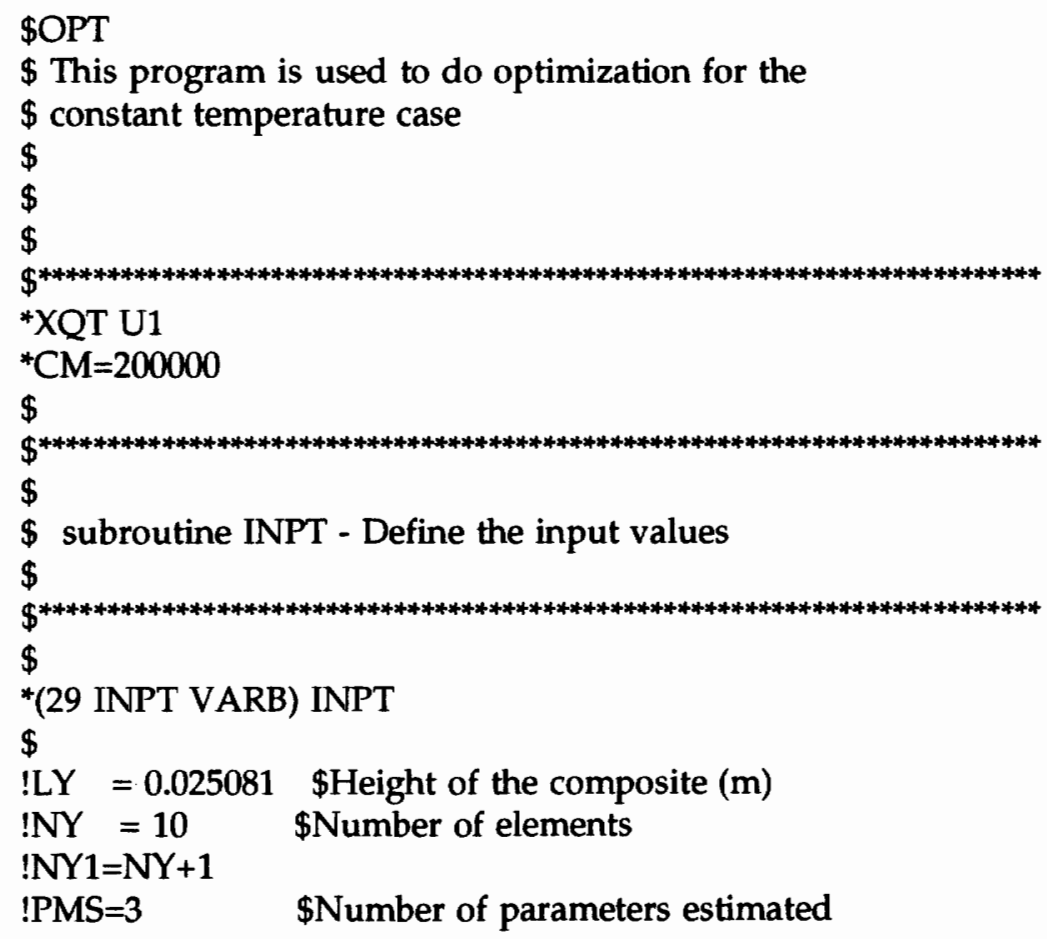




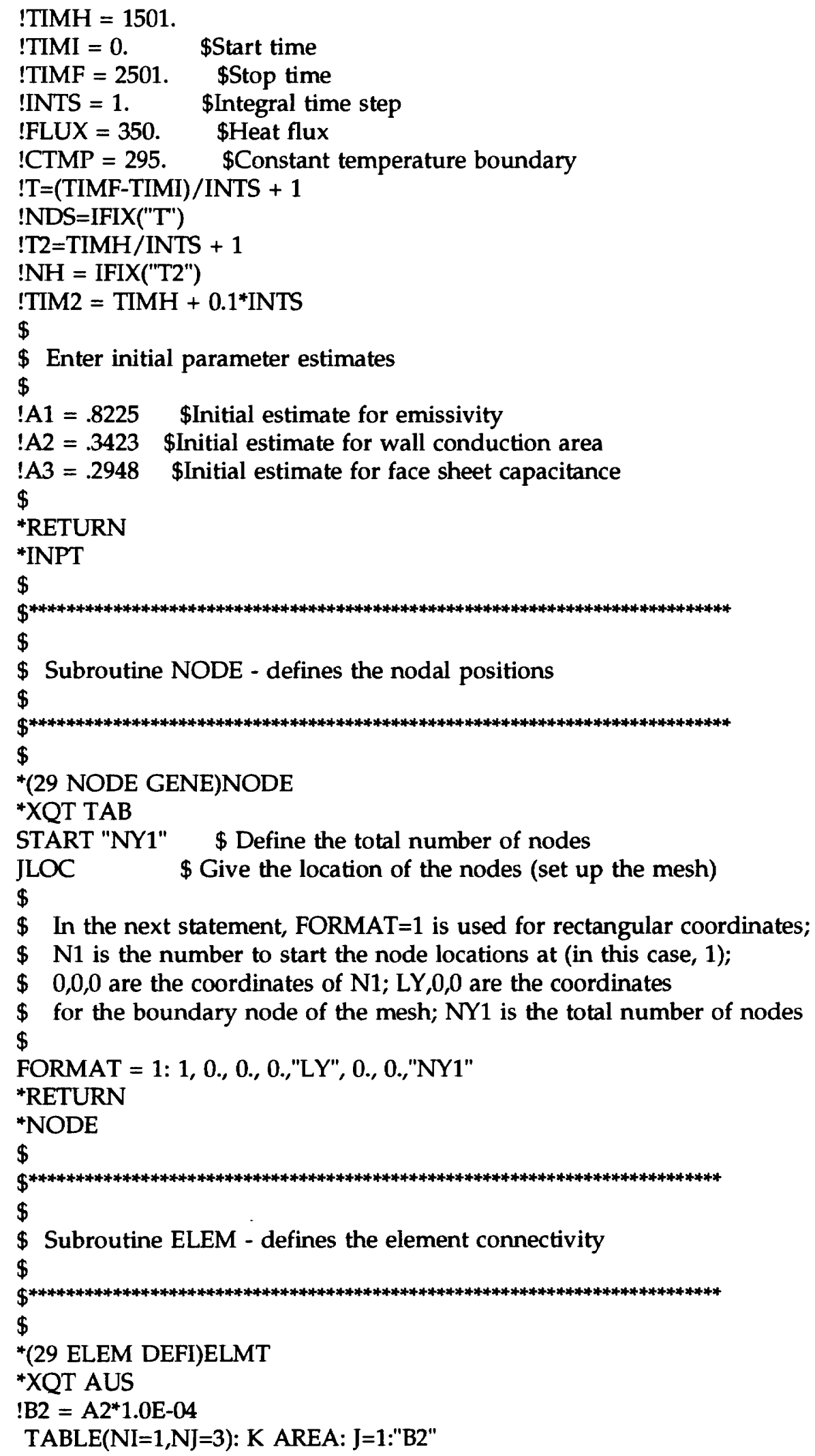


$\mathrm{J}=3: 2.14 \mathrm{E}-6$

$\$$

RESET NUTED $=1$

$\mathrm{K} 21$

GROUP 1 \$web core

121 "NY"

$\$$

$\$$

\$ The point heat source is also defined as a K21 element. Note the

$\$$ thermal conductivities and the specific heat must be set to zero in

$\$$ COND PROP 2 . In addition the two ends must have the same node number.

$\$$

GROUP 2 \$Source heat flux node

$\mathrm{NSECT}=\mathbf{2}$

NMAT $=2 \quad$ \$material 2

11

GROUP 3 \$face sheet capacitance nodes

$\mathrm{NSECT}=3$

NMAT $=3$

11 : "NY1" "NY1"

R21 \$radiation elements in core

GROUP 1

NSECT $=1$

11

GROUP 2

NSECT $=2$

12110

GROUP 3

NSECT $=1$

"NY1" "NY1"

$\$$

*XQT TGEO

*RETURN

${ }^{*}$ ELMT

$\$$

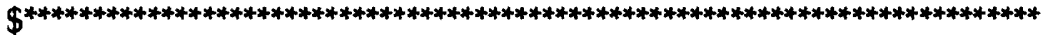

$\$$

\$ Subroutine TABL - Defines conduction properties

$\$ \quad$ Defines heat flux and constant temperature boundary

$\$$

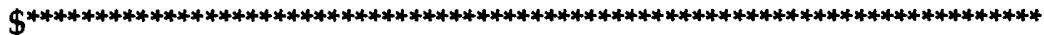

$\$$

*(29 TABL GENE)TABL

*XQT AUS

$\$$

TABLE(NI=1,NJ=1): TEMP NODE: J=1: 11 .

TABLE(NI=1,NJ=1): APPL TEMP: J=1: "CTMP"

TABLE(NI=9,NJ=2): COND PROP 1: I = 123456 .

$\mathrm{J}=1$ : 293. 4420. 558.27 .57 .57 .5 
$\mathrm{J}=2: 533.4420 .619 .510 .710 .710 .7$

TABLE(NI=9,NJ=1): COND PROP 2: $\mathrm{I}=3456$

$\mathrm{J}=1: 0.0 .0 .0$.

TABLE(NI=1,NJ=2): R CIRC: J=1: .001384

TABLE(NI=1,NJ=1): SB CONS: ${ }^{\mathrm{J}=2: .15378} \mathrm{~J}=1: 5.6697 \mathrm{E}-8$

$\$$

TABLE(NI=1,NJ=1): SOUR K21 2: BLOCK 1: J=1: "FLUX"

BLOCK 2: J=1: "FLUX"

BLOCK 3: J=1: 0.

BLOCK 4: J=1: 0 .

$\$$

TABLE(NI=1,NJ=4): SOUR TIME: $\mathrm{J}=1: 0.0$

$\mathrm{J}=2$ : "TIMH"

J=3: "TIM2"

$\mathrm{J}=4:$ "TIMF"

*RETURN

*TABL

$\$$

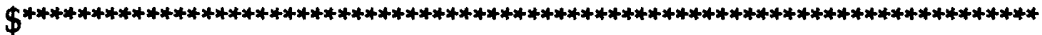

$\$$

$\$$ Subroutine UPDA - Updates thermal property values

$\$$

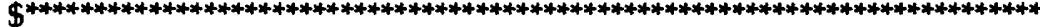

$\$$

*(29 TABL UPDA) UPDA

*XQT AUS

$\$$

!AR $=1.0-" \mathrm{~A} 1 "$

!B3 $=\mathrm{A}^{*} 1.0 \mathrm{E}+07$

TABLE(NI=9,NJ=1): COND PROP 3: $\mathrm{I}=23456$

OPER $=$ XSUM

$\mathrm{J}=1: 1$. "B3" 0.0 .0 .

TABLE(NI=4,NJ=1): RADI PROP 1: I = 234

OPERATION=XSUM

$\mathrm{J}=1$ : "A1" "A1" "AR"

$\$$

${ }^{*}$ RETURN

*UPDA

$\$$

$\$$ \$***************************************************************************

$\$$

*(29 BILD TS) XXXX

$\$$

$\$$ Bring surface temperature data from TRAN TEMP multi-block data set using

$\$$ XSUM and TRANSFER.

$\$$

$\$$ \$***************************************************************************

$\$$

$\$ ! N T N=$ N1-1 $\$$ The surface node -1 (necessary for SBASE in TRANSFER)

${ }^{*}$ XQT AUS 


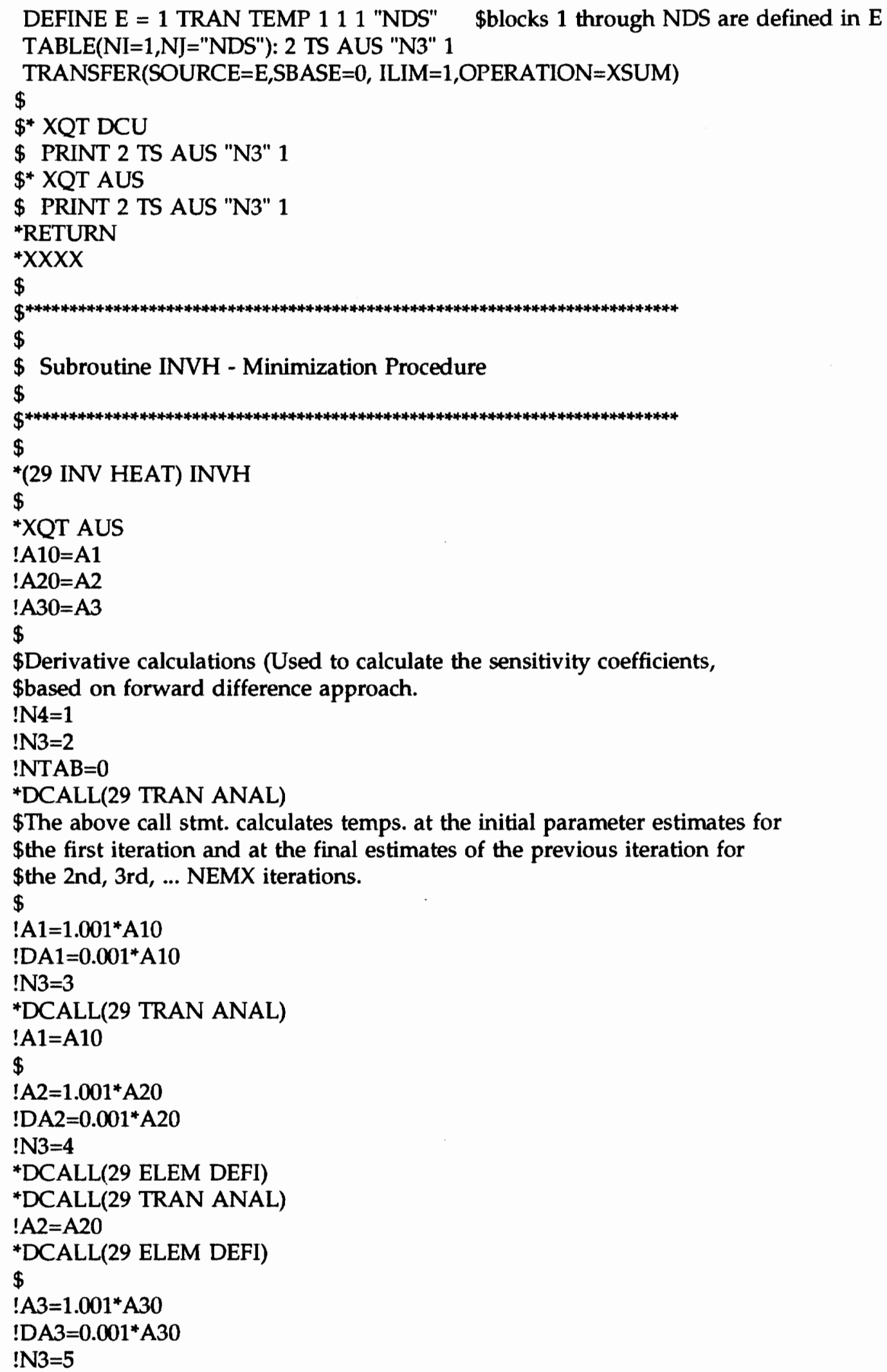




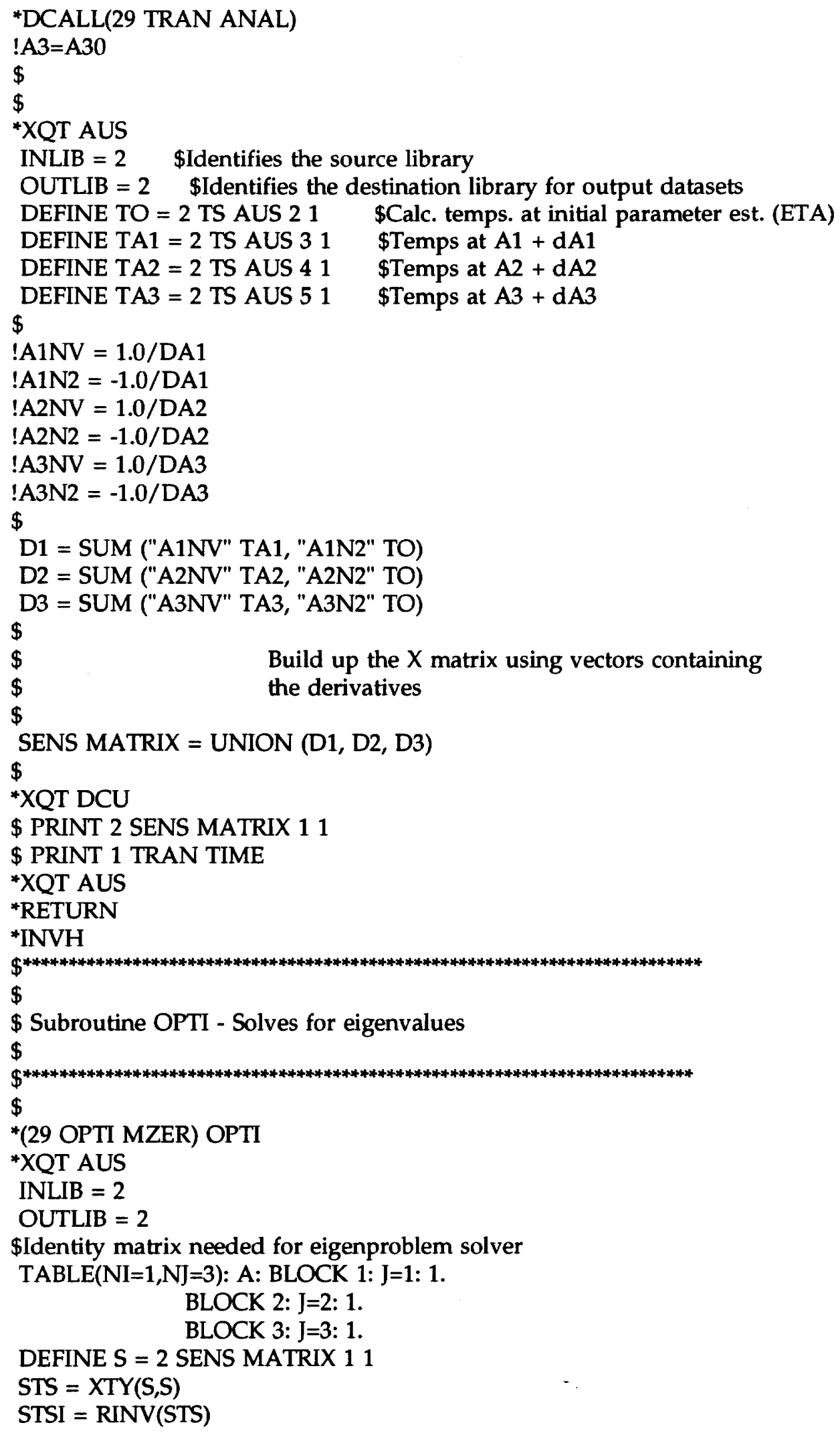




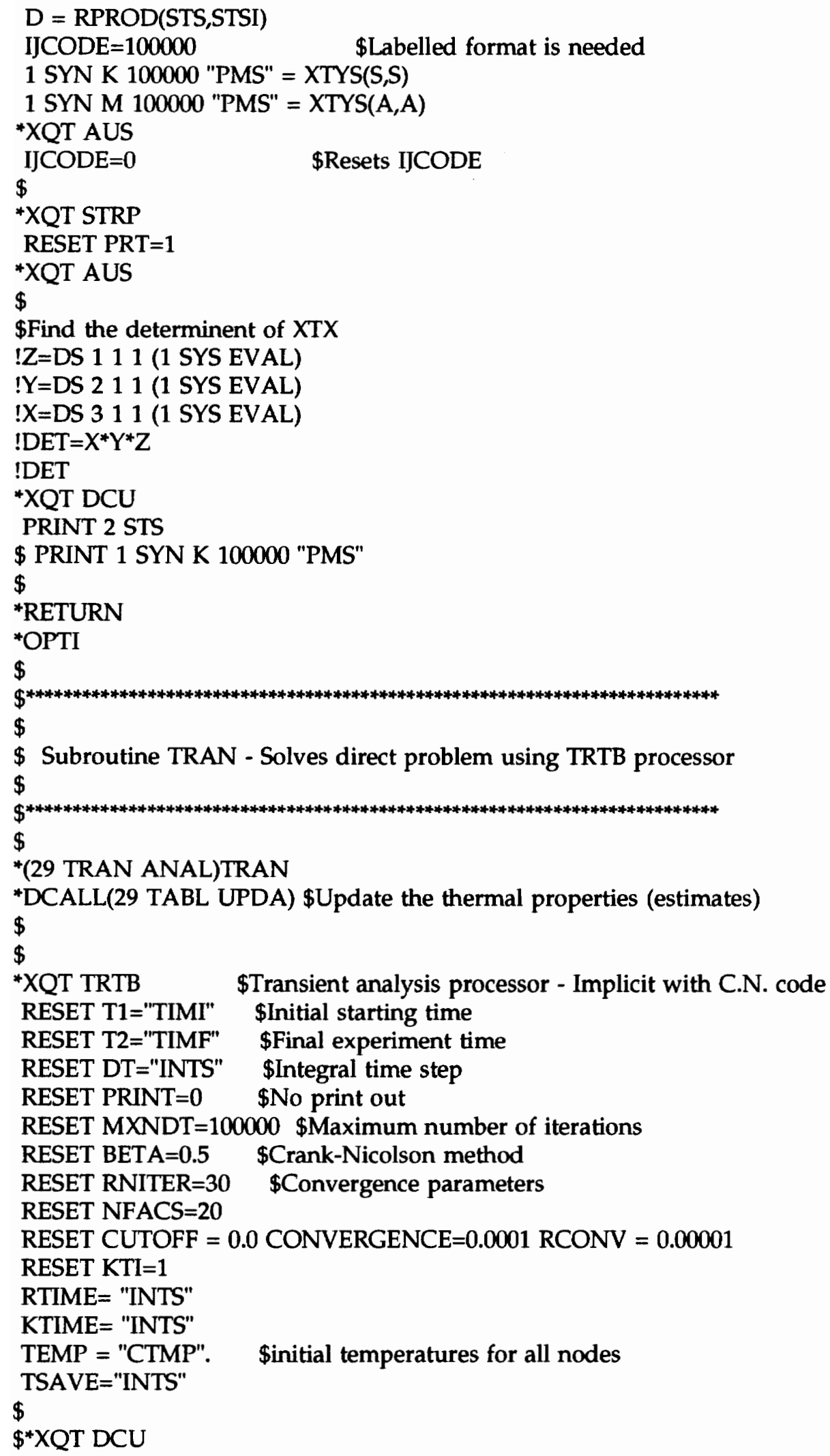




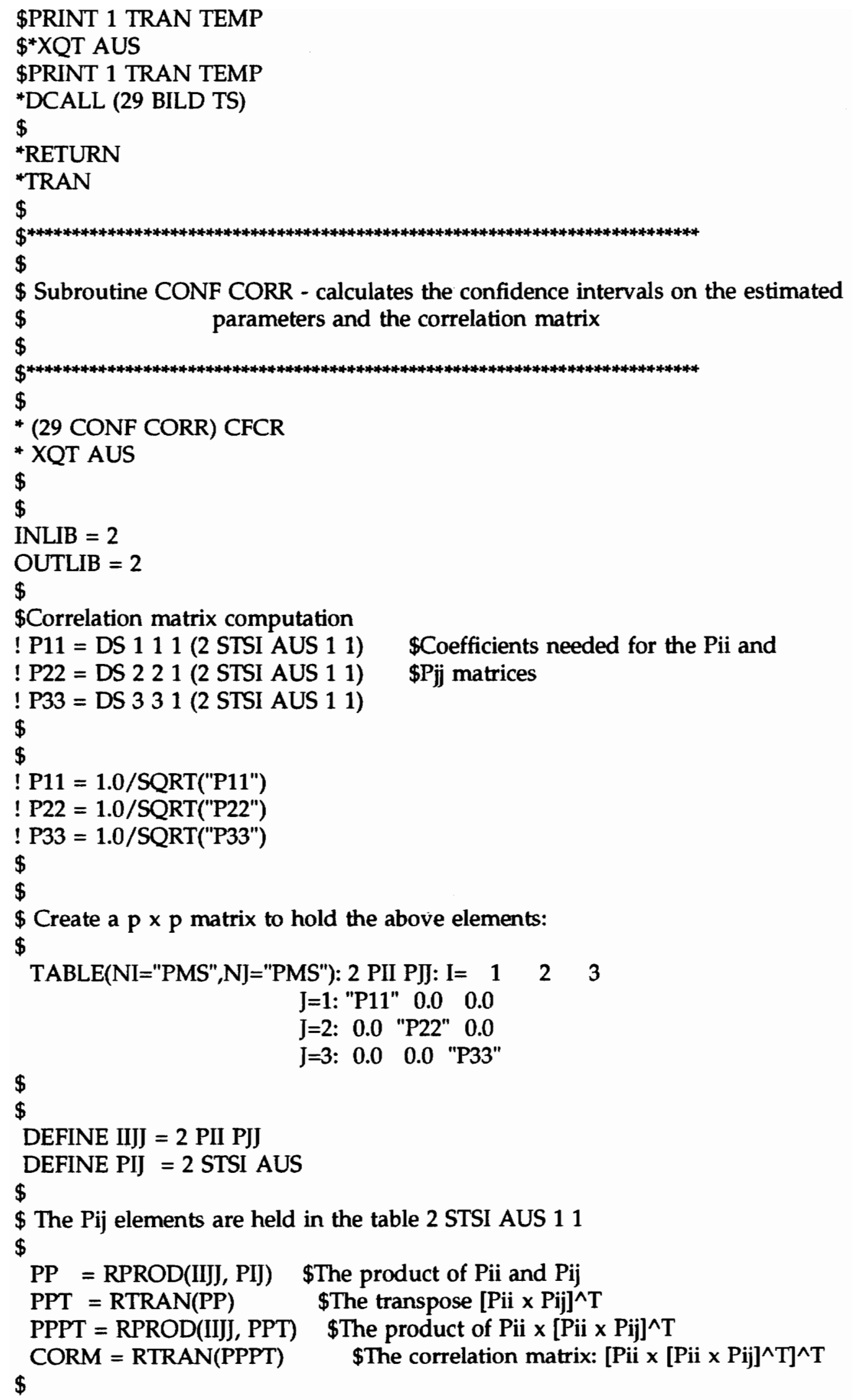




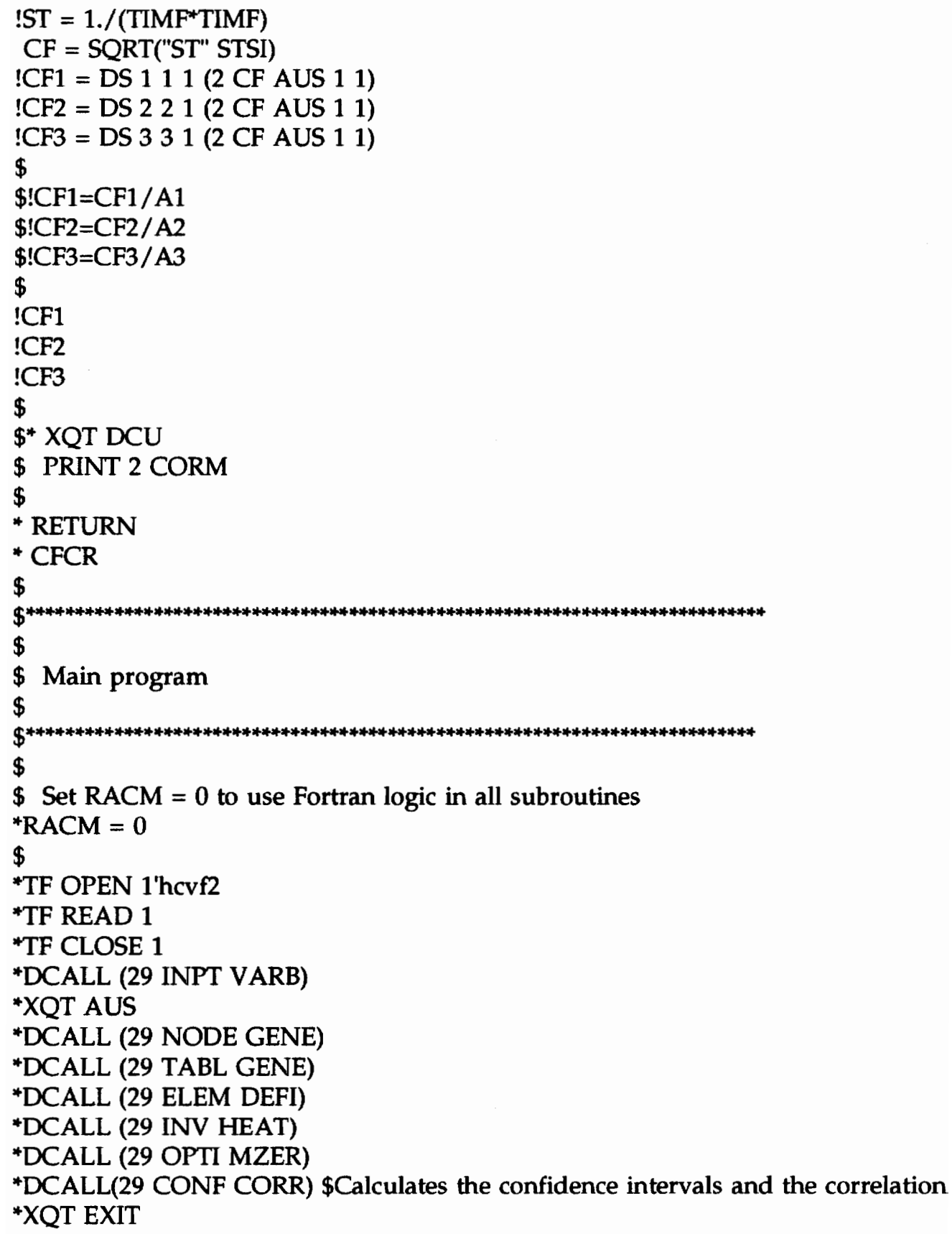




\section{APPENDIX D}

\section{Radiation View Factor Code}

This program uses a Monte Carlo method to calculate radiation view factors

within the core of the honeycomb. It is based on the theory given in Chapter 3.

Table D.1 Radiation View Factor Code

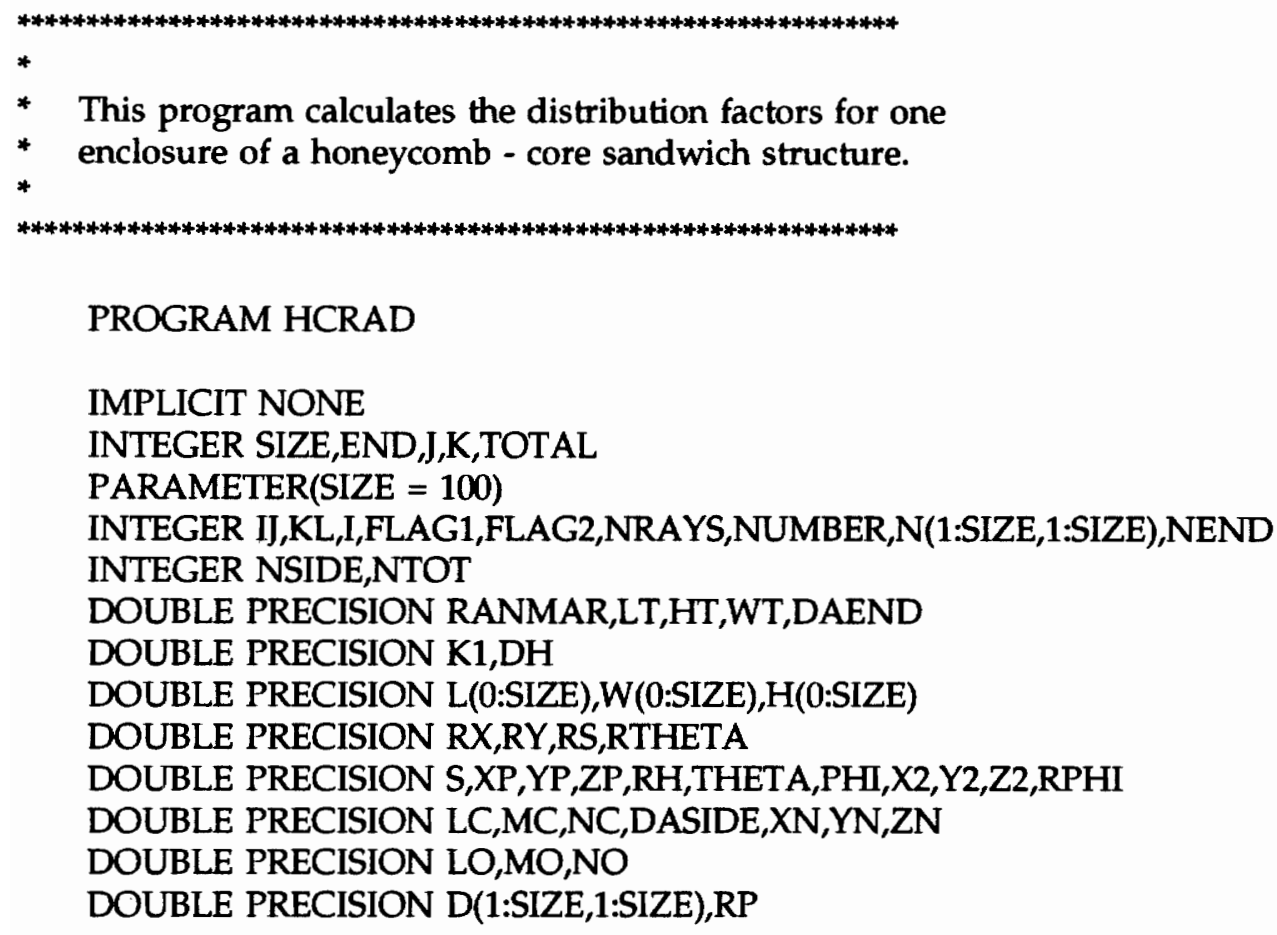

*

* This program calculates the distribution factors for one

* enclosure of a honeycomb - core sandwich structure.

$* * * * * * * * * * * * * * * * * * * * * * * * * * * * * * * * * * * * * * * * * * * * * * * * * * * * * * * * * * * * * * * * *$

PROGRAM HCRAD

IMPLICIT NONE

INTEGER SIZE,END,J,K,TOTAL

PARAMETER(SIZE $=100$ )

INTEGER IJ,KL,I,FLAG1,FLAG2,NRAYS,NUMBER,N(1:SIZE,1:SIZE),NEND

INTEGER NSIDE,NTOT

DOUBLE PRECISION RANMAR,LT,HT,WT,DAEND

DOUBLE PRECISION K1,DH

DOUBLE PRECISION L(0:SIZE),W(0:SIZE),H(0:SIZE)

DOUBLE PRECISION RX,RY,RS,RTHETA

DOUBLE PRECISION S,XP,YP,ZP,RH,THETA,PHI,X2,Y2,Z2,RPHI

DOUBLE PRECISION LC,MC,NC,DASIDE,XN,YN,ZN

DOUBLE PRECISION LO,MO,NO

DOUBLE PRECISION D(1:SIZE,1:SIZE),RP 
******Initialize random number generator

IJ $=12345$

$\mathrm{KL}=23456$

******Enclosure dimensions

$\mathrm{LT}=0.043315 \mathrm{D} 0$

$\mathrm{HT}=0.025081 \mathrm{D} 0$

$\mathrm{WT}=0.032074 \mathrm{D} 0$

******Total number of elements and rays

NTOT $=12$

NUMBER $=10000000$

$\operatorname{OPEN}(2$, FILE $=$ 'DIST.DAT')

CALL RMARIN(IJ,KL)

****** Define element areas

$\mathrm{K} 1=\mathrm{LT} / \mathrm{WT}$

DAEND $=\mathrm{LT}^{*} \mathrm{WT}$

NEND $=1$

$\mathrm{NSIDE}=10$

DASIDE $=2 . \mathrm{D}^{*} \mathrm{HT}^{*}(\mathrm{LT}+\mathrm{WT}) / \mathrm{DBLE}(\mathrm{NSIDE})$

$\mathrm{DH}=\mathrm{HT} / \mathrm{DBLE}$ (NSIDE)

NTOT $=12$

DO $I=1$,NTOT

$W(I)=0 . D 0$

$\mathrm{L}(\mathrm{I})=0 . \mathrm{D} 0$

$\mathrm{H}(\mathrm{I})=0 . \mathrm{DO}$

END DO

DO $I=1$, NTOT

DO $\mathrm{J}=1$,NTOT

$\mathrm{N}(\mathrm{I}, \mathrm{J})=0 . \mathrm{D} 0$

END DO

END DO

******Define geometry of elements

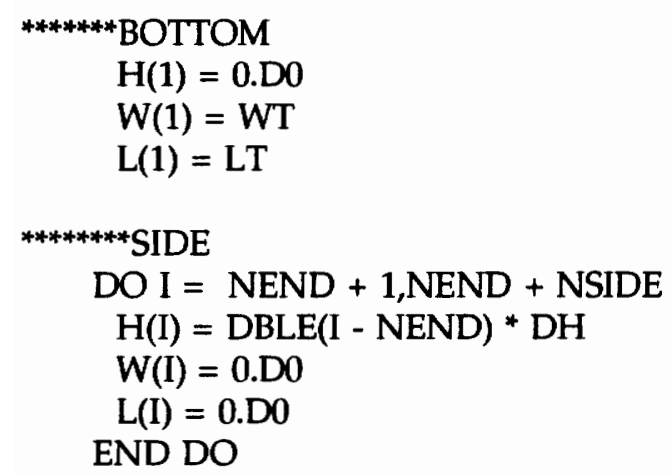




$$
\begin{aligned}
& \text { W(12) }=\mathrm{WT} \\
& \mathrm{L}(12)=\mathrm{LT} \\
& \mathrm{H}(12)=\mathrm{HT}
\end{aligned}
$$

*******Begin ray trace at a random location

$$
\begin{aligned}
& \text { END }=6 \\
& \text { DO I }=1, \text { END } \\
& \text { IF (I .EQ. 1) THEN } \\
& \text { NRAYS = NUMBER } \\
& \text { ELSE } \\
& \text { NRAYS = INT(DBLE(NUMBER)*DASIDE/DAEND) } \\
& \text { END IF } \\
& \text { DO J = 1,NRAYS } \\
& \text { IF (I .EQ. 1) THEN } \\
& \text { RX = RANMAR() } \\
& \text { RY = RANMAR() } \\
& \text { XP }=(\text { RX }-0.5 D 0)^{*} \text { WT } \\
& \text { YP }=(\text { RY }-0.5 D 0)^{*} \text { LT } \\
& \text { ZP }=0 . D 0 \\
& \text { FLAG } 1=1
\end{aligned}
$$

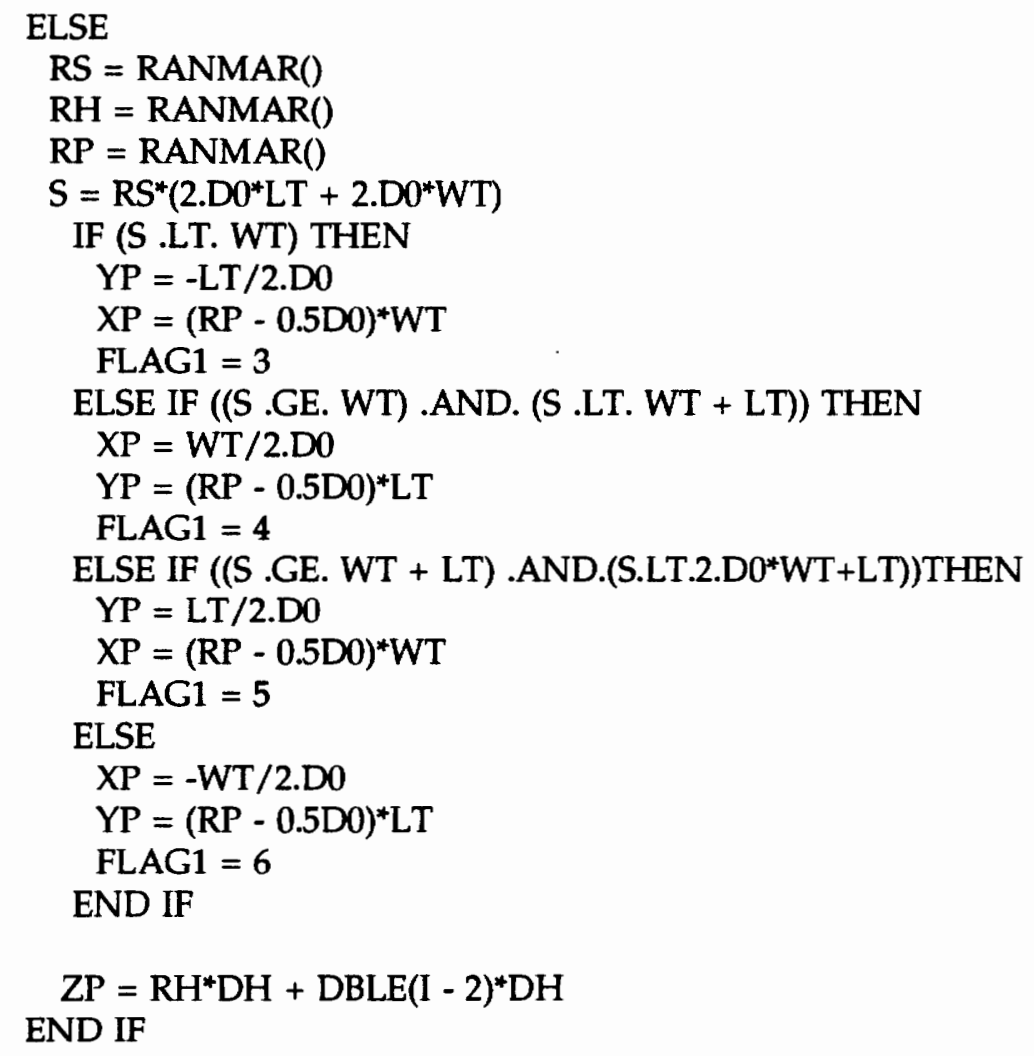




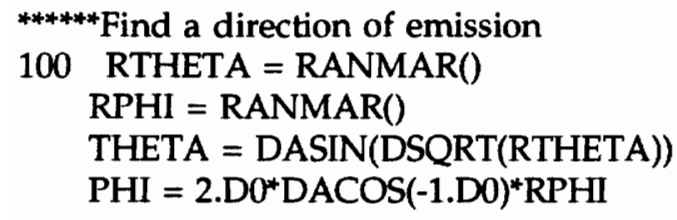

***** Define local and global direction cosines

$$
\begin{aligned}
& \text { LO = DSIN(THETA) }{ }^{*} \text { DCOS(PHI) } \\
& \text { MO = DSIN(THETA) } * \text { DSIN(PHI) } \\
& \text { NO = DCOS(THETA) } \\
& \text { IF (DABS(LO) .LT. 1.D-7) THEN }
\end{aligned}
$$
LO = 1.D-7

END IF

IF (DABS(MO) .LT. 1.D-7) THEN $\mathrm{MO}=1 . \mathrm{D}-7$

END IF

IF (DABS(NO) .LT. 1.D-7) THEN $\mathrm{NO}=1 . \mathrm{D}-7$

END IF

IF (FLAG1 .EQ. 1) THEN

$$
\begin{aligned}
& \mathrm{LC}=\mathrm{LO} \\
& \mathrm{MC}=\mathrm{MO} \\
& \mathrm{NC}=\mathrm{NO}
\end{aligned}
$$

ELSE IF (FLAG1 .EQ. 2) THEN

$$
\begin{aligned}
& \mathrm{LC}=\mathrm{LO} \\
& \mathrm{MC}=\mathrm{MO} \\
& \mathrm{NC}=-\mathrm{NO}
\end{aligned}
$$

ELSE IF (FLAG1 .EQ. 3) THEN

$$
\begin{aligned}
& \mathrm{LC}=\mathrm{LO} \\
& \mathrm{MC}=\mathrm{NO} \\
& \mathrm{NC}=-\mathrm{MO}
\end{aligned}
$$

ELSE IF (FLAG1 .EQ. 4) THEN

$$
\mathrm{LC}=-\mathrm{NO}
$$$$
\mathrm{MC}=\mathrm{MO}
$$$$
\mathrm{NC}=\mathrm{LO}
$$

ELSE IF (FLAG1 .EQ. 5) THEN

$$
\begin{aligned}
& \text { LC }=\text { LO } \\
& M C=-N O \\
& N C=M O \\
& \text { ELSE } \\
& \text { LC }=\text { NO } \\
& M C=M O \\
& \text { NC }=- \text { LO } \\
& \text { END IF }
\end{aligned}
$$

******Find where the ray intercepts the enclosure

$$
\mathrm{Z} 2=0 . \mathrm{D} 0
$$




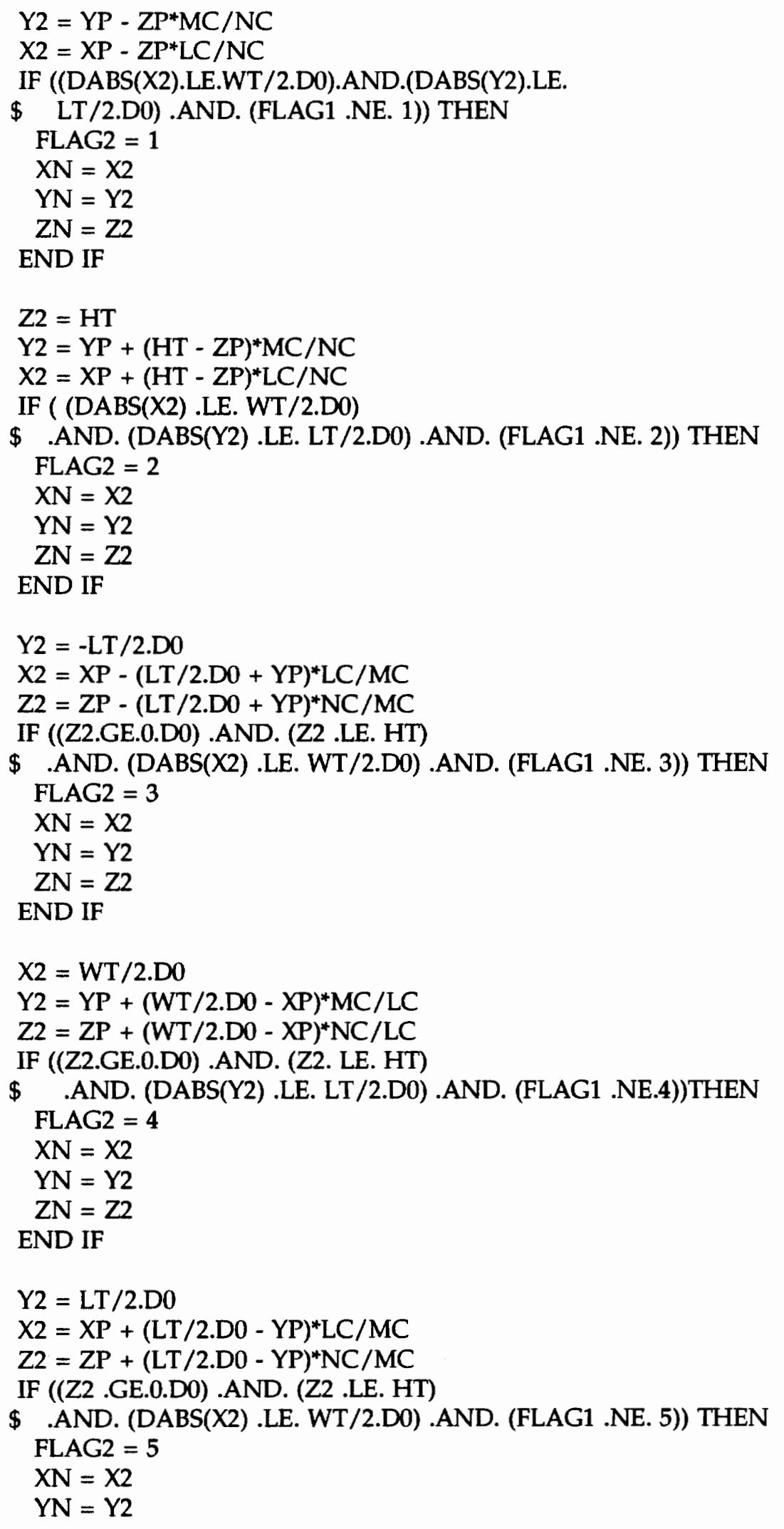


$\mathrm{ZN}=\mathrm{Z2}$

END IF

$\mathrm{X} 2=-\mathrm{WT} / 2 . \mathrm{D} 0$

$\mathrm{Y} 2=\mathrm{YP}-(\mathrm{WT} / 2 . \mathrm{D} 0+\mathrm{XP})^{*} \mathrm{MC} / \mathrm{LC}$

$\mathrm{Z} 2=\mathrm{ZP}-(\mathrm{WT} / 2 . \mathrm{D} 0+\mathrm{XP})^{*} \mathrm{NC} / \mathrm{LC}$

IF ((Z2.GE.0.D0) .AND. (Z2 .LE. HT)

$\$$.AND. (DABS(Y2) .LE. LT/2.D0) .AND. (FLAG1 .NE. 6))THEN

FLAG2 $=6$

$X N=X 2$

$\mathrm{YN}=\mathrm{Y} 2$

$\mathrm{ZN}=\mathrm{Z2}$

END IF

******Determine which element the ray hits

IF (FLAG2 .EQ. 1) THEN

$\mathrm{K}=1$

GOTO 125

ELSE IF (FLAG2 .EQ. 2) THEN

$K=12$

GOTO 125

ELSE

$\mathrm{K}=2+\mathrm{INT}(\mathrm{ZN} / \mathrm{DH})$

END IF

$125 \mathrm{~N}(\mathrm{I}, \mathrm{K})=\mathrm{N}(\mathrm{I}, \mathrm{K})+1$

END DO

END DO

******inish filling $\mathrm{N}$ matrix

DO $I=1,6$

$\mathrm{DO} \mathrm{K}=1,12$

$\mathrm{N}(13-\mathrm{I}, 13-\mathrm{K})=\mathrm{N}(\mathrm{I}, \mathrm{K})$

END DO

END DO

****** Use $\mathrm{N}$ matrix to calculate D matrix

$\mathrm{I}=1$

DO $K=1$, NTOT

$\mathrm{D}(\mathrm{I}, \mathrm{K})=\mathrm{N}(\mathrm{I}, \mathrm{K}) / \mathrm{DBLE}(\mathrm{NUMBER})$ 
END DO

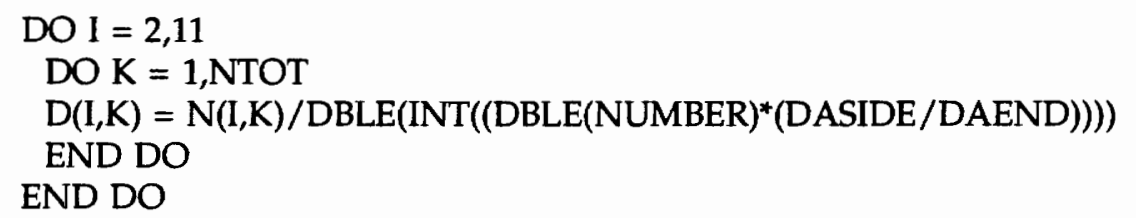

$$
\begin{aligned}
& I=12=1, N T O T \\
& D O ~ K=1, N(I, K) / D B L E(N U M B E R) \\
& D(I, K)=N(I, K) \\
& \text { END DO }
\end{aligned}
$$

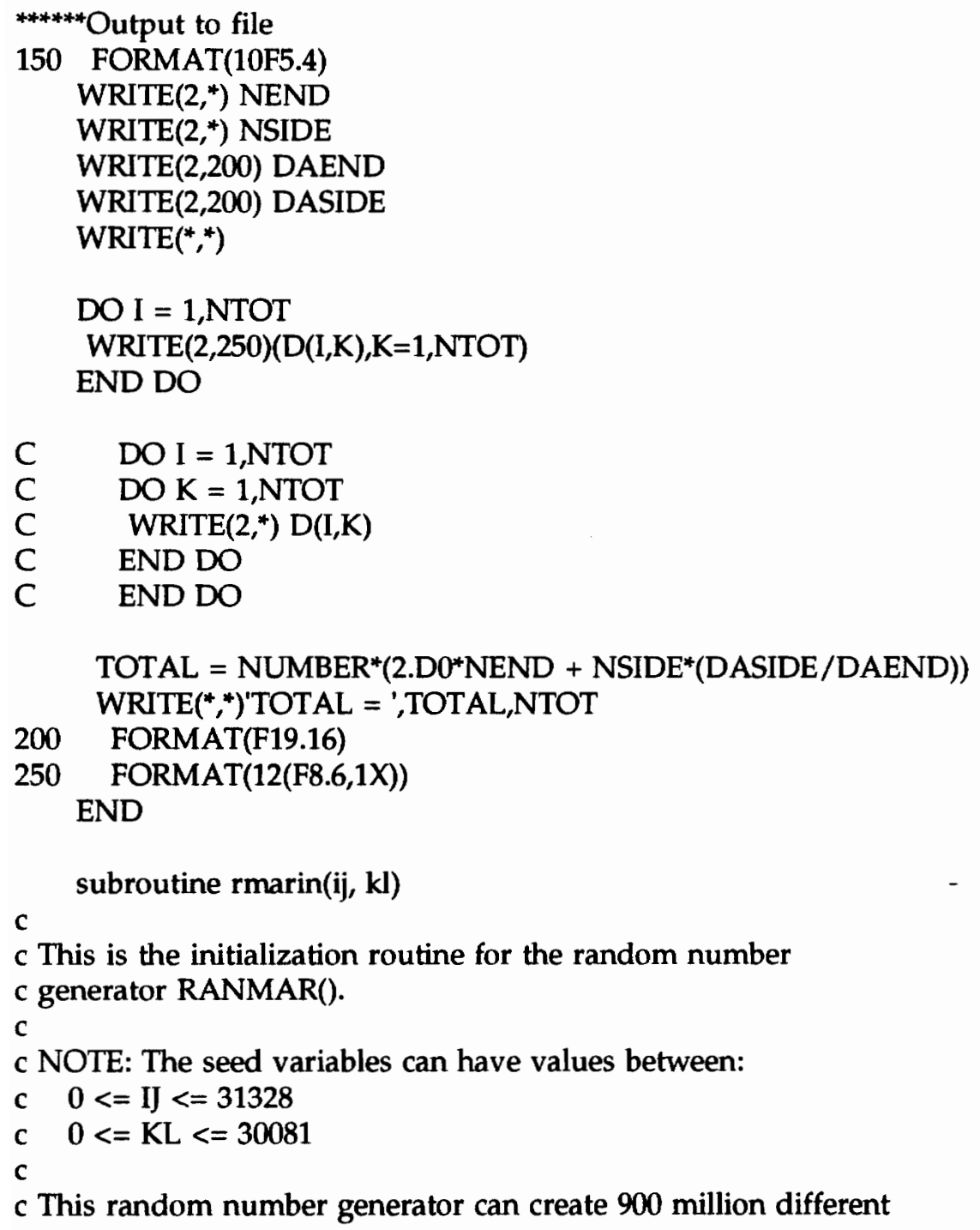


c subsequences -- with each subsequence having a length of c approximately $10^{\wedge} 30$.

c

c Use IJ $=1802 \& \mathrm{KL}=9373$ to test the random number generator.

c The subroutine RANMAR should be used to generate 20000 random

$c$ numbers. Then display the next six random numbers generated

c multiplied by $4096^{*} 4096$. If the random number generator is

c working properly, the random numbers should be:

c

c

C

real ${ }^{*} 8 \mathrm{u}(97), \mathrm{c}, \mathrm{cd}, \mathrm{cm}, \mathrm{s}, \mathrm{t}$

integer $\mathrm{ii}, \mathrm{i}, \mathrm{j}, \mathrm{ij}, \mathrm{jj}, \mathrm{k}, \mathrm{kl}, \mathrm{l}, \mathrm{m}, \mathrm{i97}, \mathrm{j} 97$

logical test

common / raset1/ u, c, cd, cm, i97, j97, test

test $=$.false.

C

if( IJ .lt. 0 .or. IJ .gt. 31328 .or.

1 KL .lt. 0 .or. KL .gt. 30081 ) then

write $\left({ }^{*},{ }^{*}\right)$ ' The first random number seed must have a'

write $(*, *)$ ' value between 0 and 31328.'

write $(*, *)$

write $(*, *)$ 'The second seed must have a value between'

write $(*, *)$ ' and 30081 .'

write $\left({ }^{*},{ }^{*}\right)$ 'Stopping...'

stop

endif

c

$\mathrm{i}=\bmod (\mathrm{IJ} / 177,177)+2$

$\mathrm{j}=\bmod (\mathrm{IJ}, 177)+2$

$k=\bmod (K L / 169,178)+1$

$1=\bmod (k l, \quad 169)$

c

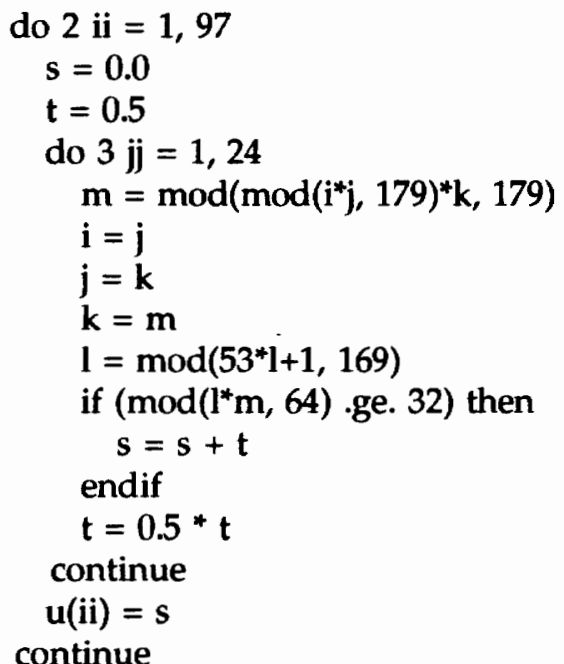




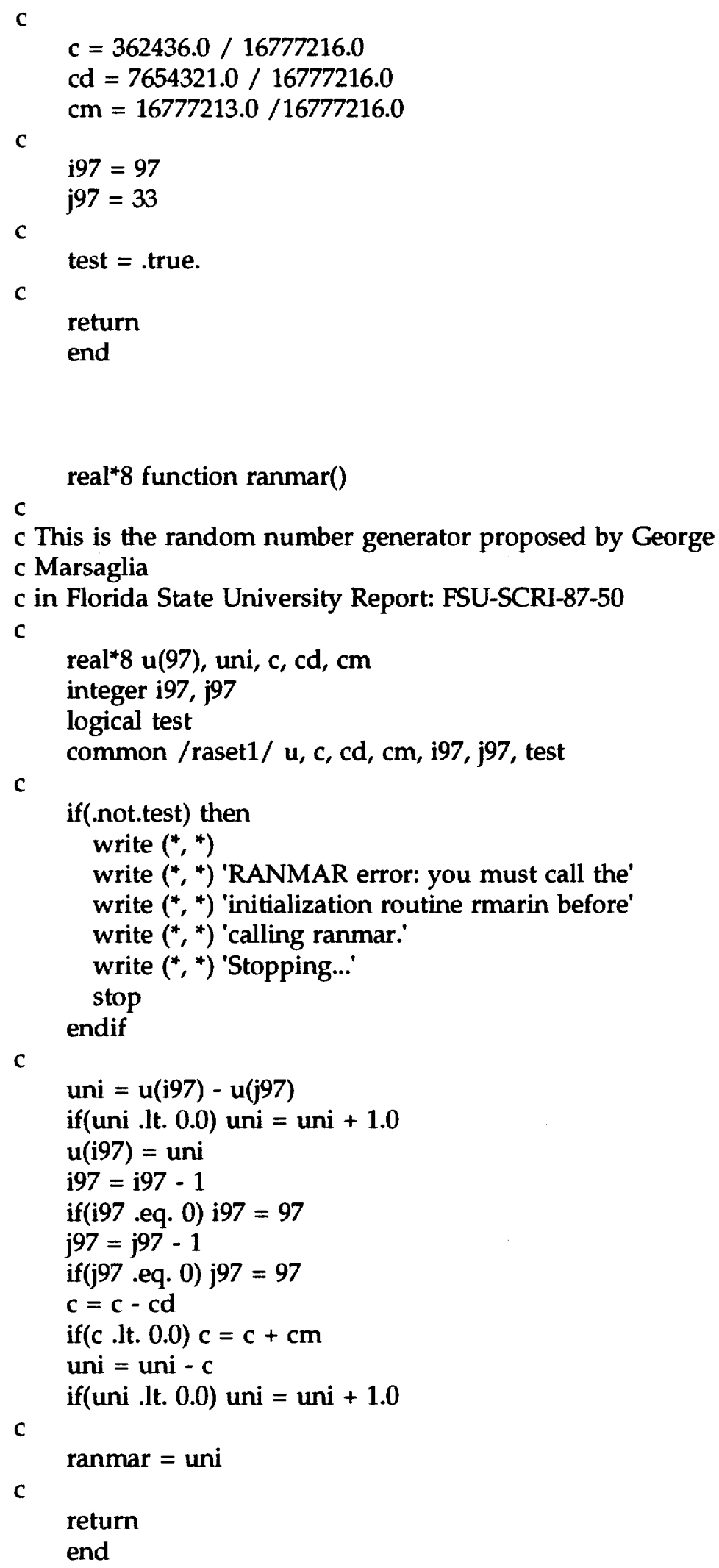

end 


\section{Vita}

The author, David C. Copenhaver, was born on March 2, 1972. He was raised by his parents, Clyde and Jean Copenhaver, in Crockett, Virginia. He graduated from Rural Retreat High School in June, 1990. His formal study of engineering began in August, 1990 at Virginia Polytechnic Institute \& State University. He received the Bachelor of Science degree in Mechanical Engineering from Virginia Tech in May, 1994. That fall he enrolled in graduate school at Virginia Tech, under the direction of Dr. Elaine P. Scott. With the completion of this thesis he has fulfilled the requirements for the Master of Science Degree in Mechanical Engineering on this the 21st day of March, 1996. While he eventually plans to pursue a $\mathrm{PhD}$ degree he has decided to begin a career in industry with Framatome Nuclear Fuels in Lynchburg, Virginia.

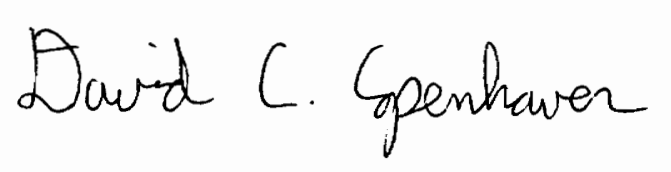

